\title{
Blow-up de soluções positivas de equações semilineares
}

\author{
Fernanda Tomé Alves
}

Orientador: Prof. Dr. Alexandre Nolasco de Carvalho

Dissertação apresentada ao Instituto de Ciências Matemáticas e de Computação da Universidade de São Paulo, como parte dos requisitos para obtenção do título de Mestre em Ciências na área: Matemática.

USP - São Carlos

Março/2006 
Aos meus pais e ao meu marido, com muito amor... 


\section{Agradecimentos}

Com esforço e dedicação mais uma etapa foi vencida em minha vida. Com orgulho e com sentimento de realização, apresento aqui o resultado de muito estudo, trabalho e horas de questionamentos e soluções. Porém, nada disso teria sido possível se não tivesse ao meu lado pessoas admiráveis, amadas, fortes e amigas para me ajudar, orientar, encorajar e me fazer acreditar que era possível. É com muita admiração e respeito que agradeço, primeiramente, meu orientador Prof. Alexandre e o Prof. Sérgio. Obrigada pela confiança, pela ajuda nas horas em que se acredita que não haja mais solução e por alimentarem em mim um amor cada vez maior pela matemática. Aos professores do IBILCE-UNESP, grandes incentivadores, registro aqui meus sinceros agradecimentos. Um agradecimento especial à FAPESP que acreditou no meu trabalho e na minha capacidade como pesquisadora. Agradeço meus colegas de curso, docentes e funcionários do ICMC.

Agradeço ainda a pessoa mais especial durante toda essa batalha: meu marido. Obrigada Cláudio pelo amor imensurável, pelo companheirismo, por todo o incentivo e por ter feito eu acreditar em mim mesma. Agradeço meus pais e irmãs pelo grande amor, pelo ombro nas horas de desespero e pela felicidade nas horas de vitória. Não poderia deixar de agradecer também meus amigos por toda a força que me deram. Agradeço o pessoal da salinha 3-023 e, em especial, Amanda, Gabriel, Judith e Michelle, pela amizade que foi construída e cultivada durante esses dois anos, que trouxe momentos de muita alegria, risadas e aprendizado. Obrigada ainda Karina, Ricardo e Marcelinho pela dedicação ao trabalho e esforço conjuntos.

É difícil dizer em palavras todo o carinho e gratificação por todos que, diretamente ou não, me ajudaram a trilhar esse caminho tão importante em minha vida. Que cada um saiba que de alguma maneira foram responsáveis por essa conquista. Muito obrigada! 



\section{Abstract}

Consider the initial-boundary value problem

$$
\begin{aligned}
u_{t}=\Delta u+f(u) \quad & \text { in } \quad \Omega \times(0, T), \\
u(x, 0)=\phi(x) & \text { if } \quad x \in \Omega, \\
u(x, t)=0 & \text { if } \quad x \in \partial \Omega, 0<t<T,
\end{aligned}
$$

where $\Omega$ is a bounded domain in $R^{n}$ with $C^{2}$ boundary, $f$ is continuously differentiable with $f(s)>0$, and $\phi$ is nonnegative and smooth on $\bar{\Omega}$ with $\phi=0$ on $\partial \Omega$. Assume that the unique solution $u(x, t)$ blows up in finite time $T<\infty$. The question addressed is: where does the blow-up occur? In this work we prove: if $\Omega=B_{R} \subset R^{n}$, then blow-up occurs only at $r=0$. Moreover, if $f(u)=u^{p}, p>1$, then $u(r, t) \leq C / r^{2}(\gamma-1)$ for any $1<\gamma<p$, and hence $\lim \sup _{t \rightarrow T^{-}}\|u(\cdot, t)\|_{q}<\infty$ if $q<n(p-1) / 2$. In the nonsymmetric case where $\Omega$ is a convex domain, we prove that the blow-up set lies in a compact subset of $\Omega$. If $f(u)=u^{p}, p>1$, then $u(x, t) \leq C /(T-t)^{1 / p-1}$ and, if $n=1,2$, or if $n \geq 3$ and $p \leq(n+2) /(n-2)$, then $\tau^{\beta} u(x+\xi, T-\tau) \rightarrow c_{0}$ as $\tau \rightarrow 0^{+}$if $|\xi| \leq C \tau^{1 / 2}$ and $c_{0}=0$ or $\beta^{\beta}$ where $\beta=(p-1)^{-1}$. Elementary applications of the Maximum Principle are used to prove the essential estimate for the proofs of these results. 



\section{Resumo}

Considere o problema de valor inicial e de fronteira

$$
\begin{array}{rll}
u_{t}=\Delta u+f(u) & \text { em } & \Omega \times(0, T), \\
u(x, 0)=\phi(x) & \text { se } & x \in \Omega, \\
u(x, t)=0 & \text { se } & x \in \partial \Omega, 0<t<T,
\end{array}
$$

onde $\Omega$ é um domínio limitado em $\mathbb{R}^{n}$ com bordo $C^{2}, f$ é continuamente diferenciável com $f(s)>0$, e $\phi$ é não-negativa e suave sobre $\bar{\Omega} \operatorname{com} \phi=0$ sobre $\partial \Omega$. Suponha que a única solução $u(x, t)$ possui blow-up em tempo finito $T<\infty$. A questão que se coloca é: onde ocorre o blow-up? Neste trabalho provamos que: se $\Omega=B_{R} \subset R^{n}$, então o blow-up ocorre apenas em $r=0$. Além disso, se $f(u)=u^{p}, p>1$, então $u(r, t) \leq C / r^{2}(\gamma-1)$ para qualquer $1<\gamma<p$, e assim $\lim \sup _{t \rightarrow T^{-}}\|u(\cdot, t)\|_{q}<\infty$ se $q<n(p-1) / 2$. No caso não-simétrico onde $\Omega$ é um domínio convexo, provamos que o conjunto de blow-up é um subconjunto compacto de $\Omega$. Se $f(u)=u^{p}, p>1$, então $u(x, t) \leq C /(T-t)^{1 / p-1}$ e, se $n=1,2$, ou se $n \geq 3$ e $p \leq(n+2) /(n-2)$, então $\tau^{\beta} u(x+\xi, T-\tau) \rightarrow c_{0}$ quando $\tau \rightarrow 0^{+}$se $|\xi| \leq C \tau^{1 / 2}$ e $c_{0}=0$ ou $\beta^{\beta}$ onde $\beta=(p-1)^{-1}$. As provas das estimativas essenciais para a demonstração desses resultados são feitas utilizando o Princípio do Máximo. 



\section{Sumário}

\begin{tabular}{|l|l}
\hline Agradecimentos & i
\end{tabular}

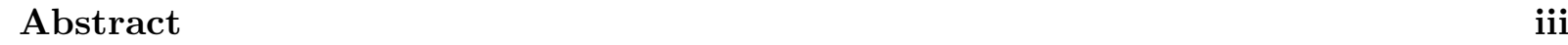

Resumo $\quad$ v

Introdução 1

1 Preliminares 5

$1.1 \quad$ Semigrupos e seus geradores $\ldots \ldots \ldots \ldots \ldots \ldots$

$1.2 \quad$ O Teorema de Hille-Yosida $\ldots \ldots \ldots \ldots \ldots \ldots$

$1.3 \quad$ O Teorema de Lumer-Phillips $\ldots \ldots \ldots \ldots$. . . . . . . . . . . . . . 12

1.4 Fórmulas exponenciais $\ldots \ldots \ldots \ldots \ldots \ldots \ldots \ldots$

1.5 Pseudo-resolventes $\ldots \ldots \ldots \ldots \ldots$

$1.6 \quad \mathrm{O}$ semigrupo dual e o Teorema de Stone . . . . . . . . . . . . . . . . . . . 22

1.7 Transformada inversa de Laplace . . . . . . . . . . . . . . . . . . . . . 27

1.8 Operadores setoriais e analiticidade . . . . . . . . . . . . . . . . . . . . 30

2 Potências fracionárias 39

2.1 Introdução . . . . . . . . . . . . . . . . . . . . . . . . . . . . 39

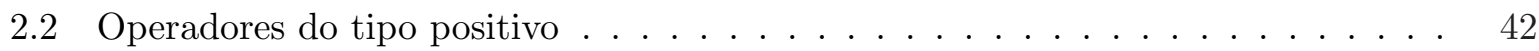

2.3 Desigualdades de interpolação para potências fracionárias . . . . . . . . . . . . 53

2.4 Potências fracionárias e semigrupos $\ldots \ldots \ldots \ldots \ldots \ldots$. . . . . . . . 57

$\begin{array}{lll}3 & \text { O problema de Cauchy } & 61\end{array}$

$3.1 \quad \mathrm{O}$ problema de Cauchy não-homogêneo . . . . . . . . . . . . . . . . . . . . 61

3.1.1 Existência, unicidade e regularidade . . . . . . . . . . . . . . . . 61

$3.2 \quad \mathrm{O}$ problema de Cauchy semilinear - o caso parabólico . . . . . . . . . . . . . . . 64 
$\begin{array}{lll}4 & \text { Blow-up } & 77\end{array}$

4.1 Introdução . . . . . . . . . . . . . . . . . . . . . . . . 77

$4.2 \quad \mathrm{O}$ caso simétrico $\ldots \ldots \ldots \ldots \ldots \ldots \ldots \ldots$

$4.3 \quad \mathrm{O}$ caso não-simétrico $\ldots \ldots \ldots \ldots$. . . . . . . . . . . . . . . . . . . 90

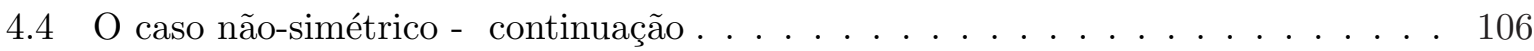

$\begin{array}{ll}\text { A O espectro do Laplaciano } & 115\end{array}$

A.1 O operador solução . . . . . . . . . . . . . . . . . . . . . . . . 115

A.2 Propriedades do operador solução . . . . . . . . . . . . . . . . . . . 116

B O Princípio do Máximo 123

B.1 Princípio do Máximo . . . . . . . . . . . . . . . . . . . . . . . . . . . . . . . 123

B.2 Princípio do Mínimo - outra versão f . . . . . . . . . . . . . . . . . . . . 126

Referências bibliográficas $\quad 129$

$\begin{array}{ll}\text { Índice remissivo } & 132\end{array}$ 


\section{Introdução}

Problemas parabólicos do tipo

$$
\left\{\begin{array}{l}
u_{t}-\Delta u=f(x, t, u, \nabla u) \text { em } D \times \mathbb{R}^{N} \\
u(x, t)=0 \text { sobre } \partial D \times \mathbb{R}^{N} \\
u(x, 0)=u_{0}(x) \geq 0
\end{array}\right.
$$

modelam um grande número de problemas físicos. A interpretação mais comum é considerar $u$ como a temperatura de uma substância em um recipiente $D \subset \mathbb{R}^{N}$ sujeita a reações químicas. Um termo de reação positivo $f$ representa uma fonte de calor devida uma reação exotérmica, isto é, uma reação química que se efetua com desprendimento de calor. Caso contrário, a reação é endotérmica. Se $f$ depende também do gradiente de $u$, então os efeitos de convecção são levados em consideração.

Diversas teorias de existência local de soluções podem ser consideradas. Soluções clássicas podem ser estabelecidas no contexto de funções Hölder contínua. Se $D$ é um domínio limitado com bordo regular e se $f$ é localmente Lipschitziana e definida para todos $u$ e $\nabla u$, então para qualquer $u_{0} \in C^{\alpha}(D), u_{0}=0$ sobre $\partial D$, existe uma solução clássica para todo $t$ suficientemente pequeno, digamos $t<T$. Quando $T=\infty$, a solução é chamada global. Via estimativas de Schauder, a solução deixa de existir se $\lim _{t \rightarrow T^{-}} \sup _{x \in \bar{D}}|u(x, t)|=\infty$ ou $\lim _{t \rightarrow T^{-}} \sup _{x \in \bar{D}}|\nabla u(x, t)|=\infty$. Neste caso, dizemos que a solução explode (blow-up, em inglês). Devido ao caráter introdutório deste trabalho, nos dedicaremos ao caso em que $f$ independe do gradiente. O caso mais difícil, em que os efeitos de convecção são levados em consideração, vem recebendo atenção somente nos últimos anos [5, 20].

Antes de prosseguir, vejamos uma equação de reação pura, $u_{t}=f(u), u(0)=u_{0}$, com $f(u) \geq 0$. Esta solução tem blow-up em tempo finito se, e somente se,

$$
\int_{u_{0}}^{\infty} f^{-1}(s) d s<\infty
$$

A difusão $\Delta u$ e a condição de fronteira $u(x, t)=0$ sobre $\partial D \times \mathbb{R}^{N}$ contribuem para diminuição da solução. Como no problema (1.1), todos os mecanismos tais como reação, difusão e a condição 
de fronteira atuam simultaneamente, é natural, pois, perguntar qual desses prevalece.

O fenômeno de blow-up para equações de reação-difusão são estudados desde o pioneiro trabalho de Kaplan [16]. Ele mostrou que para funções convexas safisfazendo (1.2), a difusão não pode evitar blow-up se o dado inicial é suficientemente grande. O próximo resultado fundamental nesta linha é dado por Fujita em [9, 10], que prova que o problema de Cauchy, para $D=\mathbb{R}^{N}$ e $f(u)=u^{p}$, não tem solução global não trivial e positiva se $1<p<1+2 / N$. Toda solução com dado inicial arbitrariamente pequeno tem blow-up em tempo finito. O mesmo continua válido para $p<1+2 / N$, como foi provado por Hayahawa em [13]. Quando $p>1+2 / N$, soluções com dados iniciais pequenos tendem a zero quando $t$ cresce.

Questões básicas. A interpretação física do blow-up é dada como um dramático aumento de temperatura que leva à ignição de uma reação química. Neste contexto, surgem as seguintes questões:

\section{Blow-up ocorre?}

\section{Quando?}

3. Onde?

\section{Como?}

5. O que ocorre após o blow-up?

6. É possivel calcular numericamente a solução de blow-up?

As duas primeiras questões estão razoavelmente bem discutidas. Durante a duas últimas décadas, grandes progressos foram obtidos no estabelecimento de critérios de blow-up. Os resultados de Kaplan e Fujita foram generalizados para vários casos. Muito mais difícil é a localização do blow-up (terceira questão) e a discussão do comportamento assintótico da solução de blow-up (quarta questão) dada pelas seguintes sub-questões: (4.i) com que taxa u diverge quando $t \rightarrow T^{-}$ex se aproxima dos pontos de blow-up? (4.ii) qual é o perfil de u(x,t) quando $t \rightarrow T^{-}$nos pontos que não são de blow-up? Se $u$ pode ser continuada após o tempo de blowup, então a ignição de fato ainda não ocorreu. Daí reside a importância da questão 5. A última questão refere-se aos métodos numéricos para detectar o fenômeno de blow-up e calcular a solução de blow-up, o tempo de blow-up e o perfil. Como pode-se ver em [3], esta questão está praticamente restrita ao caso unidimesional e muitos problemas permanecem abertos no tratamento numérico de problemas de blow-up.

O presente trabalho toca de leve a teoria de blow-up em equações diferenciais, que vem se desenvolvendo intensamente nas últimas décadas. Citamos o artigo [1] para mais informações 
e referências. Neste trabalho não abordamos todas as questões acima (o que seria por demais pretencioso), consideramos apenas as questões 3 e 4. Para isso foi necessário utilizar resultados de Análise Funcional, Teoria da Medida e Integração e Espaços de Sobolev. Começamos tratando um problema de evolução num espaço de Banach, via Teoria de Semigrupos de Operadores Lineares, o que constituiu parte fundamental do trabalho, tendo em vista o conjunto de conceitos e idéias que foram introduzidos. Na etapa final nos dedicamos ao problema de blow-up, usando [8] como principal bibliografia. Neste ponto lançamos mão dos resultados de existência estudados na primeira fase. Desta forma, o trabalho foi organizado da seguinte forma: no Capítulo 1, apresentamos diversas definições e resultados da Teoria de Semigrupos, deixamos de demonstrar alguns dos resultados apresentados a fim de tornar a leitura menos cansativa. Dedicamos o Capítulo 2 ao estudo detalhado da Teoria de Potências Fracionárias, parte fundamental ao desenvolvimento do capítulo seguinte. No Capítulo 3 apresentamos alguns resultados de existência e regularidade de solução para os problemas de Cauchy não-homogêneo e semilinear. Finalizando o trabalho, no Capítulo 4 apresentamos diversos resultados referentes à localização dos pontos de blow-up do problema de valor inicial e de fronteira

$$
\begin{array}{rll}
u_{t}=\Delta u+f(u) \quad \text { em } & \Omega \times(0, T), \\
u(x, 0)=\phi(x) & \text { se } & x \in \Omega, \\
u(x, t)=0 & \text { se } & x \in \partial \Omega, 0<t<T,
\end{array}
$$

onde $\Omega$ é um domínio limitado em $\mathbb{R}^{n}$ com bordo $C^{2}$,

$$
\begin{array}{r}
f \in C^{1}, \quad f(s)>0 \text { se } s \geq 0, \quad f(s)=0 \text { se } s<0, \\
\phi \in C^{1}(\bar{\Omega}), \quad \phi \geq 0 \quad \text { (não identicamente nula), } \phi=0 \text { sobre } \partial \Omega .
\end{array}
$$

Nos dedicamos ao estudo do conjunto de pontos de blow-up e no comportamento de $u$ nesses pontos. Nossos resultados foram divididos de acordo com os dois seguintes casos:

1. o caso simétrico, e

2. o caso não-simétrico.

No caso simétrico, $\Omega$ é uma bola e $\phi, u(., t)$ são funções radiais. É admitido que $\phi_{r} \leq 0$. Provamos então, para uma classe geral de funções $f$, que o conjunto de blow-up do problema (0.1)-(0.3) consiste de um único ponto $x=0$. Para a função

$$
f(u)=u^{p} \quad, \quad p>1
$$


obtemos também as estimativas

$$
\begin{aligned}
& |u(r, t)| \leq \frac{c}{r^{\frac{2}{\gamma-1}}} \text { para qualquer } 1<\gamma<p, \\
& \limsup _{t \rightarrow T^{-}}\|u(., t)\|_{L^{q}(\Omega)}<\infty \quad \text { se } q<\frac{n(p-1)}{2}, \\
& \liminf _{t \rightarrow T^{-}}\|u(., t)\|_{L^{q}(\Omega)}=\infty \quad \text { se } q>\frac{n(p-1)}{2},
\end{aligned}
$$

e se $\Delta \phi+f(\phi) \geq 0$ e $n=1,2$ ou se $p \leq \frac{n+2}{n-2}$ quando $n \geq 3$, obtemos que

$$
(T-t)^{\beta} u(r, t) \rightarrow \beta^{\beta} \quad \text { quando } t \uparrow T \quad\left(\beta=\frac{1}{p-1}\right)
$$

com a condição de que $r \leq C(T-t)^{\frac{1}{2}}$ para algum $C>0$.

No caso-não simétrico admitimos que $\Omega$ é um domínio convexo e para alguns resultados é pedido que $\Delta \phi+f(\phi) \geq 0$ (e então $u_{t} \geq 0$ pelo Princípio do Máximo). Provamos que o conjunto de blow-up do problema (0.1)-(0.3) é um subconjunto compacto de $\Omega$ e que para $f$ dada por $(0.4)$,

$$
u(x, t) \leq \frac{C}{(T-t)^{\frac{1}{p-1}}} \quad \text { para todo } x \in \Omega
$$

Provamos também, no caso não-simétrico, que para $f$ dada por (0.4)

$$
\liminf _{t \rightarrow T^{-}}\|u(., t)\|_{L^{q}(\Omega)}=\infty \quad \text { se } q>\frac{n(p-1)}{2} .
$$

A maioria de nossos resultados são afirmados para uma função geral $f$ e então especializado para $f$ como em (0.4). As condições sobre a função geral $f$ são afirmadas em termos de funções auxiliares $F$. 


\section{Capítulo 1}

\section{Preliminares}

Neste primeiro capítulo apresentamos algumas definições e resultados preliminares ao desenvolvimento deste trabalho. As principais referências utilizadas foram [15] e [19].

\section{$1.1 \quad$ Semigrupos e seus geradores}

Recorde que se $E$ e $F$ são espaços de Banach sobre um corpo $\mathbb{K}(\mathbb{K}=\mathbb{R}$ ou $\mathbb{K}=\mathbb{C})$ denotamos por $L(E, F)$ o espaço dos operadores lineares e contínuos de $E$ em $F$ com a norma usual; isto é, para $T \in L(E, F)$,

$$
\|T\|_{L(E, F)}=\sup _{\substack{e \in E \\ e \neq 0}} \frac{\|T e\|_{F}}{\|e\|_{E}} .
$$

No caso particular, em que $E=F$ escrevemos $L(E)$ para denotar $L(E, E)$. Seja $E^{*}$ o dual topológico de $E$; isto é, $E^{*}=L(E, \mathbb{K})$ com a topologia dada pela norma acima. Denotamos o valor de $e^{*} \in E^{*}$ em $e \in E$ por $\left\langle e^{*}, e\right\rangle$ ou $\left\langle e, e^{*}\right\rangle$.

Definição 1.1Um semigrupo de operadores lineares em $E$ é uma família $\{T(t): t \geq 0\} \subset$ $L(E)$ tal que

(i) $T(0)=I_{E}$,

(ii) $T(t+s)=T(t) T(s)$, para todo $t, s \geq 0$.

Se adicionalmente

(iii) $\left\|T(t)-I_{E}\right\|_{L(E)} \rightarrow 0$ quando $t \rightarrow 0^{+}$, dizemos que o semigrupo é uniformemente contínuo

(iv) $\|T(t) e-e\|_{E} \rightarrow 0$ quando $t \rightarrow 0^{+}, \forall e \in E$, dizemos que o semigrupo é fortemente contínuo. 
Todo semigrupo fortemente contínuo possui uma dominação exponencial que é dada no teorema a seguir.

Teorema 1.1 Suponha que $\{T(t): t \geq 0\} \subset L(E)$ é um semigrupo fortemente contínuo. Então, existem $M \geq 1$ e $\beta \in \mathbb{R}$ tais que

$$
\|T(t)\|_{L(E)} \leq M \mathbf{e}^{\beta t}, \quad \forall t \geq 0
$$

Para qualquer $\ell>0$ podemos escolher $\beta \geq \frac{1}{\ell} \log \|T(\ell)\|_{L(E)}$ e então escolher $M$.

Prova. Primeiramente note que $\sup _{t \in[0, \eta]}\|T(t)\|_{L(E)}<\infty$ para algum $\eta>0$, pois caso contrário existiria uma sequência $t_{n} \rightarrow 0^{+}$tal que $\left\|T\left(t_{n}\right)\right\|_{L(E)} \rightarrow \infty$. Mas $\left\{T\left(t_{n}\right) e\right\}_{n \geq 1}$ é limitada para cada $e \in E$. Portanto, do Princípio da Limitação Uniforme, $\left\{\left\|T\left(t_{n}\right)\right\|_{L(E)}\right\}_{n \geq 1}$ é limitada, contrariando a hipótese.

Assim, escolha $\ell>0$ tal que $\sup \left\{\|T(t)\|_{L(E)}, 0 \leq t \leq \ell\right\}=M<\infty$. Como $\|T(0)\|_{L(E)}=1$, temos $M \geq 1$. Seja $\beta \geq \frac{1}{\ell} \log \left\{\|T(\ell)\|_{L(E)}\right\}$. Então, dado $t \geq 0$, temos $t=n l+\delta \operatorname{com} 0 \leq \delta<l$ e $n \in \mathbb{N}$. Pelas propriedades de semigrupos

$$
\begin{aligned}
\|T(t)\|_{L(E)} & =\|T(n l+\delta)\|_{L(E)}=\|T(n l) T(\delta)\|_{L(E)}=\left\|T(l)^{n} T(\delta)\right\|_{L(E)} \leq \\
& \leq M^{n+1}=M M^{n} \leq M M^{t / l}=M \mathbf{e}^{\beta t}, n=0,1,2 \ldots,
\end{aligned}
$$

onde usamos o fato de que $n=\frac{t-\delta}{l}<\frac{t}{l}$ e $\mathbf{e}^{\beta t}=\mathbf{e}^{t\left(\frac{1}{l} \log M\right)}=M^{\frac{t}{l}}$.

Definição 1.2 Seja $A: D(A) \subset E \longrightarrow E$ um operador linear, o conjunto

$$
\left\{\lambda \in \mathbb{C}: \overline{R(\lambda I-A)}=E,(\lambda I-A)^{-1} \text { existe e é limitado sobre } R(\lambda I-A)\right\}
$$

é chamado conjunto resolvente do operador $A$ e é denotado por $\rho(A)$. O conjunto $\sigma(A)=\mathbb{C} / \rho(A)$ é chamado espectro do operador $A$.

Definição 1.3 Se $\{T(t): t \geq 0\} \subset L(E)$ é um semigrupo fortemente contínuo de operadores lineares, seu gerador infinitesimal é o operador definido por $A: D(A) \subset E \rightarrow E$, onde

$$
D(A)=\left\{e \in E: \lim _{t \rightarrow 0^{+}} \frac{T(t) e-e}{t} \text { existe }\right\}, \quad A e=\lim _{t \rightarrow 0^{+}} \frac{T(t) e-e}{t} .
$$

O resultado a seguir coleta alguns fatos importantes sobre semigrupos fortemente contínuos. Sua prova é simples e é apresentada apenas para conveniência do leitor. 
Teorema 1.2 Suponha que $\{T(t): t \geq 0\} \subset L(E)$ é um semigrupo fortemente contínuo.

1. Para qualquer $e \in E, t \mapsto T(t) e$ é contínuo para $t \geq 0$.

2. $t \mapsto\|T(t)\|_{L(E)}$ é semicontínua inferiormente e portanto mensurável.

3. Seja A o gerador infinitesimal de $T(t)$. Então, A é densamente definido e fechado. Para $e \in D(A), t \mapsto T(t) e$ é continuamente diferenciável $e$

$$
\frac{d}{d t} T(t) e=A T(t) e=T(t) A e, \quad t>0 .
$$

4. $\cap_{m \geq 1} D\left(A^{m}\right)$ é denso em $E$.

5. Para $\operatorname{Re} \lambda>\beta$ e $\beta$ dado no Teorema 1.1, $\lambda$ está no resolvente $\rho(A)$ de $A$ e

$$
(\lambda-A)^{-1} e=\int_{0}^{\infty} \mathbf{e}^{-\lambda t} T(t) e d t, \quad \forall e \in E .
$$

Prova. (1) A continuidade de $t \mapsto T(t) e$ é uma consequência de Teorema 1.1 e de

$$
\|T(t+h) e-T(t) e\|_{E}=\|(T(h)-I) T(t) e\|_{E} \rightarrow 0, \quad \text { quando } h \rightarrow 0^{+}, t>0, e \in E,
$$

$\|T(t) e-T(t-h) e\|_{E} \leq \|\left(T(t-h)\left\|_{L(E)}\right\| T(h) e-e \|_{E} \rightarrow 0, \quad\right.$ quando $h \rightarrow 0^{+}, t>0, e \in E$.

(2) Mostremos que $\mathcal{O}=\left\{t \geq 0:\|T(t)\|_{L(E)}>b\right\}$ é aberto em $[0, \infty)$ para cada $b$, o que implica a afirmativa. Para tanto mostremos que dado $t_{0} \in \mathcal{O}$ existe vizinhança $\mathcal{U}$ de $t_{0}$ tal que $\mathcal{U} \subset \mathcal{O}$. Mas $\left\|T\left(t_{0}\right)\right\|_{L(E)}>b$ implica que existe $e \in E$ com $\|e\|_{E}=1$ tal que $\left\|T\left(t_{0}\right) e\right\|>b$. Segue de (1) que $\|T(t) e\|>b$ para $t$ em uma vizinhança de $t_{0}$. Logo $\|T(t)\|_{L(E)}>b$ para $t$ em uma vizinhança de $t_{0}$ e o resultado segue.

(3) Seja $e \in E$ e para $\epsilon>0, e_{\epsilon}=\frac{1}{\epsilon} \int_{0}^{\epsilon} T(t) e d t$; então segue da continuidade de $t \mapsto T(t) e$ que $e_{\epsilon} \rightarrow e$ quando $\epsilon \rightarrow 0^{+}$. Além disso, $e_{\epsilon} \in D(A)$, pois para $h>0$

$$
h^{-1}\left(T(h) e_{\epsilon}-e_{\epsilon}\right)=\frac{1}{\epsilon h}\left\{\int_{\epsilon}^{\epsilon+h} T(t) e d t-\int_{0}^{h} T(t) e d t\right\} \rightarrow \frac{1}{\epsilon}(T(\epsilon) e-e),
$$

quando $h \rightarrow 0^{+}$. Logo $e_{\epsilon} \in D(A)$ e $D(A)$ é denso em $E$. Será uma consequência imediata de (5) que $A$ é fechado pois $(\lambda-A)^{-1} \in L(E)$. Se $e \in D(A)$ é claro que

$$
\frac{d^{+}}{d t} T(t) e=\lim _{h \rightarrow 0^{+}} \frac{1}{h}\{T(t+h) e-T(t) e\}=A T(t) e=T(t) A e
$$

é contínua e toda função com derivada à direita contínua é diferenciável.

(4) Seja $\phi: \mathbb{R} \rightarrow \mathbb{R}$ uma função em $C^{\infty}(\mathbb{R})$ com $\phi(t)=0$ em uma vizinhança de $t=0$ e também para $t$ suficientemente grande. Seja $e \in E$ e $f=\int_{0}^{\infty} \phi(t) T(t) e d t$. Segue facilmente de 
$h^{-1}(T(h) f-f)=h^{-1} \int_{h}^{\infty}(\phi(t-h)-\phi(t)) T(t) e d t$ que $f \in D(A)$ e que $A f=-\int_{0}^{\infty} \phi^{\prime}(t) T(t) e d t$. Como $-\phi^{\prime}$ satisfaz as mesmas condições que $\phi$, repetindo o argumento anterior temos que $f \in D\left(A^{m}\right)$ e que

$$
A^{m} f=(-1)^{m} \int_{0}^{\infty} \phi^{(m)}(t) T(t) e d t
$$

para todo $m \geq 1$. Consequentemente $f \in \cap_{m \geq 1} D\left(A^{m}\right)$. Para mostrar que tal conjunto de pontos é denso em $E$, escolha $\phi$ acima satisfazendo também $\int_{0}^{\infty} \phi(t) d t=1$; então se, $f_{n}=\int_{0}^{\infty} n \phi(n t) T(t) e d t=\int_{0}^{\infty} \phi(s) T(s / n) e d s, n=1,2,3, \cdots$ e temos que $f_{n} \in \cap_{m \geq 1} D\left(A^{m}\right)$ e $f_{n} \rightarrow e$ quando $n \rightarrow \infty$.

(5)Defina $R(\lambda) \in L(E)$ por

$$
R(\lambda) e=\int_{0}^{\infty} \mathbf{e}^{-\lambda t} T(t) e d t
$$

e note que $\|R(\lambda)\|_{L(E)} \leq \frac{M}{\operatorname{Re} \lambda-\beta}$, se $\operatorname{Re} \lambda>\beta$ e $\|T(t)\|_{L(E)} \leq M \mathbf{e}^{\beta t}$. Seja $e \in E$ e $h>0$

$$
\begin{aligned}
h^{-1}(T(h)-I) R(\lambda) e & =R(\lambda) \frac{T(h) e-e}{h}= \\
& =h^{-1}\left[\int_{h}^{\infty} \mathbf{e}^{-\lambda t+\lambda h} T(t) e-\int_{0}^{\infty} \mathbf{e}^{-\lambda t} T(t) e\right]= \\
& =h^{-1}\left[-\int_{0}^{h} \mathbf{e}^{\lambda(h-t)} T(t) e+\int_{0}^{\infty}\left(\mathbf{e}^{\lambda h}-1\right) \mathbf{e}^{-\lambda t} T(t) e\right] \\
& \rightarrow-e+\lambda R(\lambda) e, \quad \text { quando } h \rightarrow 0^{+} .
\end{aligned}
$$

Portanto $R(\lambda) e \in D(A)$ e $(\lambda-A) R(\lambda) e=e$, e $\lambda-A$ é sobrejetivo. Também, se $e \in D(A)$ então, como $A R(\lambda) e=R(\lambda) A e$, por (1.1) vemos que $(\lambda-A) R(\lambda) e=e=R(\lambda)(\lambda-A) e, e \in D(A) \mathrm{e}$ $\lambda-A$ é também um-a-um, portanto uma bijeção de $D(A)$ sobre $E$ com inversa limitada $R(\lambda)$ e a prova está completa.

Teorema 1.3 Sejam $\{T(t), t \geq 0\}$ e $\{S(t), t \geq 0\}$ semigrupos fortemente contínuos com geradores infinitesimais $A$ e $B$ respectivamente. Se $A=B$ então $T(t)=S(t), t \geq 0$.

Prova. Seja $e \in D(A)=D(B)$. Do Teorema 1.2 segue facilmente que a função $s \mapsto T(t-s) S(s) e$ é diferenciável e que

$$
\begin{aligned}
\frac{d}{d s} T(t-s) S(s) e & =-A T(t-s) S(s) e+T(t-s) B S(s) e= \\
& =-T(t-s) A S(s) e+T(t-s) B S(s) e=0 .
\end{aligned}
$$


Portanto $s \mapsto T(t-s) S(s) e$ é constante e em particular seus valores em $s=0$ e $s=t$ são os mesmos, isto é $T(t) e=S(t) e$. Isto vale para todo $e \in D(A)$ e como $D(A)$ é denso em $E$ e $S(t)$, $T(t)$ são limitados, $T(t) e=S(t) e$ para todo $e \in E$.

\subsection{O Teorema de Hille-Yosida}

Teorema 1.4 (Hille-Yosida) Suponha que $A: D(A) \subset E \rightarrow E$ é um operador linear. Então os fatos seguintes são equivalentes

(i) A é o gerador infinitesimal de um semigrupo fortemente contínuo $\{T(t): t \geq 0\} \subset L(E)$ tal que

$$
\|T(t)\|_{L(E)} \leq \mathbf{e}^{\omega t}, \quad \forall t \geq 0
$$

(ii) A é um operador linear fechado, densamente definido cujo conjunto resolvente contém $(\omega, \infty) e$

$$
\left\|(\lambda-A)^{-1}\right\|_{L(E)} \leq \frac{1}{\lambda-\omega}, \quad \forall \lambda>\omega .
$$

Prova. Seque do Teorema $1.2(3)$, que A é operador linear fechado e densamente definido. Além disso, como $\|T(t)\|_{L(E)} \leq 1 . \mathrm{e}^{\omega t}, t \geq 0$, segue do Teorema 1.2 (5) que $\{\lambda \in \mathbb{C}: \operatorname{Re} \lambda>\omega\} \subset \rho(A)$ (em particular, se $\lambda>\omega$ então $\lambda \in \rho(A))$ e que

$$
\left\|(\lambda-A)^{-1} e\right\|_{E} \leq \int_{0}^{\infty} \mathbf{e}^{-\lambda t}\|T(t) e\|_{E} d t \leq \frac{1}{\lambda-\omega}\|e\|_{E}
$$

se $\lambda>\omega$.

Por outro lado, note que $T(t) \mathbf{e}^{-\omega t}=T_{1}(t)$ é um semigrupo com $\left\|T_{1}(t)\right\|_{L(E)} \leq 1$ (chamado semigrupo de contrações) e o gerador de $T_{1}(t)$ é $A-\omega$ logo é suficiente tratar o caso $\omega=0$. Admita que (ii) vale com $\omega=0$. Para $\lambda>0$

$$
\left\|\lambda(\lambda-A)^{-1}\right\|_{L(E)} \leq 1, \quad \lambda(\lambda-A)^{-1}=\left(I-\lambda^{-1} A\right)^{-1}=I+A(\lambda-A)^{-1}
$$

então $e \in D(A)$ implica

$$
\left\|\lambda(\lambda-A)^{-1} e-e\right\|_{E}=\left\|(\lambda-A)^{-1} A e\right\|_{E} \leq \lambda^{-1}\|A e\|_{E} \rightarrow 0
$$

quando $\lambda \rightarrow \infty$ e, como $A$ é densamente definido,

$$
\lambda(\lambda-A)^{-1} e=\left(I-\lambda^{-1} A\right)^{-1} e \rightarrow e \text { quando } \lambda \rightarrow \infty
$$

para cada $e \in E$. Defina $A_{\lambda}=A\left(I-\lambda^{-1} A\right)^{-1}, \lambda>0$ então $A_{\lambda} \in L(E)$,

$$
\left\|A_{\lambda}\right\|_{L(E)}=\lambda\left\|A(\lambda-A)^{-1}\right\|_{L(E)} \leq 2 \lambda
$$


e se $e \in D(A), A_{\lambda} e \rightarrow A e$ quando $\lambda \rightarrow \infty$. $A_{\lambda}$ é a Aproximação de Yosida do gerador $A$. Obtemos $T(t)$ como o limite de $\mathbf{e}^{t A_{\lambda}}$ quando $\lambda \rightarrow \infty$. Primeiro note que

$$
A_{\lambda}=\lambda^{2}(\lambda-A)^{-1}-\lambda I_{E}
$$

$\log 0$

$$
\begin{aligned}
\left\|\mathbf{e}^{t A_{\lambda}}\right\|_{L(E)} & =\left\|\mathbf{e}^{-\lambda t} \mathbf{e}^{t \lambda^{2}(\lambda-A)^{-1}}\right\|_{L(E)} \\
& \leq \mathbf{e}^{-\lambda t} \mathbf{e}^{t \lambda^{2}\left\|(\lambda-A)^{-1}\right\|_{L(E)}} \leq 1
\end{aligned}
$$

e para qualquer $\lambda, \mu>0($ e $t>0)$, desde que $A_{\lambda} A_{\mu}=A_{\mu} A_{\lambda}$,

$$
\begin{aligned}
\left\|\mathbf{e}^{t A_{\lambda}} e-\mathbf{e}^{t A_{\mu}} e\right\|_{E} & =\left\|\int_{0}^{1} \frac{d}{d s}\left(\mathbf{e}^{t s A_{\lambda}} \mathbf{e}^{t(1-s) A_{\mu}} e\right) d s\right\|_{E} \\
& \leq \int_{0}^{1} t\left\|\mathbf{e}^{t s A_{\lambda}} \mathbf{e}^{t(1-s) A_{\mu}}\left(A_{\lambda} e-A_{\mu} e\right)\right\|_{E} d s \\
& \leq t\left\|A_{\lambda} e-A_{\mu} e\right\|_{E} .
\end{aligned}
$$

Portanto para $e \in D(A), T(t) e \equiv \lim _{\lambda \rightarrow \infty} \mathbf{e}^{t A_{\lambda}} e$ existe uniformemente para $0 \leq t \leq t_{0}$, qualquer $t_{0}>0, t \rightarrow T(t) e$ é contínuo para $t \geq 0, T(t) e \rightarrow e$ em $E$ quando $t \rightarrow 0^{+}, T(t)(T(s) e)=T(t+s) e$ para $t, s \geq 0$ e $\|T(t) e\|_{E} \leq\|e\|_{E}$. Podemos definir de forma única $T(t) \in L(E)$ para cada $t \geq 0$.

Se $e \in E, \epsilon>0$ dados. Então existem $e_{1} \in D(A)$ e $\delta>0$ tais que, $\left\|e_{1}-e\right\|_{E}<\epsilon / 3$ e $\left\|T(t) e_{1}-e_{1}\right\|_{E}<\epsilon / 3,0 \leq t \leq \delta$. Segue que

$$
\|T(t) e-e\|_{E} \leq\left\|T(t)\left(e-e_{1}\right)\right\|_{E}+\left\|T(t) e_{1}-e_{1}\right\|_{E}+\left\|e_{1}-e\right\|_{E}<\epsilon
$$

Portanto $\{T(t), t \geq 0\} \subset L(E)$ é um semigrupo fortemente contínuo. Só resta provar que $A$ é o seu gerador.

Seja $e \in D\left(A^{2}\right)$, então

$$
\begin{aligned}
T(t) e-e & =\lim _{\lambda \rightarrow \infty}\left(\mathbf{e}^{t A_{\lambda}} e-e\right)=\lim _{\lambda \rightarrow \infty} \int_{0}^{t} \mathbf{e}^{s A_{\lambda}} A_{\lambda} e d s \\
& =\int_{0}^{t} T(s) A e d s .
\end{aligned}
$$

Tomando limites, isto vale também para $e \in D(A)$ (isto é feito da seguinte forma: tomamos $e \in D(A)$ e $\left\{f_{n}\right\} \subset D(A)$ tal que $f_{n} \rightarrow A e$ então $D\left(A^{2}\right) \ni g_{n}=A^{-1} f_{n} \rightarrow e$ e $D\left(A^{2}\right) \ni g_{n} \rightarrow e$, $\left.A g_{n} \rightarrow A e\right)$. 
Agora $\frac{1}{t}(T(t) e-e)=\frac{1}{t} \int_{0}^{t} T(s)$ Aeds $\rightarrow$ Ae quando $t \rightarrow 0^{+}$, para qualquer $e \in D(A)$. Portanto o gerador $B$ de $T(t)$ deve ser uma extensão de $A$ (isto é $D(B) \supset D(A)$ e $B e=A e$ quando $e \in D(A))$. Mas, por hipótese, $1 \in \rho(A)$ e, do fato que $B$ é o gerador de um semigrupo fortemente contínuo de contrações, $1 \in \rho(B)$, então

$$
E=(I-A) D(A)=(I-B) D(A),
$$

então $(I-B) D(A)=E=(I-B) D(B), D(A)=R\left((I-B)^{-1}\right)=D(B)$, e segue que $A=B$ e a prova está completa.

Ambas condições (i) e (ii) dependem da escolha da norma em E. Daremos uma formulação independente da norma, mas na prática devemos usualmente procurar normas especiais para a qual este teorema se aplique.

Lema 1.1 Suponha que A é um operador linear cujo conjunto resolvente contém $(0, \infty)$ e que satisfaz

$$
\left\|(\lambda-A)^{-n}\right\|_{L(E)} \leq M \lambda^{-n}, \quad n \geq 1, \lambda>0 .
$$

Então existe uma norma $|\cdot|_{E}$ em E tal que

$$
\|e\|_{E} \leq|e|_{E} \leq M\|e\|_{E}, \quad \forall e \in E
$$

$$
\left|(\lambda-A)^{-1} e\right|_{E} \leq \lambda^{-1}|e|_{E}, \quad \forall e \in E, \lambda>0 .
$$

Teorema 1.5 (Forma Geral do Teorema de Hille-Yosida) Seja $A: D(A) \subset E \rightarrow E$ um operador linear. As seguintes afirmativas são equivalente

(i) A é o gerador infinitesimal de um semigrupo fortemente contínuo $\{T(t): t \geq 0\} \subset L(E)$ tal que

$$
\|T(t)\|_{L(E)} \leq M \mathbf{e}^{\beta t}, \quad \forall t \geq 0
$$

(ii) A é fechado, densamente definido, o conjunto resolvente de $A$ contém $(\beta, \infty) e$

$$
\left\|(\lambda-A)^{-n}\right\|_{L(E)} \leq M(\lambda-\beta)^{-n}, \quad \forall \lambda>\beta, n=1,2, \cdots .
$$




\subsection{O Teorema de Lumer-Phillips}

Definição 1.4 Seja $E$ um espaço de Banach sobre $\mathbb{K}$ com norma $\|\cdot\|_{E}$ e seja $E^{*}=L(E, \mathbb{K})$ o seu dual topológico com a norma usual $\|\cdot\|_{E^{*}}\left(\left\|e^{*}\right\|_{E^{*}}=\sup \left\{\operatorname{Re}\left\langle e^{*}, e\right\rangle:\|e\|_{E} \leq 1\right\}\right)$. A aplicação dualidade $J: E \rightarrow 2^{E^{*}}$ é uma função multívoca definida por

$$
J(e)=\left\{e^{*} \in E^{*}: \operatorname{Re}\left\langle e^{*}, e\right\rangle=\|e\|_{E}^{2},\left\|e^{*}\right\|_{E^{*}}=\|e\|_{E}\right\} .
$$

$J(e) \neq \varnothing$, pelo Teorema de Hahn-Banach.

Definição 1.5 Um operador linear $A: D(A) \subset E \rightarrow E$ é dissipativo se para cada e $\in D(A)$ existe $e^{*} \in J(e)$ tal que $\operatorname{Re}\left\langle e^{*}, A e\right\rangle \leq 0$.

Observação 1.1 Se $\{T(t): t \geq 0\} \subset L(E)$ é um semigrupo fortemente contínuo tal que

$$
\|T(t)\| \leq 1, \forall t \leq 0
$$

então dizemos que $T(t)$ é um semigrupo fortemente contínuo de contrações.

Lema 1.2 O operador linear A é dissipativo se, e somente se,

$$
\|(\lambda-A) e\|_{E} \geq \lambda\|e\|_{E}
$$

para todo $e \in D(A)$ e $\lambda>0$.

Prova. Seja $A$ dissipativo. Se $e=0$, o resultado é óbvio. Sejam $\lambda>0,0 \neq e \in D(A)$ e $e^{*} \in J(e)$ tal que $\operatorname{Re}\left\langle A e, e^{*}\right\rangle \leq 0$. Então

$$
\|\lambda e-A e\|_{E}\|e\|_{E} \geq\left|\left\langle\lambda e-A e, e^{*}\right\rangle\right| \geq \operatorname{Re}\left\langle\lambda e-A e, e^{*}\right\rangle \geq \lambda\|e\|_{E}^{2}
$$

e (1.3) segue. Reciprocamente, seja $e \in D(A)$ e admita que $\lambda\|e\|_{E} \leq\|\lambda e-A e\|_{E}$ para todo $\lambda>0$. Se $e=0$, qualquer $e^{*} \in J(e)$ satisfaz $\operatorname{Re}\left\langle A e, e^{*}\right\rangle \leq 0$. Podemos então assumir $0 \neq e \in D(A)$. Seja $f_{\lambda}^{*} \in J(\lambda e-A e), \lambda>0$. Então temos

$$
0<\lambda\|e\|_{E} \leq\|\lambda e-A e\|_{E}=\left\|f_{\lambda}^{*}\right\|_{E^{*}}
$$

e podemos então definir $g_{\lambda}^{*}=f_{\lambda}^{*} /\left\|f_{\lambda}^{*}\right\|_{E^{*}}$. Daí,

$$
\lambda\|e\|_{E} \leq\|\lambda e-A e\|_{E}=\left\langle\lambda e-A e, g_{\lambda}^{*}\right\rangle=\lambda \operatorname{Re}\left\langle e, g_{\lambda}^{*}\right\rangle-\operatorname{Re}\left\langle A e, g_{\lambda}^{*}\right\rangle \leq \lambda\|e\|_{E}-\operatorname{Re}\left\langle A e, g_{\lambda}^{*}\right\rangle,
$$

donde segue que $\operatorname{Re}\left\langle A e, g_{\lambda}^{*}\right\rangle \leq 0$.

Como a bola unitária de $E^{*}$ é compacta na topologia fraca* existem $g^{*} \in E^{*},\left\|g^{*}\right\|_{E^{*}} \leq 1$, e sequência $\lambda_{n} \rightarrow \infty$ tais que $g_{\lambda_{n}}^{*} \stackrel{w^{*}}{\longrightarrow} g^{*}$. De (1.4) segue que $\operatorname{Re}\left\langle A e, g^{*}\right\rangle \leq 0$ e $\operatorname{Re}\left\langle e, g^{*}\right\rangle \geq\|e\|_{E}$. 
$\operatorname{Mas} \operatorname{Re}\left\langle e, g^{*}\right\rangle \leq\left|\left\langle e, g^{*}\right\rangle\right| \leq\|e\|_{E}$ e portanto $\operatorname{Re}\left\langle e, g^{*}\right\rangle=\|e\|_{E}$. Tomando $e^{*}=\|e\|_{E} g^{*}$ temos $e^{*} \in J(e)$ e $\operatorname{Re}\left\langle A e, e^{*}\right\rangle \leq 0$. Portanto, para todo $e \in D(A)$ existe $e^{*} \in J(e)$ tal que $\operatorname{Re}\left\langle A e, e^{*}\right\rangle \leq 0$ e $A$ é dissipativo.

Teorema 1.6 (Lumer-Phillips) Suponha que A é um operador linear densamente definido em um espaço de Banach E.

(i) Se A é o gerador infinitesimal de um semigrupo fortemente contínuo de contrações, então $A$ é dissipativo (de fato, $\operatorname{Re}\left\langle e^{*}, A e\right\rangle \leq 0$ para todo $\left.e^{*} \in J(e)\right)$ e $R(\lambda-A)=E$ para todo $\lambda>0$,

(ii) Se $A$ é dissipativo e $R\left(\lambda_{0}-A\right)=E$ para algum $\lambda_{0}>0$, então $A$ é o gerador de um semigrupo fortemente contínuo de contrações.

Prova. (i) Se $A$ gera $\{T(t): t \geq 0\}$ com $\|T(t)\|_{L(E)} \leq 1$ segue do Teorema de Hille-Yosida que $(0, \infty) \subset \rho(A)$ e então $R(\lambda-A)=E$ para todo $\lambda>0$. Além disso, para quaisquer $e \in D(A)$, $e^{*} \in J(e), t>0$,

$$
\left|\left\langle e^{*}, T(t) e\right\rangle\right| \leq\left\|e^{*}\right\|_{E^{*}}\|T(t) e\|_{E} \leq\|e\|_{E}^{2}
$$

e então

$$
\operatorname{Re}\left\langle e^{*}, \frac{T(t) e-e}{t}\right\rangle=\frac{1}{t}\left\{\operatorname{Re}\left\langle e^{*}, T(t) e\right\rangle-\|e\|_{E}^{2}\right\} \leq 0 .
$$

Fazendo $t \rightarrow 0^{+}$, segue que se $e \in D(A), \operatorname{Re}\left\langle e^{*}, A e\right\rangle \leq 0$, o que mostra que A é dissipativo.

(ii) Suponhamos que A seja dissipativo e que exista $\lambda_{0}>0$ tal que $R\left(\lambda_{0}-A\right)=E$. Se $\lambda>0$ e $e \in D(A)$, segue do Lema 1.2 que

$$
\|(\lambda-A) e\|_{E} \geq \lambda\|e\|_{E}
$$

Assim, temos $R\left(\lambda_{0}-A\right)=E$ e $\left\|\left(\lambda_{0}-A\right) e\right\|_{E} \geq \lambda_{0}\|e\|_{E}$ para $e \in D(A)$. Logo $\lambda_{0}$ está no conjunto resolvente de $A$ e $A$ é fechado. Resta verificar que $R(\lambda-A)=E$ para $\lambda>0$ e então $\lambda \in \rho(A)$ para $\lambda>0$, isto é, $(0, \infty) \subset \rho(A)$. Para tanto, considere o conjunto $\Lambda=\{\lambda \in \rho(A) \cap \mathbb{R}: \lambda>0\}$. Como $\lambda_{0} \in \Lambda$, então $\Lambda \neq \emptyset$. Além disso, $\Lambda$ é um conjunto aberto em $(0, \infty)$ já que $\rho(A)$ é aberto. Provaremos que $\Lambda$ é também fechado em $(0, \infty)$ para concluir que $\Lambda=(0, \infty)$. Suponha que $\left\{\lambda_{n}\right\}_{n=1}^{\infty} \subset \Lambda, \lambda_{n} \rightarrow \lambda>0$. Se $n$ é suficientemente grande temos que

$$
\left|\lambda_{n}-\lambda\right| \leq \lambda / 4
$$

Então, para n grande

$$
\left\|\left(\lambda-\lambda_{n}\right)\left(\lambda_{n}-A\right)^{-1}\right\|_{L(E)} \leq\left|\lambda_{n}-\lambda\right| \lambda_{n}^{-1} \leq 1 / 4<1
$$


e $I+\left(\lambda-\lambda_{n}\right)\left(\lambda_{n}-A\right)^{-1}$ é um isomorfismo de $E$. Logo

$$
\lambda-A=\left\{I+\left(\lambda-\lambda_{n}\right)\left(\lambda_{n}-A\right)^{-1}\right\}\left(\lambda_{n}-A\right)
$$

leva $D(A)$ sobre $E$ e $\lambda \in \rho(A)$, como queríamos.

Agora todas as hipóteses do Teorema de Hille-Yosida (ii) estão verificadas e a prova está completa.

A seguir recordamos a definição de operadores adjuntos. Seja $E$ um espaço de Banach com dual $E^{*}$. Seja $S: D(S) \subset E \rightarrow E$ um operador linear com domínio denso. O adjunto $S^{*}: D\left(S^{*}\right) \subset E^{*} \rightarrow E^{*}$ de $S$ é o operador linear definido por: $D\left(S^{*}\right)$ é o conjunto dos $e^{*} \in E^{*}$ para os quais existe $f^{*} \in E^{*}$ com

$$
\left\langle e^{*}, S e\right\rangle=\left\langle f^{*}, e\right\rangle \quad \forall e \in D(S),
$$

e se $e^{*} \in D\left(S^{*}\right)$ então $f^{*}=S^{*} e^{*}$ onde $f^{*}$ é o elemento de $E^{*}$ satisfazendo (1.6). Note que como $D(S)$ é denso em $E$ existe no máximo um $f^{*} \in E^{*}$ para o qual (1.6) vale.

Seja $H$ um espaço de Hilbert com produto escalar $\langle\cdot, \cdot\rangle$. Identificamos $H$ e $H^{*}$ e denotamos ambos por $H$.

Definição 1.6 Seja $H$ um espaço de Hilbert com produto interno $\langle\cdot, \cdot\rangle$. Um operador $A: D(A) \subset$ $H \rightarrow H$ é simétrico se $\overline{D(A)}=H$ e $A \subset A^{*}$; isto é, $\langle A e, f\rangle=\langle e, A f\rangle$ para todo e, $f \in D(A)$. $A$ é auto-adjunto se $A=A^{*}$.

Corolário 1.1 Seja A um operador linear fechado e densamente definido. Se ambos $A$ e $A^{*}$ são dissipativos, então A é o gerador infinitesimal de um semigrupo fortemente contínuo de contrações sobre E.

Prova. Pelo Teorema de Lumer-Philips é suficiente provar que $R(I-A)=E$. Como $A$ é dissipativo e fechado, $R(I-A)$ é um subespaço fechado de $E$. Suponhamos $R(I-A) \neq E$. Então existe $e^{*} \in E^{*}, e^{*} \neq 0$ tal que $\left\langle e^{*}, e-A e\right\rangle=0$ para todo $e \in D(A)$. Isto implica $e^{*}-A^{*} e^{*}=0$. Como $A^{*}$ é também dissipativo segue do Lema 1.2 que $e^{*}=0$, contradizendo a construção de $e^{*}$. Portanto, $R(I-A)=E$.

Em muitos exemplos a técnica utilizada para verificar as estimativas espectrais necessárias para se garantir que um operador $A$ é o gerador de um semigrupo fortemente contínuo, são obtidas através do conhecimento da chamada imagem numérica que é definida a seguir.

Se $A$ é um operador linear em um espaço de Banach complexo $E$, a sua imagem numérica $W(A)$ é o conjunto

$$
W(A):=\left\{\left\langle e^{*}, A e\right\rangle: e \in D(A),\|e\|_{E}=1, e^{*} \in E^{*},\left\|e^{*}\right\|_{E^{*}}=1,\left\langle e^{*}, e\right\rangle=1\right\} .
$$


No caso em que $E$ é um espaço de Hilbert, $W(A)=\left\{\langle A e, e\rangle: e \in D(A),\|e\|_{E}=1\right\}$.

Teorema 1.7 Seja $A: D(A) \subset E \rightarrow E$ um operador fechado densamente definido. Seja $W(A)$ a imagem numérica de $A$ e $\Sigma$ um subconjunto aberto e conexo em $\mathbb{C} \backslash W(A)$. Se $\lambda \notin \overline{W(A)}$ então $\lambda-A$ é um-a-um e tem imagem fechada e satisfaz

$$
\|(\lambda-A) e\|_{L(E)} \geq d(\lambda, W(A))\|e\|_{E} .
$$

Adicionalmente, se $\rho(A) \cap \Sigma \neq \varnothing$ então $\rho(A) \supset \Sigma e$

$$
\left\|(\lambda-A)^{-1}\right\|_{L(E)} \leq \frac{1}{d(\lambda, W(A))}, \quad \forall \lambda \in \Sigma
$$

onde $d(\lambda, W(A))$ é a distância de $\lambda$ a $W(A)$.

Prova. Seja $\lambda \notin \overline{W(A)}$. Se $e \in D(A),\|e\|_{E}=1, e^{*} \in E^{*},\left\|e^{*}\right\|_{E^{*}}=1$ e $\left\langle e^{*}, e\right\rangle=1$ então

$$
0<d(\lambda, W(A)) \leq\left|\lambda-\left\langle e^{*}, A e\right\rangle\right|=\left|\left\langle e^{*}, \lambda e-A e\right\rangle\right| \leq\|\lambda e-A e\|_{E} .
$$

Daí, se $0 \neq e \in D(A)$ segue de $(1.10)$ que

$$
0<d(\lambda, W(A)) \leq\left\|(\lambda-A) \frac{e}{\|e\|_{E}}\right\|_{E}
$$

e então

$$
0<d(\lambda, W(A))\|e\|_{E} \leq\|(\lambda-A) e\|_{E} .
$$

Portanto $\lambda-A$ é um-a-um, tem imagem fechada e satisfaz (1.8). Se adicionalmente $\lambda \in \rho(A)$ então (1.11) implica (1.9).

Resta mostrar que se $\Sigma$ intercepta $\rho(A)$ então $\rho(A) \supset \Sigma$. Para este fim considere o conjunto $\rho(A) \cap \Sigma$. Este conjunto é obviamente não vazio e aberto em $\Sigma$. Mas também é fechado, já que se $\lambda_{n} \in \rho(A) \cap \Sigma$ é tal que $\lambda_{n} \rightarrow \lambda \in \Sigma$ implica que para $n$ suficientemente grande $\left|\lambda-\lambda_{n}\right|<d\left(\lambda_{n}, W(A)\right)$. De (1.9) segue que para $n$ grande, $\left|\lambda-\lambda_{n}\right|\left\|\left(\lambda_{n}-A\right)^{-1}\right\|<1$ e (1.5) implica que $\lambda \in \rho(A)$ e portanto $\rho(A) \cap \Sigma$ é fechado em $\Sigma$. Segue que $\rho(A) \cap \Sigma=\Sigma$, ou seja, $\rho(A) \supset \Sigma$, como queríamos.

Definição 1.7 Dizemos que um operador $A: D(A) \subset E \longrightarrow E$ é fechável se $\overline{G r a f(A)}$ é gráfico de um operador $\bar{A}$. $\bar{A}$ é o menor operador fechado que estende $A$.

Teorema 1.8 Seja A um operador dissipativo em E.

(a) Se para algum $\lambda_{0}>0, R\left(\lambda_{0}-A\right)=E$ então $R(\lambda-A)=E$ para todo $\lambda>0$.

(b) Se A é fechável então o seu fecho $\bar{A}$ é também dissipativo. 
(c) $S e \overline{D(A)}=$ E então A é fechável.

Prova. A afirmativa (a) foi provada no Teorema de Lumer-Phillips. Para provar (b) sejam $e \in D(\bar{A}), f=\bar{A} e$. Então existe uma sequência $\left\{e_{n}\right\} \subset D(A)$ tal que $e_{n} \rightarrow e$ e $A e_{n} \rightarrow f=\bar{A} e$. Do Lema 1.2 segue que $\left\|\lambda e_{n}-A e_{n}\right\|_{E} \geq \lambda\left\|e_{n}\right\|_{E}$, para $\lambda>0$ e fazendo $n \rightarrow \infty$ temos

$$
\|\lambda e-\bar{A} e\|_{E} \geq \lambda\|e\|_{E}, \quad \lambda>0 .
$$

Como (1.12) vale para todo $e \in D(\bar{A})$, segue que $\bar{A}$ é dissipativo pelo Lema 1.2. Para provar $(c)$ admita que $A$ não é fechável. Então existe uma sequência $\left\{e_{n}\right\} \subset D(A), e_{n} \rightarrow 0$ e $A e_{n} \rightarrow f$ com $\|f\|_{E}=1$. Do Lema 1.2 segue que para todo $t>0$ e $e \in D(A)$

$$
\left\|\left(e+t^{-1} e_{n}\right)-t A\left(e+t^{-1} e_{n}\right)\right\|_{E} \geq\left\|e+t^{-1} e_{n}\right\|_{E} .
$$

Fazendo primeiro $n \rightarrow \infty$, temos

$$
\|e-t A e-f\|_{E} \geq \|\left. e\right|_{E} .
$$

e se $t \rightarrow 0$ então

$$
\|e-f\|_{E} \geq\|e\|_{E}
$$

para todo $e \in D(A)$. Mas isto é impossível se $D(A)$ é denso em $E$ e portanto $A$ é fechável.

Teorema 1.9 Seja A dissipativo com $R(I-A)=E$. Se E é reflexivo então $\overline{D(A)}=E$.

Prova. Seja $e^{*} \in E^{*}$ tal que $\left\langle e^{*}, e\right\rangle=0$ para todo $e \in D(A)$. Mostraremos que $e^{*}=0$. Como $R(I-A)=E$, é suficiente mostrar que $\left\langle e^{*}, e-A e\right\rangle=0$ para todo $e \in D(A)$, o que é equivalente a $\left\langle e^{*}, A e\right\rangle=0$ para todo $e \in D(A)$. Seja $e \in D(A)$ então, pelo Teorema 1.8 (a), existe um $e_{n}$ tal que $e=e_{n}-(1 / n) A e_{n}$. Como $A e_{n}=n\left(e_{n}-e\right) \in D(A)$, então $e_{n} \in D\left(A^{2}\right)$ e $A e=A e_{n}-(1 / n) A^{2} e_{n}$, ou seja, $A e_{n}=(I-(1 / n) A)^{-1} A e$. Do Lema 1.2 segue que $\left\|(I-(1 / n) A)^{-1}\right\|_{L(E)} \leq 1$ e portanto $\left\|A e_{n}\right\|_{E} \leq\|A e\|_{E}=C$. Também, $\left\|e_{n}-e\right\|_{E} \leq(1 / n)\left\|A e_{n}\right\|_{E} \leq(1 / n)\|A e\|_{E}$ e portanto $e_{n} \rightarrow e$. Como $\left\|A e_{n}\right\|_{E} \leq C$ e $E$ é reflexivo, existe uma subsequência $A e_{n_{k}}$ de $A e_{n}$ tal que $A e_{n_{k}} \stackrel{w}{\longrightarrow} f$ (na topologia fraca). Como $A$ é fechado (veja Teorema de Lumer-Phillips (ii)), segue que $f=A e$. Finalmente, como $\left\langle e^{*}, z\right\rangle=0$ para todo $z \in D(A)$, temos

$$
\left\langle e^{*}, A e_{n_{k}}\right\rangle=\left\langle e^{*}, n_{k}\left(e_{n_{k}}-e\right)\right\rangle=n_{k}\left\langle e^{*}, e_{n_{k}}-e\right\rangle=0 .
$$

Fazendo $n_{k} \rightarrow \infty$ em (1.13) temos $\left\langle e^{*}, A e\right\rangle=0$. Isto vale para $e \in D(A)$ e portanto $e^{*}=0$ e $\overline{D(A)}=E$.

As próximas duas seções são necessárias para alcançarmos o Teorema de Stone. 


\section{$1.4 \quad$ Fórmulas exponenciais}

Teorema 1.10 Seja $\{T(t): t \geq 0\}$ um semigrupo fortemente contínuo em $E$. Se

$$
A(h) e=\frac{T(h) e-e}{h}
$$

então para todo e $\in$ E temos

$$
T(t) e=\lim _{h \rightarrow 0^{+}} \mathbf{e}^{t A(h)} e
$$

e o limite é uniforme em $t$ em qualquer intervalo limitado de $\mathbb{R}$.

Prova. Seja $\|T(t)\|_{L(E)} \leq M \mathbf{e}^{\omega t}$ com $\omega \geq 0$ e seja $A$ o gerador infinitesimal de $\{T(t): t \geq 0\}$. Como para todo $h>0 A(h)$ é limitado, o semigrupo $\mathbf{e}^{t A(h)}$ está bem definido. Adicionalmente $A(h)$ e $T(t)$ comutam, logo o mesmo ocorre com $\mathbf{e}^{t A(h)}$ e $T(t)$. Ainda

$$
\left\|\mathbf{e}^{t A(h)}\right\|_{L(E)} \leq \mathbf{e}^{-t / h} \sum_{k=0}^{\infty}\left(\frac{t}{h}\right)^{k} \frac{\|T(h k)\|_{L(E)}}{k !} \leq M \mathbf{e}^{\frac{t}{h}\left(\mathbf{e}^{\omega h}-1\right)} .
$$

Portanto, para $0<h \leq 1$ temos

$$
\left\|\mathbf{e}^{t A(h)}\right\|_{L(E)} \leq M \mathbf{e}^{t\left(\mathbf{e}^{\omega}-1\right)} .
$$

É fácil ver que para $e \in D(A)$, a aplicação $s \mapsto \mathbf{e}^{(t-s) A(h)} T(s) e$ é diferenciável e que

$$
\begin{aligned}
\frac{d}{d s}\left(\mathbf{e}^{(t-s) A(h)} T(s) e\right) & =-A(h) \mathbf{e}^{(t-s) A(h)} T(s) e+\mathbf{e}^{(t-s) A(h)} A T(s) e= \\
& =\mathbf{e}^{(t-s) A(h)} T(s)(A e-A(h) e) .
\end{aligned}
$$

Consequentemente, para $0<h \leq 1$ e $e \in D(A)$ temos

$$
\begin{aligned}
\left\|T(t) e-\mathbf{e}^{t A(h)} e\right\|_{L(E)} & =\left\|\int_{0}^{t} \frac{d}{d s}\left(\mathbf{e}^{(t-s) A(h)} T(s) e\right) d s\right\|_{L(E)} \leq \\
& \leq \int_{0}^{t}\left\|\mathbf{e}^{(t-s) A(h)}\right\|_{L(E)}\|T(s)\|_{L(E)} d s\|A e-A(h) e\|_{E} \leq \\
& \leq t M^{2} \mathbf{e}^{t\left(\mathbf{e}^{\omega}+\omega-1\right)}\|A e-A(h) e\|_{E} .
\end{aligned}
$$

Fazendo $h \rightarrow 0^{+}$obtemos (1.14) para $e \in D(A)$. Como ambos $\left\|\mathbf{e}^{t A(h)}\right\|_{L(E)}$ e $\|T(t)\|_{L(E)}$ são uniformemente limitados em qualquer intervalo limitado de $\mathbb{R}$ e como $D(A)$ é denso em $E$ obtemos que (1.14) vale para todo $e \in E$.

Teorema 1.11 (O Segundo Limite Fundamental) Seja $\{T(t): t \geq 0\}$ um semigrupo fortemente contínuo em E. Se A é o seu gerador infinitesimal, então

$$
T(t) e=\lim _{n \rightarrow \infty}\left(I-\frac{t}{n} A\right)^{-n} e=\lim _{n \rightarrow \infty}\left[\frac{n}{t}\left(\frac{n}{t}-A\right)^{-1}\right]^{n} e, \quad \forall e \in E
$$

e os limites são uniformes para $t$ em intervalos limitados de $\mathbb{R}$. 
Prova. Admita que $\|T(t)\|_{L(E)} \leq M \mathbf{e}^{\omega t}$. Vimos no Teorema 1.2 que para $\operatorname{Re} \lambda>\omega,(\lambda-A)^{-1}$ é analítica em $\lambda$ e que

$$
(\lambda-A)^{-1} e=\int_{0}^{\infty} \mathbf{e}^{-\lambda s} T(s) e d s, \quad e \in E .
$$

Para $t$ fixo e $n$ suficientemente grande $(n / t>\omega)$, derivando $n$ vezes em $\lambda$, substituindo $s=v t$ e tomando $\lambda=n / t$ em (1.15) encontramos

$$
\left[\left(\frac{n}{t}-A\right)^{-1}\right]^{(n)} e=(-1)^{n} t^{n+1} \int_{0}^{\infty}\left(v \mathbf{e}^{-v}\right)^{n} T(t v) e d v
$$

Mas

$$
\left[(\lambda-A)^{-1}\right]^{(n)}=(-1)^{n} n !(\lambda-A)^{-n-1}
$$

e portanto

$$
\left[\frac{n}{t}\left(\frac{n}{t}-A\right)^{-1}\right]^{n+1} e=\frac{n^{n+1}}{n !} \int_{0}^{\infty}\left(v \mathbf{e}^{-v}\right)^{n} T(t v) e d v
$$

Notando que

$$
\frac{n+1}{n !} \int_{0}^{\infty}\left(v \mathbf{e}^{-v}\right)^{n} d v=1
$$

obtemos

$$
\left[\frac{n}{t}\left(\frac{n}{t}-A\right)^{-1}\right]^{n+1} e-T(t) e=\frac{n^{n+1}}{n !} \int_{0}^{\infty}\left(v \mathbf{e}^{-v}\right)^{n}[T(t v) e-T(t) e] d v .
$$

Dado $\epsilon>0$ escolhemos $0<a<1<b<\infty$ tal que $t \in\left[0, t_{0}\right]$ implica

$$
\|T(t v) e-T(t) e\|_{L(E)}<\epsilon, \quad a \leq v \leq b .
$$

Então quebramos a integral em três integrais $I_{1}, I_{2}, I_{3}$ nos intervalos $[0, a],[a, b]$ e $[b, \infty)$ respectivamente e temos

$$
\begin{gathered}
\left\|I_{1}\right\|_{L(E)} \leq \frac{n^{n+1}}{n !}\left(a \mathbf{e}^{-a}\right)^{n} \int_{0}^{a}\|T(t v) e-T(t) e\|_{L(E)} d v, \\
\left\|I_{2}\right\|_{L(E)} \leq \epsilon \frac{n^{n+1}}{n !} \int_{a}^{b}\left(v \mathbf{e}^{-v}\right)^{n} d v<\epsilon, \\
\left\|I_{3}\right\|_{L(E)}=\left\|\frac{n^{n+1}}{n !} \int_{b}^{\infty}\left(v \mathbf{e}^{-v}\right)^{n}(T(t v) e-T(t) e) d v\right\|_{L(E) .}
\end{gathered}
$$

Aqui usamos o fato que $v \mathbf{e}^{-v} \geq 0$ é não decrescente para $0 \leq v \leq 1$ e não crescente para $v \geq 1$. Como adicionalmente $v \mathbf{e}^{-v}<\mathbf{e}^{-1}$ para $v \neq 1,\left\|I_{1}\right\|_{L(E)} \rightarrow 0$ uniformemente para $t \in\left[0, t_{0}\right]$ quando $n \rightarrow \infty$. Escolhendo $n>\omega t$ em $I_{3}$, vemos que a integral na estimativa de $I_{3}$, converge e que $\left\|I_{3}\right\|_{L(E)} \rightarrow 0$ uniformemente para $t \in\left[0, t_{0}\right]$ quando $n \rightarrow \infty$. Consequentemente

$$
\limsup _{n \rightarrow \infty}\left\|\left[\frac{n}{t}\left(\frac{n}{t}-A\right)^{-1}\right]^{n+1} e-T(t) e\right\|_{L(E)} \leq \epsilon
$$


e como $\epsilon>0$ é arbitrário temos

$$
\lim _{n \rightarrow \infty}\left[\frac{n}{t}\left(\frac{n}{t}-A\right)^{-1}\right]^{n+1} e=T(t) e .
$$

Ainda

$$
\lim _{n \rightarrow \infty} \frac{n}{t}\left(\frac{n}{t}-A\right)^{-1} e=e
$$

e o resultado segue.

\subsection{Pseudo-resolventes}

Seja $A$ um operador fechado e densamente definido em $E$. Se $\mu$ e $\lambda$ estão em $\rho(A)$, então temos o que chamamos de identidade do resolvente

$$
(\lambda I-A)^{-1}-(\mu I-A)^{-1}=(\mu-\lambda)(\lambda I-A)^{-1}(\mu I-A)^{-1} .
$$

De fato,

$$
\begin{aligned}
(\mu-\lambda)(\lambda I-A)^{-1}(\mu I-A)^{-1} & =\mu(\lambda I-A)^{-1}(\mu I-A)^{-1}-\lambda(\lambda I-A)^{-1}(\mu I-A)^{-1}= \\
& =\left[\mu(\lambda I-A)^{-1}-\lambda(\lambda I-A)^{-1}\right](\mu I-A)^{-1}= \\
& =\left[\mu(\lambda I-A)^{-1}-\left[I+A(\lambda I-A)^{-1}\right]\right](\mu I-A)^{-1}= \\
& =\left[-I+(\lambda I-A)^{-1}(\mu I-A)\right](\mu I-A)^{-1}= \\
& =(\lambda I-A)^{-1}-(\mu I-A)^{-1} .
\end{aligned}
$$

Motivado por isto definimos

Definição 1.8 Seja $\Delta$ um subconjunto do plano complexo. Uma família $\{J(\lambda), \lambda \in \Delta\}$, de operadores lineares limitados em E satisfazendo

$$
J(\lambda)-J(\mu)=(\mu-\lambda) J(\lambda) J(\mu), \quad \lambda, \mu \in \Delta
$$

é chamado um pseudo-resolvente em $\Delta$.

O objetivo final desta seção é determinar condições sob as quais existe um operador fechado e densamente definido $A$ tal que $J(\lambda)$ é o resolvente de $A$.

Lema 1.3 Seja $\Delta$ um subconjunto de $\mathbb{C}$. Se $J(\lambda)$ é pseudo-resolvente em $\Delta$, então $J(\lambda) J(\mu)=$ $J(\mu) J(\lambda)$. O núcleo $N(J(\lambda))$ e a imagem $R(J(\lambda))$ são independentes de $\lambda \in \Delta . N(J(\lambda))$ é um subespaço fechado de E. 
Prova. Por (1.17) segue que $J(\lambda)$ e $J(\mu)$ comutam para $\lambda, \mu \in \Delta$. Vejamos, se $\lambda \neq \mu$,

$$
J(\lambda) J(\mu)=\frac{J(\lambda)-J(\mu)}{\lambda-\mu}=\frac{J(\mu)-J(\lambda)}{\mu-\lambda}=J(\mu) J(\lambda) .
$$

Também, reescrevendo (1.17) na forma

$$
J(\lambda)=J(\mu)[I+(\mu-\lambda) J(\lambda)]
$$

obtemos que $R(J(\mu)) \supset R(J(\lambda))$ e por simetria temos a igualdade. Semelhantemente $N(J(\lambda))=$ $N(J(\mu))$. Como $J(\lambda)$ é um operador linear limitado para cada $\lambda \in \Delta$, segue que $N(J(\lambda)) \subset E$ é um subespaço fechado.

Teorema 1.12 Seja $\Delta$ um subconjunto de $\mathbb{C}$ e seja $J(\lambda)$ pseudo-resolvente em $\Delta$. Então, $J(\lambda)$ é o resolvente de um operador (unicamente definido) linear fechado e densamente definido se, $e$ somente se, $N(J(\lambda))=\{0\}$ e $R(J(\lambda))$ é denso em $E$.

Prova. Se $J(\lambda)$ é o resolvente de um operador fechado e densamente definido $A: D(A) \subset E \rightarrow E$, então $J(\lambda)=(\lambda I-A)^{-1}: R(\lambda I-A) \rightarrow D(A)$. Logo, $N(J(\lambda))=\{0\}$ e $R(J(\lambda))=D(A)$ é denso em $E$. Por outro lado, admita que $N(J(\lambda))=\{0\}$ e $R(J(\lambda))$ é denso em $E$. De $N(J(\lambda))=\{0\}$ segue que $J(\lambda)$ é um-a-um. Seja $\lambda_{0} \in \Delta$ e defina

$$
A=\lambda_{0} I-J\left(\lambda_{0}\right)^{-1}: R\left(J\left(\lambda_{0}\right)\right) \longrightarrow E .
$$

Note que $A$ está bem definido, pois para cada $e \in R(J(\lambda)), \lambda, \mu \in \Delta$

$$
J(\lambda) J(\mu)\left[J(\mu)^{-1} e-J(\lambda)^{-1} e\right]=J(\lambda) e-J(\mu) e=(\mu-\lambda) J(\lambda) J(\mu) e
$$

assim,

$$
J(\mu)^{-1} e-J(\lambda)^{-1} e=(\mu-\lambda) e
$$

e portanto,

$$
\lambda e-J(\lambda)^{-1} e=\mu e-J(\mu)^{-1} e,
$$

ou seja, $\lambda_{0}-J\left(\lambda_{0}\right)^{-1}$ é independente de $\lambda_{0}$. Observe que $A$ assim definido é claramente linear, fechado, pois $J\left(\lambda_{0}\right)$ é linear limitado, e ainda, $A$ é densamente definido, já que $D(A)=$ $D\left(J\left(\lambda_{0}^{-1}\right)\right)=R\left(J\left(\lambda_{0}\right)\right)$. Da definição de $A$, é claro que

$$
\left(\lambda_{0} I-A\right) J\left(\lambda_{0}\right)=I=J\left(\lambda_{0}\right)\left(\lambda_{0} I-A\right),
$$

e portanto $J\left(\lambda_{0}\right)=\left(\lambda_{0} I-A\right)^{-1}$. Se $\lambda \in \Delta$, então

$$
\begin{aligned}
(\lambda I-A) J(\lambda) & =\left(\left(\lambda-\lambda_{0}\right) I+\left(\lambda_{0} I-A\right)\right) J(\lambda)= \\
& =\left(\left(\lambda-\lambda_{0}\right) I+\left(\lambda_{0} I-A\right)\right) J\left(\lambda_{0}\right)\left[I-\left(\lambda-\lambda_{0}\right) J(\lambda)\right]= \\
& =I+\left(\lambda-\lambda_{0}\right)\left[J\left(\lambda_{0}\right)-J(\lambda)-\left(\lambda-\lambda_{0}\right) J\left(\lambda_{0}\right) J(\lambda)\right]= \\
& =I
\end{aligned}
$$


e semelhantemente $J(\lambda)(\lambda I-A)=I$. Portanto, $J(\lambda)=(\lambda I-A)^{-1}$ para todo $\lambda \in \Delta$. Em particular, $A$ é unicamente determinado por $J(\lambda)$.

A seguir damos condições suficientes para que pseudo-resolventes sejam resolventes.

Teorema 1.13 Seja $\Delta \subset \mathbb{C}$ ilimitado e seja $J(\lambda)$ um pseudo-resolvente em $\Delta$. Se $R(J(\lambda))$ é denso em $E$ e existe uma sequência $\lambda_{n} \in \Delta$ com $\left|\lambda_{n}\right| \rightarrow \infty e$

$$
\left\|\lambda_{n} J\left(\lambda_{n}\right)\right\|_{L(E)} \leq M
$$

para alguma constante $M$, então $J(\lambda)$ é o resolvente de um único operador fechado e densamente definido.

Prova. Como $\left|\lambda_{n}\right| \rightarrow \infty$, segue de (1.18) que $\left\|J\left(\lambda_{n}\right)\right\|_{L(E)} \rightarrow 0$ quando $n \rightarrow \infty$. Seja $\mu \in \Delta$. De (1.17) deduzimos que

$$
\left\|\left(\lambda_{n} J\left(\lambda_{n}\right)-I\right) J(\mu)\right\|_{L(E)}=\left\|(\mu J(\mu)-I) J\left(\lambda_{n}\right)\right\|_{L(E)} \rightarrow 0, \quad n \rightarrow \infty .
$$

Portanto, se $e \in R(J(\mu))$ temos

$$
\lambda_{n} J\left(\lambda_{n}\right) e \rightarrow e, \quad n \rightarrow \infty
$$

Como $R(J(\mu))$ é denso em $E$ e $\lambda_{n} J\left(\lambda_{n}\right)$ é uniformemente limitada, temos que (1.19) vale para todo $e \in E$. Agora, se $e \in N(J(\lambda))$, então $\lambda_{n} J\left(\lambda_{n}\right) e=0$ e de (1.19) deduzimos que $e=0$. Portanto $N(J(\lambda))=\{0\}$ e, do Teorema 1.12, $J(\lambda)$ é o resolvente de um único operador fechado e densamente definido $A$.

Corolário 1.2 Seja $\Delta \subset \mathbb{C}$ ilimitado e $J(\lambda)$ um pseudo-resolvente em $\Delta$. Se existe uma seqüência $\lambda_{n} \in \Delta$ tal que $\left|\lambda_{n}\right| \rightarrow \infty$ quando $n \rightarrow \infty e$

$$
\lim _{n \rightarrow \infty} \lambda_{n} J\left(\lambda_{n}\right) e=e, \quad \forall e \in E
$$

então $J(\lambda)$ é o resolvente de um operador (unicamente definido) fechado e densamente definido $A$.

Prova. Do Princípio da Limitação Uniforme e de (1.20) seque que (1.18) vale. Do Lema 1.3 sabemos que $R(J(\lambda))$ é independente de $\lambda \in \Delta$ e portanto (1.20) implica que $R(J(\lambda))$ é denso em E. Portanto, as condições do Teorema 1.13 estão satisfeitas e o resultado segue. 


\subsection{O semigrupo dual e o Teorema de Stone}

Começamos com alguns resultados básicos sobre operadores duais.

Lema 1.4 Seja $S \in L(E)$; então, $S^{*} \in L\left(E^{*}\right)$ e $\|S\|_{L(E)}=\left\|S^{*}\right\|_{L(E)}$.

Prova. É fácil ver que $S^{*}$ é operador linear fechado. Além disso, para todo $e^{*} \in E^{*},\left\langle e^{*}, S e\right\rangle$ é um funcional linear contínuo e portanto determina um único elemento $f^{*} \in E^{*}$ para o qual $\left\langle f^{*}, e\right\rangle=\left\langle e^{*}, S e\right\rangle$. Logo, $D\left(S^{*}\right)=E^{*}$. Segue então do Teorema do Gráfico Fechado que $S^{*} \in L\left(E^{*}\right)$. Adicionalmente,

$$
\begin{aligned}
\left\|S^{*}\right\|_{L\left(E^{*}\right)} & =\sup _{\left\{\left\|e^{*}\right\|_{E^{*}} \leq 1\right\}}\left\|S^{*} e^{*}\right\|_{E^{*}}=\sup _{\left\{\left\|e^{*}\right\|_{E^{*}} \leq 1\right\}} \sup _{\left\{\|e\|_{E} \leq 1\right\}}\left|\left\langle S^{*} e^{*}, e\right\rangle\right|= \\
& =\sup _{\{\|e\| \leq 1\}} \sup _{\left\{\left\|e^{*}\right\|_{E^{*}} \leq 1\right\}}\left|\left\langle e^{*}, S e\right\rangle\right|=\sup _{\left\{\|e\|_{E} \leq 1\right\}}\|S e\|_{E}= \\
& =\|S\|_{L(E)},
\end{aligned}
$$

e o resultado segue.

Lema 1.5 Seja $A$ um operador linear densamente definido em $E$. Se $\lambda \in \rho(A)$ então $\lambda \in \rho\left(A^{*}\right)$ $e$

$$
\left(\lambda I-A^{*}\right)^{-1}=\left((\lambda I-A)^{-1}\right)^{*} .
$$

Prova. Da definição de adjunto temos $(\lambda I-A)^{*}=\lambda I^{*}-A^{*}$. Como $(\lambda I-A)^{-1} \in L(E)$ segue do Lema 1.4 que $\left((\lambda I-A)^{-1}\right)^{*} \in L\left(E^{*}\right)$. Resta mostrar que $\left(\lambda I^{*}-A^{*}\right)^{-1}$ existe e é igual a $\left((\lambda I-A)^{-1}\right)^{*}$. Primeiramente mostremos que $\lambda I^{*}-A^{*}$ é injetor. Se para algum $e^{*} \neq 0$, $e^{*} \in D\left(A^{*}\right)$, tivermos $\left(\lambda I^{*}-A^{*}\right) e^{*}=0$, então $0=\left\langle\left(\lambda I^{*}-A^{*}\right) e^{*}, e\right\rangle=\left\langle e^{*},(\lambda I-A) e\right\rangle$ para todo $e \in D(A)$. Mas como $\lambda \in \rho(A), R(\lambda I-A)=E$ e portanto $e^{*}=0$, contradizendo a escolha de $e^{*}$. Portanto, $\lambda I^{*}-A^{*}$ é injetor. Se agora $e \in E, e^{*} \in D\left(A^{*}\right)$, então

$$
\left\langle e^{*}, e\right\rangle=\left\langle e^{*},(\lambda I-A)(\lambda I-A)^{-1} e\right\rangle=\left\langle\left(\lambda I^{*}-A^{*}\right) e^{*},(\lambda I-A)^{-1} e\right\rangle=\left\langle\left((\lambda I-A)^{-1}\right)^{*}\left(\lambda I^{*}-A^{*}\right) e^{*}, e\right\rangle
$$

e portanto

$$
\left((\lambda I-A)^{-1}\right)^{*}\left(\lambda I^{*}-A^{*}\right) e^{*}=e^{*}, \quad \forall e^{*} \in D\left(A^{*}\right) .
$$

Por outro lado se $e^{*} \in E^{*}$ e $e \in D(A)$ então

$$
\left\langle e^{*}, e\right\rangle=\left\langle e^{*},(\lambda I-A)^{-1}(\lambda I-A) e\right\rangle=\left\langle\left((\lambda I-A)^{-1}\right)^{*} e^{*},(\lambda I-A) e\right\rangle=\left\langle(\lambda I-A)^{*}\left((\lambda I-A)^{-1}\right)^{*} e^{*}, e\right\rangle
$$

o que implica que

$$
\left(\lambda I^{*}-A^{*}\right)\left((\lambda I-A)^{-1}\right)^{*} e^{*}=e^{*}, \quad \forall e^{*} \in E^{*} .
$$

Segue que $\lambda \in \rho\left(A^{*}\right)$ e que $\left(\lambda I^{*}-A^{*}\right)^{-1}=\left((\lambda I-A)^{-1}\right)^{*}$. 
Seja $\{T(t): t \geq 0\}$ um semigrupo fortemente contínuo em $E$. Para $t>0$ seja $\left\{T(t)^{*}: t \geq 0\right\}$ o semigrupo dual. O semigrupo dual não precisa ser fortemente contínuo em $E^{*}$.

Definição 1.9 Seja $S$ um operador linear em $E$ e seja $F$ um subespaço de E. O operador $\tilde{S}$ definido por $D(\tilde{S})=\{e \in D(S) \cap F: S e \in F\}$ e $\tilde{S} e=S e$ para $e \in D(\tilde{S})$ é chamado parte de $S$ em F.

Teorema 1.14 Seja $\{T(t): t \geq 0\}$ um semigrupo fortemente contínuo em $E$ com gerador infinitesimal $A$ e $\left\{T(t)^{*}: t \geq 0\right\}$ o semigrupo dual. Se $A^{*}$ é o adjunto de $A$ e $E^{\odot}$ é o fecho de $D\left(A^{*}\right)$ em $E^{*}$, então a restrição $\left\{T(t)^{\odot}: t \geq 0\right\}$ de $\left\{T(t)^{*}: t \geq 0\right\}$ a $E^{\odot}$ é um semigrupo fortemente contínuo em $E^{\odot}$. O gerador infinitesimal $A^{\odot}$ de $\left\{T(t)^{\odot}: t \geq 0\right\}$ é a parte de $A^{*}$ em $E^{\odot}$.

Prova. Como $A$ é o gerador infinitesimal de $\{T(t): t \geq 0\}$, da Forma Geral do Teorema de Hille-Yosida, existem constantes $\omega$ e $M$ tais que para todo $\lambda>\omega$, temos $\lambda \in \rho(A)$ e

$$
\left\|(\lambda-A)^{-n}\right\|_{L(E)} \leq \frac{M}{(\lambda-\omega)^{n}}, \quad n=1,2, \cdots
$$

Segue então do Lema 1.5 que $\lambda \in \rho\left(A^{*}\right)$ e do Lema 1.4 que

$$
\left\|\left(\lambda I^{*}-A^{*}\right)^{-n}\right\|_{L\left(E^{*}\right)} \leq \frac{M}{(\lambda-\omega)^{n}}, \quad n=1,2, \cdots
$$

Seja $J(\lambda)$ a restrição de $\left(\lambda I^{*}-A^{*}\right)^{-1}$ a $E^{\odot}$. Então

$$
\left\|J(\lambda)^{n}\right\|_{L(E \odot)} \leq \frac{M}{(\lambda-\omega)^{n}}, n=1,2, \cdots
$$

Observe que $A^{*}$ é fechado e $E^{\odot}=\overline{D\left(A^{*}\right)}$ em $E^{*}$ e então da identidade do resolvente,

$$
J(\lambda)-J(\mu)=(\mu-\lambda) J(\lambda) J(\mu), \quad \lambda, \mu>\omega
$$

e $J(\lambda)$ é um pseudo-resolvente em $(0, \infty) \subset \mathbb{C}$. Exatamente como na prova do Teorema de Hille-Yosida, temos que

$$
\lambda J(\lambda)=\left(I^{*}-\lambda^{-1} A^{*}\right)^{-1}=I^{*}+A^{*} J(\lambda) .
$$

Para $e \in D\left(A^{*}\right)$, segue que

$$
\begin{aligned}
\left\|\lambda J(\lambda) e^{*}-e^{*}\right\|_{E \odot} & =\left\|A^{*} J(\lambda) e^{*}\right\|_{E \odot}=\|J(\lambda)\|_{L(E \odot)}\left\|A^{*} e^{*}\right\|_{E \odot} \leq \\
& \leq \frac{M}{(\lambda-\omega)}\left\|A^{*} e^{*}\right\|_{E \odot} \stackrel{\lambda \rightarrow \infty}{\longrightarrow} 0 .
\end{aligned}
$$


Como $\overline{D\left(A^{*}\right)}=E^{\odot}$, segue que

$$
\lim _{\lambda \rightarrow \infty} \lambda J(\lambda) e^{*}=e^{*}, \quad \forall e^{*} \in E^{\odot} .
$$

Segue do Corolário 1.2 que $J(\lambda)$ é o resolvente de um operador fechado e densamente definido $A^{\odot}$ em $E^{\odot}$. Ainda, segue de $(1.21)$ e da Forma Geral do Teorema de Hille-Yosida, que $A^{\odot}$ é o gerador infinitesimal de um semigrupo fortemente contínuo $\left\{T(t)^{\odot}: t \geq 0\right\}$ em $E^{\odot}$.

Vamos mostrar que $\left\{T(t)^{\odot}: t \geq 0\right\}$ é a restrição de $\left\{T(t)^{*}: t \geq 0\right\}$ a $E^{\odot}$. Para $e \in E$ e $e^{\odot} \in E^{\odot}$ temos

$$
\left\langle e^{\odot},\left(I-\frac{t}{n} A\right)^{-n} e\right\rangle=\left\langle\left(I^{\odot}-\frac{t}{n} A^{\odot}\right)^{-n} e^{\odot}, e\right\rangle, \quad n=1,2,3 \cdots
$$

Fazendo $n \rightarrow \infty$ e usando o Teorema 1.11 , obtemos

$$
\left\langle e^{\odot}, T(t) e\right\rangle=\left\langle T(t)^{\odot} e^{\odot}, e\right\rangle .
$$

Segue que para $e^{\odot} \in E^{\odot}, T(t)^{*} e^{\odot}=T(t)^{\odot} e^{\odot}$ e assim $T(t)^{\odot}$ é a restrição de $T(t)^{*}$ a $E^{\odot}$.

Para concluir a prova temos que mostrar que $A^{\odot}$ é a parte de $A^{*}$ em $E^{\odot}$. Seja $e^{*} \in D\left(A^{*}\right)$ tal que $e^{*} \in E^{\odot}$ e $A^{*} e^{*} \in E^{\odot}$. Então $\left(\lambda I^{*}-A^{*}\right) e^{*} \in E^{\odot} \mathrm{e}$

$$
\left(\lambda I^{\odot}-A^{\odot}\right)^{-1}\left(\lambda I^{*}-A^{*}\right) e^{*}=e^{*} .
$$

Portanto $e^{*} \in D\left(A^{\odot}\right)$ e aplicando $\lambda I^{\odot}-A^{\odot}$ em ambos os lados da igualdade acima temos $\left(\lambda I^{*}-A^{*}\right) e^{*}=\left(\lambda I^{\odot}-A^{\odot}\right) e^{*}$ e portanto $A^{\odot} e^{*}=A^{*} e^{*}$. Isto mostra que $A^{\odot}$ é a parte de $A^{*}$ em $E^{*}$.

O seguinte resultado identifica alguns casos em que o semigrupo dual é fortemente contínuo.

Lema 1.6 Seja $E$ um espaço de Banach real reflexivo. Se $S: D(S) \subset E \rightarrow E$ é fechado e densamente definido então $D\left(S^{*}\right)$ é denso em $E^{*}$.

Prova. Se $D\left(S^{*}\right)$ não é denso em $E^{*}$, então existe um elemento $e_{0} \in E$ tal que $e_{0} \neq 0$ e $\left\langle e^{*}, e_{0}\right\rangle=0$, para todo $e^{*} \in D\left(S^{*}\right)$. Como $S$ é fechado seu gráfico é fechado e não contém $\left(0, e_{0}\right)$. Da Forma Geométrica do Teorema de Hahn-Banach existem, $e_{1}^{*}$ e $e_{2}^{*}$ em $E^{*}$ e $\alpha \in \mathbb{R}$ tais que

$$
\left\langle e_{1}^{*}, e\right\rangle+\left\langle e_{2}^{*}, S e\right\rangle<\alpha<\left\langle e_{2}^{*}, e_{0}\right\rangle, \quad \forall e \in D(S)
$$

e então

$$
\left\langle e_{1}^{*}, 0\right\rangle+\left\langle e_{2}^{*}, S e\right\rangle=0, \quad \forall e \in D(S)
$$

e

$$
\left\langle e_{2}^{*}, e_{0}\right\rangle>0 \text {. }
$$

Segue de (1.22) que $e_{2}^{*} \in D\left(S^{*}\right)$, com $S^{*} e_{2}^{*}=-e_{1}^{*}$ e então $\left\langle e_{2}^{*}, e_{0}\right\rangle=0$, o que contradiz (1.23). Portanto $D\left(S^{*}\right)$ é denso em $E^{*}$. 
Corolário 1.3 Seja E um espaço de Banach reflexivo e $\{T(t): t \geq 0\}$ um semigrupo fortemente contínuo em $E$ com gerador infinitesimal $A$. O semigrupo dual $\left\{T(t)^{*}: t \geq 0\right\}$ de $\{T(t): t \geq 0\}$ é um semigrupo fortemente contínuo em $E^{*}$ cujo gerador infinitesimal é $A^{*}$.

Prova. Pelo Lema 1.6, $\overline{D\left(A^{*}\right)}=E^{*}$. Daí, tome $E^{\odot}=E^{*}$ no Teorema 1.14, e o resultado segue.

Uma vez que a restrição de $T(t)^{*}$ ao subespaço $E^{\odot}$ é um semigrupo fortemente contínuo, estamos exatamente na mesma posição que começamos. Em um espaço de Banach $E^{\odot}$ e com um semigrupo fortemente contínuo $\left\{T(t)^{\odot}: t \geq 0\right\}$ gerado pela parte $A^{\odot}$ de $A^{*}$ em $E^{\odot}$.

Podemos introduzir o espaço $E^{\odot *}$ e o semigrupo dual $T(t)^{\odot *}$ que é fortemente contínuo em $E^{\odot \odot}:=\overline{D\left(A^{\odot *}\right)}$.

A dualidade entre os elementos de $E$ e $E^{\odot}$ pode ser usada para definir uma imersão $j$ (note que $E^{\odot}$ é fraco-* denso em $\left.E^{*}\right)$ de $E$ em $E^{\odot *}$ com

$$
\left\langle j e, e^{\odot}\right\rangle_{E^{\odot *}, E} \odot=\left\langle e^{\odot}, e\right\rangle_{E^{\odot}, E} \cdot
$$

É claro que

$$
T(t)^{\odot *} j e=j(T(t) e)
$$

e portanto $j(E) \subset E^{\odot \odot}$. Sempre que $j(E)=E^{\odot \odot}$ chamaremos $E$ de $\odot$-reflexivo com respeito ao semigrupo $\{T(t): t \geq 0\}$.

Seja $H$ um espaço de Hilbert. Um operador limitado $U$ é unitário se $U^{*}=U^{-1}$. Recorde que $U$ é unitário se e somente se $R(U)=H$ e $U$ é uma isometria. De fato, se $U^{*} U=I$, então $e_{1}, e_{2} \in H$,

$$
\left\langle U e_{1}, U e_{2}\right\rangle=\left\langle U^{*} U e_{1}, e_{2}\right\rangle=\left\langle e_{1}, e_{2}\right\rangle .
$$

Tomando $e_{1}=e_{2}$ segue que $U$ é uma isometria e $R(U)=H$. Por outro lado, se $R(U)=H$ e $U$ é uma isometria, então $U$ é inversível e

$$
\left\langle U^{*} U e, e\right\rangle=\langle U e, U e\rangle=\|U e\|^{2}=\|e\|^{2}=\langle e, e\rangle, \quad \forall e \in H .
$$

Portanto, $U^{*} U=\mathrm{I}$.

Definição 1.10 Um grupo de operadores lineares em $E$ é uma família $\{T(t): t \in \mathbb{R}\} \subset L(E)$ tal que

(i) $T(0)=I_{E}$,

(ii) $T(t+s)=T(t) T(s)$, para todo $t, s \in \mathbb{R}$.

Se adicionalmente 
(iii) $\|T(t) e-e\|_{E} \rightarrow 0$ quando $t \rightarrow 0, \forall e \in E$, dizemos que o grupo é fortemente contínuo.

Teorema 1.15 (Stone) Um operador $A$ é o gerador infinitesimal de um grupo fortemente contínuo de operadores unitários em um espaço de Hilbert $H$ se, e somente se, iA é auto-adjunto.

Prova. Se $A$ é o gerador de um grupo fortemente contínuo de operadores unitários $\{U(t): t \in \mathbb{R}\}$, então $A$ é densamente definido e utilizando o Corolário 1.3 obtemos, para $x \in D(A)$,

$$
-A x=\lim _{t \rightarrow 0^{+}} \frac{U(-t) x-x}{t}=\lim _{t \rightarrow 0^{+}} \frac{U^{*}(t) x-x}{t}=A^{*} x
$$

o que implica $A=-A^{*}$ e portanto $(i A)^{*}=i A$ e $i A$ é auto-adjunto.

Se por outro lado $i A$ é auto adjunto então $A$ é densamente definido e $A=-A^{*}$. Portanto, para todo $x \in D(A)$ temos

$$
\langle A x, x\rangle=\left\langle x, A^{*} x\right\rangle=-\overline{\langle A x, x\rangle}
$$

e portanto $\operatorname{Re}\langle A x, x\rangle=0$ para todo $x \in D(A)$, isto é, $A$ é dissipativo. Além disso, como $A=-A^{*}$, então $\operatorname{Re}\left\langle A^{*} x, x\right\rangle=0$ para todo $x \in D(A)=D\left(A^{*}\right)$ e assim $A^{*}$ também é dissipativo. Logo $A$ e $A^{*}$ são densamente definidos e fechados e como $A^{* *}=A$, do Corolário 1.1, ambos $A$ e $A^{*}=-A$ são geradores infinitesimais de semigrupos fortemente contínuos de contrações em $H$. Se $\left\{U_{+}(t): t \geq 0\right\}$ e $\left\{U_{-}(t): t \geq 0\right\}$ são os semigrupos de contrações gerados por $A$ e $A^{*}$ respectivamente, definimos

$$
U(t)= \begin{cases}U_{+}(t), & t \geq 0 \\ U_{-}(-t), & t \leq 0\end{cases}
$$

Então $U(t)$ é um grupo. De fato, como $A$ e $-A$ são geradores de semigrupos fortemente contínuos $U_{+}(t)$ e $U_{-}(-t)$ que comutam, se $W(t)=U_{+}(t) U_{-}(t), t \geq 0$, então para $x \in D(A)=D(-A)$,

$$
\frac{W(t) x-x}{t}=U_{-}(t) \frac{U_{+}(t) x-x}{t}+\frac{U_{-}(t) x-x}{t} \rightarrow A x-A x=0, \quad \text { quando } t \rightarrow 0^{+} .
$$

Assim, se $G(t)=W(t) x, t \geq 0$, temos $G(0)=x$ e $G^{\prime}(0)=0$. Além disso,

$\frac{G(t+h)-G(t)}{h}=\frac{W(t+h) x-W(t) x}{h}=W(t) \frac{W(h) x-x}{h} \rightarrow W(t) G^{\prime}(0)=0 \quad$ quando $h \rightarrow 0^{+}$.

Logo, $G^{\prime}(t)=0$, para todo $t \geq 0$ e $G$ é constante. Daí, como $G(0)=x$ para cada $x \in D(A)$, temos que $W(t) x=x, \forall x \in D(A), t \geq 0$. Como $D(A)$ é denso em $H$ e $W(t)$ é limitado temos que $W(t)=I$, ou seja, $U_{-}(t)=\left(U_{+}(t)\right)^{-1}$. Com isto, obtemos

i) $\mathrm{U}(0)=\mathrm{I}$. 
ii) $U(t+s)=U(t) U(s),-\infty<t, s<\infty$. De fato, se $t, s>0$ ou $t, s<0$ a igualdade segue, já que $\left\{U_{+}(t): t \geq 0\right\}$ e $\left\{U_{-}(t): t \geq 0\right\}$ são semigrupos. Seja $s<0<t$. Se $t+s \geq 0$, então

$$
U(t+s) U(-s)=U_{+}(t+s) U_{+}(-s)=U_{+}(t)
$$

e assim

$$
U(t+s)=U_{+}(t) U_{+}(-s)^{-1}=U_{+}(t) U_{-}(-s)=U(t) U(s) .
$$

Por outro lado, se $t+s \leq 0$, então

$$
U(-t) U(t+s)=U_{-}(t) U_{-}(-t-s)=U_{-}(-s)
$$

e assim

$$
U(t+s)=\left(U_{-}(t)\right)^{-1} U_{-}(-s)=U_{+}(t) U_{-}(-s)=U(t) U(s)
$$

Mostramos assim que $\{U(t): t \in \mathbb{R}\}$ é grupo. Além disso, como $\left\{U_{+}(t): t \geq 0\right\}$ e $\left\{U_{-}(t): t \geq 0\right\}$ são semigrupos fortemente contínuos, segue que $\{U(t): t \in \mathbb{R}\}$ é um grupo fortemente contínuo.

Finalmente, observe que como $U(t)^{-1}=U(-t),\|U(t)\| \leq 1,\|U(-t)\| \leq 1$, segue que $R(U(t))=H$ e $U(t)$ é uma isometria para todo $t$ e portanto $U(t)$ é um grupo unitário de operadores sobre $H$, como queríamos.

\subsection{Transformada inversa de Laplace}

Vimos no Teorema $1.2(5)$ que

$$
(\lambda-A)^{-1}=\int_{0}^{\infty} \mathbf{e}^{-\lambda t} T(t) d t
$$

se $\operatorname{Re} \lambda$ é grande. Isto sugere que usando a transformada inversa de Laplace poderemos encontrar $T(t)$, conhecido $A$. No que segue perseguimos este objetivo.

\section{Lema 1.7}

(a) $\int_{-\infty}^{\infty} \frac{\operatorname{sen} t}{t} d t=\pi$

(b) Se $f: \mathbb{R} \rightarrow \mathbb{C}$ é tal que $f(t) /(1+|t|)$ é integrável em $\mathbb{R} e \int_{-1}^{1}\left|\frac{f(t)-f(0)}{t}\right| d t<\infty$, então

$$
\int_{-\infty}^{\infty} f(t) \frac{\operatorname{sen} N t}{\pi t} d t \rightarrow f(0) \quad \text { quando } \quad N \rightarrow+\infty
$$




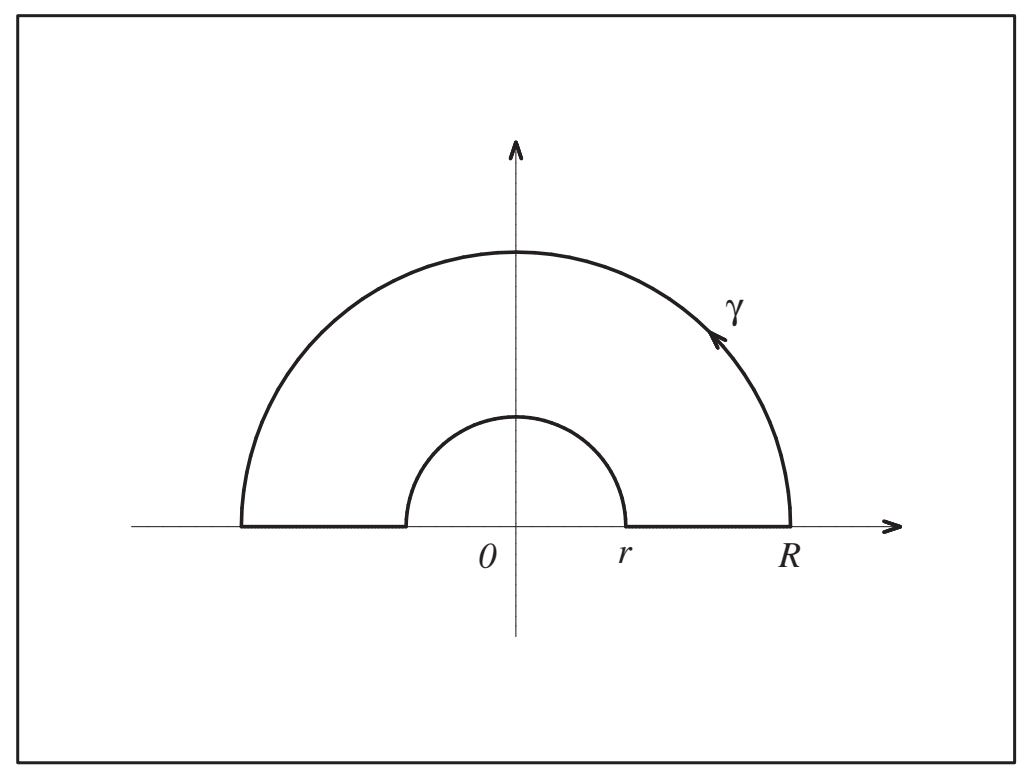

Figura 1.1:

Prova. (a) Note que se $\gamma$ é a curva da Figura 1.1 no plano complexo, pelo Teorema de Cauchy, temos que

$$
0=\int_{-R}^{-r} \frac{\mathbf{e}^{i t}}{t} d t+\int_{r}^{R} \frac{\mathbf{e}^{i t}}{t} d t+i \int_{\pi}^{0} \mathbf{e}^{i r \mathbf{e}^{i \theta}} d \theta+i \int_{0}^{\pi} \mathbf{e}^{i R \mathbf{e}^{i \theta}} d \theta
$$

Note que

$$
\left|\int_{0}^{\pi} \mathbf{e}^{i R \mathbf{e}^{i \theta}} d \theta\right| \leq \int_{0}^{\pi} \mathbf{e}^{-R \operatorname{sen} \theta} d \theta \rightarrow 0
$$

quando $R \rightarrow \infty$ e o resultado segue quando $r \rightarrow 0$.

(b)

$$
\int_{-1}^{1} \frac{\operatorname{sen} N t}{\pi t} d t=\int_{-N}^{N} \frac{\operatorname{sen} t}{\pi t} d t=\frac{1}{\pi} \int_{-N}^{N} \frac{\operatorname{sen} t}{t} d t \rightarrow 1, \quad \text { quando } \quad N \rightarrow \infty
$$

e

$$
\int_{-\infty}^{\infty} f(t) \frac{\operatorname{sen} N t}{\pi t} d t-f(0) \int_{-1}^{1} \frac{\operatorname{sen} N t}{\pi t} d t=\int_{|t| \leq 1} \frac{f(t)-f(0)}{\pi t} \operatorname{sen} N t d t+\int_{|t| \geq 1} \frac{f(t)}{\pi t} \operatorname{sen} N t d t
$$

Como $\int_{-1}^{1}\left|\frac{f(t)-f(0)}{t}\right| d t<\infty$, segue do Lema de Riemann-Lebesgue que

$$
\int_{-1}^{1} \frac{f(t)-f(0)}{\pi t} \operatorname{sen} N t d t \rightarrow 0, \quad \text { quando } \quad N \rightarrow \infty
$$


Agora, observe que como $\frac{f(t)}{\pi|t|}<\frac{f(t)}{1+|t|}$, para $|t| \geq 1$, e $\frac{f(t)}{1+|t|}$ é integrável em $\mathbb{R}$, por hipótese, temos que $\frac{f(t)}{\pi t} \in L^{1}((-\infty,-1] \cup[1, \infty))$. Segue então do Lema de Riemann-Lebesgue que

$$
\int_{|t| \geq 1} \frac{f(t)}{\pi t} \operatorname{sen} N t d t \rightarrow 0, \quad \text { quando } \quad N \rightarrow \infty
$$

Usando (1.24), (1.26) e (1.27) em (1.25) temos o resultado.

Teorema 1.16 Suponha que A é o gerador de um semigrupo fortemente contínuo $\{T(t): t \geq$ $0\} \subset L(E)$ satisfazendo $\|T(t)\|_{L(E)} \leq M \mathbf{e}^{\beta t}$ e admita que $\gamma>\max \{0, \beta\}$. Para qualquer $e \in D\left(A^{2}\right)$ e $t>0$

$$
T(t) e=\lim _{N \rightarrow \infty} \frac{1}{2 \pi i} \int_{\gamma-i N}^{\gamma+i N} \mathbf{e}^{\lambda t}(\lambda-A)^{-1} e d \lambda,
$$

onde a integral é ao longo do segmento de reta com $\operatorname{Re} \lambda=\gamma$. O limite converge uniformemente para $\epsilon \leq t \leq 1 / \epsilon$, qualquer $\epsilon>0$.

Prova. Como $\operatorname{Re} \lambda=\gamma>\beta,(\lambda-A)^{-1}$ existe e é uniformemente limitada. De fato, como $e \in D\left(A^{2}\right)$

$$
(\lambda-A)^{-1} e=\lambda^{-1} e+\lambda^{-2} A e+\lambda^{-2}(\lambda-A)^{-1} A^{2} e
$$

então

$$
\begin{aligned}
\frac{1}{2 \pi i} \int_{\gamma-i N}^{\gamma+i N} \mathbf{e}^{\lambda t}(\lambda-A)^{-1} e d \lambda & =\frac{1}{2 \pi i} \int_{\gamma-i N}^{\gamma+i N} \frac{\mathbf{e}^{\lambda t}}{\lambda} e d \lambda \\
& +\frac{1}{2 \pi i} \int_{\gamma-i N}^{\gamma+i N} \frac{\mathbf{e}^{\lambda t}}{\lambda^{2}}\left[A e+(\lambda-A)^{-1} A^{2} e\right] d \lambda
\end{aligned}
$$

e ambos os termos convergem uniformemente em $\epsilon \leq t \leq 1 / \epsilon$ quando $N \rightarrow \infty$, o primeiro por

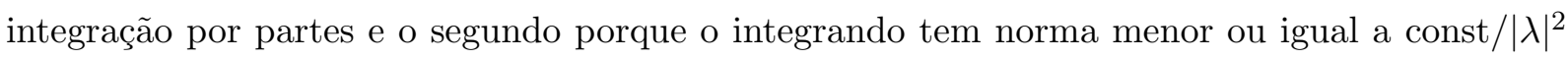
e então converge absolutamente. Resta mostrar que o limite é $T(t) e$.

Agora para $\operatorname{Re} \lambda=\gamma$

$$
(\lambda-A)^{-1} e=\int_{0}^{\infty} \mathbf{e}^{-\lambda s} T(s) e d s
$$

e então

$$
\begin{aligned}
\frac{1}{2 \pi i} \int_{\gamma-i N}^{\gamma+i N} \mathbf{e}^{\lambda t}(\lambda-A)^{-1} e d \lambda & =\int_{0}^{\infty}\left\{\frac{1}{2 \pi i} \int_{\gamma-i N}^{\gamma+i N} \mathbf{e}^{\lambda(t-s)} d \lambda\right\} T(s) e= \\
& =\int_{0}^{\infty} \frac{\operatorname{sen} N(t-s)}{\pi(t-s)} \mathbf{e}^{\gamma(t-s)} T(s) e d s= \\
& =\int_{-t}^{\infty} \frac{\operatorname{sen} N \tau}{\pi \tau} \mathbf{e}^{-\gamma \tau} T(t+\tau) e d \tau .
\end{aligned}
$$


A função

$$
f(\tau)= \begin{cases}\left\langle e^{*}, T(t+\tau) e\right\rangle \mathbf{e}^{-\gamma \tau}, & \tau \geq-t \\ 0, & \tau<-t\end{cases}
$$

satisfaz as condições do Lema 1.7 para qualquer $e^{*} \in E^{*}$ e $t>0$ pois $f^{\prime}(0)=\left\langle e^{*}, T(t)(A-\gamma) e\right\rangle$. Então

$$
\left\langle e^{*}, \frac{1}{2 \pi i} \int_{\gamma-i N}^{\gamma+i N} \mathbf{e}^{\lambda t}(\lambda-A)^{-1} e d \lambda\right\rangle \longrightarrow f(0)=\left\langle e^{*}, T(t) e\right\rangle
$$

quando $N \rightarrow \infty$. Isto vale para todo $e^{*} \in E^{*}$ e a prova está completa.

\subsection{Operadores setoriais e analiticidade}

Seja $A: D(A) \subset E \longrightarrow E$ o gerador de um semigrupo fortemente contínuo $\{T(t): t \geq 0\}$.

Definição 1.11 Se existem constantes a, $C$ e $\varphi \in(\pi / 2, \pi], \Sigma_{a, \varphi}=\{\lambda \in \mathbb{C}:|\arg (\lambda-a)|<\varphi\}$ está no conjunto resolvente de $A$ e

$$
\left\|(\lambda-A)^{-1}\right\|_{L(E)} \leq \frac{C}{|\lambda-a|} \quad \text { em } \Sigma_{a, \varphi}
$$

dizemos que $-A$ é setorial (com constante a).

Definição 1.12 Seja $\Delta=\left\{z: \varphi_{1}<\arg z<\varphi_{2}, \varphi_{1}<0<\varphi_{2}\right\} \subset \mathbb{C}$ e para $z \in \Delta$ seja $T(z)$ um operador linear limitado no espaço de Banach E. A família $\{T(z), z \in \Delta\}$ é um semigrupo analítico em $\Delta$ se:

(i) $z \mapsto T(z)$ é analítica em $\Delta$;

(ii) $T(0)=I_{E} e \lim _{z \in \Delta, z \rightarrow 0} T(z) e=e$, para todo $e \in E$;

(iii) $T\left(z_{1}+z_{2}\right)=T\left(z_{1}\right) T\left(z_{2}\right)$, para $z_{1}, z_{2} \in \Delta$.

Um semigrupo $\{T(t) ; t \geq 0\}$ será chamado de analítico se for analítico em algum setor $\Delta$ contendo o eixo real positivo.

Teorema 1.17 Se $A: D(A) \subset E \longrightarrow E$ é um operador linear, tal que $\Sigma=\{\lambda \in \mathbb{C}:|\arg \lambda|<$ $\varphi\} \subset \rho(A)$ para algum $\varphi \in(\pi / 2, \pi) e$

$$
\left\|(\lambda-A)^{-1}\right\|_{L(E)} \leq \frac{C}{|\lambda|}, \quad \lambda \in \Sigma,
$$

então o semigrupo gerado por A é analítico em um setor contendo o eixo real positivo. 
Prova. Seja $e \in D\left(A^{2}\right), t>0$, então para algum $\gamma>0$

$$
T(t) e=\frac{1}{2 \pi i} \int_{\gamma-i \infty}^{\gamma+i \infty} \mathbf{e}^{\lambda t}(\lambda-A)^{-1} e d \lambda .
$$

O integrando é analítico para $\lambda \in \Sigma$ e portanto podemos deformar o contorno de integração para $\Gamma$, consistindo de dois raios $\{\lambda \in \mathbb{C}: \arg \lambda= \pm \phi,|\lambda|>r\}, \frac{\pi}{2}<\phi<\varphi$, e do arco de círculo $\{\lambda \in \mathbb{C}:|\lambda|=r,|\arg \lambda| \leq \phi\}$ para $r$ pequeno. Veja Figura 1.2. Note que, quando $\operatorname{Im} \lambda= \pm N$, $-k N \leq \operatorname{Re} \lambda \leq \gamma(k=|\cot \phi|>0)$,

$$
\left\|\mathbf{e}^{\lambda t}(\lambda-A)^{-1} e\right\|_{E} \leq \frac{\mathbf{e}^{t \operatorname{Re} \lambda} C\|e\|_{E}}{\sqrt{(\operatorname{Re} \lambda)^{2}+N^{2}}}
$$

e as integrais correspondentes tendem a zero quando $N \rightarrow \infty$.

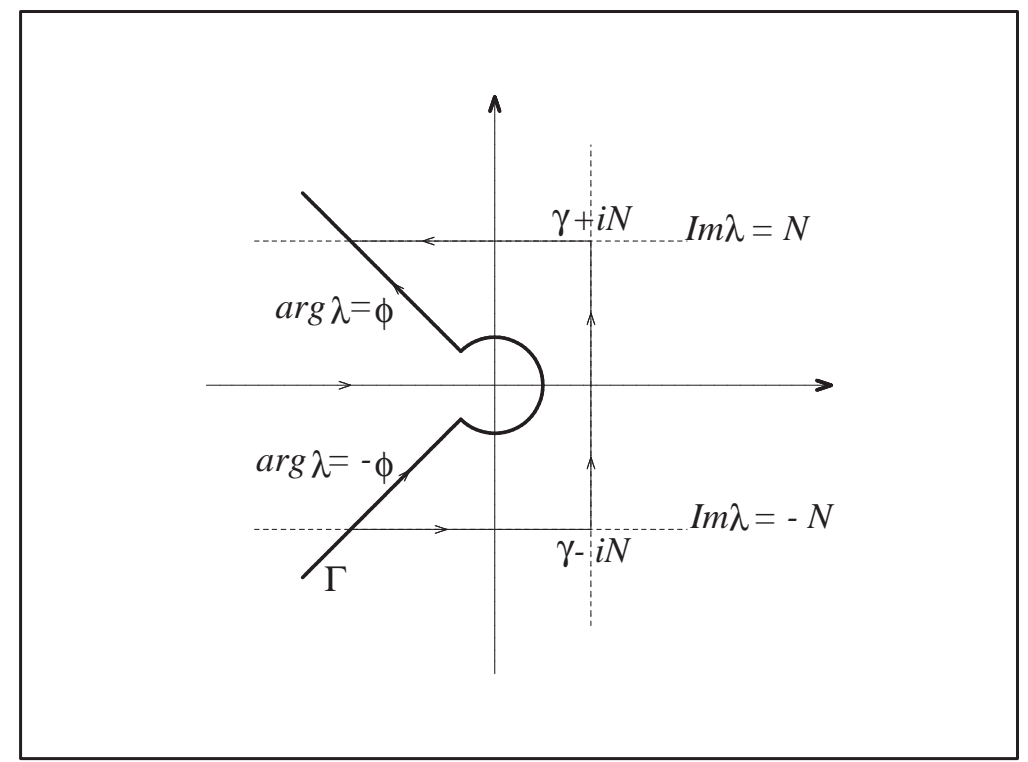

Figura 1.2:

Portanto,

$$
T(t) e=\frac{1}{2 \pi i} \int_{\Gamma} \mathbf{e}^{\lambda t}(\lambda-A)^{-1} e d \lambda,
$$

e esta expressão vale para todo $e \in E$ porque converge em norma. De fato, para $t>0, \arg \lambda= \pm \phi$

$$
\left\|\mathbf{e}^{\lambda t}(\lambda-A)^{-1}\right\|_{L(E)} \leq C \frac{\mathbf{e}^{-t|\lambda| k_{1}}}{|\lambda|}, \quad k_{1}=|\cos \phi|>0
$$

e então

$$
T(t)=\frac{1}{2 \pi i} \int_{\Gamma} \mathbf{e}^{\lambda t}(\lambda-A)^{-1} d \lambda
$$


com convergência na norma de $L(E)$ para qualquer $t>0$. Como vimos no Teorema 1.16, a convergência é uniforme para $\epsilon \leq t \leq \frac{1}{\epsilon}$, qualquer $\epsilon>0$ e então $t \mapsto T(t) \in L(E)$ é contínuo para $t>0$ (mas claramente a convergência não é uniforme quando $t \rightarrow 0$, a menos que $A$ seja limitado). Ainda mais, a integral converge uniformemente para $t$ complexo em $|\arg t| \leq \epsilon_{1}<\phi-\pi / 2$, $\epsilon_{0} \leq|t|,\left(\epsilon_{i}>0, i=0,1\right)$. Logo $t \mapsto T(t)$ é analítico em um setor $|\arg t|<\phi-\pi / 2$ contendo o eixo real positivo.

Esta prova de analiticidade não usa o fato que $A$ é o gerador de um semigrupo mas somente propriedades do resolvente $(\lambda-A)^{-1}$ quando $|\lambda| \rightarrow \infty$. De fato, qualquer operador densamente definido $A$ tal que $-A$ é setorial gera um semigrupo analítico. Isto é exatamente o que diz o próximo resultado.

Teorema 1.18 Suponha que $A: D(A) \subset E \rightarrow E$ é densamente definido e $-A$ é setorial. Então $A$ é o gerador infinitesimal de um semigrupo fortemente contínuo $\{T(t): t \geq 0\} \subset L(E)$,

$$
T(t)=\frac{1}{2 \pi i} \int_{\Gamma_{a}} \mathbf{e}^{\lambda t}(\lambda-A)^{-1} d \lambda
$$

onde $\Gamma_{a}$ é a fronteira de $\Sigma_{a, \phi} \backslash\{\lambda \in \mathbb{C}:|\lambda-a| \leq r\}, \frac{\pi}{2}<\phi<\varphi, r$ pequeno, orientada no sentido da parte imaginária crescente. Adicionalmente, $t \mapsto T(t)$ se estende a uma função analítica de $\{t \in \mathbb{C}:|\arg t|<\phi-\pi / 2\}$ em $L(E)$ (ou a complexificação de $E$, se $E$ é um espaço de Banach real) e para algum $K>0$

$$
\|T(t)\|_{L(E)} \leq K \mathbf{e}^{a t}, \quad\|A T(t)\|_{L(E)} \leq K t^{-1} \mathbf{e}^{a t}
$$

para todo $t>0$. Note que

$$
\frac{d}{d t} T(t)=A T(t)
$$

é um operador limitado para qualquer $t>0$.

Prova. Veja que não há perda de generalidade em assumir que $a=0$, pois se definirmos $T(t)$ por

$$
T(t)=\frac{1}{2 \pi i} \int_{\Gamma_{a}} \mathbf{e}^{\lambda t}(\lambda-A)^{-1} d \lambda
$$

tome $\lambda=a+\mu$, e então

$$
\mathbf{e}^{-a t} T(t)=\frac{1}{2 \pi i} \int_{\Gamma_{0}} \mathbf{e}^{\mu t}(\mu-(A-a))^{-1} d \mu
$$

$\operatorname{com} \|\left(\mu-(A-a)^{-1} \|_{L(E)} \leq C /|\mu|\right.$.

Como observado anteriormente, $t \mapsto T(t)$ se estende a uma função analítica de $\{t \in \mathbb{C}$ : $\left.|\arg t|<\phi-\frac{\pi}{2}\right\}$ em $L(E)$. Mostraremos primeiro que $\|T(t)\|_{L(E)}$ e $t\|A T(t)\|_{L(E)}$ são limitados 
para $t>0$. Mudando variáveis para $\mu=\lambda t$,

$$
T(t)=\frac{1}{2 \pi i} \int_{\Gamma_{0}} \mathbf{e}^{\mu}\left(\frac{\mu}{t}-A\right)^{-1} \frac{d \mu}{t},
$$

e o contorno é ainda $\Gamma_{0}$ já que o integrando é analítico. Logo

$$
\|T(t)\|_{L(E)} \leq \frac{1}{2 \pi} \int_{\Gamma_{0}} \mathbf{e}^{\operatorname{Re} \mu} \frac{C}{|\mu| / t} \frac{|d \mu|}{t}=K<\infty
$$

uniformemente para $t>0$. Semelhantemente

$$
\begin{aligned}
\frac{1}{2 \pi i} \int_{\Gamma_{0}} \mathbf{e}^{\lambda t} A(\lambda-A)^{-1} d \lambda & =\frac{1}{2 \pi i} \int_{\Gamma_{0}} \mathbf{e}^{\lambda t}\left[-I+\lambda(\lambda-A)^{-1}\right] d \lambda= \\
& =-\frac{1}{2 \pi i} \int_{\Gamma_{0}} \mathbf{e}^{\lambda t} d \lambda+\frac{t^{-1}}{2 \pi i} \int_{\Gamma_{0}} \mathbf{e}^{\mu} \frac{\mu}{t}\left(\frac{\mu}{t}-A\right)^{-1} d \mu
\end{aligned}
$$

onde o primeiro termo é zero e o segundo é estimado da seguinte forma

$$
\left\|\frac{t^{-1}}{2 \pi i} \int_{\Gamma_{0}} \mathbf{e}^{\mu} \frac{\mu}{t}\left(\frac{\mu}{t}-A\right)^{-1} d \mu\right\|_{L(E)} \leq \frac{1}{2 \pi} \frac{1}{t} \int_{\Gamma_{0}} \mathbf{e}^{\operatorname{Re} \mu} C|d \mu|=K_{1} t^{-1}<\infty .
$$

Para ver que isto é $A T(t)$, note que $A$ é um operador fechado, pois $(\lambda-A)^{-1} \in L(E)$ para $\lambda \in \Sigma_{0}$. Como a integral que define $T(t)$ é um limite de somas de Riemann é fácil ver que $A T(t) e=T(t) A e$ para todo $e \in D(A)$.

Pela analiticidade e convergência uniforme para cada $t>0$, temos

$$
\frac{d}{d t} T(t)=\frac{1}{2 \pi i} \int_{\Gamma_{0}} \mathbf{e}^{\lambda t} \lambda(\lambda-A)^{-1} d \lambda
$$

que é $A T(t)$ como mostrado acima. Sejam $e \in D(A), t>0$, então

$$
T(t) e=e+\frac{t}{2 \pi i} \int_{\Gamma_{0}} \mathbf{e}^{\mu} \frac{\mu}{t}\left(\frac{\mu}{t}-A\right)^{-1} A e \frac{d \mu}{\mu^{2}} .
$$

Logo,

$$
\|T(t) e-e\|_{E} \leq \frac{t}{2 \pi} \int_{\Gamma_{0}} \mathbf{e}^{\operatorname{Re} \mu} C\|A e\|_{E}\left|\frac{d \mu}{\mu^{2}}\right|=\mathcal{O}(t)
$$

quando $t \rightarrow 0^{+}$. Como $\|T(t)\|_{L(E)}$ é limitado quando $t \rightarrow 0^{+}, T(t) e \rightarrow e$ quando $t \rightarrow 0^{+}$para todo $e \in E$. Finalmente, para $0 \leq s \leq t$ a aplicação $s \mapsto T(t-s) T(s) e$ é contínua e é diferenciável (analítica) para $0<s<t$, com

$$
\frac{d}{d s}(T(t-s) T(s) e)=-A T(t-s) T(s) e+T(t-s) A T(s) e=0
$$

e então é constante satisfazendo

$$
T(t-s) T(s) e=T(t) e, \quad \text { para } \quad 0 \leq s \leq t, e \in E .
$$


Esta é a propriedade de semigrupo e a prova de que $T(t)$ é um semigrupo fortemente contínuo está completa. Para completar a prova do teorema, devemos mostrar que $A$ é seu gerador. Mas

$$
T(t) e-e=\int_{0}^{t} T(s) A e d s
$$

quando $t>0, e \in D(A)$, então $\frac{1}{t}(T(t) e-e) \rightarrow A e$ quando $t \rightarrow 0^{+}$e $A$ está contido no gerador. $A$ é de fato o gerador pois 1 está no resolvente de $A$ e do gerador.

Exemplo 1.1 Considere em $\mathbb{R}^{n}$ um domínio limitado $\Omega$ com fronteira $\partial \Omega$ suave e $1<p<\infty$. Considere também o operador $A_{0}: D\left(A_{0}\right) \subset L^{p}(\Omega) \rightarrow L^{p}(\Omega)$ definido por

$$
D\left(A_{0}\right)=\left\{u \in C^{2}(\bar{\Omega}): u=0 \text { sobre } \partial \Omega\right\} \text { e } A_{0} u=\Delta u=\sum_{k=1}^{n} \frac{\partial^{2} u}{\partial x_{k}^{2}}, \quad u \in D\left(A_{0}\right) .
$$

Então $A_{0}$ é fechável em $L^{p}(\Omega)$, e seu fecho A é o gerador de um semigrupo analítico de operadores lineares em $L^{p}(\Omega)$.

Prova. No que segue mostraremos que o operador $A_{0}$ é dissipativo, fechável, e seu fecho $A$ é também dissipativo.

Primeiramente observemos que se $0 \neq u \in D\left(A_{0}\right)$, ao considerarmos a função $u^{*}=\frac{|u|^{p-2} \bar{u}}{\|u\|_{L^{p}(\Omega)}^{p-2}}$, vemos que se $p^{*}=\frac{p}{p-1}$, então

$$
\begin{aligned}
\left\|u^{*}\right\|_{L^{p^{*}}(\Omega)} & =\left(\int_{\Omega}\left|u^{*}(x)\right|^{p^{*}} d x\right)^{\frac{1}{p^{*}}}=\frac{1}{\|u\|_{L^{p}(\Omega)}^{p-2}}\left(\int_{\Omega}\left(|u(x)|^{p-2}|\bar{u}(x)|\right)^{\frac{p}{p-1}} d x\right)^{\frac{p-1}{p}}= \\
& =\frac{1}{\|u\|_{L^{p}(\Omega)}^{p-2}}\left(\int_{\Omega}|u(x)|^{p} d x\right)^{\frac{p-1}{p}}=\|u\|_{L^{p}(\Omega)},
\end{aligned}
$$

e também

$$
\left\langle u^{*}, u\right\rangle=\frac{1}{\|u\|_{L^{p}(\Omega)}^{p-2}} \int_{\Omega} \bar{u}(x)|u(x)|^{p-2} u(x) d x=\frac{1}{\|u\|_{L^{p}(\Omega)}^{p-2}} \int_{\Omega}|u(x)|^{p} d x=\|u\|_{L^{p}(\Omega)}^{2} .
$$

Portanto $u^{*} \in J(u)$, onde $J: L^{p}(\Omega) \rightarrow 2^{L^{p^{*}}}$ denota a aplicação dualidade definida no Capítulo 1. Se $u=0$, colocamos $u^{*}=0$.

Em princípio consideramos o caso $2 \leq p<\infty$. Do Teorema da Divergência

$$
\begin{aligned}
-\int_{\Omega}\left(A_{0} u(x)\right)|u(x)|^{p-2} \bar{u}(x) d x & =\int_{\Omega}\left(\nabla u(x), \nabla\left(|u(x)|^{p-2} \bar{u}(x)\right)\right) d x= \\
& =\int_{\Omega}\left(|u(x)|^{p-2}(\nabla u(x), \nabla \bar{u}(x))\right. \\
& \left.+(p-2)|u(x)|^{p-3} \bar{u}(x)(\nabla u(x), \nabla|u(x)|)\right) d x
\end{aligned}
$$


onde $(\cdot, \cdot):\left(L^{p}(\Omega)\right)^{n} \times\left(L^{p}(\Omega)\right)^{n} \rightarrow \mathbb{R}$ é a forma bilinear anti-simétrica usual.

Uma vez que $|u|^{2}(\nabla u, \nabla \bar{u})=(\bar{u} \nabla u, u \nabla \bar{u})=|\operatorname{Re} \bar{u} \nabla u|^{2}+|\operatorname{Im} \bar{u} \nabla u|^{2}$, ao fazermos $u=\alpha+i \beta$, onde $\alpha, \beta$ são funções a valores reais de classe $C^{2}$, obtemos

$$
\begin{aligned}
\bar{u} \nabla u=(\alpha-i \beta)(\nabla \alpha+i \nabla \beta) & =(\alpha \nabla \alpha+\beta \nabla \beta)+i(\alpha \nabla \beta-\beta \nabla \alpha) \\
& =|u| \nabla|u|+i(\alpha \nabla \beta-\beta \nabla \alpha),
\end{aligned}
$$

onde usamos o fato de que como $|u|^{2}=\alpha^{2}+\beta^{2}$ então $|u| \nabla|u|=\alpha \nabla \alpha+\beta \nabla \beta$.

Portanto,

$$
\begin{aligned}
-\int_{\Omega}\left(A_{0} u\right)|u(x)|^{p-2}|\bar{u}(x)| d x & =\int_{\Omega}|u(x)|^{p-4}\left((p-1)|\operatorname{Re} \bar{u}(x) \nabla u(x)|^{2}+|\operatorname{Im} \bar{u}(x) \nabla u(x)|^{2}\right) d x \\
& +i \int_{\Omega}(p-2)|u(x)|^{p-4}(\operatorname{Re} \bar{u}(x) \nabla u(x), \operatorname{Im} \bar{u}(x) \nabla u(x)) d x,
\end{aligned}
$$

de onde concluímos que

$$
\operatorname{Re}\left(\left\langle v, A_{0} u\right\rangle\right) \leq 0, \quad \text { para algum } v \in J(u),
$$

qualquer que seja $u \in D\left(A_{0}\right)$.

No caso $1<p<2$, devemos ser mais cuidadosos ao aplicarmos o Teorema da Divergência, visto que $\bar{u}|u|^{p-2}$ deixa de ser de classe $C^{1}$ nos pontos onde $u$ se nula. Em princípio suponhamos $u$ de classe $C^{\infty}$. Neste caso a aplicação $x \mapsto|u(x)|^{2}$ é também de classe $C^{\infty}$ e portanto, pelo Teorema de Sard ${ }^{*}$, quase todo $\epsilon>0$ é valor regular de $|u(\cdot)|^{2}$, e dessa forma

$$
\Omega_{\epsilon}=\left\{x \in \Omega:|u(x)|^{2}>\epsilon\right\}
$$

possui fronteira suave. Podemos agora aplicar o Teorema da Divergência em $\Omega_{\epsilon}$, obtendo

$$
\int_{\Omega_{\epsilon}}\left(A_{0} u(x)\right) \bar{u}(x)|u(x)|^{p-2} d x=\int_{\partial \Omega_{\epsilon}} \bar{u}(x) \frac{\partial u(x)}{\partial \nu}|u(x)|^{p-2} d \sigma-\int_{\Omega_{\epsilon}}\left(\nabla u(x), \nabla\left(\bar{u}(x)|u(x)|^{p-2}\right) d x,\right.
$$

onde $\nu$ representa a normal unitária exterior a $\partial \Omega_{\epsilon}$.

Como visto anteriormente,

$$
\operatorname{Re}\left(\nabla u, \nabla\left(\bar{u}|u|^{p-2}\right)\right)=-|u|^{p-4}\left((p-1)|\operatorname{Re} \bar{u} \nabla u|^{2}+|\operatorname{Im} \bar{u} \nabla u|^{2}\right) \leq 0 .
$$

${ }^{*}$ Seja $f: \mathbb{R}^{n} \rightarrow \mathbb{R}^{p}$ uma aplicação suficientemente regular. Dizemos que $y \in \mathbb{R}^{p}$ é um valor regular para a aplicação $f$, se $f^{\prime}(x)$ for uma transformação linear sobrejetora sempre que $x \in f^{-1}(\{y\})$. Dessa forma, $y \in \mathbb{R}^{p}$ é um valor regular para $f$ se, ou $f^{-1}(\{y\})=\emptyset$ ou $f^{-1}(\{y\})$ é uma subvariedade suave de $\mathbb{R}^{n}$ de codimensão $p$. Dizemos que $y \in \mathbb{R}^{p}$ é um valor singular de $f$ se não for regular. Nestas condições temos o seguinte Teorema Teorema(Sard). Se $f: \mathbb{R}^{n} \rightarrow \mathbb{R}^{p}$ for uma aplicação suficientemente regular, então o conjunto dos valores singulares de $f$ tem medida nula em $\mathbb{R}^{p}$. 
Além disso, como $\nabla\left(|u|^{2}\right)=2|u| \nabla(|u|)$ é normal à superfície de nível $\epsilon,|u(x)|^{2}>\epsilon$ em $\Omega_{\epsilon} \mathrm{e}$ $|u(x)|^{2}=\epsilon$ em $\partial \Omega_{\epsilon}$, vemos que $\nu(x)=-\eta(x) \nabla(|u|(x))$, onde $\eta(x) \geq 0$ em $\partial \Omega_{\epsilon}$. E dessa forma,

$$
\operatorname{Re}\left(\bar{u} \frac{\partial u}{\partial \nu}\right)=\operatorname{Re}(\bar{u} \nabla u, \nu)=|u| \frac{\partial|u|}{\partial \nu} \leq 0 .
$$

Assim, para $u \in C^{\infty}(\Omega) \cap D\left(A_{0}\right)$,

$$
\operatorname{Re}\left(\int_{\Omega_{\epsilon}}\left(A_{0} u(x)\right) \bar{u}(x)|u(x)|^{p-2} d x\right) \leq 0
$$

para quase todo $\epsilon>0$. Fazendo $\epsilon \rightarrow 0^{+}$através dos valores regulares de $|u(\cdot)|^{2}$, obtemos

$$
\operatorname{Re}\left(\int_{\Omega}\left(A_{0} u(x)\right) \bar{u}(x)|u(x)|^{p-2} d x\right) \leq 0 .
$$

Agora, tomando-se limites na topologia $C^{2}$, obtemos que de fato $A_{0}$ é um operador dissipativo em $L^{p}(\Omega)$, para $1<p<\infty$.

Além disso, suponhamos que $\left\{u_{m}\right\}$ seja uma sequência em $D\left(A_{0}\right)$ tal que

$$
u_{m} \stackrel{L^{p}(\Omega)}{\longrightarrow} 0 \text { e } A_{0} u_{m} \stackrel{L^{p}(\Omega)}{\longrightarrow} v .
$$

Logo, para toda $\phi \in C_{o}^{\infty}(\Omega)$,

$$
\int_{\Omega} v(x) \phi(x) d x \stackrel{m \rightarrow \infty}{\longleftarrow} \int_{\Omega}\left(A_{0} u_{m}(x)\right) \phi(x) d x=-\int_{\Omega}\left(\nabla u_{m}(x), \nabla \phi(x)\right) d x \stackrel{m \rightarrow \infty}{\longrightarrow} 0,
$$

mostrando que $v=0$, ou seja, que $A_{0}$ é fechável em $L^{p}(\Omega)$.

Seja $A$ o fecho de $A_{0}$ em $L^{p}(\Omega)$. Segue do Teorema 1.8 que $A$ também é dissipativo. Assim, para aplicarmos o Teorema de Lumer-Phillips basta mostrar que $R(\lambda-A)=L^{p}(\Omega)$ para algum $\lambda>0$. Veja que, como $A$ é dissipativo, segue do Lema 1.2 que para todo $\lambda>0$ e $u \in D(A)$,

$$
\lambda\|u\|_{L^{p}(\Omega)} \leq\|(\lambda-A) u\|_{L^{p}(\Omega)} .
$$

Portanto vemos que $\lambda-A$ é um operador injetivo com imagem fechada para todo $\lambda>0$. De fato, suponhamos que $\left\{(\lambda-A) u_{n}\right\}$ seja uma sequência em $R(\lambda-A)$, a imagem do operador $\lambda-A$, tal que $(\lambda-A) u_{n} \rightarrow v$ em $L^{p}(\Omega)$. Pela desigualdade acima, a sequência $\left\{u_{n}\right\}$ é uma sequência de Cauchy em $L^{p}(\Omega)$ e dessa forma $u_{n} \stackrel{L^{p}(\Omega)}{\longrightarrow} u$, para alguma função $u \in L^{p}(\Omega)$. Sendo $\lambda-A$ fechado, $u \in D(\lambda-A)$ e $(\lambda-A) u=v$, concluímos que $R(\lambda-A)$ é de fato um subespaço fechado de $L^{p}(\Omega)$.

Basta mostramos então que para algum $\lambda>0, R(\lambda-A)$ é um subespaço denso de $L^{p}(\Omega)$ e então teremos mostrado que $R(\lambda-A)=L^{p}(\Omega)$. De fato, sendo $\partial \Omega$ uma hipersuperfície regular de $\mathbb{R}^{n}$, para cada $f \in C_{o}^{\infty}(\Omega)$, existe $u \in C^{2}(\bar{\Omega})$ tal que $(I-\Delta) u=f$ em $\Omega$, e $u \equiv 0$ em $\partial \Omega$. 
Agora veja que se $f \in L^{p}(\Omega)$, da densidade de $C_{o}^{\infty}(\Omega)$ em $L^{p}(\Omega)$, existe sequência $\left(f_{k}\right)$ em $C_{o}^{\infty}(\Omega)$ tal que $f_{k} \stackrel{k \rightarrow \infty}{\rightarrow} f$. Daí, como vimos, para cada $k$ existe $u_{k} \in D\left(A_{0}\right) \subset D(A)$ tal que

$$
(I-A) u_{k}=(I-\Delta) u_{k}=f_{k} \stackrel{k \rightarrow \infty}{\rightarrow} f
$$

mostrando que $f \in \overline{R(I-A)}$.

Portanto, pelo Teorema de Lumer-Phillips, o operador $A$ é o gerador de um semigrupo fortemente contínuo de contrações em $L^{p}(\Omega)$.

Por fim, se $J=\int_{\Omega}\left(A_{0} u(x)\right) \bar{u}(x)|u(x)|^{p-2} d x$, fazendo $v+i w=|u|^{\frac{u}{2}-2} \bar{u} \nabla u$, para cada $u \in D\left(A_{0}\right)$, vemos que

$$
-\operatorname{Re} J=\int_{\Omega}\left((p-1)|v(x)|^{2}+|w(x)|^{2}\right) d x \text { e }-\operatorname{Im} J=(p-2) \int_{\Omega}(v(x), w(x)) d x
$$

e consequentemente $\oplus^{\dagger}$

$$
\frac{|\operatorname{Im} J|}{-\operatorname{Re} J} \leq \frac{|p-2|}{2 \sqrt{p-1}} \frac{2\|\sqrt{p-1}|v|\|_{L^{2}(\Omega)}|||w| \|_{L^{2}(\Omega)}}{\int_{\Omega}\left((p-1)|v(x)|^{2}+|w(x)|^{2}\right) d x} \leq \frac{|p-2|}{2 \sqrt{p-1}} .
$$

Vemos portanto que a imagem numérica $W\left(A_{0}\right)$ está contida propriamente no setor do plano complexo $\Sigma_{p}^{\prime}=\left\{\lambda \in \mathbb{C}:|\operatorname{Im} \lambda|+\frac{|p-2|}{2 \sqrt{p-1}} \operatorname{Re} \lambda \leq 0\right\}$ se $1<p<\infty$ e $p \neq 2$, e em $\Sigma_{2}^{\prime}=(-\infty, 0]$ se $p=2$. Lembrando que se $A$ é o fecho de $A_{0}$, então $W(A)$ está contido no fecho de $W\left(A_{0}\right)$, e portanto também $W(A) \subset \Sigma_{p}^{\prime}$. Além disso, segue da Afirmação 3 do Apêndice $\mathbb{\text { A que }} \sigma(\Delta) \subset \mathbb{R}_{-}$. Assim, se considerarmos o conjunto

$$
\Sigma_{p}=\{\lambda \in \mathbb{C}:|\arg \lambda| \leq \varphi\},
$$

onde $\frac{\pi}{2}<\varphi<\frac{\pi}{2}+\delta \operatorname{com} \cot \delta=\frac{|p-2|}{2 \sqrt{p-1}}<\infty$, temos que $\Sigma_{p}$ é aberto e conexo em $\mathbb{C} \backslash W(A)$ e $\rho(A) \supset \Sigma_{p}$. Além disso, veja que para $\lambda \in \Sigma_{p}$,

$$
\operatorname{dist}(\lambda, W(A)) \geq \operatorname{dist}\left(\lambda, \Sigma_{p}\right) \geq \operatorname{Re} \lambda, \text { se } \operatorname{Re} \lambda>0,
$$

e

$$
\operatorname{dist}(\lambda, W(A)) \geq \operatorname{dist}\left(\lambda, \Sigma_{p}\right) \geq|\lambda| \cos (\varphi-\delta), \quad \text { se } \operatorname{Re} \lambda \leq 0 .
$$

Daí, segue do Teorema 1.7 que

$$
\left\|(\lambda-A)^{-1}\right\|_{L(E)} \leq \frac{1}{\operatorname{Re} \lambda} \text { se } \operatorname{Re} \lambda>0,
$$

e

$$
\left\|(\lambda-A)^{-1}\right\|_{L(E)} \leq \frac{1}{|\lambda| \cos (\varphi-\delta)}, \quad \text { se } \operatorname{Re} \lambda \leq 0 .
$$

Portanto $-A$ é um operador setorial, e o resultado segue do Teorema 1.17 .

\footnotetext{
${ }^{\dagger} 0 \leq\left\|v\left|\left\|_{L^{2}(\Omega)}^{2}+\right\|\right| w\left|\left\|_{L^{2}(\Omega)}^{2}-2\right\|\right| v\left|\left\|_{L^{2}(\Omega)}\right\| w\right|\right\|_{L^{2}(\Omega)}$
} 
Observação 1.1 Se $A: D(A) \subset L^{p}(\Omega) \rightarrow L^{p}(\Omega)$ é o operador (fecho de $A_{0}$ ) nas hipóteses do Exemplo 1.1 então, graças à densidade de $D\left(A_{0}\right)$ em $W^{2, p}(\Omega) \cap W_{0}^{1, p}(\Omega)$, temos $D(A)=$ $W^{2, p}(\Omega) \cap W_{0}^{1, p}(\Omega)$. 


\section{Capítulo 2}

\section{Potências fracionárias}

Neste capítulo apresentamos o conceito de potências fracionárias, bem como alguns resultados que caracterizam os espaços de potências fracionárias.

\subsection{Introdução}

Vamos começar esta seção motivando a definição de potências fracionárias de operadores fechados. Em primeiro lugar observe que se $\gamma$ é uma curva fechada, retificável e simples em $\mathbb{C} \backslash(-\infty, 0]$ e $n(\gamma ; a)$ denota o índice da curva $\gamma$ em $a \in \mathbb{C}$, temos do Teorema dos Resíduos que

$$
a^{\alpha}=\frac{1}{2 \pi i} \int_{\gamma} \frac{\lambda^{\alpha}}{\lambda-a} d \lambda
$$

para todo $\alpha \in \mathbb{R}$ e $a \in \mathbb{C} \operatorname{com} n(\gamma ; a)=1$. Aqui $\lambda^{\alpha}=\mathbf{e}^{\alpha \log \lambda}$ e $\log \lambda$ é o ramo principal do logarítimo.

Se $A \in L(E)$ é tal que $\sigma(A) \subset \mathbb{C} \backslash(-\infty, 0]$ e $\gamma$ é uma curva fechada, retificável e simples em $\mathbb{C} \backslash(-\infty, 0]$ tal que $n(\gamma ; a)=1, \forall a \in \sigma(A)$, definimos em analogia com a observação acima a potência fracionária do operador $A$ por

$$
A^{\alpha}=\frac{1}{2 \pi i} \int_{\gamma} \lambda^{\alpha}(\lambda-A)^{-1} d \lambda
$$

para todo $\alpha \in \mathbb{R}$. É fácil ver, da expressão acima, que $I^{\alpha}=I$ para todo $\alpha \in \mathbb{R}$.

É claro que $A^{\alpha} \in L(E)$. Mostremos que $A^{\alpha} A^{\beta}=A^{\alpha+\beta}\left(\left\{A^{\alpha}, \alpha \in \mathbb{R}\right\}\right.$ é um grupo) e que $A^{n}$ coincide com a definição usual (a $n$-ésima iterada de $A$ ). Para mostrar a propriedade de grupo 
escolha $\gamma^{\prime}$ uma curva fechada, retificável e simples em $\mathbb{C} \backslash(-\infty, 0]$ externa a $\gamma$, então

$$
\begin{aligned}
A^{\alpha} A^{\beta} & =\frac{1}{2 \pi i} \int_{\gamma} \lambda^{\alpha}(\lambda-A)^{-1} d \lambda \frac{1}{2 \pi i} \int_{\gamma^{\prime}} \mu^{\beta}(\mu-A)^{-1} d \mu= \\
& =\frac{1}{(2 \pi i)^{2}} \int_{\gamma^{\prime}} \int_{\gamma} \lambda^{\alpha} \mu^{\beta}(\lambda-A)^{-1}(\mu-A)^{-1} d \lambda d \mu= \\
& =\frac{1}{(2 \pi i)^{2}} \int_{\gamma^{\prime}} \int_{\gamma} \lambda^{\alpha} \mu^{\beta} \frac{(\lambda-A)^{-1}-(\mu-A)^{-1}}{\mu-\lambda} d \lambda d \mu= \\
& =\frac{1}{(2 \pi i)^{2}} \int_{\gamma} \lambda^{\alpha}(\lambda-A)^{-1} \int_{\gamma^{\prime}} \mu^{\beta} \frac{1}{\mu-\lambda} d \mu d \lambda+ \\
& +\frac{1}{(2 \pi i)^{2}} \int_{\gamma^{\prime}} \mu^{\beta}(\mu-A)^{-1} \int_{\gamma} \lambda^{\alpha} \frac{1}{\lambda-\mu} d \lambda d \mu= \\
& =\frac{1}{2 \pi i} \int_{\gamma} \lambda^{\alpha+\beta}(\lambda-A)^{-1} d \lambda=A^{\alpha+\beta}
\end{aligned}
$$

onde na última passagem utilizamos o Teorema dos Resíduos para obter que

$$
\frac{1}{2 \pi i} \int_{\gamma^{\prime}} \mu^{\beta} \frac{1}{\mu-\lambda} d \mu=\lambda^{\beta}
$$

e observamos que $\mu$ está no traço de $\gamma^{\prime}$ que é externa a $\gamma$ e portanto, do Teorema de Cauchy,

$$
\int_{\gamma} \lambda^{\alpha} \frac{1}{\lambda-\mu} d \lambda=0
$$

Se por outro lado $\alpha=n$ é um número inteiro positivo podemos tomar $\gamma$ uma curva em $\mathbb{C}$ (não é necessário evitar o semi-eixo real negativo), já que $\lambda \mapsto \lambda^{n}$ é uma função inteira, temos então que

$$
A_{n}:=\frac{1}{2 \pi i} \int_{\gamma} \lambda^{n}(\lambda-A)^{-1} d \lambda=\frac{1}{2 \pi i} \int_{\gamma} \lambda^{n-1}\left(I-\lambda^{-1} A\right)^{-1} d \lambda .
$$

Note que 0 é um polo de ordem $n$ do integrando e do Teorema dos Resíduos, obtemos que

$$
A_{n}=\frac{1}{2 \pi i} \int_{\gamma} \lambda^{n}(\lambda-A)^{-1} d \lambda=\operatorname{res}\left(\lambda^{n}(\lambda-A)^{-1}, 0\right) .
$$

Como para $|\lambda|>\|A\|_{L(E)}$ temos

$$
\left(I-\lambda^{-1} A\right)^{-1}=\sum_{j=0}^{\infty} \lambda^{-j} A^{j} .
$$

Obtemos assim a série de Laurent

$$
\lambda^{n-1}\left(I-\lambda^{-1} A\right)^{-1}=\sum_{j=0}^{\infty} \lambda^{(n-1)-j} A^{j},
$$

donde resulta que

$$
\operatorname{res}\left(\lambda^{n}(\lambda-A)^{-1}, 0\right)=A^{n}
$$


Concluímos então que

$$
A_{n}=A^{n},
$$

ou seja, $A^{\alpha}$ é a $\alpha$ iterada de $A$ quando $\alpha \in \mathbb{N}$.

No que segue buscaremos expressões equivalentes de $A^{\alpha}$ que façam sentido para uma classe mais ampla de operadores. Se $0<\phi<\pi$ e $A \in L(E)$ é tal que $\sigma(A) \subset \mathbb{C} \backslash(-\infty, 0]$, sejam $\Sigma_{\phi}=\{\lambda \in \mathbb{C}:|\arg \lambda|<\phi\}, B_{R}=\{\lambda \in \mathbb{C}:|\lambda|<R\}$ e $\Sigma_{R, \phi}=B_{R} \cap \Sigma_{\phi}$. Denote por $\Gamma_{R}$ a porção da fronteira de $\Sigma_{\phi}$ que está em $B_{R}$ orientada no sentido da parte imaginária decrescente, $\gamma_{R}$ a porção da fronteira de $B_{R}$ que está em $\Sigma_{\phi}$ orientada no sentido anti-horário. Com isto $\Gamma_{R}+\gamma_{R}$ é a fronteira de $\Sigma_{R, \phi}$. Escolha $R>2\|A\|$ e $\phi$ tal que $\Sigma_{R, \phi} \supset \sigma(A)$. Com isto temos que

$$
\begin{aligned}
A^{\alpha} & =\frac{1}{2 \pi i} \int_{\Gamma_{R}+\gamma_{R}} \lambda^{\alpha}(\lambda-A)^{-1} d \lambda= \\
& =\frac{1}{2 \pi i} \int_{\Gamma_{R}} \lambda^{\alpha}(\lambda-A)^{-1} d \lambda+\frac{1}{2 \pi i} \int_{\gamma_{R}} \lambda^{\alpha}(\lambda-A)^{-1} d \lambda
\end{aligned}
$$

e, sobre $\gamma_{R}$

$$
\left\|\lambda(\lambda-A)^{-1}\right\|_{L(E)}=\left\|\left(I-\lambda^{-1} A\right)^{-1}\right\|_{L(E)}=\left\|\sum_{n=0}^{\infty}\left(\frac{A}{\lambda}\right)^{n}\right\|_{L(E)} \leq \frac{1}{1-\frac{\|A\|_{L(E)}}{R}} \leq 2 .
$$

Se agora tomamos $\alpha<0$, vamos mostrar que a integral sobre $\gamma_{R}$ em (2.2) converge para zero quando $R$ tende para infinito. De fato,

$$
\left\|\int_{\gamma_{R}} \lambda^{\alpha}(\lambda-A)^{-1} d \lambda\right\|_{L(E)} \leq \int_{-\phi}^{\phi} R^{\alpha}\left\|\left(R e^{i \theta}-A\right)^{-1}\right\|_{L(E)} R d \theta
$$

e de (2.3) é fácil ver que a integral sobre $\gamma_{R}$ tende a zero quando $R$ tende para infinito.

Se $\Gamma$ denota a fronteira de $\Sigma_{\phi}$ orientada no sentido da parte imaginária decrescente, os cálculos acima mostram que sempre que $\alpha<0$, temos

$$
A^{\alpha}=\frac{1}{2 \pi i} \int_{\Gamma} \lambda^{\alpha}(\lambda-A)^{-1} d \lambda
$$

Observe que a convergência da integral em (2.4) somente depende da estimativa espectral em (2.3) e não do operador $A$. Isto segue facilmente se parametrizarmos $\Gamma$. Vamos apenas considerar a parte $\Gamma_{+}$de $\Gamma$ com parte imaginária positiva. Então

$$
\left\|\int_{\Gamma_{+}} \lambda^{\alpha}(\lambda-A)^{-1} d \lambda\right\|_{L(E)} \leq \int_{0}^{\infty} t^{\alpha}\left\|\left(t \mathbf{e}^{i \phi}-A\right)^{-1}\right\|_{L(E)} d t .
$$

Como o resolvente é contínuo sobre $\Gamma$ a convergência da integral acima segue somente de (2.3), ainda mais esta convergência é uniforme para $\alpha$ em qualquer compacto de $\{\lambda \in \mathbb{R}: \lambda<0\}$. A convergência da integral sobre a parte de $\Gamma$ com parte real negativa segue de forma semelhante. 
Esta observação nos indica uma classe mais geral de operadores $A$ para os quais podemos definir as potências $A^{\alpha}$ com $\alpha<0$. Esta classe é a classe dos operadores fechados, densamente definidos $A$ com resolvente contendo um setor $\mathbb{C} \backslash \Sigma_{\phi} \cup\{0\}=-\Sigma_{\pi-\phi} \cup\{0\}$ e tais que $\lambda(\lambda-A)^{-1}$ é limitada em $-\Sigma_{\pi-\phi-\epsilon}, \pi-\phi>\epsilon>0,0<\phi<\pi$.

Note que se $\varphi=\pi-\phi$ então $\lambda(\lambda-A)^{-1}$ é limitado em $-\Sigma_{\varphi}$ se, e somente se, $\lambda(\lambda+A)^{-1}$ é limitado em $\Sigma_{\varphi}$ se, e somente se, $(1+|\lambda|)\left\|(\lambda+A)^{-1}\right\|_{L(E)}$ é limitado em $\Sigma_{\varphi}$.

Mostramos a seguir que se $(1+s)\left\|(s+A)^{-1}\right\|_{L(E)} \leq M, s \in[0, \infty)$ então $(1+|\lambda|) \|(\lambda+$ $A)^{-1} \|_{L(E)}$ é limitado em $\Sigma_{\varphi}$ para $\varphi=\operatorname{arcsen} \frac{1}{2 M}$. Em particular, com isto teremos mostrado que podemos definir $A^{\alpha}$ através de (2.4) para todo operador $A$ tal que $-A$ gera um semigrupo fortemente contínuo $\{T(t): t \geq 0\}$ tal que $\|T(t)\|_{L(E)} \leq M, t \geq 0$.

\subsection{Operadores do tipo positivo}

Seja $E$ um espaço de Banach. Um operador linear $A$ em $E$ é dito de tipo positivo com constante $M$ (veja [1]), $M \geq 1$, se é fechado, densamente definido, $\mathbb{R}^{+} \subset \rho(-A) \mathrm{e}$

$$
(1+s)\left\|(s+A)^{-1}\right\|_{L(E)} \leq M, \quad s \in \mathbb{R}^{+} .
$$

Denotamos o conjunto dos operadores de tipo positivo por

$$
\mathcal{P}:=\mathcal{P}(E)
$$

Pelo restante desta seção admitimos que $A \in \mathcal{P}$.

Seja $A$ um operador de tipo positivo com constante $M$. Dados $s \in \mathbb{R}^{+}$e $\lambda \in \mathbb{C}$ satisfazendo

$$
|\lambda-s| \leq \frac{(1+s)}{2 M}
$$

segue de $\lambda+A=(s+A)\left(1+(\lambda-s)(s+A)^{-1}\right)$ que

$$
\lambda \in \rho(-A)
$$

$\mathrm{e}$

$$
\begin{aligned}
\left\|(\lambda+A)^{-1}\right\|_{L(E)} & \leq\left\|\left[1+(\lambda-s)(s+A)^{-1}\right]^{-1}\right\|_{L(E)}\left\|(s+A)^{-1}\right\|_{L(E)} \leq \\
& \leq\left\|1+(\lambda-s)(s+A)^{-1}\right\|^{-1} \frac{M}{1+s} \leq 2 M(1+s)^{-1} \leq \\
& \leq \frac{2 M}{1+|\lambda|} \frac{1+s+|\lambda-s|}{1+s} \leq \frac{2 M}{1+|\lambda|}\left(1+\frac{1}{2 M}\right)=\frac{2 M+1}{1+|\lambda|}
\end{aligned}
$$

Na Figura 2.1, $\theta \in\left(0, \operatorname{arcsen} \frac{1}{2 M}\right]$ e deduzimos que

$$
\Sigma_{M}:=\{z \in \mathbb{C}:|\arg z| \leq \operatorname{arcsen}[1 /(2 M)]\}+\{z \in \mathbb{C}:|z| \leq 1 /(2 M)\} \subset \rho(-A)
$$




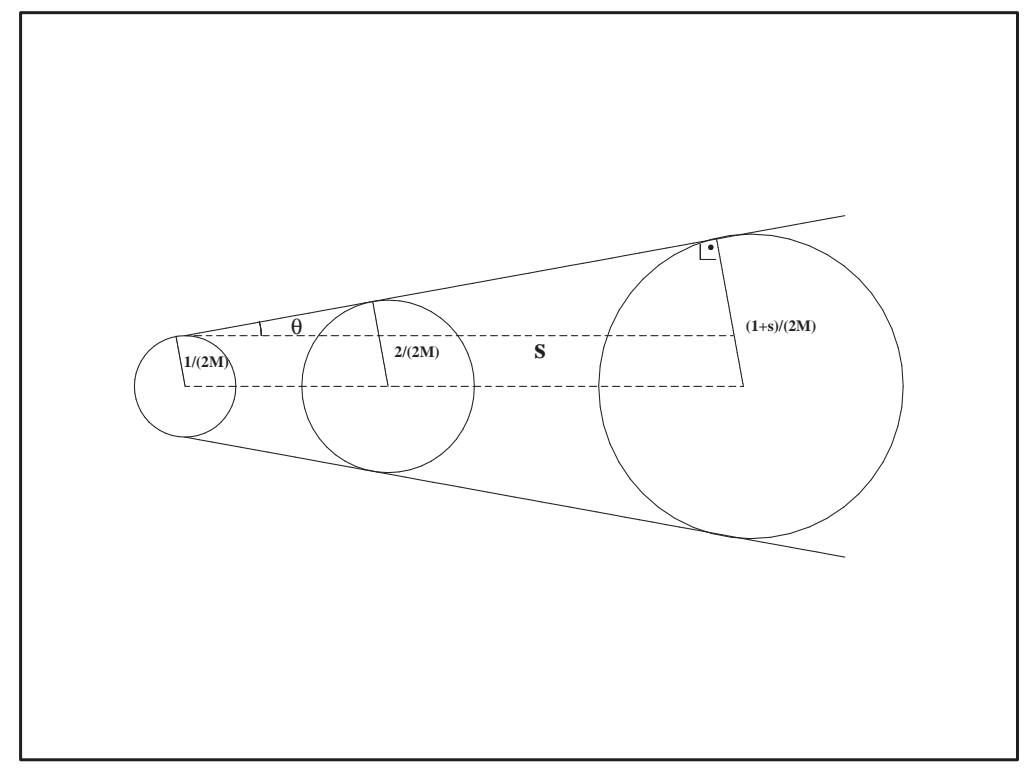

Figura 2.1:

e que

$$
(1+|\lambda|)\left\|(\lambda+A)^{-1}\right\|_{L(E)} \leq 2 M+1, \quad \lambda \in \Sigma_{M}
$$

Com isto para todo $A \in \mathcal{P}(E)$ e $\alpha \in \mathbb{R}$, com $\alpha<0$, definimos

$$
A^{\alpha}:=\frac{1}{2 \pi i} \int_{\Gamma}(-\lambda)^{\alpha}(\lambda+A)^{-1} d \lambda=-\frac{1}{2 \pi i} \int_{\tilde{\Gamma}} \lambda^{\alpha}(\lambda-A)^{-1} d \lambda
$$

onde $\Gamma$ é qualquer curva simples em $\Sigma_{M} \backslash \mathbb{R}^{+}$suave por partes indo de $\infty \mathbf{e}^{-i \nu}$ até $\infty \mathbf{e}^{i \nu}$ para algum $\nu \in(0, \operatorname{arcsen}(1 / 2 M)]$. É claro que $-\Gamma:=\{\lambda \in \mathbb{C}:-\lambda \in \Gamma\}$ e $\tilde{\Gamma}$ é uma curva com o mesmo traço de $-\Gamma$, mas percorrida em sentido contrário. Mostremos que a integral acima é convergente. De fato, para $r<\frac{1}{2 M}$ e $\nu \in\left(0, \operatorname{arcsen} \frac{1}{2 M}\right]$, temos

$$
\begin{aligned}
\frac{1}{2 \pi i} \int_{\Gamma}(-\lambda)^{\alpha}(\lambda+A)^{-1} d \lambda & =\frac{1}{2 \pi i} \int_{r}^{\infty}\left(-s \mathbf{e}^{i \nu}\right)^{\alpha}\left(s \mathbf{e}^{i \nu}+A\right)^{-1} \mathbf{e}^{i \nu} d s \\
& +\frac{1}{2 \pi i} \int_{\infty}^{r}\left(-s \mathbf{e}^{-i \nu}\right)^{\alpha}\left(s \mathbf{e}^{-i \nu}+A\right)^{-1} \mathbf{e}^{-i \nu} d s \\
& +\frac{1}{2 \pi i} \int_{2 \pi-\nu}^{\nu}\left(-r \mathbf{e}^{i \varphi}\right)^{\alpha}\left(r \mathbf{e}^{i \varphi}+A\right)^{-1} r i \mathbf{e}^{i \varphi} d \varphi
\end{aligned}
$$

e, observe ainda que se $\alpha \in \mathbb{R}$, então

$$
\left|\lambda^{\alpha}\right|=\left|\mathbf{e}^{\ln \lambda^{\alpha}}\right|=\left|\mathbf{e}^{\alpha(\ln |\lambda|+i \arg \lambda)}\right|=\mathbf{e}^{\alpha \ln |\lambda|}=|\lambda|^{\alpha} .
$$


Com isso, temos

$$
\begin{aligned}
\left\|\int_{r}^{\infty}\left(-s \mathbf{e}^{i \nu}\right)^{\alpha}\left(s \mathbf{e}^{i \nu}+A\right)^{-1} \mathbf{e}^{i \nu} d s\right\| & \leq \int_{r}^{\infty}\left|-s \mathbf{e}^{i \nu}\right|^{\alpha}\left\|\left(s \mathbf{e}^{i \nu}+A\right)^{-1}\right\| d s \leq \\
& \leq(2 M+1) \int_{r}^{\infty} s^{\alpha} \frac{1}{1+s} d s \leq \\
& \leq(2 M+1) \int_{r}^{\infty} s^{\alpha-1} d s<\infty .
\end{aligned}
$$

Ainda,

$$
\begin{aligned}
\left\|\int_{\infty}^{r}\left(-s \mathbf{e}^{-i \nu}\right)^{\alpha}\left(s \mathbf{e}^{-i \nu}+A\right)^{-1} \mathbf{e}^{-i \nu} d s\right\| & \leq \int_{r}^{\infty}\left|-s \mathbf{e}^{-i \nu}\right|^{\alpha}\left\|\left(s \mathbf{e}^{-i \nu}+A\right)^{-1}\right\| d s \leq \\
& \leq(2 M+1) \int_{r}^{\infty} s^{\alpha} \frac{1}{1+s} d s \leq \\
& \leq(2 M+1) \int_{r}^{\infty} s^{\alpha-1} d s<\infty,
\end{aligned}
$$

e

$$
\begin{aligned}
\left\|\int_{2 \pi-\nu}^{\nu}\left(-r \mathbf{e}^{i \varphi}\right)^{\alpha}\left(r \mathbf{e}^{i \varphi}+A\right)^{-1} r i \mathbf{e}^{i \varphi} d \varphi\right\| & \leq \int_{\nu}^{2 \pi-\nu} r^{\alpha}\left\|\left(r \mathbf{e}^{i \varphi}+A\right)^{-1}\right\| r d \varphi \leq \\
& \leq(2 M+1) \int_{\nu}^{2 \pi-\nu} \frac{r^{\alpha+1}}{1+r} d \varphi \leq \\
& \leq 2(2 M+1)(\pi-\nu) r^{\alpha+1}<\infty .
\end{aligned}
$$

Segue então que $A^{\alpha}$ está bem definido em $L(E)$ e independe da escolha de $\Gamma$. Ainda, mais é verdade.

Lema 2.1 Para todo $\alpha, \beta<0, A^{\alpha} A^{\beta}=A^{\alpha+\beta}$.

Prova. Dados $\alpha, \beta<0$, escolha $\Gamma_{1}$ e $\Gamma_{2}$ como em (2.7), de forma que $\Gamma_{1}$ fique a esquerda de $\Gamma_{2}$. Então

$$
\begin{aligned}
A^{\alpha} A^{\beta} & =\frac{1}{(2 \pi i)^{2}} \int_{\Gamma_{1}} \int_{\Gamma_{2}}(-\lambda)^{\alpha}(-\mu)^{\beta}(\lambda+A)^{-1}(\mu+A)^{-1} d \mu d \lambda= \\
& =\frac{1}{(2 \pi i)^{2}} \int_{\Gamma_{1}} \int_{\Gamma_{2}}(-\lambda)^{\alpha}(-\mu)^{\beta}(\lambda-\mu)^{-1}\left[(\mu+A)^{-1}-(\lambda+A)^{-1}\right] d \mu d \lambda= \\
& =\frac{1}{2 \pi i} \int_{\Gamma_{2}}(-\mu)^{\beta}(\mu+A)^{-1}\left(\frac{1}{2 \pi i} \int_{\Gamma_{1}} \frac{(-\lambda)^{\alpha}}{\lambda-\mu} d \lambda\right) d \mu+ \\
& +\frac{1}{2 \pi i} \int_{\Gamma_{1}}(-\lambda)^{\alpha}(\lambda+A)^{-1}\left(\frac{1}{2 \pi i} \int_{\Gamma_{2}} \frac{(-\mu)^{\beta}}{\mu-\lambda} d \mu\right) d \lambda .
\end{aligned}
$$

Para cada $\mu \in \Gamma_{2}$, a aplicação $\lambda \mapsto(\lambda-\mu)^{-1}(-\lambda)^{\alpha}$ é analítica sobre $\Gamma_{1}$ e à esquerda dela. Portanto, segue do Teorema de Cauchy que

$$
\frac{1}{2 \pi i} \int_{\Gamma_{1}} \frac{(-\lambda)^{\alpha}}{\lambda-\mu} d \lambda=0
$$


Além disso, segue de (2.7) e do Teorema dos Resíduos que

$$
\frac{1}{2 \pi i} \int_{\Gamma_{2}} \frac{(-\mu)^{\beta}}{\mu-\lambda} d \mu=(-\lambda)^{\beta} .
$$

Consequentemente,

$$
A^{\alpha} A^{\beta}=\frac{1}{2 \pi i} \int_{\Gamma_{1}}(-\lambda)^{\alpha+\beta}(\lambda+A)^{-1} d \lambda=A^{\alpha+\beta}
$$

o que prova a afirmativa.

Suponha que $0<\alpha<1$. Sabemos que

$$
A^{-\alpha}=-\frac{1}{2 \pi i} \int_{\tilde{\Gamma}} \lambda^{-\alpha}(\lambda-A)^{-1} d \lambda
$$

onde podemos escolher $\tilde{\Gamma} \subset \mathbb{C} / \mathbb{R}^{-}$, consistindo de:

$$
\left\{-s \mathbf{e}^{-i(\pi-\epsilon)}: s \in(-\infty,-r]\right\},\left\{r \mathbf{e}^{i \varphi}:|\varphi| \leq \pi-\epsilon\right\},\left\{s \mathbf{e}^{i(\pi-\epsilon)}: s \in[r, \infty)\right\}
$$

com $\epsilon>0$ e $r>0$ suficientemente pequenos. Segue então que

$$
\begin{aligned}
A^{-\alpha} & =-\frac{1}{2 \pi i} \int_{-\infty}^{-r}\left(-s \mathbf{e}^{-i(\pi-\epsilon)}\right)^{-\alpha}\left(-s \mathbf{e}^{-i(\pi-\epsilon)}-A\right)^{-1}\left(-\mathbf{e}^{-i(\pi-\epsilon)}\right) d s \\
& -\frac{1}{2 \pi i} \int_{-(\pi-\epsilon)}^{\pi-\epsilon}\left(r \mathbf{e}^{i \varphi}\right)^{-\alpha}\left(r \mathbf{e}^{i \varphi}-A\right)^{-1} r i \mathbf{e}^{i \varphi} d \varphi \\
& -\frac{1}{2 \pi i} \int_{r}^{\infty}\left(s \mathbf{e}^{i(\pi-\epsilon)}\right)^{-\alpha}\left(s \mathbf{e}^{i(\pi-\epsilon)}-A\right)^{-1} \mathbf{e}^{i(\pi-\epsilon)} d s .
\end{aligned}
$$

Observe que dado $r>0$, temos

$$
\mathbf{e}^{-\alpha(\ln |s|-i(\pi-\epsilon))}\left(-s \mathbf{e}^{-i(\pi-\epsilon)}-A\right)^{-1}\left(-\mathbf{e}^{-i(\pi-\epsilon)}\right) \stackrel{\epsilon \rightarrow 0}{\longrightarrow}(-s)^{-\alpha} \mathbf{e}^{i \pi \alpha}(s-A)^{-1},
$$

e

$$
\begin{aligned}
\left\|\mathbf{e}^{-\alpha(\ln |s|-i(\pi-\epsilon))}\left(-s \mathbf{e}^{-i(\pi-\epsilon)}-A\right)^{-1}\left(-\mathbf{e}^{-i(\pi-\epsilon)}\right)\right\| & \leq \mathbf{e}^{-\alpha \ln (-s)} \frac{2 M+1}{1-s} \leq \\
& \leq(2 M+1)(-s)^{-\alpha-1}
\end{aligned}
$$

onde a função dominante é integrável sobre $(-\infty,-r)$, pois $0<\alpha<1$. Então, usando o Teorema da Convergência Dominada, segue que

$$
\lim _{\epsilon \rightarrow 0} \int_{-\infty}^{-r} \mathbf{e}^{-\alpha(\ln |s|-i(\pi-\epsilon))}\left(-s \mathbf{e}^{-i(\pi-\epsilon)}-A\right)^{-1}\left(-\mathbf{e}^{-i(\pi-\epsilon)}\right) d s=\int_{-\infty}^{-r}(-s)^{-\alpha} \mathbf{e}^{i \pi \alpha}(s-A)^{-1} d s .
$$

Analogamente, para $r>0$,

$$
\mathbf{e}^{-\alpha(\ln s+i(\pi-\epsilon))}\left(s \mathbf{e}^{i(\pi-\epsilon)}-A\right)^{-1} \mathbf{e}^{i(\pi-\epsilon)} \stackrel{\epsilon \rightarrow 0}{\longrightarrow}-s^{-\alpha} \mathbf{e}^{-i \pi \alpha}(-s-A)^{-1}
$$


e

$$
\begin{aligned}
\left\|\mathbf{e}^{-\alpha(\ln s+i(\pi-\epsilon))}\left(s \mathbf{e}^{i(\pi-\epsilon)}-A\right)^{-1} \mathbf{e}^{i(\pi-\epsilon)}\right\| & \leq \mathbf{e}^{-\alpha \ln s} \frac{2 M+1}{1+s} \\
& \leq(2 M+1) s^{-\alpha-1},
\end{aligned}
$$

onde a função dominante é integrável sobre $(r, \infty)$, pois $0<\alpha<1$. Então, do Teorema da Convergência Dominada

$$
\int_{r}^{\infty} \mathbf{e}^{-\alpha(\ln s+i(\pi-\epsilon))}\left(s \mathbf{e}^{i(\pi-\epsilon)}-A\right)^{-1} \mathbf{e}^{i(\pi-\epsilon)} d s \stackrel{\epsilon \rightarrow 0}{\longrightarrow} \int_{r}^{\infty}-s^{-\alpha} \mathbf{e}^{-i \pi \alpha}(-s-A)^{-1} d s .
$$

Ainda, como

$$
\int_{-\pi}^{\pi}\left\|\mathbf{e}^{-\alpha(\ln r+i \varphi)}\left(r \mathbf{e}^{i \varphi}-A\right)^{-1} r i \mathbf{e}^{i \varphi}\right\| d \varphi \leq \frac{2 M+1}{1+r} r^{1-\alpha}<\infty
$$

obtemos que

$$
\lim _{\epsilon \rightarrow 0} \int_{-(\pi-\epsilon)}^{\pi-\epsilon} \mathbf{e}^{-\alpha(\ln r+i \varphi)}\left(r \mathbf{e}^{i \varphi}-A\right)^{-1} r i \mathbf{e}^{i \varphi} d \varphi=\int_{-\pi}^{\pi} \mathbf{e}^{-\alpha(\ln r+i \varphi)}\left(r \mathbf{e}^{i \varphi}-A\right)^{-1} r i \mathbf{e}^{i \varphi} d \varphi .
$$

Então, de (2.8), (2.9) e (2.10) temos que

$$
\begin{aligned}
A^{-\alpha} & =\frac{1}{2 \pi i} \int_{-\infty}^{-r}(-s)^{-\alpha} \mathbf{e}^{i \pi \alpha}(s-A)^{-1} d s- \\
& -\frac{1}{2 \pi i} \int_{-\pi}^{\pi} \mathbf{e}^{-\alpha(\ln r+i \varphi)}\left(r \mathbf{e}^{i \varphi}-A\right)^{-1} r i \mathbf{e}^{i \varphi} d \varphi- \\
& -\frac{1}{2 \pi i} \int_{r}^{\infty} s^{-\alpha} \mathbf{e}^{-i \pi \alpha}(s+A)^{-1} d s
\end{aligned}
$$

Além disso,

$$
\left\|\mathbf{e}^{-\alpha(\ln r+i \varphi)}\left(r \mathbf{e}^{i \varphi}-A\right)^{-1} r i \mathbf{e}^{i \varphi}\right\| \leq \frac{2 M+1}{1+r} r^{1-\alpha} \stackrel{r \rightarrow 0}{\longrightarrow} 0,
$$

pois, $1-\alpha>0$. Então, do Teorema da Convergência Dominada, resulta que

$$
\begin{aligned}
A^{-\alpha} & =\frac{1}{2 \pi i} \int_{0}^{\infty} s^{-\alpha} \mathbf{e}^{i \pi \alpha}(s+A)^{-1} d s-\frac{1}{2 \pi i} \int_{0}^{\infty} s^{-\alpha} \mathbf{e}^{-i \pi \alpha}(s+A)^{-1} d s= \\
& =\frac{1}{2 \pi i}\left(\mathbf{e}^{i \pi \alpha}-\mathbf{e}^{-i \pi \alpha}\right) \int_{0}^{\infty} s^{-\alpha}(s+A)^{-1} d s,
\end{aligned}
$$

isto é,

$$
A^{-\alpha}=\frac{\operatorname{sen} \pi \alpha}{\pi} \int_{0}^{\infty} s^{-\alpha}(s+A)^{-1} d s, \quad 0<\alpha<1
$$

Aplicando a fórmula (2.11) ao caso $E:=\mathbb{C}$ e $A:=1$, em particular, segue que

$$
\int_{0}^{\infty} s^{-\alpha}(1+s)^{-1} d s=\frac{\pi}{\operatorname{sen} \pi \alpha}, \quad 0<\alpha<1 .
$$

Portanto, deduzimos do fato que $A \in \mathcal{P}$ e da igualdade acima que

$$
\left\|A^{-\alpha}\right\|_{L(E)} \leq M \frac{|\operatorname{sen} \pi \alpha|}{\pi} \int_{0}^{\infty} s^{-\alpha}(1+s)^{-1} d s=M \frac{|\operatorname{sen} \pi \alpha|}{\operatorname{sen} \pi \alpha}=M
$$

para $0<\alpha<1$.

Agora não é difícil provar o seguinte resultado de continuidade: 
Teorema $2.1\left\{A^{\alpha}: \alpha<0\right\} \cup\left\{A^{0}=I_{E}\right\}$ é um semigrupo fortemente contínuo e analítico sobre E.

Prova. É uma consequência simples do teorema da derivação sob o sinal de integração que a aplicação $\alpha \mapsto A^{\alpha}$ é analítica em $\{\alpha \in \mathbb{R}: \alpha<0\}$. Portanto, graças ao Lema 2.1, resta mostrar que o semigrupo é fortemente contínuo em $\alpha=0$.

Note que

$$
(s+A)^{-1}-(1+s)^{-1}=(s+A)^{-1}\left(I-(1+s)^{-1}(s+A)\right)=(1+s)^{-1}(s+A)^{-1}(I-A),
$$

para $s>0$. Portanto, dado $e \in D(A)$ e $\alpha$ tal que $0<\alpha<1$, segue de (2.11) e de (2.12) que

$$
\begin{aligned}
A^{-\alpha} e-e & =\frac{\operatorname{sen} \pi \alpha}{\pi} \int_{0}^{\infty} s^{-\alpha}(s+A)^{-1} e d s-\frac{\operatorname{sen} \pi \alpha}{\pi} \int_{0}^{\infty} s^{-\alpha}(1+s)^{-1} e d s= \\
& =\frac{\operatorname{sen} \pi \alpha}{\pi} \int_{0}^{\infty} \frac{s^{-\alpha}}{1+s}(s+A)^{-1}(1-A) e d s .
\end{aligned}
$$

Consequentemente,

$$
\left\|A^{-\alpha} e-e\right\|_{E} \leq M \frac{|\operatorname{sen} \pi \alpha|}{\pi}\|(1-A) e\|_{E} \int_{0}^{\infty} \frac{s^{-\alpha}}{(1+s)^{2}} d s, \quad 0<\alpha<1 .
$$

Como a integral converge para 1 quando $\alpha \rightarrow 0$, vemos que $A^{-\alpha} e \rightarrow e$ quando $\alpha \rightarrow 0$. Assim como $A^{-\alpha}$ é uniformemente limitado para $0<\alpha<1$, graças a $(\overline{2.13}), A^{\alpha}$ converge para $I_{E}$ na topologia forte quando $\alpha \rightarrow 0^{-}$. Isto prova o teorema.

Lema 2.2 Seja $A: D(A) \subset E \longrightarrow E$ um operador positivo. Então, $A^{\alpha}$ é injetiva para $\alpha<0$.

Prova. Fixe $\alpha$ tal que $\alpha<0$. Suponha que $A^{\alpha} e=0$ para algum $e \in E$ e $\alpha<0$. Então segue do Teorema 2.1 que $A^{\alpha+\beta} e=A^{\beta} A^{\alpha} e=0$ para $\beta<0$. Portanto $A^{\beta} e=0$ para $\beta<\alpha$. Em particular, $A^{-k} e=0$ para $k=0,1,2, \cdots \operatorname{com} k>-\alpha$. Consequentemente, $e=0$. Isto mostra que $A^{\alpha}$ é injetiva para $\alpha<0$.

O Lema 2.2, nos diz que podemos definir as potências fracionárias de operadores positivos com expoente real positivo.

Definição 2.1 Seja $A: D(A) \subset E \longrightarrow E$ um operador positivo. Definimos a potência fracionária

$$
A^{\alpha}:=\left(A^{-\alpha}\right)^{-1},
$$

para $\alpha>0$. 
Denotaremos por $C(E):=C(E, E)$ o conjunto dos operadores lineares fechados de $E$ em $E$. Claramente, $A^{\alpha} \in C(E)$. Dados $\alpha, \beta \in \mathbb{R}$ com $0<\alpha<\beta$ e $e \in D\left(A^{\beta}\right)$, segue de

$$
e=A^{-\beta} A^{\beta} e=A^{-\alpha-(\beta-\alpha)} A^{\beta} e
$$

que $e \in D\left(A^{\alpha}\right)$, isto é,

$$
D\left(A^{\beta}\right) \subset D\left(A^{\alpha}\right), \quad 0<\alpha<\beta .
$$

Dado $e \in D(A)$, faça $f:=A e$. Como $D(A)$ é denso em $E$, podemos encontrar para cada $\epsilon>0$ um elemento $u \in D(A)$ tal que $\|u-f\|_{E} \leq \epsilon /\left\|A^{-1}\right\|_{L(E)}$. Portanto, fazendo $v:=A u$,

$$
\left\|A^{-2} v-e\right\|_{E}=\left\|A^{-1} u-A^{-1} f\right\|_{E} \leq\left\|A^{-1}\right\|_{L(E)}\|u-f\|_{E} \leq \epsilon .
$$

Isto mostra que $\overline{D\left(A^{2}\right)} \supset D(A)$. Portanto $\overline{D\left(A^{2}\right)} \supset \overline{D(A)}=E$ o que garante que $D\left(A^{2}\right)$ é denso em $E$. Por indução vemos que $D\left(A^{k}\right)$ é denso em $E$ para $k=1,2,3, \cdots$. Segue de (2.15) que

$$
\overline{D\left(A^{\alpha}\right)}=E, \quad \alpha>0 .
$$

Agora suponha que $\alpha>0$ e $\beta>0$. Dado

$$
e \in D\left(A^{\alpha+\beta}\right) \subset D\left(A^{\beta}\right) \cap D\left(A^{\alpha}\right),
$$

faça $f:=A^{\alpha+\beta} e$. Então

$$
e=A^{-(\alpha+\beta)} f=A^{-\beta} A^{-\alpha} f \Rightarrow A^{\beta} e=A^{-\alpha} f
$$

o que, por sua vez, mostra que $f=A^{\alpha} A^{\beta} e$; isto é,

$$
A^{\alpha+\beta} e=A^{\alpha} A^{\beta} e=A^{\beta} A^{\alpha} e, \quad e \in D\left(A^{\alpha+\beta}\right) .
$$

Se $\alpha>\beta$ e $e \in D\left(A^{\beta}\right)$ então

$$
A^{-\alpha} A^{\beta} e=A^{-(\alpha-\beta)} A^{-\beta} A^{\beta} e=A^{-(\alpha-\beta)} e=A^{\beta-\alpha} e .
$$

Adicionalmente, se $e \in D\left(A^{\alpha}\right)$ então, graças à identidade acima,

$$
A^{-\beta} A^{\alpha} e=A^{-\beta} A^{\beta} A^{\alpha-\beta} e=A^{\alpha-\beta} e .
$$

Isto prova que, dados $\alpha, \beta \in \mathbb{R} \operatorname{com} \alpha, \beta, \alpha+\beta \neq 0$,

$$
A^{\alpha} A^{\beta} e=A^{\alpha+\beta} e, \quad e \in D\left(A^{u}\right),
$$

onde $u=\max \{\alpha, \beta, \alpha+\beta\}$.

Considere a seguinte extensão de (2.11). 
Proposição 2.1 Suponha que $m=0,1,2, \cdots$. Então

$$
A^{-\alpha}=\frac{\operatorname{sen} \pi \alpha}{\pi} \frac{m !}{(1-\alpha)(2-\alpha) \cdots(m-\alpha)} \int_{0}^{\infty} s^{m-\alpha}(s+A)^{-m-1} d s
$$

para $0<\alpha<m+1$.

Prova. Suponha que $0<\alpha<1$. Então da integração por partes em (2.11) temos que,

$$
\begin{aligned}
A^{-\alpha} & =\frac{\operatorname{sen} \pi \alpha}{\pi(1-\alpha)}\left[\left.s^{1-\alpha}(s+A)^{-1}\right|_{0} ^{\infty}+\int_{0}^{\infty} s^{1-\alpha}(s+A)^{-2} d s\right]= \\
& =\frac{\operatorname{sen} \pi \alpha}{\pi(1-\alpha)} \int_{0}^{\infty} s^{1-\alpha}(s+A)^{-2} d s .
\end{aligned}
$$

Agora (2.18) segue por indução para $0<\alpha<1$. Graças a (2.5) é fácil verificar que a integral em (2.18) converge absolutamente para $0<\alpha<m+1$ e que o lado direito de (2.18) é uma aplicação analítica de $\{\alpha \in \mathbb{R}: 0<\alpha<m+1\}$ em $L(E)$. Agora a afirmativa segue do Teorema 2.1.

Agora suponha que $-1<\alpha<1$. Então pomos

$$
A_{\alpha} e:=\frac{\operatorname{sen} \pi \alpha}{\pi \alpha} \int_{0}^{\infty} s^{\alpha}(s+A)^{-2} A e d s, \quad e \in D(A)
$$

Observe que

$$
A_{0} e=\int_{0}^{\infty}(s+A)^{-2} d s A e=-\left.(s+A)^{-1} A e\right|_{0} ^{\infty}=e, \quad e \in D(A) .
$$

Adicionalmente, se $\alpha \neq 0$, segue de $(2.11)$ e de (2.18) que

$$
\begin{aligned}
A^{\alpha} e & =A^{\alpha-1} A e=\frac{\operatorname{sen} \pi(1-\alpha)}{\pi \alpha} \int_{0}^{\infty} s^{\alpha}(s+A)^{-2} A e d s= \\
& =\frac{\operatorname{sen} \pi \alpha}{\pi \alpha} \int_{0}^{\infty} s^{\alpha}(s+A)^{-2} A e d s=A_{\alpha} e,
\end{aligned}
$$

para $e \in D(A)$. Note que

$$
A^{-1} A_{\alpha} \subset B_{\alpha}:=\frac{\operatorname{sen} \pi \alpha}{\pi \alpha} \int_{0}^{\infty} s^{\alpha}(s+A)^{-2} d s \in L(E) .
$$

Agora, seja $\left(e_{j}\right)$ uma seqüência em $D(A)$ tal que $e_{j} \rightarrow 0$ e $A_{\alpha} e_{j} \rightarrow f$ em $E$. Então graças a (2.21), $B_{\alpha} e_{j} \rightarrow 0$ e $B_{\alpha} e_{j} \rightarrow A^{-1} f$, o que implica que $f=0$. Portanto $A_{\alpha}$ é fechável. Motivados por $(\overline{2.19})$ e (2.20) fazemos

$$
A^{\alpha}:=\text { fecho de } A_{\alpha}, \quad \alpha \neq 0
$$

Daqui por diante, frequentemente escreveremos $D\left(A^{\alpha}\right)$ para denotar este espaço vetorial munido com a norma do gráfico de $A^{\alpha}$. Escreveremos $I s(X, Y)$ para denotar o subespaço de $L(X, Y)$ consistindo dos isomorfismos lineares de $X$ sobre $Y$. Com estas considerações já provamos a maior parte do seguinte teorema. 
Teorema 2.2 Suponha que $A \in \mathcal{P}(E)$. Então a potência fracionária $A^{\alpha}$ é, para cada $\alpha \in \mathbb{R}$, um operador linear fechado densamente definido em $E$. Se $\alpha<0$ então $A^{\alpha} \in L(E)$ e é dado pela integral

$$
A^{\alpha}=\frac{1}{2 \pi i} \int_{\Gamma}(-\lambda)^{\alpha}(\lambda+A)^{-1} d \lambda
$$

onde $\Gamma$ é qualquer curva simples suave por partes em $\mathbb{C} \backslash \mathbb{R}^{+}$indo de $\infty \mathbf{e}^{-i \varphi}$ a $\infty \mathbf{e}^{i \varphi}$ para algum $\varphi \in(0, \pi)$ tal que $\sigma(-A)$ fica estritamente a esquerda de $\Gamma$. Adicionalmente,

(i) $A^{\alpha}$ é a potência usual de $A$ se $\alpha$ é inteiro.

(ii) $A^{\alpha} e=\frac{\operatorname{sen} \pi \alpha}{\pi \alpha} \int_{0}^{\infty} s^{\alpha}(s+A)^{-2} A e d s, \quad e \in D(A), \quad-1<\alpha<1$.

(iii) Suponha que ou $m=0,1,2, \cdots, e \in D\left(A^{2 m}\right)$ e $\max \{\alpha, \beta\}<m$ ou $\alpha, \beta$ e $\alpha+\beta$ não são nulos e e $\in D\left(A^{u}\right)$ onde $u=\max \{\alpha, \beta, \alpha+\beta\}$. Então

$$
A^{\alpha} A^{\beta} e=A^{\alpha+\beta} e .
$$

(iv) $A^{\alpha} A^{\beta}=A^{\alpha+\beta}, \alpha, \beta>0$.

(v) $D\left(A^{\beta}\right) \stackrel{d}{\hookrightarrow} D\left(A^{\alpha}\right) \stackrel{d}{\hookrightarrow} E, 0<\alpha<\beta$.

(vi) $A^{\alpha} \in I s\left(D\left(A^{\alpha+\beta}\right), D\left(A^{\beta}\right)\right) \cap I s\left(D\left(A^{\alpha}\right), E\right), \alpha, \beta>0$.

(vii) Dado $m=0,1,2, \cdots$, a aplicação

$$
\{\alpha \in \mathbb{R}: \alpha<m\} \rightarrow L\left(D\left(A^{m}\right), E\right), \quad \alpha \mapsto A^{\alpha}
$$

é analítica.

Prova. A primeira parte da afirmativa segue de resultados que precedem o enunciado do teorema.

(i) Segue de (2.1) e de (2.14).

(ii) Se $\alpha \neq 0$, isto foi mostrado em $(2.20)$ e segue da definição de $A^{\alpha}$ se $\alpha=0$.

(iii) Se $\alpha, \beta$ e $\alpha+\beta$ são todos distintos de zero, isto é uma consequência de (2.17) e (2.15). De (ii) e (2.5) concluímos que

$$
\left(\alpha \mapsto A^{\alpha}\right) \in C^{1}\left(\{\alpha \in \mathbb{R}:-1<\alpha<1\}, L(D(A), E) \cap L\left(D\left(A^{2}\right), D(A)\right)\right) .
$$

Portanto, suponha que $\alpha, \beta \in\{\zeta \in \mathbb{R}:-1<\zeta<1\}$. Escolha as sequências $\left(\alpha_{j}\right),\left(\beta_{j}\right)$ em

$$
\{\alpha \in \mathbb{R}:-1<\alpha<1\} \backslash\{\alpha \in \mathbb{R}: \alpha=0\}=: \mathcal{A}
$$


tal que $\alpha_{j}+\beta_{j} \in \mathcal{A}, \alpha_{j} \rightarrow \alpha$ e $\beta_{j} \rightarrow \beta$. Então, pelo que já sabemos,

$$
A^{\alpha_{j}} A^{\beta_{j}} e=A^{\alpha_{j}+\beta_{j}} e, \quad e \in D\left(A^{2}\right) .
$$

Portanto, fazendo $j \rightarrow \infty$, obtemos de (2.23) que (iii) é verdade se $-1<\alpha, \beta<1$.

Suponha que $\alpha=0$ e $\{\beta \in \mathbb{R}:|\beta| \geq 1\}$. Fixe $\rho \in \mathbb{R}$ com $0<\rho-\beta<1$. Então

$$
A^{\alpha} A^{\beta} e=A^{\alpha} A^{\beta-\rho} A^{\rho} e=A^{\alpha+(\beta-\rho)} A^{\rho} e=A^{(\alpha+\beta-\rho)+\rho} e=A^{\alpha+\beta} e,
$$

para $e \in D\left(A^{2 m}\right)$ com $m=2,3, \cdots$ e $\beta<m$ já que $-1<\beta-\rho<0$ e $\rho \neq 0$.

Finalmente, seja $\alpha \leq-1,1 \leq \beta$ e $\alpha+\beta=0$. Escrevemos $\alpha=r+s$ com $-1<r<0$. Como $s, \beta$ e $s+\beta$ são não nulos e $\alpha, r$ e $s$ são negativos, segue que

$$
A^{\alpha} e=A^{r} A^{s} e \text { e } A^{s} A^{\beta} e=A^{s+\beta} e \text { para } e \in D\left(A^{\beta}\right) .
$$

Portanto

$$
A^{\alpha} A^{\beta} e=A^{r} A^{s+\beta} e, \quad e \in D\left(A^{\beta}\right) \subset D\left(A^{2 m}\right) .
$$

Logo podemos assumir que $-1<\alpha<0$. Então $\alpha+\beta=0$ implica $0<\beta<1$, de forma que estamos de volta a situação já considerada. Consequentemente, (iii) foi completamente provado.

(iv) Pelo Teorema 2.1 e (iii) é suficiente provar que $e \in D\left(A^{\beta}\right)$ e $A^{\beta} e \in D\left(A^{\alpha}\right)$ implicam $e \in D\left(A^{\beta+\alpha}\right)$ se $\alpha, \beta>0$. Seja $f:=A^{\alpha}\left(A^{\beta} e\right)$. Então segue de (iii) que

$$
e=A^{-\beta}\left(A^{-\alpha} f\right)=A^{-(\beta+\alpha)} f \in D\left(A^{\beta+\alpha}\right) .
$$

(v) De (2.15) e de (iii) deduzimos que se $0<\alpha<\beta$ então

$$
\left\|A^{\alpha} e\right\|_{E}=\left\|A^{\alpha-\beta} A^{\beta} e\right\|_{E} \leq\left\|A^{\alpha-\beta}\right\|_{L(E)}\left\|A^{\beta} e\right\|_{E}, \quad e \in D\left(A^{\beta}\right) .
$$

Como $e \mapsto\left\|A^{u} e\right\|_{E}$ é uma norma equivalente a norma em $D\left(A^{u}\right)$ para $u>0$, graças a limitação de $A^{-u}$, segue que $D\left(A^{\beta}\right) \hookrightarrow D\left(A^{\alpha}\right) \hookrightarrow E$.

Dado $e \in D\left(A^{\alpha}\right)$ faça $f:=A^{\alpha} e \in E$. Como $D\left(A^{\beta-\alpha}\right)$ é denso em $E$, dado $\epsilon>0$ podemos encontrar $u \in D\left(A^{\beta-\alpha}\right)$ tal que $\|u-f\|_{E}<\epsilon$. Portanto

$$
v:=A^{-\alpha} u \in D\left(A^{\beta}\right) \quad \text { e } \quad\left\|A^{\alpha}(v-e)\right\|_{E}=\|u-f\|_{E}<\epsilon .
$$

Isto mostra que $D\left(A^{\beta}\right)$ é denso em $D\left(A^{\alpha}\right)$ que, junto com (2.16), implica a afirmativa.

(vi) A primeira afirmativa segue de (iv) e a segunda é trivial. 
(vii) Graças ao Teorema 2.1 e (2.23), podemos assumir que $m \geq 2$. Desde que (v) implica

$$
L(D(A), E) \hookrightarrow L\left(D\left(A^{m}\right), E\right),
$$

concluímos que

$$
\left(\alpha \mapsto A^{\alpha}\right) \in C^{1}\left(\{\alpha \in \mathbb{R} ; \alpha<1\}, L\left(D\left(A^{m}\right), E\right)\right) .
$$

Se $0<\alpha<m$ então (iii) implica que $A^{\alpha} e=A^{\alpha-m} A^{m} e$ para $e \in D\left(A^{m}\right)$. Portanto o Teorema 2.1 garante que

$$
\left(\alpha \mapsto A^{\alpha}\right) \in C^{1}\left(\{\alpha \in \mathbb{R} ; 0<\alpha<m\}, L\left(D\left(A^{m}\right), E\right)\right) .
$$

Isto juntamente com (2.25) prova o teorema.

Dados $M \geq 1$ e $\sigma \in \mathbb{R}$, denotemos por $\mathcal{G}(E, M, \sigma)$ o conjunto de todas $A \in C(E)$ tal que $-A$ é o gerador de um semigrupo fortemente contínuo $\left\{\mathbf{e}^{-t A}: t \geq 0\right\}$ em $E$ satisfazendo

$$
\left\|\mathbf{e}^{-t A}\right\|_{L(E)} \leq M \mathbf{e}^{\sigma t}, t \geq 0
$$

Note que $\mathcal{G}(E, M, \sigma) \subset P(E)$ para $\sigma>0$, isto é, se $-A$ é o gerador infinitesimal de um semigrupo fortemente contínuo com decaimento exponencial em $E$ então $A$ é do tipo positivo. Neste caso podemos obter outra fórmula de representação útil para $A^{\alpha} \operatorname{com} \alpha>0$.

Teorema 2.3 Suponha que $A \in \mathcal{G}(E, M, \sigma)$, para algum $M \geq 1$ e $\sigma>0$. Então

$$
A^{-\alpha}=\frac{1}{\Gamma(\alpha)} \int_{0}^{\infty} t^{\alpha-1} \mathbf{e}^{-t A} d t, \quad \alpha>0 .
$$

Prova. É uma consequência fácil de

$$
\left\|\int_{0}^{\infty} t^{\alpha-1} \mathbf{e}^{-t A} d t\right\|_{L(E)} \leq M \int_{0}^{\infty} t^{\alpha-1} \mathbf{e}^{-\sigma t} d t
$$

e das propriedades conhecidas da função $\Gamma$ que a aplicação

$$
\{\alpha \in \mathbb{R}: \alpha>0\} \rightarrow L(E), \quad \alpha \mapsto \frac{1}{\Gamma(\alpha)} \int_{0}^{\infty} t^{\alpha-1} \mathbf{e}^{-t A} d t
$$

é analítica. Portanto, graças ao Teorema 2.1 e ao Teorema da identidade de funções analíticas, é suficiente provar a igualdade para $0<\alpha<1$. Dado $\alpha \in(0,1)$,

$$
A^{-\alpha}=\frac{\operatorname{sen} \pi \alpha}{\pi} \int_{0}^{\infty} s^{-\alpha}(s+A)^{-1} d s
$$

pela Proposição 2.1. Por outro lado, segue do Teorema 1.2 que

$$
(s+A)^{-1}=\int_{0}^{\infty} \mathbf{e}^{-s t} \mathbf{e}^{-t A} d t, \quad s>0 .
$$


Portanto pelo Teorema de Fubini

$$
\begin{aligned}
A^{-\alpha} & =\frac{\operatorname{sen} \pi \alpha}{\pi} \int_{0}^{\infty} s^{-\alpha} \int_{0}^{\infty} \mathbf{e}^{-s t} \mathbf{e}^{-t A} d t d s=\frac{\operatorname{sen} \pi \alpha}{\pi} \int_{0}^{\infty} \mathbf{e}^{-t A} \int_{0}^{\infty} s^{-\alpha} \mathbf{e}^{-t s} d s d t= \\
& =\frac{\operatorname{sen} \pi \alpha}{\pi} \Gamma(1-\alpha) \int_{0}^{\infty} t^{\alpha-1} \mathbf{e}^{-t A} d t
\end{aligned}
$$

Assim a afirmativa segue da fórmula

$$
\Gamma(\alpha) \Gamma(1-\alpha)=\frac{\pi}{\operatorname{sen} \pi \alpha}
$$

que é consequência da relação anterior para $A=I$.

\subsection{Desigualdades de interpolação para potências fracionárias}

Nesta seção mostramos que $\left\|A^{\alpha} e\right\|_{E} \leq K\|A e\|_{E}^{\alpha}\|e\|_{\mathrm{E}}^{1-\alpha}$ para todo $0 \leq \alpha \leq 1, e \in D(A)$ e lidamos com perturbações $B$ de operadores positivos $A$ subordinados às potências fracionárias $A^{\alpha}$ de $A$.

Teorema 2.4 Suponha que $A \in \mathcal{P}(E)$ e $0 \leq \alpha \leq 1$, então

$$
\left\|(\mu+A)^{-1} e\right\|_{E} \leq K \mu^{\alpha-1}\left\|A^{-\alpha} e\right\|_{E}, \quad \mu>0, e \in E .
$$

Aqui $K$ é uma constante dependendo de $M$.

Prova. Sabemos que $\left\|s(s+A)^{-1}\right\|_{L(E)} \leq M,\left\|A(s+A)^{-1}\right\|_{L(E)} \leq M+1, s \geq 0$. Logo, para $\mu>0$ e $e \in E$

$$
\left\|(\mu+A)^{-1} e\right\|_{E} \leq\left\|(\mu+A)^{-1}\right\|_{L(E)}\|e\|_{E} \leq M \mu^{-1}\|e\|_{E}
$$

e (2.26) segue para $\alpha=0$ e $K=M$. Por outro lado,

$$
\left\|(\mu+A)^{-1} e\right\|_{E}=\left\|A(\mu+A)^{-1} A^{-1} e\right\|_{E} \leq\left\|A(\mu+A)^{-1}\right\|_{L(E)}\left\|A^{-1} e\right\|_{E} \leq(M+1)\left\|A^{-1} e\right\|_{E}
$$

e (2.26) segue para $\alpha=1$ e $K=M+1$.

Agora, suponha $0<\alpha<1$. Seja $e \in D(A)$, então segue de (2.11) que

$$
\begin{aligned}
(\mu+A)^{-1} e & =A^{\alpha-1} A(\mu+A)^{-1} A^{-\alpha} e= \\
& =\frac{\operatorname{sen} \pi \alpha}{\pi} \int_{0}^{\infty} s^{\alpha-1} A(\mu+A)^{-1}(s+A)^{-1} A^{-\alpha} e d s
\end{aligned}
$$


Portanto,

$$
\begin{aligned}
\left\|(\mu+A)^{-1} e\right\|_{E} & \leq \frac{\operatorname{sen} \pi \alpha}{\pi} M(M+1)\left[\int_{0}^{\mu} s^{\alpha-1} d s \mu^{-1}+\int_{\mu}^{\infty} s^{\alpha-2} d s\right]\left\|A^{-\alpha} e\right\|_{E} \leq \\
& \leq M(M+1) \frac{\operatorname{sen} \pi \alpha}{\pi}\left[\frac{1}{\alpha} \mu^{\alpha-1}+\frac{1}{1-\alpha} \mu^{\alpha-1}\right]\left\|A^{-\alpha} e\right\|_{E}= \\
& =M(M+1)\left[\frac{\operatorname{sen} \pi \alpha}{\pi \alpha}+\frac{\operatorname{sen} \pi(1-\alpha)}{\pi(1-\alpha)}\right] \mu^{\alpha-1}\left\|A^{-\alpha} e\right\|_{E} \leq \\
& \leq 2 M(M+1) \mu^{\alpha-1}\left\|A^{-\alpha} e\right\|_{E} .
\end{aligned}
$$

Como $\overline{D(A)}=E$, (2.26) segue para $0<\alpha<1$ e $K=2 M(M+1)$.

Assim, como $M>1$, temos $M<2 M(M+1)$ e $M+1<2 M(M+1)$. Logo, basta tomar $K=2 M(M+1)$ e (2.26) segue para $0 \leq \alpha \leq 1$ e $K$ constante dependendo de $M$.

\section{Teorema 2.5}

1. Suponha que $A \in \mathcal{P}(E)$ e que $e \in D\left(A^{\alpha}\right)$ para algum $\alpha, 0<\alpha \leq 1$. Então, se $e_{\epsilon}=(I+\epsilon A)^{-1} e, \epsilon>0$, temos que

$$
\begin{gathered}
\left\|e_{\epsilon}-e\right\|_{E} \leq K \epsilon^{\alpha}\left\|A^{\alpha} e\right\|_{E}, \\
\left\|A e_{\epsilon}\right\|_{E} \leq K \epsilon^{\alpha-1}\left\|A^{\alpha} e\right\|_{E}
\end{gathered}
$$

para todo $\epsilon>0$.

2. Suponha que e $\in E$ e que para algum $\alpha, 0<\alpha \leq 1$, $\|e\|_{E}<B<\infty$, existe $e_{\epsilon} \in D(A)$, para todo $\epsilon>0$ tal que

$$
\begin{gathered}
\left\|e_{\epsilon}-e\right\|_{E} \leq B \epsilon^{\alpha}, \quad \forall \epsilon>0, \\
\left\|A e_{\epsilon}\right\|_{E} \leq B \epsilon^{\alpha-1}, \quad \forall \epsilon>0 .
\end{gathered}
$$

Então $e \in D\left(A^{\beta}\right)$ para qualquer $\beta$ em $0<\beta<\alpha e$

$$
\left\|A^{\beta} e\right\|_{E} \leq M_{\alpha, \beta} B
$$

para uma constante $M_{\alpha, \beta}$ dependendo somente de $A, \alpha$ e $\beta$.

Prova. 1) Pelo Teorema 2.4

$$
\begin{aligned}
\left\|A e_{\epsilon}\right\|_{E} & =\left\|A^{1-\alpha}(1+\epsilon A)^{-1} A^{\alpha} e\right\|_{E} \leq \\
& \leq K \epsilon^{\alpha-1}\left\|A^{\alpha} e\right\|_{E}
\end{aligned}
$$


e portanto $\left\|e_{\epsilon}-e\right\|_{E}=\left\|\epsilon A e_{\epsilon}\right\|_{E} \leq K \epsilon^{\alpha}\left\|A^{\alpha} e\right\|_{E}$.

2) Para qualquer $\mu>0, \epsilon>0$

$$
\begin{aligned}
\left\|A(\mu+A)^{-1} e\right\|_{E} & \leq\left\|A(\mu+A)^{-1}\left(e-e_{\epsilon}\right)\right\|_{E}+\left\|(\mu+A)^{-1} A e_{\epsilon}\right\|_{E} \leq \\
& \leq(M+1) B \epsilon^{\alpha}+M \mu^{-1} B \epsilon^{\alpha-1} .
\end{aligned}
$$

Logo, escolhendo $\epsilon=\mu^{-1}$

$$
\left\|A(\mu+A)^{-1} e\right\|_{E} \leq B(2 M+1) \mu^{-\alpha}
$$

e claramente

$$
\left\|A(\mu+A)^{-1} e\right\|_{E} \leq(M+1)\|e\|_{E} \leq B(2 M+1) .
$$

Logo

$$
\left\|A(\mu+A)^{-1} e\right\|_{E} \leq B(2 M+1) \min \left\{1, \mu^{-\alpha}\right\} .
$$

Se $0<\beta<\alpha$ segue que $\int_{0}^{\infty}\left\|s^{\beta-1} A(s+A)^{-1} e\right\|_{E} d s<\infty$ e

$$
J_{\beta} e=\frac{\operatorname{sen} \pi \beta}{\pi} \int_{0}^{\infty} s^{\beta-1} A(s+A)^{-1} e d s
$$

é tal que $\left\|J_{\beta} e\right\|_{E} \leq M_{\alpha, \beta} B$. Mas

$$
f_{R}=\frac{\operatorname{sen} \pi \beta}{\pi} \int_{0}^{R} s^{\beta-1}(s+A)^{-1} e d s \rightarrow A^{\beta-1} e
$$

quando $R \rightarrow \infty$ e $A f_{R} \rightarrow J_{\beta}$ e quando $R \rightarrow \infty$. Como $A$ é fechado segue que $A^{\beta-1} e \in D(A)$ o que significa $e \in D\left(A^{\beta}\right)$, desde que $e=A^{-\beta}\left(A A^{\beta-1} e\right)$ e $\left\|A^{\beta} e\right\|_{E}=\left\|J_{\beta} e\right\|_{E} \leq M_{\alpha, \beta} B$.

Corolário 2.1 Se e $\in D\left(A^{\alpha}\right), \alpha>0$ e $0<\beta<\alpha$ então

$$
A^{\beta} e=\frac{\operatorname{sen} \pi \beta}{\pi} \int_{0}^{\infty} s^{\beta-1} A(s+A)^{-1} e d s .
$$

Teorema 2.6 Existe uma constante $K$ dependendo somente de $A$, tal que

$$
\left\|A^{\alpha} e\right\|_{E} \leq K\|A e\|_{E}^{\alpha}\|e\|_{E}^{1-\alpha}
$$

para $0 \leq \alpha \leq 1, e \in D(A)$.

Prova. O reultado é trivial para $\alpha=0$ e para $\alpha=1$. Como mostrado no Corolário 2.1, para $0<\alpha<1, e \in D(A)$

$$
A^{\alpha} e=\frac{\operatorname{sen} \pi \alpha}{\pi} \int_{0}^{\infty} s^{\alpha-1} A(s+A)^{-1} e d s
$$


Logo

$$
\begin{aligned}
\left\|A^{\alpha} e\right\|_{E} & \leq \frac{\operatorname{sen} \pi \alpha}{\pi}\left[\int_{0}^{\mu} s^{\alpha-1}(M+1)\|e\| d s+\int_{\mu}^{\infty} s^{\alpha-2} M\|A e\| d s\right] \leq \\
& \leq \frac{\operatorname{sen} \pi \alpha}{\pi}(M+1)\left[\frac{\mu^{\alpha}}{\alpha}\|e\|_{E}+\frac{\mu^{\alpha-1}}{1-\alpha}\|A e\|_{E}\right]
\end{aligned}
$$

para qualquer $\mu>0$. Seja $\mu=\|A e\|_{E} /\|e\|_{E}$. Então, como $0<\alpha<1$

$$
\begin{aligned}
\left\|A^{\alpha} e\right\|_{E} & \leq(M+1) \frac{\operatorname{sen} \pi \alpha}{\pi}\left[\frac{1}{\alpha}+\frac{1}{1-\alpha}\right]\|A e\|_{E}^{\alpha}\|e\|_{E}^{1-\alpha}= \\
& =(M+1)\left[\frac{\operatorname{sen} \pi \alpha}{\pi \alpha}+\frac{\operatorname{sen} \pi(1-\alpha)}{\pi(1-\alpha)}\right]\|A e\|_{E}^{\alpha}\|e\|_{E}^{1-\alpha} \leq \\
& \leq 2(M+1)\|A e\|_{E}^{\alpha}\|e\|_{E}^{1-\alpha}
\end{aligned}
$$

e o resultado segue.

Corolário 2.2 Seja $A \in \mathcal{P}(E)$ e $B: D(B) \subset E \rightarrow E$ um operador linear fechado tal que $D(B) \supset D\left(A^{\alpha}\right)$, para algum $\alpha \in(0,1)$. Então existem constantes $C, C_{1}>0$ tais que

$$
\|B e\|_{E} \leq C\left\|A^{\alpha} e\right\|_{E}, \quad e \in D\left(A^{\alpha}\right)
$$

$e$

$$
\|B e\|_{E} \leq C_{1}\left(\mu^{\alpha}\|e\|_{E}+\mu^{\alpha-1}\|A e\|_{E}\right), \quad \mu>0, e \in D(A) .
$$

Prova. Considere o operador fechado $B A^{-\alpha}$. Como $D(B) \supset D\left(A^{\alpha}\right), B A^{-\alpha}$ está definido em todo $E$ e pelo Teorema do Gráfico Fechado segue que $B A^{-\alpha} \in L(E)$. Isto e o Teorema 2.6 implicam o resultado desejado.

Teorema 2.7 Suponha que $A$ e $B$ são operadores setoriais com constante $a=0$ em $E$ com $D(A)=D(B) e \operatorname{Re} \sigma(A)>0, \operatorname{Re} \sigma(B)>0$ e para algum $\alpha \in[0,1),(A-B) A^{-\alpha} \in L(E)$. Então, para todo $\beta \in[0,1], A^{\beta} B^{-\beta}$ e $B^{\beta} A^{-\beta}$ estão em $L(E)$.

Prova. O caso $\beta=0$ é trivial. Como $\operatorname{Re} \sigma(A)>0, \operatorname{Re} \sigma(B)>0$ então $A^{-1}$ e $B^{-1}$ são limitados. Daí, como $A$ e $B$ são fechados e $D(A)=D(B)$, segue do Teorema do Gráfico Fechado que $A B^{-1} \in \mathrm{L}(E)$ e $B A^{-1} \in L(E)$, e o resultado segue para $\beta=1$. Agora, segue do Teorema 2.6 que, para $0 \leq \beta \leq 1$,

$$
\left\|A^{\beta}(\lambda+A)^{-1}\right\|_{L(E)} \leq C_{A}|\lambda|^{\beta-1}
$$

em $\Sigma_{A}=\left\{\lambda \in \mathbb{C}:|\pi-\arg \lambda| \geq \phi_{A}\right\}$, para alguma constante positiva $C_{A}$ (dependendo de $A$ ) e $\phi_{A}<\pi / 2 \mathrm{e}$

$$
\left\|B^{\beta}(\lambda+B)^{-1}\right\|_{L(E)} \leq C_{B}|\lambda|^{\beta-1}
$$


em $\Sigma_{B}=\left\{\lambda \in \mathbb{C}:|\pi-\arg \lambda| \geq \phi_{B}\right\}$, para alguma constante positiva $C_{B}$ (dependendo de $B$ ) e $\phi_{B}<\pi / 2$. Veja também que como $(A-B) A^{-\alpha} \in L(E)$, para algum $\alpha \in[0,1)$, existe $K>0$ tal que

$$
\left\|(A-B) A^{-\alpha}\right\|_{L(E)} \leq K
$$

Ainda, para $0<\beta<1$, pela Proposição 2.1

$$
A^{-\beta}=\frac{\operatorname{sen} \pi \beta}{\pi} \int_{0}^{\infty} \lambda^{-\beta}(\lambda+A)^{-1} d \lambda
$$

e

$$
B^{-\beta}=\frac{\operatorname{sen} \pi \beta}{\pi} \int_{0}^{\infty} \lambda^{-\beta}(\lambda+B)^{-1} d \lambda
$$

Logo,

$$
B^{-\beta}-A^{-\beta}=\frac{\operatorname{sen} \pi \beta}{\pi} \int_{0}^{\infty} \lambda^{-\beta}(\lambda+B)^{-1}(A-B)(\lambda+A)^{-1} d \lambda,
$$

$0<\beta<1$. Estimativas simples agora mostram que $B^{\beta} A^{-\beta}$ é limitado para $0<\beta<1$.

Por outro lado, como

$$
\left[I+A^{\alpha}(\lambda+A)^{-1}(B-A) A^{-\alpha}\right] A^{\alpha}(\lambda+B)^{-1}=A^{\alpha}(\lambda+A)^{-1},
$$

para algum $\alpha \in[0,1)$, segue que $\left\|A^{\alpha}(\lambda+B)^{-1}\right\|=O\left(|\lambda|^{\alpha-1}\right)$ quando $|\lambda| \rightarrow \infty$ e trocando $A$ por $B$ em (2.27) obtemos que $A^{\beta} B^{-\beta}$ é também limitado para $0<\beta<1$.

Corolário 2.3 Se $A$ e $B$ são como no Teorema 2.7 então $D\left(A^{\alpha}\right)=D\left(B^{\alpha}\right)$, com normas equivalentes, para $0 \leq \alpha \leq 1$.

\subsection{Potências fracionárias e semigrupos}

Agora consideramos o caso em que $A$ é setorial; isto é, $\left\{\mathbf{e}^{-A t}, t \geq 0\right\}$ é semigrupo analítico.

Teorema 2.8 Suponha que A é setorial. Logo $\left\{\mathbf{e}^{-A t} ; t \geq 0\right\}$ é um semigrupo analítico. Suponha que $\rho(A) \supset(-\infty, 0]$. Então

1. Se $t>0, \alpha \geq 0, R\left(\mathbf{e}^{-A t}\right) \subset D\left(A^{\alpha}\right) e$

$$
\left\|A^{\alpha} \mathbf{e}^{-A t}\right\|_{L(E)} \leq M_{\alpha} t^{-\alpha} \quad \text { com } \quad \alpha \mapsto M_{\alpha} \text { contínua em }[0, \infty) .
$$

2. Se $\alpha>0$, temos que $t^{\alpha} A^{\alpha} \mathbf{e}^{-A t} e \rightarrow 0$ quando $t \rightarrow 0^{+}$para cada $e \in E$.

3. $\left\|\left(\mathbf{e}^{-A t}-I\right) A^{-\alpha}\right\|_{L(E)} \leq M_{1-\alpha} \frac{t^{\alpha}}{\alpha}$ se $0<\alpha \leq 1,0 \leq t \leq 1$. 
Prova. 1) Se $t>0$, temos $R\left(\mathbf{e}^{-A t}\right) \subset D(A)$. Então segue facilmente por indução que $R\left(\mathbf{e}^{-A t}\right) \subset \bigcap_{n=1}^{\infty} D\left(A^{n}\right)$. Logo, como $D\left(A^{n}\right) \subset D\left(A^{\alpha}\right)$ para $n \geq \alpha$, temos que $R\left(\mathbf{e}^{-t A}\right) \subset D\left(A^{\alpha}\right)$.

Mostremos agora que $\left\|A^{\alpha} \mathbf{e}^{-A t}\right\|_{L(E)} \leq M_{\alpha} t^{-\alpha}, t>0, \alpha \geq 0$. Seja $n-1<\alpha \leq n$. Se $\alpha=n$, temos

$$
\left\|A^{n} \mathbf{e}^{-A t}\right\|_{L(E)}=\left\|\left(A \mathbf{e}^{-A t / n}\right)^{n}\right\|_{L(E)} \leq\left\|A \mathbf{e}^{-A t / n}\right\|_{L(E)}^{n} .
$$

Pelo Teorema 1.18, existe constante $M>0$ tal que

$$
\left\|A \mathbf{e}^{-A t}\right\|_{L(E)} \leq M t^{-1} \text { e }\left\|\mathbf{e}^{-A t}\right\|_{L(E)} \leq M
$$

Portanto,

$$
\left\|A^{n} \mathbf{e}^{-A t}\right\|_{L(E)} \leq\left(M(t / n)^{-1}\right)^{n}=M_{n} t^{-n}
$$

e

$$
\left\|A^{0} \mathbf{e}^{-A t}\right\|_{L(E)}=\left\|\mathbf{e}^{-A t}\right\|_{L(E)} \leq M
$$

Deste modo assumiremos $\alpha>0$ e $n-1<\alpha<n$. Pelo Teorema 2.3,

$$
A^{\alpha-n}=\frac{1}{\Gamma(n-\alpha)} \int_{0}^{\infty} s^{n-\alpha-1} \mathbf{e}^{-A s} d s .
$$

Logo,

$$
\begin{aligned}
\left\|A^{\alpha} \mathbf{e}^{-A t}\right\|_{L(E)} & =\left\|A^{\alpha-n} A^{n} \mathbf{e}^{-A t}\right\|_{L(E)} \leq \\
& \leq \frac{1}{\Gamma(n-\alpha)} \int_{0}^{\infty} s^{n-\alpha-1}\left\|A^{n} \mathbf{e}^{-A(t+s)}\right\|_{L(E)} d s \leq \\
& \leq \frac{M_{n}}{\Gamma(n-\alpha) t^{\alpha}}\left[\int_{0}^{1} s^{n-\alpha-1}(1+s)^{-n} d s+\int_{1}^{\infty} s^{n-\alpha-1}(1+s)^{-n} d s\right] \leq \\
& \leq \frac{M_{n}}{\Gamma(n-\alpha) t^{\alpha}}\left[\frac{1}{n-\alpha}+\frac{1}{\alpha}\right]= \\
& =\frac{M_{n}}{\Gamma(n-\alpha) t^{\alpha}} \frac{n}{\alpha(n-\alpha)}, \alpha>0 .
\end{aligned}
$$

Portanto,

$$
\left\|A^{\alpha} \mathbf{e}^{-A t}\right\|_{L(E)} \leq M_{\alpha} t^{-\alpha}, \quad \forall \alpha \geq 0, \quad \forall t>0 .
$$

Agora, como a função $\Gamma$ é contínua temos que

$$
\alpha \mapsto M_{\alpha}=\frac{M_{n}}{\Gamma(n-\alpha)} \frac{n}{\alpha(n-\alpha)} \quad(\text { onde } n \in \mathbb{N} \text { é tal que } n-1<\alpha<n \text { ) }
$$

é contínua em $[0, \infty)$.

2) Se $e \in D\left(A^{m}\right)$ para algum $m \geq \alpha>0$,

$$
t^{\alpha} A^{\alpha} \mathbf{e}^{-A t} e \stackrel{t \rightarrow 0^{+}}{\longrightarrow} 0 .
$$


Além disso, segue de 1) que

$$
\left\|t^{\alpha} A^{\alpha} \mathbf{e}^{-A t}\right\|_{L(E)} \leq M_{\alpha}
$$

para $0<t \leq 1$. Daí, como $\overline{D\left(A^{m}\right)}=E$, o resultado vale para todo $e \in E$.

3) Para todo $e \in E$, pelo Teorema 1.17, temos que

$$
\left\|\left(\mathbf{e}^{-A t}-I\right) A^{-\alpha} e\right\|_{E}=\left\|-\int_{0}^{t} A^{1-\alpha} \mathbf{e}^{-A s} e d s\right\|_{E} \leq \int_{0}^{t} M_{1-\alpha} s^{\alpha-1}\|e\|_{E} d s=M_{1-\alpha} \frac{t^{\alpha}}{\alpha}\|e\|_{E}
$$

e o resultado segue. 


\section{Capítulo 3}

\section{O problema de Cauchy}

\subsection{O problema de Cauchy não-homogêneo}

\subsubsection{Existência, unicidade e regularidade}

A seguir estudamos problemas de Cauchy (problemas de valor inicial) para equações lineares não homogêneas da forma

$$
\begin{aligned}
& \frac{d e}{d t}=A e+f(t), \quad t_{0}<t<t_{1} \\
& e\left(t_{0}\right)=e_{0} \in E
\end{aligned}
$$

onde $A$ é o gerador de um semigrupo fortemente contínuo $\left\{\mathbf{e}^{A t} ; t \geq 0\right\} \subset L(E)$ e $f:\left[t_{0}, t_{1}\right) \rightarrow E$ é contínua por partes e contínua à direita.

\section{Definição 3.1}

a) Uma solução forte de (3.1), é uma função contínua e $:\left[t_{0}, t_{1}\right) \rightarrow E$ tal que e $\left(t_{0}\right)=e_{0} e$ para $t_{0}<t<t_{1}, e(t) \in D(A)$,

$$
\frac{d^{+}}{d t} e(t)=\lim _{h \rightarrow 0^{+}} \frac{e(t+h)-e(t)}{h}
$$

existe, (3.1) vale com $\frac{d}{d t} e(t)$ substituido por $\frac{d^{+}}{d t} e(t)$ e $t \mapsto \frac{d^{+}}{d t} e(t)$ é contínua onde $f$ é contínua. Note que se $t \mapsto f(t)$ é uma função contínua e $t \mapsto e(t)$ é uma solução forte de (3.1) então $t \mapsto e(t)$ é contínuamente diferenciável e (3.1) se verifica para cada $t \in\left(t_{0}, t_{1}\right)$.

b) Uma solução fraca de (3.1) em $\left[t_{0}, t_{1}\right)$ é uma função contínua e : $\left[t_{0}, t_{1}\right) \rightarrow E$ tal que $e\left(t_{0}\right)=e_{0} \in E$ e para todo $e^{*} \in D\left(A^{*}\right), t \mapsto\left\langle e^{*}, e(t)\right\rangle$ tem derivada à direita $e$

$$
\frac{d^{+}}{d t}\left\langle e^{*}, e(t)\right\rangle=\left\langle A^{*} e^{*}, e(t)\right\rangle+\left\langle e^{*}, f(t)\right\rangle, \quad t_{0}<t<t_{1} .
$$


Note que se $t \mapsto\left\langle e^{*}, f(t)\right\rangle$ é uma função contínua e $t \mapsto e(t)$ é uma solução fraca de (3.1) então $t \mapsto\left\langle e^{*}, e(t)\right\rangle$ é contínuamente diferenciável e (3.2) se verifica com $\frac{d^{+}}{d t}$ substituído por $\frac{d}{d t}$.

Definição 3.2 Um subconjunto $S^{*} \subset E^{*}$ é dito total se: $e \in E,\left\langle e^{*}, e\right\rangle=0, \forall e^{*} \in S^{*}$ implica $e=0$.

$O$ anulador $S^{\perp} \subset E^{*}$ de um subconjunto $S \subset E$ é o conjunto de todos os elementos $e^{*} \in E^{*}$ tais que $\left\langle e^{*}, e\right\rangle=0, \forall e \in S$. Sabemos que se $S \subset E$ é um espaço vetorial então $\left(S^{\perp}\right)^{\perp}=\bar{S}$ (veja [4]).

Lema 3.1 Se $A: D(A) \subset E \rightarrow E$ é fechado e densamente definido então $D\left(A^{*}\right)$ é total.

Prova. Seja $e \in E$ tal que $\left\langle e^{*}, e\right\rangle=0$ para todo $e^{*} \in D\left(A^{*}\right)$. Queremos mostrar que $e=0$. Veja que o gráfico de $A^{*}, G\left(A^{*}\right)=\left\{\left(e^{*}, A^{*} e^{*}\right): e^{*} \in D\left(A^{*}\right)\right\}$, é o anulador em $E^{*} \times E^{*}$ de $\Gamma=\{(-A e, e): e \in D(A)\}$; isto é, $G\left(A^{*}\right)=\Gamma^{\perp}$. Note que $G\left(A^{*}\right)$ também anula $(e, 0)$ e então $(e, 0) \in G\left(A^{*}\right)^{\perp}=\Gamma$ e portanto $e=0$.

O teorema a seguir nos dá formas de manuseio mais simples para as soluções fracas e estabelece algumas relações importantes entre soluções fracas e fortes.

\section{Teorema 3.1}

1. Se e $:\left[t_{0}, t_{1}\right) \rightarrow E$ é uma solução forte de (3.1) então é também uma solução fraca de (3.1).

2. Se e $:\left[t_{0}, t_{1}\right) \rightarrow E$ é uma solução fraca de (3.1), então

$$
e(t)=\mathbf{e}^{A\left(t-t_{0}\right)} e_{0}+\int_{t_{0}}^{t} \mathbf{e}^{A(t-s)} f(s) d s, \quad t_{0} \leq t<t_{1} .
$$

Em particular, existe uma única solução fraca de (3.1).

3. Se e $:\left[t_{0}, t_{1}\right) \rightarrow E$ é definido por (3.3), então e $:\left[t_{0}, t_{1}\right) \rightarrow E$ é uma solução fraca de (3.1).

4. Se e $:\left[t_{0}, t_{1}\right) \rightarrow E$ é uma solução fraca de (3.1) e para algum $t \in\left(t_{0}, t_{1}\right)$ ou e $(t) \in D(A)$ ou $\frac{d^{+}}{d t} e(t)$ existe, então ambos são verdadeiros e para este instante

$$
\frac{d^{+}}{d t} e(t)=A e(t)+f(t)
$$

Prova. A afirmativa 1) é trivial. Provaremos 3) e a unicidade de soluções fracas o que implicará 2).

Prova de 3). Defina $e:\left[t_{0}, t_{1}\right) \rightarrow E$ por $(\overline{3.3})$ e seja $e^{*} \in D\left(A^{*}\right)$. Para qualquer $e \in E$ $t \mapsto\left\langle e^{*}, \mathbf{e}^{A t} e\right\rangle$ é diferenciável com derivada $\left\langle A^{*} e^{*}, \mathbf{e}^{A t} e\right\rangle$, pois

$$
\left\langle e^{*}, \mathbf{e}^{A t} e\right\rangle-\left\langle e^{*}, e\right\rangle=\int_{0}^{t}\left\langle A^{*} e^{*}, \mathbf{e}^{A s} e\right\rangle d s
$$


para $e \in D(A)$ e por continuidade para todo $e \in E$. Usando isto calculamos $\frac{d^{+}}{d t}\left\langle e^{*}, e(t)\right\rangle$ e vemos que $e:\left[t_{0}, t_{1}\right) \rightarrow E$ é uma solução fraca. De fato,

$$
\begin{aligned}
& \left\langle e^{*}, \int_{t_{0}}^{t+h} \mathbf{e}^{A(t+h-s)} f(s) d s\right\rangle-\left\langle e^{*}, \int_{t_{0}}^{t} \mathbf{e}^{A(t-s)} f(s) d s\right\rangle= \\
& =\left\langle e^{*}, \int_{t}^{t+h} \mathbf{e}^{A(t+h-s)} f(s) d s\right\rangle-\left\langle e^{*},\left(\mathbf{e}^{A h}-I\right) \int_{t_{0}}^{t} \mathbf{e}^{A(t-s)} f(s) d s\right\rangle= \\
& =h\left[\left\langle e^{*}, f(t)\right\rangle+\left\langle A^{*} e^{*}, \int_{t_{0}}^{t} \mathbf{e}^{A(t-s)} f(s) d s\right\rangle\right]+o(h)
\end{aligned}
$$

onde na última passagem utilizamos que $A^{*}$ é o gerador infinitesimal do semigrupo fortemente contínuo $\left\{T^{\odot}(t)=\left.(T(t))^{*}\right|_{Y}: t \geq 0\right\}$ em $L(Y), Y={\overline{D\left(A^{*}\right)}}^{E^{*}}$ (veja Teorema 1.14).

Prova da unicidade em 2). Se existem duas soluções de (3.1), a diferença entre elas $u:\left[t_{0}, t_{1}\right) \rightarrow E$ é uma função contínua com $u\left(t_{0}\right)=0$ e $\frac{d^{+}}{d t}\left\langle e^{*}, u(t)\right\rangle=\left\langle A^{*} e^{*}, u(t)\right\rangle, t_{0} \leq t<t_{1}$ e $e^{*} \in D\left(A^{*}\right)$. É conveniente trabalhar com uma função $C^{1}$. Logo seja $U(t)=\int_{t_{0}}^{t} u(s) d s$, então

$$
\left\langle e^{*}, u(t)\right\rangle=\int_{t_{0}}^{t}\left\langle A^{*} e^{*}, u(s)\right\rangle d s \quad e\left\langle e^{*}, \frac{d}{d t} U(t)\right\rangle=\left\langle A^{*} e^{*}, U(t)\right\rangle .
$$

Agora observe que $\left(\mathbf{e}^{A t}\right)^{*}\left(D\left(A^{*}\right)\right) \subset D\left(A^{*}\right)$ para $t \geq 0$, já que $\left\langle\left(\mathbf{e}^{A t}\right)^{*} e^{*}, A e\right\rangle=\left\langle A^{*} e^{*}, \mathbf{e}^{A t} e\right\rangle$ para $e^{*} \in D\left(A^{*}\right), e \in D(A)$. Logo, para qualquer $t^{*} \in\left(t_{0}, t_{1}\right)$

$$
\left\langle e^{*}, \mathbf{e}^{A\left(t^{*}-t\right)} \frac{d}{d t} U(t)\right\rangle=\left\langle A^{*} e^{*}, \mathbf{e}^{A\left(t^{*}-t\right)} U(t)\right\rangle \text { e } \frac{d}{d t}\left\langle e^{*}, \mathbf{e}^{A\left(t^{*}-t\right)} U(t)\right\rangle=0
$$

para $t_{0} \leq t \leq t^{*}$.

Como $U\left(t_{0}\right)=0,\left\langle e^{*}, U\left(t^{*}\right)\right\rangle=0$ para todo $e^{*} \in D\left(A^{*}\right)$. Portanto, como $D\left(A^{*}\right)$ é total (Lema 3.1), $U\left(t^{*}\right)=0$ e então $u(s)=0$ para $t_{0} \leq s \leq t_{1}$.

Prova de 4). Se $e(\cdot)$ é uma solução fraca, dada por (3.3), então para $t_{0} \leq t<t+h<t_{1}$

$$
\frac{e(t+h)-e(t)}{h}=\frac{1}{h} \int_{t}^{t+h} \mathbf{e}^{A(t+h-s)} f(s) d s+\frac{1}{h}\left(\mathbf{e}^{A h}-I\right) e(t) .
$$

O termo do meio converge para $f\left(t^{+}\right)=f(t)$ quando $h \rightarrow 0^{+}$. Logo se $e(t) \in D(A)$, então $\frac{d^{+}}{d t} e(t)$ existe e, ainda,

$$
\frac{d^{+}}{d t} e(t)=A e(t)+f(t)
$$

Da mesma forma, se $\frac{d^{+}}{d t} e(t)$ existe, então $e(t) \in D(A)$ e

$$
\frac{d^{+}}{d t} e(t)=A e(t)+f(t)
$$

o que termina a prova.

A seguir damos condições simples que asseguram a diferenciabilidade de uma solução fraca. 
Teorema 3.2 Admita que A e $f$ são como antes e que $e:\left[t_{0}, t_{1}\right) \rightarrow$ E é uma solução fraca de (3.1). Se $e_{0} \in D(A)$ e ou

1. $f(t) \in D(A)$ com $t \mapsto A f(t) \in E$ contínua à direita em $\left[t_{0}, t_{1}\right)$ ou

2. $\frac{d^{+}}{d t} f(t)=\dot{f}(t)$ existe e é contínua à direita em $\left[t_{0}, t_{1}\right)$

então $\frac{d^{+}}{d t} e(t)$ existe, $e(t) \in D(A)$ e e $:\left[t_{0}, t_{1}\right) \rightarrow E$ é uma solução forte.

Prova. Seja $u(t)=\int_{t_{0}}^{t} \mathbf{e}^{A(t-s)} f(s) d s, t \in\left[t_{0}, t_{1}\right)$. Logo, $e(t)=\mathbf{e}^{A\left(t-t_{0}\right)} e_{0}+u(t)$ e como $e_{0} \in D(A)$, $t \mapsto \mathbf{e}^{A\left(t-t_{0}\right)} e_{0}$ é $C^{1}, t \in\left[t_{0}, t_{1}\right)$. Se $t_{0} \leq t<t+h<t_{1}$,

$$
\frac{u(t+h)-u(t)}{h}=\frac{1}{h} \int_{t}^{t+h} \mathbf{e}^{A(t+h-s)} f(s) d s+\int_{t_{0}}^{t} \mathbf{e}^{A(t-s)} \frac{\mathbf{e}^{A h}-I}{h} f(s) d s
$$

e

$$
\frac{u(t+h)-u(t)}{h}=\frac{1}{h} \int_{t_{0}}^{t_{0}+h} \mathbf{e}^{A(t+h-s)} f(s) d s+\int_{t_{0}}^{t} \mathbf{e}^{A(t-s)} \frac{f(s+h)-f(s)}{h} d s
$$

e usando as primeira e segunda expressões nos casos 1. e 2. respectivamente, vemos que

$$
\left.\frac{d^{+}}{d t} u(t)=f(t)+\int_{t_{0}}^{t} \mathbf{e}^{A(t-s)} A f(s) d s, \quad \text { (no caso } 1 .\right)
$$

e

$$
\frac{d^{+}}{d t} u(t)=\mathbf{e}^{A\left(t-t_{0}\right)} f\left(t_{0}\right)+\int_{t_{0}}^{t} \mathbf{e}^{A(t-s)} \dot{f}(s) d s, \quad \text { (no caso 2.). }
$$

Pelo Teorema $3.1(4), u(t) \in D(A)$ e a prova está completa.

\subsection{O problema de Cauchy semilinear - o caso parabólico}

Nesta seção consideramos problemas de Cauchy da forma

$$
\begin{aligned}
& \frac{d}{d t} e=-A e+f(t, e) \\
& e\left(t_{0}\right)=e_{0}
\end{aligned}
$$

onde $A: D(A) \subset E \rightarrow E$ é um operador setorial positivo.

Recorde que se o operador $A$ ou (uma translação deste) é de tipo posivivo podemos definir as suas potências fracionárias $A^{\alpha}, \alpha \in \mathbb{R}$. Denote por $E^{\alpha}$ o domínio de $A^{\alpha}$ dotado com a norma do gráfico. É claro que $E^{\alpha}$ é um espaço de Banach e que a restrição de $\left\{\mathbf{e}^{-A t} ; t \geq 0\right\}$ a $E^{\alpha}$ é um semigrupo fortemente contínuo em $E^{\alpha}$, visto que

$$
A^{\alpha} \mathbf{e}^{-A t} e=\mathbf{e}^{-A t} A^{\alpha} e, \quad \forall t \geq 0, e \in E^{\alpha} .
$$


Com as informações que temos até agora podemos obter teoremas de existência e regularidade apropriados para o caso parabólico. O que faremos aqui é mostrar que, sob condições bastante razoáveis, toda solução de (3.4) com dados iniciais em algum $E^{\alpha}, \alpha<1$ são soluções fortes. Também estaremos lidando com uma classe mais ampla de não linearidades $f$, permitindo que dependa de $A^{\alpha} e$, por exemplo.

Definição 3.3 Seja E um espaço de Banach, $A: D(A) \subset E \rightarrow E$ um operador setorial e escolha $a \geq 0$ (se necessário) tal que $(-\infty, 0] \subset \rho(A+a)$. Seja $0 \leq \alpha \leq 1$ e $E^{\alpha}=D\left((A+a)^{\alpha}\right)$ com a norma do gráfico $\|\cdot\|_{\alpha}=\left\|(A+a)^{\alpha} \cdot\right\|_{E}$. Seja $U \subset \mathbb{R} \times E^{\alpha}$ um aberto e $f: U \rightarrow E$ contínua.

a) Uma solução (solução forte) de (3.4) em $\left[t_{0}, t_{1}\right)$ é uma função contínua e $:\left[t_{0}, t_{1}\right) \rightarrow E^{\alpha}$ tal que $e:\left(t_{0}, t_{1}\right) \rightarrow E^{\alpha}$ é continuamente diferenciável, $e\left(t_{0}\right)=e_{0},(t, e(t)) \in U$, $e(t) \in D(A)$ e (3.4) vale, $t_{0}<t<t_{1}$.

b) Uma solução fraca de (3.4) em $\left[t_{0}, t_{1}\right)$ é uma função contínua e $:\left[t_{0}, t_{1}\right) \rightarrow E^{\alpha}$ tal que $e\left(t_{0}\right)=e_{0},(t, e(t)) \in U$, para todo $t \in\left(t_{0}, t_{1}\right)$ e para cada $e^{*} \in D\left(A^{*}\right), t \mapsto\left\langle e^{*}, e(t)\right\rangle$ é differenciável e

$$
\frac{d}{d t}\left\langle e^{*}, e(t)\right\rangle=\left\langle A^{*} e^{*}, e(t)\right\rangle+\left\langle e^{*}, f(t, e(t))\right\rangle, \quad t_{0}<t<t_{1} .
$$

Em nossos resultados consideraremos sem perda de generalidade que $A$ é setorial com constante $a=0$ (veja definição 1.11).

Com isto temos o seguinte teorema:

\section{Teorema 3.3}

1. Se e $:\left[t_{0}, t_{1}\right) \rightarrow E^{\alpha}$ é uma solução forte de (3.4) então é também uma solução fraca de (3.4).

2. Uma solução fraca e $:\left[t_{0}, t_{1}\right) \rightarrow E^{\alpha}$ de (3.4) é também uma solução forte se, e somente se, é continuamente diferenciável em $\left(t_{0}, t_{1}\right)$ se, e somente se, $e(t) \in D(A)$ com $t \mapsto A e(t)$ contínua em $\left(t_{0}, t_{1}\right)$.

3. Se e $:\left[t_{0}, t_{1}\right) \rightarrow E^{\alpha}$ é uma solução fraca de (3.4), então

$$
e(t)=\mathbf{e}^{-A\left(t-t_{0}\right)} e_{0}+\int_{t_{0}}^{t} \mathbf{e}^{-A(t-s)} f(s, e(s)) d s, \quad t_{0} \leq t<t_{1} .
$$


4. Se e $:\left[t_{0}, t_{1}\right) \rightarrow E^{\alpha}$ é contínua com $(t, e(t)) \in U, t_{0} \leq t<t_{1}$ e satisfaz (3.6), então $e:\left[t_{0}, t_{1}\right) \rightarrow E^{\alpha}$ é uma solução fraca de (3.4).

Prova. As afirmativas do teorema seguem imediatamente do Teorema 3.1 uma vez que $t \mapsto$ $f(t, e(t)):\left[t_{0}, t_{1}\right) \rightarrow E$ é uma função contínua.

O teorema a seguir mostra que em alguns casos o conceito de solução fraca não se faz necessário para (3.4).

Teorema 3.4 Suponha que E, A, $\alpha, U$ e $f$ são como na Definição (3.3) e admita que $f$ é localmente Hölder contínua; isto é, qualquer ponto de $U$ tem uma vizinhança $V \subset U$ e constantes $K, \eta>0$, tais que quando $\left(\tau_{1}, e_{1}\right)$ e $\left(\tau_{2}, e_{1}\right)$ estão em $V$

$$
\left\|f\left(\tau_{1}, e_{1}\right)-f\left(\tau_{2}, e_{2}\right)\right\|_{E} \leq K\left(\left|\tau_{1}-\tau_{2}\right|^{\eta}+\left\|e_{1}-e_{2}\right\|_{E^{\alpha}}^{\eta}\right) .
$$

Se e $:\left[t_{0}, t_{1}\right] \rightarrow E^{\alpha}$ é contínua, $(t, e(t)) \in U, t_{0} \leq t \leq t_{1}, e$

$$
e(t)=\mathbf{e}^{-A\left(t-t_{0}\right)} e\left(t_{0}\right)+\int_{t_{0}}^{t} \mathbf{e}^{-A(t-s)} f(s, e(s)) d s, \quad t_{0} \leq t \leq t_{1},
$$

então e(t) é uma solução forte de (3.4).

Prova. Primeiramente mostraremos que $e:\left(t_{0}, t_{1}\right] \rightarrow E^{\alpha}$ é Hölder contínua. Como $\{(t, e(t))$ : $\left.t_{0} \leq t \leq t_{1}\right\}$ é um subconjunto compacto de $U$, existe $B$ tal que $\sup _{t_{0} \leq t \leq t_{1}} \| f\left(t, e(t) \|_{E} \leq B\right.$. Então para $t_{0}<t \leq t+h \leq t_{1}$,

$$
\begin{aligned}
e(t+h)-e(t) & =\left(\mathbf{e}^{-A h}-I\right)\left[\mathbf{e}^{-A\left(t-t_{0}\right)} e\left(t_{0}\right)+\int_{t_{0}}^{t} \mathbf{e}^{-A(t-s)} f(s, e(s)) d s\right]+ \\
& +\int_{t}^{t+h} \mathbf{e}^{-A(t+h-s)} f(s, e(s)) d s .
\end{aligned}
$$

Logo, se $0<\theta<1-\alpha$,

$$
\begin{aligned}
\|e(t+h)-e(t)\|_{\alpha} & \leq M_{1-\theta} M_{\theta} \frac{h^{\theta}}{\theta}\left(t-t_{0}\right)^{-\theta}\left\|e\left(t_{0}\right)\right\|_{\alpha}+M_{1-\theta} M_{\alpha+\theta} \frac{h^{\theta}}{\theta} \int_{t_{0}}^{t}(t-s)^{-\alpha-\theta} B d s+ \\
& +M_{\alpha} \int_{t}^{t+h}(t+h-s)^{-\alpha} B d s=\mathrm{O}\left(h^{\theta}\left(t-t_{0}\right)^{-\theta}\right)
\end{aligned}
$$

onde $M_{1-\theta}, M_{\theta}, M_{\alpha+\theta}$ e $M_{\alpha}$ são dados pelo Teorema 2.8. Segue que $t \mapsto f(t, e(t)) \equiv g(t)$ é contínua em $\left[t_{0}, t_{1}\right]$ e satisfaz uma condição de Hölder

$$
\|g(t+h)-g(t)\|_{E} \leq K\left(t-t_{0}\right)^{-\delta} h^{\delta}, \quad t_{0}<t \leq t+h \leq t_{1},
$$

para alguma escolha de $K, \delta>0$ (e $0<\delta<1-\alpha$, sem perda de generalidade). É suficiente provar que

$$
G(t)=\int_{t_{0}}^{t} \mathbf{e}^{-A(t-s)} g(s) d s
$$


toma valores em $D(A)$ com $t \rightarrow A G(t)$ contínua em $\left(t_{0}, t_{1}\right]$ e portanto mostraremos que $h^{-1}\left(\mathbf{e}^{-A h}-I\right) G(t)$ converge quando $h \rightarrow 0^{+}$, uniformemente para $t_{0}^{*} \leq t \leq t_{1}$, qualquer que seja $t_{0}^{*}>t_{0}$. Agora

$$
\begin{aligned}
h^{-1}\left(\mathbf{e}^{-A h}-I\right) G(t) & =\int_{t_{0}}^{t} h^{-1}\left(\mathbf{e}^{-A h}-I\right) \mathbf{e}^{-A(t-s)}[g(s)-g(t)] d s \\
& +h^{-1} \int_{t_{0}}^{t_{0}+h} \mathbf{e}^{-A(t+h-s)} g(t) d s-h^{-1} \int_{t-h}^{t} \mathbf{e}^{-A(t-s)} g(t) d s,
\end{aligned}
$$

onde os dois últimos termos são claramente uniformemente convergentes em $t$. Para o outro termo, primeiramente note que

$$
\int_{t_{0}}^{t}\left\|A \mathbf{e}^{-A(t-s)}\right\|_{L(E)}\|g(t)-g(s)\|_{E} d s<\infty
$$

da condição de Hölder, e como $\frac{d}{d t} \mathbf{e}^{-A t}=A \mathbf{e}^{-A t}, t \geq 0$,

$$
\begin{aligned}
& \left\|h^{-1}\left(\mathbf{e}^{-A h}-I\right) G(t)-\left(-\int_{t_{0}}^{t} A \mathbf{e}^{-A(t-s)}[g(s)-g(t)] d s+\mathbf{e}^{-A\left(t-t_{0}\right)} g(t)-g(t)\right)\right\|_{E}= \\
& =\left\|\int_{t_{0}}^{t} h^{-1} \int_{0}^{h}\left(I-\mathbf{e}^{-A \sigma}\right) d \sigma A \mathbf{e}^{-A(t-s)}(g(s)-g(t)) d s\right\|_{E} \leq \\
& \leq \int_{t_{0}}^{t} M h^{\epsilon}(t-s)^{-1-\epsilon} K\left(s-t_{0}\right)^{-\delta}(t-s)^{\delta} d s \stackrel{h \rightarrow 0^{+}}{\longrightarrow} 0, \quad 0<\epsilon<\delta
\end{aligned}
$$

uniformemente para $t_{0}^{*} \leq t \leq t_{1}$.

Portanto, $h^{-1}\left(\mathbf{e}^{-A h}-I\right) G(t) \rightarrow-\int_{t_{0}}^{t} A \mathbf{e}^{-A(t-s)}(g(s)-g(t)) d s+\mathbf{e}^{-A\left(t-t_{0}\right)} g(t)-g(t)$ quando $h \rightarrow 0^{+}$uniformemente em $\left[t_{0}^{*}, t_{1}\right]$, e a prova está completa.

As integrais impróprias que aparecem nas estimativas obtidas através da fórmula da variação das constantes no caso parabólico tornam necessária a obtenção de uma desigualdade de Gronwall onde as funções conhecidas possuem singularidades. Uma dessas desigualdades é obtida no lema a seguir (veja [14]).

Lema 3.2 (Desigualdade de Gronwall) Suponha que $a, b \geq 0,0 \leq \alpha, \beta<1$ e $u:[0, T] \rightarrow \mathbb{R}$ é integrável com

$$
0 \leq u(t) \leq a t^{-\alpha}+b \int_{0}^{t}(t-s)^{-\beta} u(s) d s
$$

quase sempre em $(0, T)$. Então, existe uma constante $K$ dependendo somente de $b, \beta, T$ tal que

$$
u(t) \leq \frac{K}{1-\alpha} a t^{-\alpha}
$$

quase sempre em $(0, T)$. 
Prova. Defina a transformação $B: \phi \mapsto\left\{b \int_{0}^{t}(t-s)^{-\beta} \phi(s) d s, 0 \leq t \leq T\right\}$. Então, a desigualdade (3.7) pode ser escrita na forma

$$
0 \leq u(t) \leq u_{0}(t)+B u(t)
$$

onde $u_{0}(t)=a t^{-\alpha}$. É claro que $B$ preserva ordem e portanto

$$
0 \leq u(t) \leq u_{0}(t)+B u(t) \leq u_{0}(t)+B u_{0}(t)+B^{2} u(t) \leq \cdots \leq \sum_{k=0}^{m-1} B^{k} u_{0}(t)+B^{m} u(t)
$$

para $m=1,2,3, \cdots$. Vamos mostrar que a série $\sum_{n=0}^{\infty} B^{n} u_{0}(t)$ converge e que $B^{n} u(t) \rightarrow 0$ quando $n \rightarrow \infty$ e depois obter a estimativa

$$
\sum_{n=0}^{\infty} B^{n} u_{0}(t) \leq \frac{K}{1-\alpha} a t^{-\alpha}
$$

Mostremos que

$$
B^{n} \phi(t)=\frac{(b \Gamma(1-\beta))^{n}}{\Gamma(n(1-\beta))} \int_{0}^{t}(t-s)^{n(1-\beta)-1} \phi(s) d s, \quad \forall n \in \mathbb{N} .
$$

O caso $n=2$ segue facilmente. Agora suponha, por indução, que

$$
B^{n-1} \phi(t)=\int_{0}^{t} \frac{(b \Gamma(1-\beta))^{n-1}}{\Gamma((n-1)(1-\beta))}(s-\theta)^{(n-1)(1-\beta)-1} \phi(s) d s \quad n \geq 2,
$$

então obtemos

$$
\begin{aligned}
& B\left(B^{n-1} \phi\right)(t)=b \int_{0}^{t}(t-s)^{-\beta}\left[\int_{0}^{s} \frac{(b \Gamma(1-\beta))^{n-1}}{\Gamma((n-1)(1-\beta))}(s-\theta)^{(n-1)(1-\beta)-1} \phi(\theta) d \theta\right] d s= \\
& =\frac{b^{n} \Gamma(1-\beta)^{n-1}}{\Gamma((n-1)(1-\beta))} \int_{0}^{t} \phi(\theta)\left[\int_{\theta}^{t}(t-s)^{-\beta}(s-\theta)^{(n-1)(1-\beta)-1} d s\right] d \theta= \\
& =\frac{b^{n} \Gamma(1-\beta)^{n-1}}{\Gamma((n-1)(1-\beta))} \int_{0}^{t} \phi(\theta)\left[\int_{0}^{t-\theta}(t-\theta-u)^{-\beta} u^{(n-1)(1-\beta)-1} d u\right] d \theta= \\
& =\frac{b^{n} \Gamma(1-\beta)^{n-1}}{\Gamma((n-1)(1-\beta))} \int_{0}^{t} \phi(\theta)(t-\theta)^{n(1-\beta)-1} \int_{0}^{1}(1-z)^{-\beta} z^{(n-1)(1-\beta)-1} d z d \theta= \\
& =\frac{b^{n} \Gamma(1-\beta)^{n-1}}{\Gamma((n-1)(1-\beta))} \mathbf{B}(1-\beta,(n-1)(1-\beta)) \int_{0}^{t} \phi(\theta)(t-\theta)^{n(1-\beta)-1} d \theta= \\
& =\frac{b^{n} \Gamma(1-\beta)^{n}}{\Gamma(n(1-\beta))} \int_{0}^{t} \phi(\theta)(t-\theta)^{n(1-\beta)-1} d \theta,
\end{aligned}
$$


onde utilizamos que $\mathbf{B}(x, y)=\int_{0}^{1} z^{x-1}(1-z)^{y-1} d z=\frac{\Gamma(x) \Gamma(y)}{\Gamma(x+y)}$.

Agora vamos estudar a convergência da série

$$
S_{\beta}(z)=\sum_{n=0}^{\infty} \frac{z^{n}}{\Gamma(n(1-\beta))} .
$$

Note que se $a_{n}=\Gamma(n(1-\beta))^{-1}$ temos que

$$
\begin{aligned}
\frac{a_{n+1}}{a_{n}} & =\frac{\Gamma(n(1-\beta))}{\Gamma((n+1)(1-\beta))}=\frac{\mathbf{B}(1-\beta, n(1-\beta))}{\Gamma(1-\beta)}= \\
& =\frac{1}{\Gamma(1-\beta)} \int_{0}^{1} t^{-\beta}(1-t)^{n(1-\beta)-1} d t \rightarrow 0
\end{aligned}
$$

quando $n \rightarrow \infty$, pelo Teorema da Convergência Dominada de Lebesgue.

Isto mostra que $B^{n} \phi(t) \rightarrow 0$ quando $n \rightarrow \infty$ e então

$$
u(t) \leq \sum_{n=0}^{\infty} B^{n} u_{0}(t) .
$$

Resta encontrar uma estimativa para a série acima. Note que

$$
\begin{aligned}
B^{n} u_{0}(t) & =a \frac{b^{n} \Gamma(1-\beta)^{n}}{\Gamma(n(1-\beta))} \int_{0}^{t}(t-s)^{n(1-\beta)-1} s^{-\alpha} d s= \\
& =a t^{-\alpha} \frac{z^{n(1-\beta)}}{\Gamma(n(1-\beta))} \mathbf{B}(1-\alpha, n(1-\beta)) \leq \\
& \leq \frac{a}{1-\alpha} t^{-\alpha} z^{n(1-\beta)} \Gamma(n(1-\beta))^{-1}
\end{aligned}
$$

onde $z=t(b \Gamma(1-\beta))^{1 /(1-\beta)}$. Defina, para $z \geq 0$,

$$
E_{\beta}(z):=\sum_{n=0}^{\infty} \frac{z^{n(1-\beta)}}{\Gamma(n(1-\beta)+1)} .
$$

A convergência da série acima pode ser garantida como antes. Além disso, pode ser provado (veja [7, página 38) que $E_{\beta}(z) \leq c \mathbf{e}^{z}$, para algum $c>0$.

Escolha um número natural $k$ tal que $k(1-\beta)>1$, então

$$
\begin{aligned}
S_{\beta}\left(z^{1-\beta}\right) & \leq \sum_{j=0}^{k-1} \frac{z^{j(1-\beta)}}{\Gamma(j(1-\beta))}+\frac{z^{k(1-\beta)}}{\Gamma(k(1-\beta))} \sum_{n=0}^{\infty} \frac{z^{n(1-\beta)}}{\Gamma(n(1-\beta)+1)} \leq \\
& \leq \sum_{j=0}^{k-1} \frac{z^{j(1-\beta)}}{\Gamma(j(1-\beta))}+c \frac{z^{k(1-\beta)}}{\Gamma(k(1-\beta))} \mathbf{e}^{z}
\end{aligned}
$$


para algum $c>0$. Assim, de (3.8), (3.9) e (3.10), obtemos que

$$
\begin{aligned}
u(t) & \leq \frac{a}{1-\alpha} t^{-\alpha}\left[\sum_{j=0}^{k-1} \frac{t^{j(1-\beta)}(b \Gamma(1-\beta))^{j}}{\Gamma(j(1-\beta))}\right. \\
& \left.+c(b \Gamma(1-\beta))^{k} \frac{t^{k(1-\beta)}}{\Gamma(k(1-\beta))} \mathbf{e}^{t(b \Gamma(1-\beta))^{1 /(1-\beta)}}\right]
\end{aligned}
$$

e o resultado segue.

Teorema 3.5 Suponha que E é um espaço de Banach, $A$ é um operador setorial em E, $0 \leq \alpha<1, E^{\alpha}$ é definido como anteriormente e $U$ é um subconjunto aberto de $\mathbb{R} \times E^{\alpha}$. Suponha que $f: U \rightarrow E$ é localmente Hölder contínua e localmente Lipschitz contínua em seu segundo argumento, isto é, dado $\left(t_{0}, e_{0}\right) \in U$ existem $\delta>0$ e L tais que

$$
\left\|f\left(t, e_{1}\right)-f\left(s, e_{2}\right)\right\|_{E} \leq C\left(|t-s|^{\theta}+\left\|e_{1}-e_{2}\right\|_{E^{\alpha}}\right)
$$

para $\operatorname{algum} \theta>0 e$

$$
\left\|f\left(t, e_{1}\right)-f\left(t, e_{2}\right)\right\|_{E} \leq L\left\|e_{1}-e_{2}\right\|_{E^{\alpha}}
$$

quando $\left|t-t_{0}\right| \leq \delta$ e $\left\|e_{i}-e_{0}\right\|_{E^{\alpha}} \leq \delta, i=1,2$, respectivamente. Dado qualquer $\left(t_{0}, e_{0}\right) \in U$, existe uma única solução $e:\left[t_{0}, t_{1}\right) \rightarrow E^{\alpha}$ de (3.4) definida em um intervalo maximal. Adicionalmente, se $e_{0} \in D(A)$ a derivada é contínua quando $t \rightarrow t_{0}^{+}$. Se $t_{1}<\infty$ então, quando $t \rightarrow t_{1}$ ou $(t, e(t))$ tende para algum ponto de $\partial U$ ou

$$
\frac{\|f(t, e(t))\|_{E}}{1+\|e(t)\|_{E^{\alpha}}}
$$

é ilimitada (ou ambos).

Prova. Primeiramente provamos a existência local obtendo que a transformação

$$
G(e)(t)=\mathbf{e}^{-A\left(t-t_{0}\right)} e_{0}+\int_{t_{0}}^{t} \mathbf{e}^{-A(t-s)} f(s, e(s)) d s, \quad t_{0} \leq t \leq t_{0}+T
$$

é uma contração em uma bola fechada $B_{r} \subset C\left(\left[t_{0}, t_{0}+T\right], E^{\alpha}\right)$, quando $e:\left[t_{0}, t_{0}+T\right] \rightarrow E^{\alpha}$ é contínua com $\sup _{t_{0} \leq t \leq t_{0}+T}\left\|e(t)-e_{0}\right\|_{E^{\alpha}} \leq r$; isto é, $e(\cdot) \in B_{r}$. Escolhemos $r, T>0$ pequenos de 
forma que para $t \in\left[t_{0}, t_{0}+T\right]$ e $\left\|e_{i}-e_{0}\right\|_{E_{\alpha}} \leq r$, temos $\left(t, e_{i}\right) \in U,\left\|f\left(t, e_{i}\right)\right\|_{E} \leq B, i=1,2 \mathrm{e}$

$$
\begin{aligned}
& \left\|f\left(t, e_{1}\right)-f\left(t, e_{2}\right)\right\|_{E} \leq L\left\|e_{1}-e_{2}\right\|_{E^{\alpha}}, \quad 0 \leq t \leq T ; \\
& \left\|\mathbf{e}^{-A s} z\right\|_{E^{\alpha}} \leq M\|z\|_{E^{\alpha}}, \quad 0 \leq s \leq T ; \\
& \left\|\mathbf{e}^{-A s} z\right\|_{E^{\alpha}} \leq M s^{-\alpha}\|z\|_{E}, \quad 0<s \leq T ; \\
& \left\|\mathbf{e}^{-A s} e_{0}-e_{0}\right\|_{E^{\alpha}} \leq r / 2, \quad 0 \leq s \leq T ; \\
& \frac{M B T^{1-\alpha}}{1-\alpha} \leq r / 2 ; \\
& \frac{M L T^{1-\alpha}}{1-\alpha} \leq 1 / 2,
\end{aligned}
$$

onde adotamos o seguinte procedimento: primeiramente escolha $r$ e um $T_{0}$ para encontrar $B, L, M$, então escolha um $T$ menor que $T_{0}$ para ter as condições acima satisfeitas.

Com estas escolhas, $G$ leva $B_{r}$ nela mesma e é uma contração na norma de $C\left(\left[t_{0}, t_{0}+T\right], E^{\alpha}\right)$. Então, como $C\left(\left[t_{0}, t_{0}+T\right], E^{\alpha}\right)$ é completo, segue do Teorema do Ponto Fixo de Banach que $G: B_{r} \subset C\left(\left[t_{0}, t_{0}+T\right], E^{\alpha}\right) \rightarrow B_{r}$ tem um único ponto fixo, isto é, existe único $e \in B_{r}$ tal que

$$
e(t)=\mathbf{e}^{-A\left(t-t_{0}\right)} e_{0}+\int_{t_{0}}^{t} \mathbf{e}^{-A(t-s)} f(s, e(s)) d s, \quad t_{0} \leq t \leq t_{0}+T .
$$

Segue então do Teorema 3.4, que $e:\left[t_{0}, t_{0}+T\right] \rightarrow E^{\alpha}$ é uma solução de (3.4).

Para provar a unicidade, suponha que e, ẽ são duas soluções de (3.4), ambas definidas em $\left[t_{0}, t_{2}\right]$. Se elas coincidem em $\left[t_{0}, t_{3}\right]$ mas não coincidem em um intervalo maior, podemos substituir $t_{0}$ por $t_{3}$. Logo, suponha que $e(t) \neq \tilde{e}(t)$ para $t$ arbitrariamente próximo de $t_{0}, t>t_{0}$. Como $e, \tilde{e}$ são contínuas, podemos assumir que isto ocorre para $t \in\left[t_{0}, t_{0}+T\right]$ e $\left\|e(t)-e_{0}\right\|_{E^{\alpha}} \leq r$, $\left\|\tilde{e}(t)-e_{0}\right\|_{E^{\alpha}} \leq r$. Mas as restrições de $e, \tilde{e}$ a $\left[t_{0}, t_{0}+T\right]$ são pontos fixos de $G$, contradizendo a unicidade dos pontos fixos.

Provadas a existência e unicidade estendemos agora as soluções locais ao intervalo maximal de existência. Seja $t_{1}=\sup \left\{T \in \mathbb{R}\right.$ : existe uma solução de (3.4) definida em $\left.\left[t_{0}, t_{0}+T\right)\right\}$. Para cada $t \in\left[t_{0}, t_{1}\right)$ defina $e(t)=\left\{\right.$ o valor em $t$ de uma solução $\tilde{e}:\left[t_{0}, t_{0}+T\right) \rightarrow E^{\alpha}$ de (3.4), com $\left.t_{0}+T>t\right\}$. Como já mostramos $e$ está bem definida e, claramente, é maximal.

Suponha que $t_{1}<\infty$ e que o limite $e_{1}=\lim _{t \rightarrow t_{1}} e(t)$ exista. Se $\left(t_{1}, e_{1}\right) \in U$ então, como já mostrado na primeira parte da prova, existe uma solução fraca $\tilde{e}:\left[t_{1}, t_{1}+T\right] \rightarrow E^{\alpha}$ para algum $T>0, \operatorname{com} \tilde{e}\left(t_{1}\right)=e_{1}$. Então se $\hat{e}:\left[t_{0}, t_{1}+T\right] \rightarrow E^{\alpha}$ é dada por $\hat{e}(t)=e(t), t_{0} \leq t<t_{1} \mathrm{e}$ 
$\hat{e}(t)=\tilde{e}(t), t_{1} \leq t \leq t_{1}+T$, então $\hat{e}$ é uma solução de (3.4), o que contradiz a definição de $t_{1}$. Portanto, se existir o limite $e_{1}=\lim _{t \rightarrow t_{1}} e(t)$ então necessariamente $\left(t_{1}, e_{1}\right) \in \partial U$.

Mostremos que

$$
\frac{\|f(t, e(t))\|_{E}}{1+\|e(t)\|_{E^{\alpha}}} \leq C<\infty, \quad t_{0} \leq t<t_{1},
$$

implica que $\lim _{t \rightarrow t_{1}} e(t)$ existe, o que completará a prova. Do Teorema 2.8, existe $M_{\alpha}>0$ tal que

$$
\left\|\mathbf{e}^{-A(t-s)} f(s, e(s))\right\|_{E^{\alpha}} \leq M_{\alpha}(t-s)^{-\alpha}\|f(s, e(s))\|_{E}, \text { se } t>s .
$$

Daí se $K>0$ é tal que $\left\|\mathbf{e}^{-A\left(t-t_{0}\right)} A^{\alpha} e_{0}\right\|_{E} \leq K\left\|A^{\alpha} e_{0}\right\|_{E}$, usando (3.12) temos

$$
\begin{aligned}
0 \leq\|e(t)\|_{E^{\alpha}} & \leq\left\|A^{\alpha} \mathbf{e}^{-A\left(t-t_{0}\right)} e_{0}\right\|_{E}+\int_{t_{0}}^{t}\left\|A^{\alpha} \mathbf{e}^{-A(t-s)}\right\|_{L(E)}\|f(s, e(s))\|_{E} d s \leq \\
& \leq K\left\|e_{0}\right\|_{E^{\alpha}}+M_{\alpha} C \int_{t_{0}}^{t}(t-s)^{-\alpha} d s+M_{\alpha} C \int_{t_{0}}^{t}(t-s)^{-\alpha}\|e(t)\|_{E^{\alpha}} d s= \\
& =\left(K\left\|e_{0}\right\|_{E^{\alpha}}+M_{\alpha} C{\frac{\left(t_{0}-s\right)^{1-\alpha}}{1-\alpha}}^{t}\right)+M_{\alpha} C \int_{t_{0}}^{t}(t-s)^{-\alpha}\|e(t)\|_{E^{\alpha}} d s= \\
& =a+b \int_{t_{0}}^{t}(t-s)^{\alpha}\|e(s)\|_{E^{\alpha}} d s,
\end{aligned}
$$

$t_{0} \leq t_{1}, a, b \geq 0$. Segue então do Lema 3.2 que existe uma constante $\bar{K}$ dependendo somente de $\alpha$ e $t_{1}$, tal que

$$
\|e(t)\|_{E^{\alpha}} \leq \bar{K} a, t_{0}<t<t_{1},
$$

donde segue que

$$
\|f(t, e(t))\|_{E} \leq C(1+\bar{K} a)=C_{1},
$$

$t_{0}<t<t_{1}$. Provaremos agora que $\|e(s)-e(r)\|_{E^{\alpha}} \rightarrow 0$ quando $s, r \rightarrow t_{1}$. Dado $\epsilon>0$ escolha $0<\epsilon_{1}<t_{1}-t_{0} \operatorname{com} \epsilon_{1}<\frac{\epsilon(1-\alpha)}{4 M_{\alpha} C_{1}}$. Seja $t^{*}=t_{1}-\epsilon_{1}^{1 /(1-\alpha)}$ e seja $0<\delta \leq \epsilon_{1}$ tal que

$$
\left\|\left(\mathbf{e}^{-A\left(s-t^{*}\right)}-\mathbf{e}^{-A\left(r-t^{*}\right)}\right) e\left(t^{*}\right)\right\|_{E^{\alpha}} \leq \frac{\epsilon}{2}
$$

se $|s-r| \leq \delta$. Então para $t^{*} \leq t_{1}-\delta \leq s<t_{1}$,

$$
e(s)=\mathbf{e}^{-A\left(s-t^{*}\right)} e\left(t^{*}\right)+\int_{t^{*}}^{s} \mathbf{e}^{-A(s-\theta)} f(\theta, e(\theta)) d \theta .
$$

Portanto, se $t^{*} \leq t_{1}-\delta \leq s \leq r<t_{1}$

$$
\begin{aligned}
\|e(s)-e(r)\|_{E^{\alpha}} & \leq \frac{\epsilon}{2}+M_{\alpha} C_{1}\left[\int_{t^{*}}^{s}(s-\theta)^{-\alpha} d \theta+\int_{t^{*}}^{r}(r-\theta)^{-\alpha} d \theta\right]= \\
& =\frac{\epsilon}{2}+\frac{M_{\alpha} C_{1}}{1-\alpha}\left[\left(s-t^{*}\right)^{1-\alpha}+\left(r-t^{*}\right)^{1-\alpha}\right] \leq \\
& \leq \frac{\epsilon}{2}+\frac{2 M_{\alpha} C_{1}}{1-\alpha}\left(t_{1}-t^{*}\right)^{1-\alpha}= \\
& =\frac{\epsilon}{2}+\frac{2 M_{\alpha} C_{1}}{1-\alpha} \epsilon_{1}<\epsilon
\end{aligned}
$$

e o resultado segue. 
Exemplo 3.1 Considere o problema de valor inicial

$$
\begin{aligned}
u_{t}=\Delta u+f^{e}(u), \quad \text { em } & \Omega \\
& u(x, 0)=u_{0} \quad \text { se } \quad x \in \Omega,
\end{aligned}
$$

onde $\Omega$ é um domínio limitado em $\mathbb{R}^{n}$ com bordo $C^{2}, \Delta: W^{2, r}(\Omega) \cap W_{0}^{1, r}(\Omega) \rightarrow L^{r}(\Omega)$ e $f^{e}: W_{0}^{1, r}(\Omega) \rightarrow L^{r}(\Omega)$ é dada por $f^{e}(u)(x)=f(u(x))$, onde $f: \mathbb{R} \rightarrow \mathbb{R}$ é de classe $C^{1}$ e $r>n$. Então dado qualquer $u_{0} \in W_{0}^{1, r}$, existe uma única solução $u:[0, T) \rightarrow W_{0}^{1, r}(\Omega)$ de (3.13) definida num intervalo maximal.

Prova. Se $E=L^{r}(\Omega)$ então $E^{1}=W^{2, r}(\Omega) \cap W_{0}^{1, r}(\Omega)$ e $E^{1 / 2}=W_{0}^{1, r}(\Omega)$. Segue do Exemplo $1.1 \mathrm{e}$ da Observação 1.1 que $-\Delta$ é um operador setorial no espaço de Banach $L^{r}(\Omega)$. Mostremos então que $f^{e}: W_{0}^{1, r}(\Omega) \rightarrow L^{r}(\Omega)$ é Lipschitz contínua em limitados e o resultado segue do Teorema 3.5. Como $W_{0}^{1, r} \hookrightarrow C(\bar{\Omega})$, se considerarmos $B_{\delta}(0) \subset W_{0}^{1, r}$, para algum $\delta>0$, temos

$$
M=\sup _{u \in B_{\delta}(0)}\|u\|_{L^{\infty}(\Omega)}<\infty
$$

Além disso, como $f: \mathbb{R} \rightarrow \mathbb{R}$ é de classe $C^{1}$ então $f$ é Lipschitz contínua em limitados, e então exite $L>0$ tal que

$$
|f(s)-f(t)| \leq L|s-t|, \quad \forall s, t \in[-M, M] .
$$

Assim, se $u, v \in B_{\delta}(0)$,

$$
\begin{aligned}
\left\|f^{e}(u)-f^{e}(v)\right\|_{L^{r}(\Omega)} & =\left(\int_{\Omega}\left|f^{e}(u)(x)-f^{e}(v)(x)\right|^{r} d x\right)^{1 / r}= \\
& =\left(\int_{\Omega} \mid f\left((u(x))-f\left(\left.(v(x))\right|^{r} d x\right)^{1 / r} \leq\right.\right. \\
& \leq L\left(\int_{\Omega}|u(x)-v(x)|^{r} d x\right)^{1 / r}= \\
& =L\|u-v\|_{L^{r}(\Omega)} \leq L\|u-v\|_{W_{0}^{1, r}(\Omega)}
\end{aligned}
$$

e o resultado segue.

O teorema a seguir nos dá informações adicionais sobre a regularidade das soluções de (3.4).

Teorema 3.6 Suponha que $E, A, \alpha, U, f$ são como no Teorema 3.5 e admita que $t \mapsto f(t, e)$ satisfaz uma condição de Hölder local com expoente $\theta \leq 1$, próximo de cada $(t, e) \in U$. Então, qualquer solução e $:\left[t_{0}, t_{1}\right] \rightarrow E^{\alpha}$ de (3.4) é tal que $t \mapsto \frac{d}{d t} e(t) \in E^{\gamma}$ é localmente Hölder contínua em $\left(t_{0}, t_{1}\right)$ para qualquer $0 \leq \gamma<\theta$ e $\left\|\frac{d}{d t} e(t)\right\|_{E^{\gamma}}=\mathcal{O}\left(\left(t-t_{0}\right)^{\alpha-\gamma-1}\right)$ quando $t \rightarrow t_{0}^{+}$, se e $(t) e ́$ contínua em $E^{\alpha}$ quando $t \rightarrow t_{0}^{+}$. 
Prova. Como $\left[t_{0}, t_{1}\right]$ é um intervalo compacto, para algum $h_{0}>0$, se $t_{0} \leq t \leq t+h \leq t_{1}$, $0 \leq h \leq h_{0},\|f(t, e(t))\|_{E} \leq B$ e

$$
\|f(t+h, e(t+h))-f(t, e(t))\|_{E} \leq L\left(h^{\theta}+\|e(t+h)-e(t)\|_{E^{\alpha}}\right),
$$

para alguma escolha de $B$ e $L$. Do Teorema 2.8 podemos encontrar $M$ tal que

$$
\begin{aligned}
& \left\|\mathbf{e}^{-A s} z\right\|_{E^{\alpha}} \leq M\|z\|_{E^{\alpha}}, \quad 0 \leq s \leq t_{1}-t_{0} \\
& \left\|\mathbf{e}^{-A s} z\right\|_{E^{\alpha}} \leq M s^{-\alpha}\|z\|_{E}, \quad 0 \leq s \leq t_{1}-t_{0} \\
& \left\|\mathbf{e}^{-A s} z-z\right\|_{E} \leq M \frac{s^{\theta}}{\theta}\|z\|_{E^{\theta}}, \quad 0 \leq s \leq t_{1}-t_{0}
\end{aligned}
$$

e então temos

$$
\begin{aligned}
\|e(t+h)-e(t)\|_{E^{\alpha}} & \leq M^{2} h^{\theta}\left(t-t_{0}\right)^{-\theta}\left\|e\left(t_{0}\right)\right\|_{E^{\alpha}}+\int_{t_{0}}^{t_{0}+h} M(t+h-s)^{-\alpha} B d s+ \\
& +\int_{t_{0}}^{t} M(t-s)^{-\alpha} L\left[h^{\theta}+\|e(s+h)-e(s)\|_{E^{\alpha}}\right] d s \leq \\
& \leq C h^{\theta}\left[\left(t-t_{0}\right)^{-\theta}+\left(t-t_{0}\right)^{-\alpha}\right] \\
& +M L \int_{t_{0}}^{t}(t-s)^{-\alpha}\|e(s+h)-e(s)\|_{E^{\alpha}} d s,
\end{aligned}
$$

$t_{0} \leq t \leq t+h \leq t_{1}, 0 \leq h \leq h_{0}$. Em seguida, usamos o Lema 3.2 para concluir que

$$
\|e(t+h)-e(t)\|_{E^{\alpha}}=\mathcal{O}\left(h^{\theta}\left[\left(t-t_{0}\right)^{-\theta}+\left(t-t_{0}\right)^{-\alpha}\right]\right)
$$

e portanto $g(t):=f(t, e(t))$ satisfaz

$$
\|g(t+h)-g(t)\|_{E} \leq K h^{\theta}\left[\left(t-t_{0}\right)^{-\theta}+\left(t-t_{0}\right)^{-\alpha}\right], \quad\|g(t)\|_{E} \leq B
$$

para $t_{0}<t \leq t+h \leq t_{1}, 0 \leq h \leq h_{0}$.

Mostramos na prova do Teorema 3.4 que

$$
-A \int_{t_{0}}^{t} \mathbf{e}^{-A(t-s)} g(s) d s=-\int_{t_{0}}^{t} A \mathbf{e}^{-A(t-s)}[g(s)-g(t)] d s+\mathbf{e}^{-A\left(t-t_{0}\right)} g(t)-g(t)
$$

e $\log \mathrm{O}$

$$
\frac{d}{d t} e(t)=-A e(t)+g(t)=-A \mathbf{e}^{-A\left(t-t_{0}\right)} e\left(t_{0}\right)+\mathbf{e}^{-A\left(t-t_{0}\right)} g(t)-G(t)
$$

onde $G(t)=\int_{t_{0}}^{t} A \mathbf{e}^{-A(t-s)}[g(s)-g(t)] d s$. Observe que se $0 \leq \gamma<\theta$,

$$
\left\|\mathbf{e}^{-A\left(t-t_{0}\right)} f(t, e(t))\right\|_{E^{\gamma}} \leq M\left(t-t_{0}\right)^{-\gamma} B \text { e }\left\|A \mathbf{e}^{-A\left(t-t_{0}\right)} e_{0}\right\|_{E^{\gamma}} \leq\left\|M\left(t-t_{0}\right)^{-\gamma-1}\right\| e_{0} \|_{E}
$$


Mostraremos agora que $\|G(t+h)-G(t)\|_{E^{\gamma}}=\mathcal{O}\left(h^{\theta-\gamma}\right)$ quando $h \rightarrow 0^{+}$, sempre que $0 \leq \gamma<\theta$ e $t_{0}<t \leq t_{1}$. Veja que

$$
\begin{aligned}
G(t+h)-G(t) & =\int_{t_{0}}^{t_{0}+h} A \mathbf{e}^{-A(t+h-s)}[g(s)-g(t+h)] d s+ \\
& +\int_{t_{0}}^{t} A \mathbf{e}^{-A(t-s)}[g(s+h)-g(t+h)-g(s)+g(t)] d s
\end{aligned}
$$

e que

$$
\|g(t+h)-g(s+h)-g(t)+g(s)\|_{E} \leq 2 K\left[\left(s-t_{0}\right)^{-\theta}+\left(s-t_{0}\right)^{-\alpha}\right] \min \left\{(t-s)^{\theta}, h^{\theta}\right\} .
$$

Logo,

$$
\begin{aligned}
\|G(t+h)-G(t)\|_{E^{\gamma}} & \leq \mathcal{O}(h)+ \\
& +\mathcal{O}\left(\int_{t_{0}}^{t-h} h^{\theta}(t-s)^{-1-\gamma}\left[\left(s-t_{0}\right)^{-\theta}+\left(s-t_{0}\right)^{-\alpha}\right] d s\right)+ \\
& +\mathcal{O}\left(\int_{t-h}^{t}(t-s)^{-1-\gamma+\theta}\left[\left(s-t_{0}\right)^{-\theta}+\left(s-t_{0}\right)^{-\alpha}\right] d s\right)= \\
& =\mathcal{O}\left(h^{\theta-\gamma}\right), \quad \text { quando } h \rightarrow 0^{+}
\end{aligned}
$$




\section{Capítulo 4}

\section{Blow-up}

\subsection{Introdução}

Neste capítulo consideramos o problema de valor inicial e de fronteira

$$
\begin{array}{rll}
u_{t}=\Delta u+f(u) & \text { em } \quad \Omega \times(0, T), \\
u(x, 0)=\phi(x) & \text { se } \quad x \in \Omega, \\
u(x, t)=0 & \text { se } \quad x \in \partial \Omega, 0<t<T,
\end{array}
$$

onde $\Omega$ é um domínio limitado em $\mathbb{R}^{n}$ com bordo $C^{2}$.

Denotamos

$$
\Omega_{T}=\Omega \times(0, T)
$$

e o bordo parabólico de $\Omega_{T}$ por

$$
\Gamma_{T}=\bar{\Omega}_{T} \backslash\{\Omega \times(0, T]\}
$$

Admitimos ao longo deste capítulo que

$$
\begin{array}{r}
f \in C^{1}, \quad f(s)>0 \text { se } s \geq 0, \quad f(s)=0 \text { se } s<0, \\
\phi \in C^{1}(\bar{\Omega}), \quad \phi \geq 0 \quad \text { (não identicamente nula), } \phi=0 \text { sobre } \partial \Omega .
\end{array}
$$

Assim, $\phi \in W_{0}^{1, r}(\Omega)$ para $r>n$ e então segue do Exemplo 3.1 que existe uma única solução de (4.1)-(4.3) definida no intervalo maximal $[0, T)$. Admitimos que $T<\infty$ e então se $U(t)=\max _{x \in \bar{\Omega}} u(x, t)$ temos

$$
U(T-0)=\infty
$$

Há várias condições na literatura que garantem que $T<\infty$ e portanto que ocorra blow-up no sentido de (4.5); o leitor é conduzido a [2], [17], [18], [23] e às referências dadas lá. 
Veja que $u \geq 0\left(\right.$ em $\left.\bar{\Omega}_{T}\right)$. De fato, multiplicando (4.1) por $-u^{-}$e integrando em $\Omega$, para $t \in(0, T)$, temos

$$
-\int_{\Omega} u_{t} u^{-} d x=-\int_{\Omega} \Delta u u^{-} d x-\int_{\Omega} f(u) u^{-} d x .
$$

Observe que para $t \in(0, T)$,

$$
-\int_{\Omega} u_{t} u^{-} d x=\frac{d}{d t}\left[\frac{1}{2} \int_{\Omega}\left(u^{-}\right)^{2} d x\right]
$$

e da Primeira Identidade de Green, temos

$$
\int_{\Omega} \Delta u u^{-} d x+\int_{\Omega} \nabla u^{-} \cdot \nabla u d x=\int_{\partial \Omega} u^{-} \frac{\partial u}{\partial \nu} d s .
$$

Mas segue de (4.3) que $u^{-}=0$ sobre $\partial \Omega$ e então

$$
\int_{\Omega} \Delta u u^{-} d x=-\int_{\Omega} \nabla u^{-} \nabla u d x=\int_{\Omega}\left|\nabla u^{-}\right|^{2} d x .
$$

Daí, como $f(u) u^{-}=f\left(-u^{-}\right) u^{-}=0$, usando (4.7) e (4.8) em (4.6) temos para cada $t \in(0, T)$

$$
\frac{d}{d t}\left[\frac{1}{2} \int_{\Omega}\left(u^{-}\right)^{2} d x\right]=-\int_{\Omega}\left|\nabla u^{-}\right|^{2} d x \leq 0 .
$$

Assim, se $\psi(t)=\frac{1}{2} \int_{\Omega}\left(u^{-}\right)^{2} d x, t \in[0, T]$, temos $\psi^{\prime}(t) \leq 0$ para $t \in(0, T)$ com $\psi(0)=0$ (pois $\phi \geq 0)$ e $\psi$ contínua em $[0, T]$. Logo, $\psi(t) \leq 0$, para $t \in[0, T]$, isto é,

$$
\int_{\Omega}\left(u^{-}\right)^{2} d x \leq 0 \text { para cada } t \in[0, T]
$$

o que implica que $u^{-} \equiv 0$ em $\Omega$ para cada $t \in[0, T]$. Portanto, $u \geq 0$ em $\bar{\Omega}_{T}$.

Assim, como $f(s)>0$ se $s>0$ e $f(0)=0$, segue de (4.1) que

$$
u_{t}-\Delta u \geq 0 \text { em } \Omega_{T}
$$

e então segue do Princípio do Máximo Forte (Teorema B.3) que se o mínimo de $u$ é assumido em $\Omega \times(0, T]$, isto é, se existe $\left(x_{0}, t_{0}\right) \in \Omega \times(0, T]$ tal que

$$
u\left(x_{0}, t_{0}\right)=0
$$

então

$$
u \equiv 0 \text { em } \Omega_{t_{0}}=\Omega \times\left(0, t_{0}\right) .
$$

Mas, por (4.2) temos que $u(x, 0)=\phi(x)$ para $x \in \Omega$ e, por (4.4), $\phi \geq 0$ (não identicamente nula), contradizendo a cotinuidade de $u$ em $\bar{\Omega}_{T}$.

Portanto,

$$
u>0 \text { em } \Omega \times(0, T]
$$


Definição 4.1 Um ponto $x \in \Omega$ é chamado de ponto de blow-up se existe uma sequência $\left(x_{m}, t_{m}\right)$ tal que

$$
t_{m} \uparrow T, \quad x_{m} \rightarrow x \text { e } u\left(x_{m}, t_{m}\right) \rightarrow \infty \text { se } m \rightarrow \infty \text {. }
$$

Estamos interessados no estudo do conjunto de pontos de blow-up e no comportamento de $u$ nos pontos de blow-up. Dividimos nossos resultados de acordo com os dois seguintes casos:

1. o caso simétrico, e

2. o caso não-simétrico.

No caso simétrico, $\Omega$ é uma bola e $\phi, u(., t)$ são funções radiais. Admitimos que $\phi_{r} \leq 0$. Provamos então, para uma classe geral de funções $f$, que o conjunto de blow-up do problema (4.1)-(4.3) consiste de um único ponto $x=0$. Para a função

$$
f(u)=u^{p} \quad, \quad p>1
$$

obtemos também as estimativas

$$
\begin{gathered}
|u(r, t)| \leq \frac{c}{r^{\frac{2}{\gamma-1}}} \quad \text { para qualquer } \gamma<p, \\
\limsup _{t \rightarrow T^{-}}\|u(., t)\|_{L^{q}(\Omega)}<\infty \quad \text { se } q<\frac{n(p-1)}{2}, \\
\liminf _{t \rightarrow T^{-}}\|u(., t)\|_{L^{q}(\Omega)}=\infty \quad \text { se } q>\frac{n(p-1)}{2},
\end{gathered}
$$

e se $\Delta \phi+f(\phi) \geq 0$ e $n=1,2$ ou se $p \leq \frac{n+2}{n-2}$ quando $n \geq 3$, obtemos que

$$
(T-t)^{\beta} u(r, t) \rightarrow \beta^{\beta} \quad \text { quando } t \uparrow T \quad\left(\beta=\frac{1}{p-1}\right)
$$

com a condição de que $r \leq C(T-t)^{\frac{1}{2}}$ para algum $C>0$.

No caso não-simétrico admitimos que $\Omega$ é um domínio convexo e para alguns resultados é pedido que $\Delta \phi+f(\phi) \geq 0$ (e então $u_{t} \geq 0$ pelo Princípio do Máximo). Provamos que o conjunto de blow-up do problema (4.1)-(4.3) é um subconjunto compacto de $\Omega$ e que para $f$ dada por (4.10),

$$
u(x, t) \leq \frac{C}{(T-t)^{\frac{1}{p-1}}} \quad \text { para todo } x \in \Omega
$$


Segue de um resultado de Giga e Kohn [12] que se $n=1,2$ ou se $n \geq 3$ e $p \leq \frac{n+2}{n-2}$, então

$$
\tau^{\beta} u(x+\xi, T-\tau) \rightarrow c_{0} \quad \text { quando } \tau \downarrow 0
$$

com a condição de que $|\xi| \leq C \tau^{\frac{1}{2}}$ para alguma constante C, onde ou $c_{0}=0$ ou $c_{0}=\beta^{\beta}$. Aqui $x$ é um ponto de blow-up qualquer.

Provamos também, no caso não-simétrico, que para $f$ dada por (4.10)

$$
\liminf _{t \rightarrow T^{-}}\|u(., t)\|_{L^{q}(\Omega)}=\infty \quad \text { se } q>\frac{n(p-1)}{2} .
$$

Estimativas de blow-up também foram provadas por Weissler [22], 24] e extensões de (4.11) para o caso $q=\frac{n(p-1)}{2}$ são dadas por Weissler [25] sob algumas restrições sobre $\phi$ e $p$.

A maioria de nossos resultados são afirmados para uma função geral $f$ e então especializado para $f$ como em (4.10). As condições sobre a função geral $f$ são afirmadas em termos de funções auxiliares $F$.

\subsection{O caso simétrico}

Nesta seção consideramos (4.1)-(4.3) no caso em que

$$
\Omega \text { é uma bola } B_{R}=\{|x|<R\}
$$

e admitimos, além de (4.4) que

$$
\phi=\phi(r), \quad \phi^{\prime}(r)<0 \text { se } 0<r \leq R \text { e } \phi^{\prime \prime}(0)<0 .
$$

Lema $4.1 u_{r}<0$ em $\Omega_{T} \cap\{r>0\}$.

Prova. Seja $w=r^{n-1} u_{r}$ em $\bar{\Omega}_{T}$. Considere a seguinte notação

$$
x=\left(x_{1}, \ldots, x_{n}\right), \quad r=|x|=\sqrt{x_{1}^{2}+\ldots+x_{n}^{2}} .
$$

Veja que, para $i=1, \ldots, n$,

$$
\frac{\partial r}{\partial x_{i}}=2 x_{i} \frac{1}{2 \sqrt{x_{1}^{2}+\ldots+x_{n}^{2}}}=\frac{x_{i}}{r} ; \quad \frac{\partial u}{\partial x_{i}}=\frac{\partial u}{\partial r} \frac{\partial r}{\partial x_{i}}=\frac{1}{r} \frac{\partial u}{\partial r} x_{i} .
$$

Logo,

$$
\begin{aligned}
\frac{\partial^{2} u}{\partial x_{i}^{2}} & =\frac{1}{r} \frac{\partial u}{\partial r}+x_{i} \frac{\partial}{\partial x_{i}}\left(\frac{1}{r} \frac{\partial u}{\partial r}\right)= \\
& =\frac{1}{r} \frac{\partial u}{\partial r}+x_{i}\left[\frac{-1}{r^{2}} \frac{\partial r}{\partial x_{i}} \frac{\partial u}{\partial r}+\frac{1}{r} \frac{\partial^{2} u}{\partial x_{i} \partial r}\right]= \\
& =\frac{1}{r} \frac{\partial u}{\partial r}+x_{i}\left[\frac{-1}{r^{3}} x_{i} \frac{\partial u}{\partial r}+\frac{1}{r} \frac{\partial^{2} u}{\partial x_{i} \partial r}\right] .
\end{aligned}
$$


Calculemos $\frac{\partial^{2} u}{\partial x_{i} \partial r}=\frac{\partial}{x_{i}} \frac{\partial u}{\partial r}$. Seja $v=\frac{\partial u}{\partial r}$. Então

$$
\frac{\partial v}{\partial x_{i}}=\frac{\partial v}{\partial r} \frac{\partial r}{\partial x_{i}}=\frac{\partial v}{\partial r} \frac{x_{i}}{r} .
$$

Logo,

$$
\frac{\partial^{2} u}{\partial x_{i} \partial r}=u_{r r} \frac{x_{i}}{r}
$$

Assim, de (4.13) e de (4.14), temos

$$
\frac{\partial^{2} u}{\partial x_{i}^{2}}=\frac{1}{r} \frac{\partial u}{\partial r}-\frac{x_{i}^{2}}{r^{3}} \frac{\partial u}{\partial r}+\frac{1}{r^{2}} u_{r r} x_{i}^{2} .
$$

Logo,

$$
\begin{aligned}
\Delta u & =\sum_{i=1}^{n} \frac{\partial^{2} u}{\partial x_{i}^{2}}= \\
& =\frac{n}{r} \frac{\partial u}{\partial r}-\frac{1}{r} \frac{\partial u}{\partial r}+u_{r r}= \\
& =(n-1) \frac{1}{r} u_{r}+u_{r r} .
\end{aligned}
$$

Mas veja que

$$
w_{r}=(n-1) r^{n-2} u_{r}+r^{n-1} u_{r r}
$$

e então

$$
\frac{1}{r^{n-1}} w_{r}=(n-1) \frac{1}{r} u_{r}+u_{r r}=\Delta u .
$$

Assim, (4.1) transforma-se em

$$
u_{t}-\frac{1}{r^{n-1}} w_{r}=f(u) .
$$

Derivando (4.16) com respeito a $r$ obtemos

$$
w_{t}+\frac{n-1}{r} w_{r}-w_{r r}-f^{\prime}(u) w=0 \mathrm{em} \Omega_{T},
$$

onde $f^{\prime}$ é função limitada. De fato, como $u$ é contínua em $\bar{\Omega} \times[0, T-\delta]$, subconjunto compacto de $\bar{\Omega}_{T}$, para cada $\delta>0$, temos que $u(\bar{\Omega} \times[0, T-\delta])$ é limitado em $\mathbb{R}$. Daí, como $f^{\prime}$ é contínua (por (4.4)), temos $f^{\prime}$ função limitada.

Além disso, como $u=0$ sobre $\partial \Omega$ e $u>0$ em $\Omega_{T}$ (por 4.9) temos, para $t \in[0, T$ ),

$$
u_{r}(R, t)=\lim _{\delta \rightarrow 0^{-}} \frac{u(R+\delta, t)-u(R, t)}{\delta}=\lim _{\delta \rightarrow 0^{-}} \frac{u(R+\delta, t)}{\delta} \leq 0,
$$

donde segue que $w=r^{n-1} u_{r} \leq 0$ sobre $\partial \Omega \times[0, T)$. Além disso, segue de (4.12) que $w \leq 0$ sobre $t=0$ e então

$$
\max _{\Gamma_{T}} w=0 .
$$


Agora veja que $\bar{w}=\max _{\Gamma_{T}} w-w$ satisfaz (4.17) com $f^{\prime}$ limitada e $\bar{w} \geq 0$ em $\Gamma_{T}$. Então segue do Princípio do Mínimo (Teorema B.5) que

$$
\bar{w} \geq 0 \text { em } \Omega \times(0, T]
$$

isto é, $w \leq 0$ em $\Omega \times(0, T]$. Assim, segue do Princípio do Mínimo Forte (Teorema B.6) que se existe $\left(x_{0}, t_{0}\right) \in \Omega_{T} \cap\{r>0\}$ tal que $\bar{w}\left(x_{0}, t_{0}\right)=0$, então

$$
\bar{w}=0 \mathrm{em} \Omega_{t_{0}} \cap\{r>0\},
$$

isto é,

$$
w=\max _{\Gamma_{T}}=0 \text { em } \Omega_{t_{0}} \cap\{r>0\} .
$$

Mas, usando novamente (4.12), temos que $w<0$ em $(\Omega \cap\{r>0\}) \times\{t=0\}$, contradizendo a continuidade de $w$. Portanto,

$$
w<0 \text { em } \Omega_{T} \cap\{r>0\}
$$

o que termina a prova.

Queremos obter a seguir uma estimativa por baixo de $\left|w_{r}\right|$ próximo de $r=0$. Introduzimos uma função

$$
J=w+c(r) F(u)
$$

onde $c, F$ são funções positivas a serem determinadas tais que

$$
c \in C^{1}(\Omega), F \in C^{1}\left(\Omega_{T}\right), F(0)=0, F^{\prime} \geq 0 \text { e } F^{\prime \prime} \geq 0
$$

Nosso objetivo é mostrar que $J \leq 0$ em $\Omega_{T}$. Calculamos, usando (4.15) e (4.17)

$$
\begin{aligned}
J_{t}+\frac{n-1}{r} J_{r}-J_{r r} & =f^{\prime}(u) w+c F^{\prime} f+\frac{2(n-1)}{r} c F^{\prime} u_{r}+\frac{n-1}{r} c^{\prime} F-c F^{\prime \prime} u_{r}^{2}-2 c^{\prime} F^{\prime} u_{r}-c^{\prime \prime} F \equiv \\
& \equiv B .
\end{aligned}
$$

Usando as relações $u_{r}=\frac{w}{r^{n-1}}$ e $w=-c F+J$ encontramos

$B=b J-\frac{2 F^{\prime \prime}}{r^{2 n-2}} J^{2}-c\left(f^{\prime} F-f F^{\prime}\right)-\frac{c^{3}}{r^{2 n-2}} F^{\prime \prime} F^{2}+\frac{2 c c^{\prime}}{r^{n-1}} F^{\prime} F+\frac{n-1}{r} c^{\prime} F-\frac{2(n-1)}{r^{n}} F^{\prime} F-c^{\prime \prime} F$,

onde

$$
b=f^{\prime}+\frac{2(n-1)}{r^{n}} c F^{\prime}+\frac{2 c^{2} F^{\prime \prime} F}{r^{2 n-2}}-\frac{2 c^{\prime} F^{\prime}}{r^{n-1}} .
$$


Segue então de (4.20) que

$$
J_{t}+\frac{n-1}{r} J_{r}-J_{r r}-b J \leq 0 \quad(0<r<R, \quad 0<t \leq T)
$$

com a condição de que

$$
f^{\prime} F-f F^{\prime}-\frac{2 c^{\prime}}{r^{n-1}} F^{\prime} F+\frac{2(n-1)}{r^{n}} c F^{\prime} F+\left(c^{\prime \prime}-\frac{n-1}{r} c^{\prime}\right) \frac{F}{c} \geq 0,
$$

onde usamos o fato de que $c$ é função positiva e $F^{\prime \prime} \geq 0$.

Agora veja que, claramente, $J$ não pode assumir máximo sobre $\{r=R\} \times[0, T]$ se $J_{r} \leq 0$ sobre $\{r=R\} \times[0, T]$. Encontremos então condições para que tenhamos $J_{r} \leq 0$ sobre $\{r=R\} \times[0, T]$. Veja que, para $0 \leq t \leq T$,

$$
J_{r}(R, t)=w_{r}(R, t)+c(R) F^{\prime}(u(R, t)) u_{r}(R, t)+c^{\prime}(R) F(u(R, t)) .
$$

Segue do Lema 4.1, que $u_{r} \leq 0$ sobre $\Omega_{T} \cap\{r>0\}$ e da continuidade de $u_{r}$ temos $u_{r} \leq 0$ sobre $\partial \Omega \times[0, T]$. Assim,

$$
J_{r}(R, t) \leq w_{r}(R, t)+c^{\prime}(R) F(u(R, t))
$$

e usando (4.16) temos,

$$
\begin{aligned}
J_{r}(R, t) & \leq-R^{n-1}\left[f(u(R, t))-u_{t}(R, t)\right]+c^{\prime}(R) F(u(R, t))= \\
& =-R^{n-1} f(0)+c^{\prime}(R) F(0),
\end{aligned}
$$

para $0 \leq t \leq T$, onde usamos o fato de que como $u=0$ sobre $\partial \Omega((4.3))$, temos

$$
u_{t}(R, t)=\lim _{h \rightarrow 0} \frac{u(R, t+h)-u(R, t)}{h}=0 .
$$

Portanto, $J_{r} \leq 0$ sobre $\{r=R\} \times[0, T]$ se

$$
F(0) c^{\prime}(R) \leq R^{n-1} f(0)
$$

Além disso, veja que

$$
J(r, 0)<0, \quad 0<r<R \quad \Leftrightarrow \quad \frac{c(r)}{r^{n}}<\frac{-\phi^{\prime}(r)}{r F(\phi(r))}, \quad 0<r<R .
$$

Mas,

$$
\min _{0<r<R} \frac{-\phi^{\prime}(r)}{r F(\phi(r))}=\frac{-\phi^{\prime}\left(r_{0}\right)}{r_{0} F\left(\phi\left(r_{0}\right)\right)},
$$

para algum $r_{0} \in(0, R)$. Logo,

$$
\min _{0<r<R} \frac{-\phi^{\prime}(r)}{r F(\phi(r))} \leq 0 \quad \Leftrightarrow \quad \phi^{\prime}\left(r_{0}\right) \geq 0,
$$


o que é impossível segundo (4.12). Portanto,

$$
\min _{0<r<R} \frac{-\phi^{\prime}(r)}{r F(\phi(r))}=C>0 .
$$

Assim, voltando em (4.24), como $J(0,0)=0$, temos que $J(r, 0) \leq 0$, para $0 \leq r<R$ se

$$
\sup _{0<r<R} \frac{c(r)}{r^{n}} \quad \text { for suficientemente pequeno. }
$$

Assim, se (4.22), (4.23) e (4.25) são satisfeitas temos que $J$ satisfaz

$$
J_{t}+\frac{n-1}{r} J_{r}-J_{r r}-b J \leq 0 \text { em } \Omega \backslash\{0\} \times(0, T]
$$

e $J \leq 0$ sobre $\Gamma_{T}$. Então se

$$
b \text { é limitado para } 0<r<R,
$$

segue do Princípio do Mínimo (Teorema B.5) que $J \leq 0$ em $\Omega_{T} \cap\{r>0\}$. Daí se escolhermos

$$
c(0)=0
$$

como

$$
J=w+c(r) F(u)=r^{n-1} u_{r}+c(r) F(u),
$$

temos que $J=0$ sobre $\{r=0\}$ e o resultado segue.

Veja então que escolhendo $c=\varepsilon r^{n} \operatorname{com} \varepsilon$ suficientemente pequeno, todas as condições (4.23), $(4.25)$ e (4.27) são satisfeitas e (4.22) torna-se

$$
f^{\prime} F-f F^{\prime} \geq 2 \varepsilon F F^{\prime}
$$

O lema a seguir resume o que acabamos de comentar:

Lema 4.2 Se $c=\varepsilon r^{n}$ com $\varepsilon$ suficientemente pequeno e $F$ satisfaz (4.28) então

$$
-r^{n-1} u_{r} \geq c(r) F(u)
$$

Prova. Como comentado anteriormente, se $c=\varepsilon r^{n} \operatorname{com} \varepsilon$ suficientemente pequeno e se $F$ satisfaz (4.28) então todas as condições (4.23), (4.25) e (4.27) estão satisfeitas, o que é suficiente para garantir que $J \leq 0$ em $\Omega_{T}$, implicando (4.29).

Admita a seguir que

$$
\int^{\infty} \frac{d \tau}{F(\tau)}<\infty
$$


isto é,

$$
\int_{\alpha}^{\infty} \frac{d \tau}{F(\tau)}<\infty, \quad \forall \alpha \in \mathbb{R}
$$

Seja

$$
G(s)=\int_{s}^{\infty} \frac{d \tau}{F(\tau)}
$$

Mostremos que

$$
G^{\prime}(s)=\frac{-1}{F(s)} .
$$

De fato, suponhamos sem perda de generalidade $0<h<1$. Veja que

$$
\frac{1}{F(s)}=\frac{1}{h} \int_{s}^{s+h} \frac{1}{F(s)} d \tau
$$

e então

$$
\begin{aligned}
\left|\frac{G(s+h)-G(s)}{h}+\frac{1}{F(s)}\right| & =\left|\frac{1}{h}\left(\int_{s+h}^{\infty} \frac{1}{F(\tau)} d \tau-\int_{s}^{\infty} \frac{1}{F(\tau)} d \tau\right)+\frac{1}{h} \int_{s}^{s+h} \frac{1}{F(s)} d \tau\right|= \\
& =\left|-\frac{1}{h} \int_{s}^{s+h} \frac{1}{F(\tau)} d \tau+\frac{1}{h} \int_{s}^{s+h} \frac{1}{F(s)} d \tau\right|= \\
& =\left|\frac{1}{h} \int_{s}^{s+h} \frac{F(\tau)-F(s)}{F(s) F(\tau)} d \tau\right| \leq \\
& \leq \frac{1}{h} \frac{1}{\min _{x \in[s, s+1]}(F(x))^{2}} \max _{\tau \in[s, s+h]}|F(\tau)-F(s)| h= \\
& =\frac{1}{(F(s))^{2}} \max _{\tau \in[s, s+h]}|F(\tau)-F(s)| \rightarrow 0 \quad \text { quando } h \rightarrow 0,
\end{aligned}
$$

uma vez que $F^{\prime} \geq 0$ e $F(\tau) \rightarrow F(s)$ quando $h \rightarrow 0$.

Assim temos

$$
(G(u))_{r}=G^{\prime}(u) u_{r}=-\frac{1}{F(u)} u_{r}
$$

e (4.29) nos dá

$$
(G(u))_{r} \geq \frac{c(r)}{r^{n-1}}
$$

Daí, por integração,

$$
G(u(r, t))-G(u(0, t)) \geq \int_{0}^{r} \frac{c(r)}{r^{n-1}} d r
$$

e como $G(u(0, t)) \geq 0$ temos

$$
G(u(r, t)) \geq \int_{0}^{r} \frac{c(r)}{r^{n-1}} d r .
$$


No caso $c(r)=\varepsilon r^{n}$, temos

$$
G(u(r, t)) \geq \frac{1}{2} \varepsilon r^{2} .
$$

Teorema 4.1 Admitindo (4.22), (4.23), (4.25), (4.27) e (4.30), o ponto $r=0$ é o único ponto de blow-up para (4.1)-(4.3).

Prova. Mostremos inicialmente que se $r>0$ então $r$ não é ponto de blow-up de (4.1)-(4.3). Para isso suponhamos que para algum $r>0, u(r, t) \rightarrow \infty$ quando $t \rightarrow T$. Mostremos então que $G(u(r, t)) \rightarrow 0$ quando $t \rightarrow T$, o que contraria (4.33).

Fixe $\alpha \in \mathbb{R}$ qualquer. Como $u(r, t) \rightarrow \infty$ quando $t \rightarrow T$, exite $t$ suficientemente grande $(t \sim T)$ tal que $u(r, t)>\alpha$. Então, como admitimos (4.30), podemos escrever

$$
\int_{\alpha}^{\infty} \frac{d \tau}{F(\tau)}=\int_{\alpha}^{u(r, t)} \frac{d \tau}{F(\tau)}+\int_{u(r, t)}^{\infty} \frac{d \tau}{F(\tau)}
$$

isto é,

$$
G(\alpha)=\int_{\alpha}^{u(r, t)} \frac{d \tau}{F(\tau)}+G(u)
$$

Assim, como

$$
\int_{\alpha}^{u(r, t)} \frac{d \tau}{F(\tau)} \leq \int_{\alpha}^{\infty} \frac{d \tau}{F(\tau)}<\infty
$$

temos

$$
G(u)=G(\alpha)-\int_{\alpha}^{u(r, t)} \frac{d \tau}{F(\tau)} .
$$

Mas, como $u(r, t) \rightarrow \infty$ quando $t \rightarrow T$, segue de (4.31) que

$$
\int_{\alpha}^{u(r, t)} \frac{d \tau}{F(\tau)} \rightarrow G(\alpha) \quad \text { quando } \quad t \rightarrow T
$$

Segue então de (4.35) que

$$
G(u) \rightarrow 0 \quad \text { quando } \quad t \rightarrow T
$$

o que contradiz (4.34).

Portanto, segue de (4.5) que $r=0$ é o único ponto de blow-up para (4.1)-(4.3).

Considere a equação

$$
u_{t}=\Delta u+u^{p} \quad(p>1) .
$$

Neste caso tomamos $F(s)=s^{\gamma}, 1<\gamma<p$ e $c(r)=\varepsilon r^{n+\delta}$, para $\delta>0$ qualquer. Então (4.28) se reduz a

$$
(p-\gamma) u^{p+\gamma-1} \geq 2 \varepsilon \gamma u^{2 \gamma-1}
$$

que é válido se $\varepsilon$ é suficientemente pequeno. A correspondente função $G$ é

$$
G(s)=\frac{1}{\gamma-1} \frac{1}{s^{\gamma-1}}
$$


onde usamos o fato de que $\lim _{\tau \rightarrow \infty} \frac{1}{1-\gamma} \frac{1}{\tau^{\gamma-1}}=0$, uma vez que $\gamma>1$. Então segue de (4.33) que para $1<\gamma<0$,

$$
\begin{aligned}
{\frac{(u(r, t))^{1-\gamma}}{\gamma-1}}^{1-\gamma} & \geq \int_{0}^{r} \varepsilon \frac{r^{n+\delta}}{r^{n-1}} d r= \\
& =\varepsilon \int_{0}^{r} r^{\delta+1} d r= \\
& =\varepsilon \frac{r^{\delta+2}}{\delta+2}
\end{aligned}
$$

isto é,

$$
(u(r, t))^{\gamma-1} \leq \frac{\delta+2}{\gamma-1} \frac{1}{\varepsilon} \frac{1}{r^{\delta+2}}
$$

donde segue que

$$
u(r, t) \leq\left(\frac{\delta+2}{\varepsilon(\gamma-1)}\right)^{\frac{1}{\gamma-1}} \frac{1}{r^{\frac{2}{\gamma-1}}} .
$$

Portanto, existe $C>0$ tal que

$$
u(r, t) \leq \frac{C}{r^{\frac{2}{\gamma-1}}}, \quad \text { para } \quad \text { qualquer } 1<\gamma<p .
$$

Asssim:

Teorema 4.2 Para (4.36), (4.2), (4.3), o ponto $r=0$ é o único ponto de blow-up e a estimativa (4.37) vale. Consequentemente

$$
\limsup _{t \rightarrow T^{-}}\|u(\cdot, t)\|_{L^{q}(\Omega)}<\infty \quad \text { se } q<\frac{n(p-1)}{2} .
$$

Prova. Se $q<\frac{n(p-1)}{2}$ então temos $p>\frac{2 q+n}{n}$. Fixe $\frac{2 q+n}{n}<\gamma<p$ e agora estime $\|u(\cdot, t)\|_{L^{q}(\Omega)}$ para cada $t$ fixado:

$$
\begin{aligned}
\|u(\cdot, t)\|_{L^{q}(\Omega)}^{q} & =\int_{\Omega}|u(x, t)|^{q} d x= \\
& =\int_{0}^{R}\left[\int_{\partial B_{R}(0)}|u(x, t)|^{q} d S\right] d r .
\end{aligned}
$$

Usando (4.37),

$$
\begin{aligned}
\|u(\cdot, t)\|_{L^{q}(\Omega)}^{q} & \leq C \int_{0}^{R} \frac{1}{\frac{2 q}{r^{\frac{2 q}{\gamma-1}}}} \int_{\partial B_{R}(0)} d S d r= \\
& =C \int_{0}^{R} \frac{1}{r^{\frac{2 q}{\gamma-1}}} n \alpha(n) r^{n-1} d r= \\
& =C \lim _{\varepsilon \rightarrow 0}\left[\int_{\varepsilon}^{R} n \alpha(n) r^{n-1-\frac{2 q}{(\alpha-1)}} d r\right]= \\
& =C n \alpha(n) \lim _{\varepsilon \rightarrow 0}\left[\left.\frac{r^{n-\frac{2 q}{(\gamma-1)}}}{n-\frac{2 q}{(\gamma-1)}}\right|_{\varepsilon} ^{R}\right],
\end{aligned}
$$


onde $\alpha(n)$ denota a área da superfície esférica (n-1)-dimensional. Mas veja que como $\gamma>\frac{2 q+n}{n}$, então $n-\frac{2 q}{(\gamma-1)}>0$ e então

$$
\lim _{\varepsilon \rightarrow 0}\left[\frac{\varepsilon^{n-\frac{2 q}{(\gamma-1)}}}{n-\frac{2 q}{(\gamma-1)}}\right]=0 .
$$

Logo,

$$
\|u(\cdot, t)\|_{L^{q}(\Omega)} \leq\left\{C n \alpha(n) \frac{R^{n-\frac{2 q}{(\gamma-1)}}}{n-\frac{2 q}{(\gamma-1)}}\right\}^{1 / q}
$$

e então, como o lado direito da desigualdade acima independe de $t$, temos (4.38).

Note que para $q>\frac{n(p-1)}{2}$ não há escolha em $\gamma \in(1, p)$ que torne a integral

$$
\int_{\Omega}|u(x, t)|^{q} d x
$$

convergente, pois

$$
n-\frac{2 q}{\gamma-1}<n-\frac{2}{\gamma-1} \frac{n(p-1)}{2}
$$

e

$$
n-\frac{n(p-1)}{\gamma-1}=n\left(1-\frac{p-1}{\gamma-1}\right)=\frac{n}{\gamma-1}(\gamma-p)<0 .
$$

Observação $4.1 \mathrm{Na}$ seção 4.3 provamos um complemento de (4.38) (para qualquer domínio convexo $\Omega$ ):

$$
\liminf _{t \rightarrow T^{-}}\|u(\cdot, t)\|_{L^{q}(\Omega)}=\infty \quad \text { se } q>\frac{n(p-1)}{2} .
$$

Segue do Lema 4.1 que para cada $t \in(0, T),(0, t)$ é ponto de máximo de $u$ em $\Omega \times\{t\}$ e então $\Delta u(0, t) \leq 0$ para cada $t \in(0, T)$. Assim, note que a função $U(t)=u(0, t)$ satisfaz

$$
U_{t}=u_{t}(0, t)=\Delta u(0, t)+f(u(0, t)) \leq f(U) .
$$

A seguir admita que

$$
\int^{\infty} \frac{d \tau}{f(\tau)}<\infty
$$

e denote

$$
\hat{f}(s)=\int_{s}^{\infty} \frac{d \tau}{f(\tau)} .
$$

Agindo de maneira análoga à demonstração de (4.32) temos que

$$
(\hat{f}(U))_{t}=\hat{f}^{\prime}(U) U_{t}=-\frac{1}{f(U)} U_{t}
$$


e usando (4.39) obtemos que

$$
-(\hat{f}(U))_{t} \leq 1
$$

e por integração em $t$ temos

$$
\hat{f}(U(t))-\hat{f}(U(T)) \leq T-t .
$$

Mas, como no caso especial (4.36) $x=0$ é ponto de blow-up de (4.1)-(4.3) (Teorema 4.1), temos $\hat{f}(U(T))=0$ e então segue de (4.43) que

$$
\hat{f}(U(t)) \leq T-t .
$$

Assim, no caso especial (4.36) obtemos que

$$
u(0, t) \geq \frac{c}{(T-t)^{1 /(p-1)}} \quad(c>0) .
$$

De fato, em (4.36) temos $f(\tau)=\tau^{p}, p>1$ e então

$$
\begin{aligned}
\hat{f}(U(t)) & =\hat{f}(U(0, t))= \\
& =\int_{u(0, t)}^{\infty} \frac{d \tau}{\tau^{p}}= \\
& =\lim _{\tau \rightarrow \infty}\left[\frac{1}{(1-p) \tau^{(p-1)}}-\frac{1}{(1-p)(u(0, t))^{(p-1)}}\right]= \\
& =\frac{1}{(p-1)(u(0, t))^{(p-1)}},
\end{aligned}
$$

uma vez que $(p-1)>0$. Usando (4.44) temos

$$
\frac{1}{(p-1)(u(0, t))^{(p-1)}} \leq T-t
$$

e então

$$
u(0, t) \geq \frac{1}{(p-1)^{\frac{1}{p-1}}(T-t)^{\frac{1}{p-1}}} .
$$

Como $p>1$, temos

$$
u(0, t) \geq \frac{c}{(T-t)^{\frac{1}{p-1}}}, \quad c>0,
$$

como queríamos.

Na seção 4.4 provamos para o caso geral não-simétrico que (com a condição de que $\Delta \phi+f(\phi) \geq 0)$

$$
u_{t} \geq \varepsilon f(u),
$$

para algum $\varepsilon>0$ pequeno. Isto segue para uma grande classe de funções $f$, que inclui a função em (4.36). Agora veja que, como comentado anteriormente,

$$
(\hat{f}(U))_{t}=-\frac{1}{f(U)} \cdot U_{t}
$$


e então de (4.47) temos

$$
-(\hat{f}(U))_{t} \geq \varepsilon
$$

Usando novamente o fato de que $\hat{f}(U(T))=0$, obtemos por integração que

$$
\hat{f}(U(t)) \geq \varepsilon(T-t)
$$

que é um complemento a (4.44). Em particular, para o caso (4.36), temos

$$
u(0, t) \leq \frac{C}{(T-t)^{\frac{1}{(p-1)}}} \quad(C>0),
$$

onde usamos (4.46) e (4.48)

Observação 4.2 Segue de um resultado de Giga e Kohn [12] para soluções de (4.36), (4.2), (4.3), que se (4.45) e (4.49) são satisfeitas e se $n=1,2$ ou $n \geq 3$ e $p \leq \frac{(n+2)}{(n-2)}$, então

$$
(T-t)^{\beta} u(r, t) \longrightarrow \beta^{\beta} \quad \text { quando } \quad t \uparrow T \quad\left(\beta=\frac{1}{p-1}\right)
$$

desde que $r \leq C(T-t)^{\frac{1}{2}}$ para alguma constante $C$.

\subsection{O caso não-simétrico}

Nesta seção e na seção 4.4 estudamos o caso não-simétrico em dimensão qualquer e admitimos, em adição a (4.4), que

$$
\Omega \text { é um domínio convexo em } \mathbb{R}^{n} \text {. }
$$

Denotamos

$$
\tilde{f}(s)=\int_{0}^{s} f(\tau) d \tau
$$

Teorema 4.3 Seja $0<t_{0}<T, u_{0}=U\left(t_{0}\right)=\max _{x \in \bar{\Omega}} u\left(x, t_{0}\right)$. Se

$$
\tilde{f}\left(u_{0}\right) \geq \tilde{f}(\phi)+\frac{1}{2}|\nabla \phi|^{2} \quad \text { em } \Omega
$$

então

$$
\frac{1}{2}|\nabla u|^{2} \leq \tilde{f}\left(u_{0}\right)-\tilde{f}(u) \text { em } \Omega_{t_{0}} .
$$

Prova. Considere a função $w=u_{0}-u$. Então

$$
w_{t}=-u_{t} \text { e } \Delta w=-\Delta u
$$


Logo,

$$
w_{t}-\Delta w=-u_{t}+\Delta u .
$$

Além disso,

$$
f\left(u_{0}-w\right)=f\left(u_{0}-u_{0}+u\right)=f(u)
$$

e então segue de (4.1) que

$$
w_{t}-\Delta w=-h(w) \text { em } \Omega_{t_{0}},
$$

para $h(w)=f\left(u_{0}-w\right)$. Agora, segue de (4.2) e (4.3) que

$$
w(x, 0)=u_{0}-u(x, 0)=u_{0}-\phi(x) \text { em } \Omega
$$

e

$$
w(x, t)=u_{0}-u(x, t)=u_{0} \text { sobre } \partial \Omega \times(0, T) .
$$

Portanto, $w=u_{0}-u$ satisfaz

$$
\begin{aligned}
& w_{t}-\Delta w=-h(w) \text { em } \Omega_{t_{0}} \\
& w(x, 0)=u_{0}-\phi(x) \text { em } \Omega \\
& w(x, t)=u_{0} \text { sobre } \partial \Omega \times(0, T),
\end{aligned}
$$

onde $h(w)=f\left(u_{0}-w\right)$. Denote

$$
H(w)=\int_{0}^{w} h(s) d s
$$

e introduza a função

$$
J=\frac{1}{2}|\nabla w|^{2}-H(w) .
$$

Veja que se $\nabla w=\left(\frac{\partial w}{\partial x_{1}}, \ldots, \frac{\partial w}{\partial x_{n}}\right)$ então

$$
\frac{1}{2}|\nabla w|^{2}=\frac{1}{2} \sum_{j=1}^{n}\left(\frac{\partial w}{\partial x_{j}}\right)^{2} .
$$

Logo,

$$
\left(\frac{1}{2}|\nabla w|^{2}\right)_{t}=\sum_{j=1}^{n} \frac{\partial w}{\partial x_{j}} \frac{\partial w}{\partial t \partial x_{j}}
$$

$$
\frac{\partial}{\partial x_{i}}\left(\frac{1}{2}|\nabla w|^{2}\right)=\sum_{j=1}^{n} \frac{\partial w}{\partial x_{j}} \frac{\partial^{2} w}{\partial x_{i} \partial x_{j}}, \quad \text { para } i=1,2, \ldots, n,
$$

donde segue que

$$
\frac{\partial^{2}}{\partial x_{i}^{2}}\left(\frac{1}{2}|\nabla w|^{2}\right)=\sum_{j=1}^{n}\left[\frac{\partial^{2} w}{\partial x_{i} \partial x_{j}} \frac{\partial^{2} w}{\partial x_{i} \partial x_{j}}+\frac{\partial w}{\partial x_{j}} \frac{\partial^{3} w}{\partial^{2} x_{i} \partial x_{j}}\right]
$$


para $i=1, \ldots, n$ e então

$$
\begin{aligned}
\Delta\left(\frac{1}{2}|\nabla w|^{2}\right) & =\sum_{i=1}^{n} \frac{\partial^{2}}{\partial x_{i}^{2}}\left(\frac{1}{2}|\nabla w|^{2}\right)= \\
& =\sum_{i=1}^{n} \sum_{j=1}^{n}\left[\left(\frac{\partial^{2} w}{\partial x_{i} \partial x_{j}}\right)^{2}+\frac{\partial w}{\partial x_{j}} \frac{\partial^{3} w}{\partial^{2} x_{i} \partial x_{j}}\right]
\end{aligned}
$$

Por outro lado, usando o Teorema Fundamental do Cálculo, temos

$$
(H(w))_{t}=h(w) w_{t}
$$

$\mathrm{e}$

$$
\frac{\partial}{\partial x_{i}} H(w)=h(w) w_{x_{i}}, \quad \text { para } i=1,2, \ldots, n,
$$

donde segue que

$$
\frac{\partial^{2}}{\partial x_{i}^{2}} H(w)=h^{\prime}(w)\left(\frac{\partial w}{\partial x_{i}}\right)^{2}+h(w) \frac{\partial^{2} w}{\partial x_{i}^{2}}, \quad \text { para } i=1,2, \ldots, n
$$

e então

$$
\begin{aligned}
\Delta H(w) & =\sum_{i=1}^{n} \frac{\partial^{2}}{\partial x_{i}^{2}} H(w)= \\
& =h^{\prime}(w)|\nabla w|^{2}+h(w) \Delta w .
\end{aligned}
$$

Aplicando (4.57), (4.59), (4.60) e (4.62), a (4.56) obtemos

$$
\begin{aligned}
J_{t}-\Delta J= & \sum_{j=1}^{n} \frac{\partial w}{\partial x_{j}} \cdot \frac{\partial w}{\partial t \partial x_{j}}-h(w) w_{t}-\sum_{i=1}^{n} \sum_{j=1}^{n}\left(\frac{\partial^{2} w}{\partial x_{i} \partial x_{j}}\right)^{2}-\sum_{i=1}^{n} \sum_{j=1}^{n} \frac{\partial w}{\partial x_{j}} \frac{\partial^{3} w}{\partial^{2} x_{i} \partial x_{j}}+ \\
& +h^{\prime}(w)|\nabla w|^{2}+h(w) \Delta w=h(w)\left[\Delta w-w_{t}\right]-\sum_{i, j}\left(\frac{\partial^{2} w}{\partial x_{i} \partial x_{j}}\right)^{2}+h^{\prime}(w)|\nabla w|^{2}+ \\
& +\left[\sum_{j} \frac{\partial w}{\partial x_{j}} \frac{\partial w}{\partial t \partial x_{j}}-\sum_{i, j} \frac{\partial w}{\partial x_{j}} \frac{\partial^{3} w}{\partial x_{i}^{2} \partial x_{j}}\right] .
\end{aligned}
$$

Agora veja que como $h(w)=\Delta w-w_{t}($ por $(4.55))$ temos

$$
\frac{\partial h(w)}{\partial x_{j}}=\frac{\partial}{\partial x_{j}}\left[\Delta w-w_{t}\right]=\sum_{i=1}^{n} \frac{\partial^{3} w}{\partial x_{i}^{2} \partial x_{j}}-\frac{\partial w}{\partial t \partial x_{j}},
$$

para $j=1, \ldots, n$. Mas como

$$
\frac{\partial h(w)}{\partial x_{j}}=h^{\prime}(w) \frac{\partial w}{\partial x_{j}}, \text { para } j=1, \ldots, n
$$


usando (4.64) temos

$$
h^{\prime}(w)\left(\frac{\partial w}{\partial x_{j}}\right)^{2}=\frac{\partial h(w)}{\partial x_{j}} \frac{\partial w}{\partial x_{j}}=\sum_{i} \frac{\partial w}{\partial x_{j}} \frac{\partial^{3} w}{\partial x_{j} \partial x_{j}^{2}}-\frac{\partial w}{\partial t \partial x_{j}} \frac{\partial w}{\partial x_{j}},
$$

para $j=1, \ldots, n$. Logo,

$$
h^{\prime}(w)|\nabla w|^{2}=h^{\prime}(w) \sum_{j=1}^{n}\left(\frac{\partial w}{\partial x_{j}}\right)^{2}=\sum_{i, j} \frac{\partial w}{\partial x_{j}} \frac{\partial^{3} w}{\partial x_{j} \partial x_{i}^{2}}-\sum_{j} \frac{\partial w}{\partial x_{j}} \frac{\partial w}{\partial t \partial x_{j}} .
$$

Voltando em (4.63), temos

$$
J_{t}-\Delta J=h^{2}(w)-\sum_{i, j}\left(\frac{\partial^{2} w}{\partial x_{i} \partial x_{j}}\right)^{2}
$$

Além disso, de (4.58) e (4.61) temos que, para $i=1, \ldots, n$,

$$
J_{x_{i}}=\sum_{j=1}^{n} \frac{\partial w}{\partial x_{j}} \frac{\partial^{2} w}{\partial x_{i} \partial x_{j}}-h(w) w_{x_{i}}
$$

e então

$$
J_{x_{i}}+h(w) w_{x_{i}}=\sum_{j=1}^{n} \frac{\partial w}{\partial x_{j}} \frac{\partial^{2} w}{\partial x_{i} \partial x_{j}}=\nabla w \cdot \nabla w_{x_{i}} \leq|\nabla w|\left|\nabla w_{x_{i}}\right| .
$$

Logo,

$$
\sum_{i}\left(J_{x_{i}}+h(w) w_{x_{i}}\right)^{2} \leq \sum_{i}|\nabla w|^{2}\left|\nabla w_{x_{i}}\right|^{2}=|\nabla w|^{2} \sum_{i, j}\left(\frac{\partial^{2} w}{\partial x_{i} x_{j}}\right)^{2} .
$$

Daí, aplicando (4.66) a (4.65) temos

$$
\begin{aligned}
J_{t}-\Delta J & \leq h^{2}(w)-\frac{1}{|\nabla w|^{2}} \sum_{i}\left(J_{x_{i}}+h(w) w_{x_{i}}\right)^{2}= \\
& =h^{2}(w)-\frac{1}{|\nabla w|^{2}} \sum_{i}\left(J_{x_{i}}^{2}+2 J_{x_{i}} h(w) w_{x_{i}}+h^{2}(w) w_{x_{i}}^{2}\right) \leq \\
& \leq \frac{2 h(w)}{|\nabla w|^{2}} \sum_{i} J_{x_{i}} w_{x_{i}}= \\
& =\left(\frac{2 h(w)}{|\nabla w|^{2}} \nabla w\right) \cdot(\nabla J),
\end{aligned}
$$

isto é,

$$
J_{t}-\Delta J-b \nabla J \leq 0
$$

onde $b$ é contínua onde $\nabla w \neq 0$.

Veja que se $\left(x_{0}, t_{0}\right) \in \Omega_{t_{0}}$ é tal que $\nabla w\left(x_{0}, t_{0}\right)=0$ então, usando (4.56),

$$
J\left(x_{0}, t_{0}\right)=-H\left(w\left(x_{0}, t_{0}\right)\right)=-\int_{0}^{u_{0}-u\left(x_{0}, t_{0}\right)} f\left(u_{0}-s\right) d s \leq 0,
$$


uma vez que como $u_{0} \geq u\left(x_{0}, t_{0}\right)$ temos $u_{0}-s \geq 0$, para todo $s \in\left[0, u_{0}-u\left(x_{0}, t_{0}\right)\right]$ e então $f\left(u_{0}-s\right) \geq 0$, para todo $s \in\left[0, u_{0}-u\left(x_{0}, t_{0}\right)\right]$. Portanto, $J \leq 0$ em $\Omega_{t_{0}} \cap\{(x, t): \nabla w(x, t)=0\}$. Provemos agora que $J \leq 0$ em $\Omega_{t_{0}} \cap\{(x, t): \nabla w(x, t) \neq 0\}$. Sobre $t=0$, segue de (4.53) que

$$
H(w)=H\left(u_{0}-\phi\right)=\tilde{f}\left(u_{0}\right)-\tilde{f}(\phi) \geq \frac{1}{2}|\nabla \phi|^{2}
$$

e então de (4.56) temos que $J(x, 0) \leq 0$. Seja $(y, s)$ um ponto qualquer em $\partial \Omega \times\left(0, t_{0}\right)$ e denote por $\nu$ o vetor normal a $\partial \Omega$ em $y$ apontando para fora de $\Omega$. Se provarmos que

$$
\frac{\partial J}{\partial \nu} \leq 0 \text { em }(y, s)
$$

segue do Lema de Hopof's [6] que $J$ não pode tomar máximo positivo em $(y, s)$ e então como $(y, s)$ é arbitrário, $J$ não pode tomar máximo positivo em $\partial \Omega \times\left(0, t_{0}\right)$. Daí, como $J_{t}-\Delta J-b \nabla J \leq 0$ em $\{(x, t): \nabla w(x, t) \neq 0\}$ com $b$ contínua neste mesmo conjunto, segue do Princípio do Máximo Fraco (Teorema B.1) que

$$
\max _{\bar{\Omega}_{t_{0}} \cap\{(x, t): \nabla w(x, t) \neq 0\}} J=\max _{\Gamma_{t_{0}} \cap\{(x, t): \nabla w(x, t) \neq 0\}} J \leq 0,
$$

donde segue que $J \leq 0$ em $\Omega_{t_{0}} \cap\{(x, t): \nabla w(x, t) \neq 0\}$. Assim, concluímos que $J \leq 0$ em $\Omega_{t_{0}}$ e como

$$
J=\frac{1}{2}|\nabla w|^{2}-H(w)=\frac{1}{2}\left|\nabla\left(u_{0}-u\right)\right|^{2}-H\left(u_{0}-u\right)=\frac{1}{2}|\nabla u|^{2}-\left[\tilde{f}\left(u_{0}\right)-\tilde{f}(u)\right],
$$

a afirmação (4.54) segue.

Para provar (4.68) relembremos o fato de que como $w$ é constante sobre $\partial \Omega$ (para $t=s$ ) então

$$
\Delta w=w_{\nu \nu}+(n-1) k \text { em }(y, s)
$$

onde $k$ é a curvatura média não negativa de $\partial \Omega$ em $y$. Agora veja que

$$
\frac{\partial H}{\partial x_{i}}(w)=h(w) w_{x_{i}}
$$

e então

$$
\nabla H(w)=h(w) \nabla w
$$

donde segue que

$$
\frac{\partial H}{\partial \nu}=h(w) \frac{\partial w}{\partial \nu}
$$

Se $\frac{1}{2}|\nabla w|^{2}=\frac{1}{2} \sum_{i=1}^{n}\left(\frac{\partial w}{\partial x_{i}}\right)^{2}$, então

$$
\frac{\partial}{\partial x_{j}}\left(\frac{1}{2}|\nabla w|^{2}\right)=\sum_{i=1}^{n} \frac{\partial w}{\partial x_{i}} \frac{\partial^{2} w}{\partial x_{j} \partial x_{i}}, \quad \text { para } j=1, \ldots, n
$$


e assim

$$
\nabla\left(\frac{1}{2}|\nabla w|^{2}\right)=\left(\sum_{i=1}^{n} \frac{\partial w}{\partial x_{i}} \frac{\partial^{2} w}{\partial x_{1} \partial x_{i}}, \ldots, \sum_{i=1}^{n} \frac{\partial w}{\partial x_{i}} \frac{\partial^{2} w}{\partial x_{n} \partial x_{i}}\right)
$$

Logo,

$$
\begin{aligned}
\frac{\partial}{\partial \nu}\left(\frac{1}{2}|\nabla w|^{2}\right) & =\nabla\left(\frac{1}{2}|\nabla w|^{2}\right) \cdot \nu= \\
& =\left(\sum_{i} \frac{\partial w}{\partial x_{i}} \frac{\partial^{2} w}{\partial x_{1} \partial x_{i}}, \ldots, \sum_{i} \frac{\partial w}{\partial x_{i}} \frac{\partial^{2} w}{\partial x_{n} \partial x_{i}}\right) \cdot \nu= \\
& =\frac{\partial w}{\partial x_{1}}\left[\left(\frac{\partial^{2} w}{\partial x_{1} \partial x_{1}}, \ldots, \frac{\partial^{2} w}{\partial x_{n} \partial x_{1}}\right) \cdot \nu\right]+\ldots+\frac{\partial w}{\partial x_{n}}\left[\left(\frac{\partial^{2} w}{\partial x_{1} \partial x_{n}}, \ldots, \frac{\partial^{2} w}{\partial x_{n} \partial x_{n}}\right) \cdot \nu\right]= \\
& =\left(\frac{\partial w}{\partial x_{1}}, \ldots, \frac{\partial w}{\partial x_{n}}\right) \cdot\left(\left(\frac{\partial^{2} w}{\partial x_{1} \partial x_{1}}, \ldots, \frac{\partial^{2} w}{\partial x_{n} \partial x_{1}}\right) \cdot \nu, \ldots,\left(\frac{\partial^{2} w}{\partial x_{1} \partial x_{n}}, \ldots, \frac{\partial^{2} w}{\partial x_{n} \partial x_{n}}\right) \cdot \nu\right)= \\
& =\nabla w \cdot \nabla\left(\frac{\partial w}{\partial \nu}\right) .
\end{aligned}
$$

Mas observe que como $w^{\prime}=0$ em $\partial \Omega($ para $t=s)$, então $\nabla w$ é normal a $\partial \Omega($ em $(y, s))$ e daí

$$
\nu= \pm \frac{\nabla w}{|\nabla w|} .
$$

Assim, temos

$$
\frac{\partial}{\partial \nu}\left(\frac{1}{2}|\nabla w|^{2}\right)=\nabla w \cdot \nabla(\nabla w \cdot \nu)=\nabla w \cdot \nabla\left(\nabla w \cdot \pm \frac{\nabla w}{|\nabla w|}\right)=\nabla w \cdot \nabla(|\nabla w|) .
$$

E por outro lado,

$$
\begin{aligned}
\frac{\partial w}{\partial \nu} \frac{\partial^{2} w}{\partial \nu^{2}} & =[\nabla w \cdot \nu]\left[\frac{\partial}{\partial \nu}(\nabla w \cdot \nu)\right]= \\
& =\left[\nabla w \cdot \pm \frac{\nabla w}{|\nabla w|}\right]\left[\nabla\left(\nabla w \cdot \pm \frac{\nabla w}{|\nabla w|}\right) \cdot \pm \frac{\nabla w}{|\nabla w|}\right]= \\
& =\nabla w \cdot\left[ \pm \frac{\nabla w}{|\nabla w|} \cdot \nabla(|\nabla w|) \cdot \pm \frac{\nabla w}{|\nabla w|}\right]= \\
& =\nabla w \cdot \nabla(|\nabla w|)
\end{aligned}
$$

Portanto, em $(y, s)$ temos

$$
\frac{\partial}{\partial \nu}\left(\frac{1}{2}|\nabla w|^{2}\right)=\frac{\partial w}{\partial \nu} \frac{\partial^{2} w}{\partial \nu^{2}}
$$

Segue então de (4.70) e (4.71) que em $(y, s)$

$$
\frac{\partial J}{\partial \nu}=\frac{\partial w}{\partial \nu} \frac{\partial^{2} w}{\partial \nu^{2}}-h(w) \frac{\partial w}{\partial \nu}
$$

e usando (4.69)

$$
\begin{aligned}
\frac{\partial J}{\partial \nu} & =\frac{\partial w}{\partial \nu}[\Delta w-(n-1) k]-h(w) \frac{\partial w}{\partial \nu}=\frac{\partial w}{\partial \nu}[\Delta w-(n-1) k-h(w)]= \\
& =\frac{\partial w}{\partial \nu}\left[w_{t}-(n-1) k\right]=-(n-1) k \frac{\partial w}{\partial \nu}=(n-1) k \frac{\partial u}{\partial \nu} \leq 0,
\end{aligned}
$$


onde também usamos o fato de que $w_{t}=\Delta w-h(w)$ e que $w=u_{0}$ em $\partial \Omega$, implicando que $w_{t}=0$ em $\partial \Omega$.

Portanto, $\frac{\partial J}{\partial \nu} \leq 0$ em $(y, s)$ e (4.68) segue, o que termina a prova.

O Teorema 4.3 pode ser usado para obter limites inferiores de $u$, ou sobre normas de $u$, quando $t \rightarrow T^{-}$. Ilustramos isto no caso especial de (4.36), provando:

Teorema 4.4 Para a solução de (4.36), (4.2), (4.3),

$$
\liminf _{t \rightarrow T^{-}}\|u(\cdot, t)\|_{L^{q}(\Omega)}=+\infty \text { se } q>\frac{n(p-1)}{2} .
$$

Prova. Para cada $t_{0} \in(0, T)$ seja $u_{0}=u\left(x_{0}, t_{0}\right)=\max _{x \in \bar{\Omega}} u\left(x, t_{0}\right)$ e introduzida coordenadas polares $(r, \theta)$ em torno de $x_{0}$. Como $u=0$ sobre $\partial \Omega$, e $u\left(x_{0}, t_{0}\right)=u_{0}$, segue do Teorema do Valor Intermediário que em qualquer direção $\theta$ existe um menor valor de $r, r=r_{0}(\theta)$, tal que

$$
u\left(r, \theta, t_{0}\right)=\frac{1}{2} u_{0}
$$

Denotemos a região $\left\{\left(r, \theta, t_{0}\right): 0 \leq r \leq r_{0}(\theta), \theta \in[0,2 \pi]\right\} \subset \Omega \times\left\{t_{0}\right\}$ em torno de $x_{0}$ por $S_{\left(x_{0}, t_{0}\right)}$. Agora, segue de (4.5) que se $U(t)=\max _{x \in \bar{\Omega}} u(x, t), 0<t<T$ então

$$
\lim _{t \rightarrow T^{-}} U(t)=+\infty
$$

e assim, como

$$
0<K=\max _{\bar{\Omega}}\left(\tilde{f}(\theta)+\frac{1}{2}|\nabla \phi|^{2}\right)<\infty
$$

e $\tilde{f}$ é crescente, se $t_{0}$ for suficientemente próximo de $T$, teremos $u_{0}=U\left(t_{0}\right)$ suficientemente grande e

$$
\tilde{f}\left(u_{0}\right) \geq K>\tilde{f}(\theta)+\frac{1}{2}|\nabla \phi|^{2} \text { em } \Omega .
$$

Desta forma, segue do Teorema 4.3 que

$$
\frac{1}{2}|\nabla u|^{2} \leq \tilde{f}\left(u_{0}\right)-\tilde{f}(u) \text { em } S_{\left(x_{0}, t_{0}\right)}
$$

para cada $t_{0} \in(0, T)$. Mais, em (4.36) temos

$$
\tilde{f}\left(u_{0}\right)=\int_{0}^{u_{0}} \tau^{p} d \tau=\frac{u_{0}^{p+1}}{p+1}
$$

e

$$
\tilde{f}(u)=\int_{0}^{u} \tau^{p} d \tau=\frac{u^{p+1}}{p+1},
$$

e então como $|\nabla u|=u_{r}$ em $S_{\left(x_{0}, t_{0}\right)}$, voltando em (4.73) temos para cada $0<t_{0}<T$,

$$
u_{r}^{2} \leq \frac{2}{p+1}\left[u_{0}^{p+1}-u^{p+1}\right] \text { em } S_{\left(x_{0}, t_{0}\right)} .
$$


Segue então do Teorema do Valor Médio que existe $\xi$ entre $x$ e $x_{0}$ tal que

$$
u_{0}^{p+1}-u^{p+1} \leq(p+1) u^{p}\left(\xi, t_{0}\right)\left[u_{0}-u\right]
$$

e então como $u_{0}=\max _{x \in \bar{\Omega}} u\left(x, t_{0}\right)$ temos

$$
u_{r}^{2} \leq 2 u_{0}^{p}\left(u_{0}-u\right) \text { em } S_{\left(x_{0}, t_{0}\right)}
$$

ou ainda,

$$
\frac{\left|u_{r}\right|}{\sqrt{u_{0}-u}} \leq \sqrt{2} u_{0}^{\frac{p}{2}} \leq 2 u_{0}^{\frac{p}{2}} .
$$

Logo,

$$
\frac{-u_{r}}{\sqrt{u_{0}-u}} \leq 2 u_{0}^{\frac{p}{2}} \quad \text { em } \quad S_{\left(x_{0}, t_{0}\right)}
$$

e então

$$
\int_{0}^{r} \frac{-u_{r}}{\sqrt{u_{0}-u}} d r \leq\left.\int_{0}^{r} 2 u_{0}^{\frac{p}{2}} d r \Rightarrow 2 \sqrt{u_{0}-u}\right|_{0} ^{r} \leq 2 u_{0}^{\frac{p}{2}} r .
$$

Daí, como $u\left(0, \theta, t_{0}\right)=u\left(x_{0}, t_{0}\right)=u_{0}$, temos $\sqrt{u_{0}-u}=0$ para $r=0$ e então

$$
2 \sqrt{u_{0}-u} \leq 2 u_{0}^{\frac{p}{2}} r
$$

isto é,

$$
\sqrt{u_{0}-u} \leq 2 u_{0}^{\frac{p}{2}} r \quad \text { em } \quad S_{\left(x_{0}, t_{0}\right)}
$$

Tomando $r=r_{0}(\theta)$, temos $u\left(r, \theta, t_{0}\right)=\frac{1}{2} u_{0}$ e então $\sqrt{u_{0}-u}=\sqrt{\frac{1}{2} u_{0}}$ e usando (4.74) temos

$$
r_{0}(\theta) \geq \frac{1}{2 \sqrt{2}} \frac{1}{u_{0}^{\frac{p-1}{2}}} \geq \frac{1}{4 u_{0}^{\frac{p-1}{2}}} .
$$

Assim, para cada $t_{0}$ em $(0, T)$ temos

$$
\begin{aligned}
\left\|u\left(x, t_{0}\right)\right\|_{L^{q}(\Omega)}^{q} & =\int_{\Omega} u^{q}\left(x, t_{0}\right) d x \geq \int_{S_{\left(x_{0}, t_{0}\right)}} u^{q}\left(x, t_{0}\right) d x= \\
& =\int_{\partial S_{\left(x_{0}, t_{0}\right)}} \int_{\left\{r<r_{0}(\theta)\right\}} u^{q}\left(r, \theta, t_{0}\right) r^{n-1} d r d S_{\left(x_{0}, t_{0}\right)} .
\end{aligned}
$$

Mas $u=\frac{u_{0}}{2}$ sobre $\partial S_{\left(x_{0}, t_{0}\right)}$ e então

$$
\begin{aligned}
\int_{\partial S_{\left(x_{0}, t_{0}\right)}} \int_{\left\{r<r_{0}(\theta)\right\}} & u^{q}\left(r, \theta, t_{0}\right) r^{n-1} d r d S_{\left(x_{0}, t_{0}\right)}= \\
& =\int_{\partial S_{\left(x_{0}, t_{0}\right)}} \int_{\left\{r<r_{0}(\theta)\right\}}\left(\frac{u_{0}}{2}\right)^{q} r^{n-1} d r d S_{\left(x_{0}, t_{0}\right)}= \\
& =\int_{\partial S_{\left(x_{0}, t_{0}\right)}}\left(\frac{u_{0}}{2}\right)^{q} \frac{r_{0}^{n}(\theta)}{n} d S_{\left(x_{0}, t_{0}\right)} \geq \\
& \geq \int_{\partial S_{\left(x_{0}, t_{0}\right)}} d S_{\left(x_{0}, t_{0}\right)}\left[\left(\frac{u_{0}}{2}\right)^{q} \frac{1}{4^{n} u_{0}^{\frac{n(p-1)}{2}}} \frac{1}{n}\right],
\end{aligned}
$$


onde usamos (4.75). Assim, como $S_{\left(x_{0}, t_{0}\right)} \subset \Omega \times\left\{t_{0}\right\}$ : domínio limitado, temos

$$
\int_{\partial S_{\left(x_{0}, t_{0}\right)}} d S_{\left(x_{0}, t_{0}\right)}<\infty
$$

e então existe $C>0$ tal que

$$
\left\|u\left(x, t_{0}\right)\right\|_{L^{q}(\Omega)}^{q} \geq C u_{0}^{q-\frac{n(p-1)}{2}}
$$

e como para cada $t_{0}, u_{0}=\max _{x \in \bar{\Omega}} u\left(x, t_{0}\right)$, usando (4.5) temos que

$$
u_{0} \rightarrow \infty \text { se } t_{0} \rightarrow T
$$

Daí como $q>\frac{n(p-1)}{2}$ segue que

$$
C u_{0}^{q-\frac{n(p-1)}{2}} \rightarrow \infty \text { se } t_{0} \rightarrow T
$$

e usando (4.76), (4.72) segue.

Teorema 4.5 Suponha que (4.28) e (4.30) valem. Admita também que

$$
\frac{\partial \phi}{\partial \nu}<0 \text { sobre } \partial \Omega
$$

onde $\nu$ representa a normal exterior a $\partial \Omega$. Então o conjunto de pontos de blow-up do problema (4.1)-(4.3) é um subconjunto compacto de $\Omega$.

Prova. Tome qualquer ponto $y_{0} \in \partial \Omega$ e suponha por simplicidade que $y_{0}=0$ e que o semiespaço $\left\{x_{1}>0\right\}$ é tangente a $\Omega$ em $y_{0}$. Seja $\Omega_{\alpha}^{+}=\Omega \cap\left\{x_{1}>\alpha\right\}$, onde $\alpha<0$ e $|\alpha|$ é pequeno, e defina também $\Omega_{\alpha}^{-}=\left\{\left(x_{1}, x^{\prime}\right):\left(2 \alpha-x_{1}, x^{\prime}\right) \in \Omega_{\alpha}^{+}\right\}$, a reflexão de $\Omega_{\alpha}^{+}$com respeito ao plano $\left\{x_{1}=\alpha\right\}$, aqui $x^{\prime}=\left(x_{2}, \ldots, x_{n}\right)$.

Considere a função

$$
w(x, t)=u\left(x_{1}, x^{\prime}, t\right)-u\left(2 \alpha-x_{1}, x^{\prime}, t\right),
$$

para $x \in \Omega_{\alpha}^{-}$. Então, usando (4.1), temos

$$
\begin{aligned}
w_{t}-\Delta w & =\left[u_{t}\left(x_{1}, x^{\prime}, t\right)-\Delta u\left(x_{1}, x^{\prime}, t\right)\right]-\left[u_{t}\left(2 \alpha-x_{1}, x^{\prime}, t\right)-\Delta u\left(2 \alpha-x_{1}, x^{\prime}, t\right)\right]= \\
& =f\left(u\left(x_{1}, x^{\prime}, t\right)-f\left(u\left(2 \alpha-x_{1}, x^{\prime}, t\right)\right) .\right.
\end{aligned}
$$

Segue do Teorema do Valor Médio que

$$
f\left(u\left(x_{1}, x^{\prime}, t\right)-f\left(u\left(2 \alpha-x_{1}, x^{\prime}, t\right)\right)=f^{\prime}\left((1-\theta) u\left(x_{1}, x^{\prime}, t\right)+\theta u\left(2 \alpha-x_{1}, x^{\prime}, t\right)\right) w,\right.
$$

para algum $\theta \in(0,1)$. Daí, como $u$ é contínua em $\bar{\Omega} \times[0, T-\delta]$, subconjunto compacto de $\bar{\Omega}_{T}$, para cada $\delta>0$, temos que $u(\bar{\Omega} \times[0, T-\delta])$ é limitado em $\mathbb{R}$. Assim, como

$$
\left\{(1-\theta) u\left(x_{1}, x^{\prime}, t\right)+\theta u\left(2 \alpha-x_{1}, x^{\prime}, t\right) ; 0<\theta<1\right\} \subset u(\bar{\Omega} \times[0, T-\delta])
$$


e $f^{\prime}$ é contínua (por (4.4)), temos $f^{\prime}$ função limitada em $\left\{(1-\theta) u\left(x_{1}, x^{\prime}, t\right)+\theta u\left(2 \alpha-x_{1}, x^{\prime}, t\right) ; 0<\right.$ $\theta<1\}$. Portanto,

$$
w_{t}-\Delta w=\psi w,
$$

onde $\psi$ é uma função limitada. Além disso, $w=0$ sobre $\left\{x_{1}=\alpha\right\}$ e

$$
w=u\left(x_{1}, x^{\prime}, t\right)>0 \text { sobre }\left(\partial \Omega_{\alpha}^{-} \cap\left\{x_{1}<\alpha\right\}\right) \times(0, T),
$$

uma vez que se $\left(x_{1}, x^{\prime}\right) \in \partial \Omega_{\alpha}^{-} \cap\left\{x_{1}<\alpha\right\}$ então, da definição de $\Omega_{\alpha}^{-}$, temos $\left(2 \alpha-x_{1}, x^{\prime}\right) \in$ $\partial \Omega_{\alpha}^{+} \subset \partial \Omega$. Daí, como $u=0$ sobre $\partial \Omega$, temos

$$
u\left(2 \alpha-x_{1}, x^{\prime}\right)=0, \quad t \in(0, T) .
$$

Logo, como $\left(\partial \Omega_{\alpha}^{-} \cap\left\{x_{1}<\alpha\right\}\right) \subset \Omega$ e $u>0$ em $\Omega \times(0, T)$ (por (4.9)) temos

$$
w=u\left(x_{1}, x^{\prime}, t\right)>0 \text { sobre }\left(\partial \Omega_{\alpha}^{-} \cap\left\{x_{1}<\alpha\right\}\right) \times(0, T) .
$$

Finalmente, se $\alpha$ é suficientemente pequeno, então segue de (4.77) e da compacidade da $\partial \Omega$ que

$$
\phi\left(x_{1}, x^{\prime}\right)>\phi\left(2 \alpha-x_{1}, x^{\prime}\right)
$$

para $\left(x_{1}, x^{\prime}\right) \in \Omega_{\alpha}^{-}$, donde segue que

$$
w(x, 0)>0 \text {, para } x \in \Omega_{\alpha}^{-} .
$$

Então, se $\Gamma_{T}^{-}$denota o bordo parabólico de $\Omega_{\alpha}^{-} \times(0, T)$, temos

$$
\min _{\Gamma_{T}^{-}} w(x, t)=0
$$

Aplicando o Princípio do Mínimo (Teorema B.5) a (4.78) temos que

$$
w \geq 0 \text { em } \Omega_{\alpha}^{-} \times(0, T]
$$

Daí, se existe $\left(x_{0}, t_{0}\right) \in \Omega_{\alpha}^{-} \times(0, T]$ tal que $w\left(x_{0}, t_{0}\right)=0$, segue do Princípio do Mínimo Forte (Teorema B.6) que

$$
w=0 \text { em } \Omega_{\alpha}^{-} \times\left(0, t_{0}\right]
$$

Mas, como vimos, $w(x, 0)>0$ para $x \in \Omega_{\alpha}^{-}$, contradizendo a continuidade de $w$. Concluímos então que $w>0$ em $\Omega_{\alpha}^{-} \times(0, T]$. Assim, veja então que, como $w=0$ sobre $\left\{x_{1}=\alpha\right\}$, se $e_{1}=(1,0, \ldots, 0) \in \mathbb{R}^{n}$ temos que

$$
\lim _{s \rightarrow 0^{-}} \frac{w\left(\left(\alpha, x^{\prime}\right)+s e_{1}, t\right)-w\left(\alpha, x^{\prime}, t\right)}{s}=\lim _{s \rightarrow 0^{-}} \frac{w\left(\left(\alpha, x^{\prime}\right)+s e_{1}, t\right)}{s} \leq 0,
$$


isto é,

$$
\frac{\partial w}{\partial x_{1}} \leq 0 \text { sobre }\left\{x_{1}=\alpha\right\}
$$

Mas,

$$
\frac{\partial w}{\partial x_{1}}=2 \frac{\partial u}{\partial x_{1}}
$$

e então temos

$$
\frac{\partial u}{\partial x_{1}} \leq 0 \text { sobre }\left\{x_{1}=\alpha\right\} .
$$

Como $\alpha$ é arbitrário, segue, varrendo $\alpha$, que

$$
\frac{\partial u}{\partial x_{1}} \leq 0 \text { sobre } \Omega_{\alpha_{0}}^{+} \cup\left\{x_{1}=\alpha_{0}\right\}, 0<t<T,
$$

desde que $\alpha_{0}$ seja suficientemente pequeno.

Introduzimos agora a função

$$
J=u_{x_{1}}+c\left(x_{1}-\alpha_{0}\right) F
$$

em $\bar{\Omega}_{\alpha_{0}}^{+} \times[0, T)$, onde $c$ e $F$ são como em (4.19). Veja que

$$
\begin{gathered}
J_{t}=u_{x_{1} t}+c\left(x_{1}-\alpha_{0}\right) F^{\prime}(u) u_{t} ; \\
J_{x_{1}}=u_{x_{1} x_{1}}+c^{\prime}\left(x_{1}-\alpha_{0}\right) F(u)+c\left(x_{1}-\alpha_{0}\right) F^{\prime}(u) u_{x_{1}},
\end{gathered}
$$

donde segue que

$$
J_{x_{1} x_{1}}=u_{x_{1} x_{1} x_{1}}+c^{\prime \prime}\left(x_{1}-\alpha_{0}\right) F(u)+2 c^{\prime} x_{1}-\alpha_{0} F^{\prime}(u) u_{x_{1}}+c\left(x_{1}-\alpha_{0}\right)\left[F^{\prime \prime}(u) u_{x_{1}}^{2}+F^{\prime}(u) u_{x_{1} x_{1}}\right] ;
$$

e para $2 \leq i \leq n$ temos

$$
J_{x_{i}}=u_{x_{1} x_{i}}+c\left(x_{1}-\alpha_{0}\right) F^{\prime}(u) u_{x_{i}},
$$

donde segue que

$$
J_{x_{i} x_{i}}=u_{x_{1} x_{i} x_{i}}+c\left(x_{1}-\alpha_{0}\right)\left[F^{\prime \prime}(u) u_{x_{i}}^{2}+F^{\prime}(u) u_{x_{i} x_{i}}\right] .
$$

Assim, segue de (4.82) e (4.83) que

$$
\begin{aligned}
\Delta J & =\sum_{i=1}^{n} J_{x_{i} x_{i}}= \\
& =\Delta\left(u_{x_{1}}\right)+c F^{\prime \prime}|\nabla u|^{2}+c F^{\prime} \Delta u+c^{\prime \prime} F+2 c^{\prime} F^{\prime} u_{x_{1}}
\end{aligned}
$$

e usando (4.81) temos

$$
J_{t}-\Delta J=u_{x_{1} t}+c F^{\prime} u_{t}-\Delta\left(u_{x_{1}}\right)-c F^{\prime \prime}|\nabla u|^{2}-c F^{\prime} \Delta u-c^{\prime \prime} F-2 c^{\prime} F^{\prime} u_{x_{1}} .
$$


Veja que como

$$
\frac{\partial}{\partial x_{1}} \Delta u=\Delta u_{x_{1}} \text { e } u_{t}-\Delta u=f
$$

temos que

$$
f^{\prime}(u) u_{x_{1}}=u_{t x_{1}}-\Delta\left(u_{x_{1}}\right)
$$

e voltando em (4.84)

$$
J_{t}-\Delta J=f^{\prime}(u) u_{x_{1}}+c F^{\prime} f-c F^{\prime \prime}|\nabla u|^{2}-c^{\prime \prime} F-2 c^{\prime} F^{\prime} u_{x_{1}} .
$$

Agora, usando o fato de que $u_{x_{1}}=J-c F$ em $\Omega_{\alpha_{0}}^{+} \times(0, T)$, temos

$$
J_{t}-\Delta J=f^{\prime} J+c\left[F^{\prime} f-f^{\prime} F\right]-c F^{\prime \prime}|\nabla u|^{2}-c^{\prime \prime} F-2 c^{\prime} F^{\prime} J+2 c c^{\prime} F^{\prime} F,
$$

donde segue que

$$
J_{t}-\Delta J+\left[2 c^{\prime} F^{\prime}-f^{\prime}\right] J=c\left[F^{\prime} f-f^{\prime} F\right]-c F^{\prime \prime}|\nabla u|^{2}-c^{\prime \prime} F+2 c c^{\prime} F^{\prime} F .
$$

Como $u$ é contínua em $\bar{\Omega} \times[0, T-\delta]$, subconjunto compacto de $\bar{\Omega}_{T}$, para cada $\delta>0$, temos que $u(\bar{\Omega} \times[0, T-\delta])$ é limitado em $\mathbb{R}$. Daí, como $c^{\prime}, f^{\prime}$ e $F^{\prime}$ são contínuas (por (4.4) e (4.19)), segue que

$$
b=2 c^{\prime} F^{\prime}-f^{\prime}
$$

é função limitada. Além disso, escolhendo $c\left(x_{1}-\alpha_{0}\right)=\varepsilon\left(x_{1}-\alpha_{0}\right)$ e usando (4.28), temos

$$
\begin{aligned}
c\left[F^{\prime} f-f^{\prime} F\right]-c F^{\prime \prime}|\nabla u|^{2}-c^{\prime \prime} F+2 c c^{\prime} F^{\prime} F & \leq-2 \varepsilon^{2}\left(x_{1}-\alpha_{0}\right) F F^{\prime}-\varepsilon\left(x_{1}-\alpha_{0}\right) F^{\prime \prime}|\nabla u|^{2}+ \\
+ & 2 \varepsilon^{2}\left(x_{1}-\alpha_{0}\right) F^{\prime} F=-\varepsilon\left(x_{1}-\alpha_{0}\right) F^{\prime \prime}|\nabla u|^{2}
\end{aligned}
$$

e então como $x_{1}>\alpha_{0}$ em $\Omega_{\alpha_{0}}^{+}$e $F^{\prime \prime} \geq 0$,

$$
c\left[F^{\prime} f-f^{\prime} F\right]-c F^{\prime \prime}|\nabla u|^{2}+2 c c^{\prime} F^{\prime} F-c^{\prime \prime} F \leq 0
$$

em $\Omega_{\alpha_{0}}^{+} \times(0, T)$. Voltando em (4.85) temos

$$
J_{t}-\Delta J+b J \leq 0
$$

em $\Omega_{\alpha_{0}}^{+} \times(0, T)$, onde $b$ é função limitada. Agora veja que como sobre $\left\{x_{1}=\alpha_{0}\right\}$

$$
c\left(x_{1}-\alpha_{0}\right)=0
$$

$\mathrm{e}$

$$
\frac{\partial u}{\partial x_{1}} \leq 0
$$


por (4.79), temos $J \leq 0$ sobre $\left\{x_{1}=\alpha_{0}\right\}$. Além disso, sobre $\{t=0\}$, isto é, sobre $\Omega_{\alpha_{0}}^{+} \times\{t=0\}$ temos, por (4.77),

$$
u_{x_{1}}<0 .
$$

Portanto,

$$
\max _{\Omega_{\alpha_{0}}^{+} \times\{t=0\}} u_{x_{1}}=l \leq 0 .
$$

Agora, como $\hat{c}\left(x_{1}-\alpha\right)=x_{1}-\alpha$ é função contínua variando num subconjunto limitado da reta, $F$ é contínua e $\overline{\Omega_{\alpha_{0}}^{+} \times\{t=0\}}$ é subconjunto compacto de $\overline{\Omega \times\{t=0\}}$, temos

$$
\frac{\max }{\Omega_{\alpha_{0}}^{+} \times\{t=0\}}\left(x_{1}-\alpha_{0}\right) F=k \geq 0 .
$$

Assim, como

$$
J=u_{x_{1}}+\varepsilon\left(x_{1}-\alpha_{0}\right) F
$$

em $\bar{\Omega}_{\alpha_{0}}^{+} \times[0, T)$, sobre $\bar{\Omega}_{\alpha_{0}}^{+} \times\{t=0\}$ temos

$$
\begin{aligned}
J & <\max _{\Omega_{\alpha_{0}}^{+} \times\{t=0\}} u_{x_{1}}+\varepsilon \frac{\max }{\Omega_{\alpha_{0}}^{+} \times\{t=0\}}\left(x_{1}-\alpha_{0}\right) F \leq \\
& \leq l+\varepsilon k,
\end{aligned}
$$

onde usamos (4.87) e (4.88). Logo, se $k=0$, temos

$$
J<l \leq 0
$$

e se $k>0$, temos

$$
J<l+\varepsilon k \leq 0,
$$

para $\varepsilon>0$ suficientemente pequeno. Portanto,

$$
J \leq 0 \text { sobre } \Omega_{\alpha_{0}}^{+} \times\{t=0\}
$$

Se pudermos mostrar que

$$
J \leq 0 \text { sobre } \Gamma \times(0, T),
$$

onde $\Gamma=\partial \Omega_{\alpha_{0}}^{+} \cap \partial \Omega$, então teremos que

$$
J \leq 0 \text { sobre } \Gamma_{T}^{+},
$$

isto é,

$$
\max _{\Gamma_{T}^{+}} J=0,
$$


onde $\Gamma_{T}^{+}$denota o bordo parabólico de $\Omega_{\alpha_{0}}^{+}$. Daí, se $\bar{J}=\max _{\Gamma_{T}^{+}} J-J$, então $\bar{J} \geq 0$ em $\Gamma_{T}^{+}$e segue de (4.86) que

$$
\bar{J}_{t}-\Delta \bar{J}+b \bar{J}=-J_{t}+\Delta J+b \max _{\Gamma_{T}^{+}} J-b J \geq b \max _{\Gamma_{T}^{+}} J=0
$$

em $\Omega_{\alpha_{0}}^{+} \times(0, T)$, onde $b$ é função limitada. Daí, segue do Princípio do Mínimo (Teorema B.5) que

$$
\bar{J} \geq 0 \mathrm{em} \Omega_{\alpha_{0}}^{+} \times(0, T)
$$

donde segue que

$$
J \leq 0 \text { em } \Omega_{\alpha_{0}}^{+} \times(0, T)
$$

Provemos então (4.89). Como $\Gamma \subset \partial \Omega$ e $u=0$ sobre $\partial \Omega$, temos por (4.19) que

$$
F(u)=F(0)=0
$$

sobre $\Gamma$. Além disso, segue de (4.79) que

$$
u_{x_{1}} \leq 0 \text { sobre } \Gamma \times(0, T) .
$$

Assim,

$$
J=u_{x_{1}}+\varepsilon\left(x_{1}-\alpha\right) F(u)=u_{x_{1}} \leq 0
$$

sobre $\Gamma \times(0, T)$, e (4.89) segue.

Tendo provado (4.90) concluímos em particular que, usando (4.79),

$$
-u_{x_{1}}=\left|u_{x_{1}}\right| \geq \varepsilon\left(x_{1}-\alpha_{0}\right) F(u)
$$

se $x^{\prime}=0, \alpha_{0} \leq x_{1}<0$. De (4.91) temos que, para $\alpha_{0}<x_{1}<y_{1}<0$,

$$
\int_{x_{1}}^{y_{1}} \frac{-u_{x_{1}}(s, 0, t)}{F(u(s, 0, t))} d s \geq \varepsilon \int_{x_{1}}^{y_{1}}\left(s-\alpha_{0}\right) d s .
$$

Mas, se $G$ é como em (4.31), isto é,

$$
G(s)=\int_{s}^{\infty} \frac{d \tau}{F(\tau)}
$$

como admitimos (4.30), segue de (4.32) que

$$
G^{\prime}(s)=-\frac{1}{F(s)}
$$

e então

$$
\frac{\partial}{\partial x_{1}} G(u(s, 0, t))=-\frac{1}{F(u(s, 0, t))} u_{x_{1}}(s, 0, t) .
$$


Logo,

$$
\int_{x_{1}}^{y_{1}} \frac{-u_{x_{1}}(s, 0, t)}{F(u(s, 0, t))} d s=G\left(u\left(y_{1}, 0, t\right)\right)-G\left(u\left(x_{1}, 0, t\right)\right)
$$

e assim de (4.92)

$$
\begin{aligned}
G\left(u\left(y_{1}, 0, t\right)\right)-G\left(u\left(x_{1}, 0, t\right)\right) & \geq\left.\varepsilon\left(\frac{s^{2}}{2}-\alpha_{0} s\right)\right|_{x_{1}} ^{y_{1}}= \\
& =\frac{\varepsilon}{2}\left(y_{1}^{2}-x_{1}^{2}\right)+\varepsilon \alpha_{0}\left(x_{1}-y_{1}\right)= \\
& =\frac{\varepsilon}{2}\left(y_{1}^{2}-2 \alpha_{0} y_{1}-x_{1}^{2}\right)+\varepsilon \alpha_{0} x_{1}> \\
& >\frac{\varepsilon}{2}\left(y_{1}^{2}-2 x_{1} y_{1}-x_{1}^{2}\right)+\varepsilon \alpha_{0} x_{1}= \\
& =\frac{\varepsilon}{2}\left(y_{1}-x_{1}\right)^{2}+\varepsilon \alpha_{0} x_{1}> \\
& >\frac{\varepsilon}{2}\left(y_{1}-x_{1}\right)^{2}= \\
& =\frac{\varepsilon}{2}\left|y_{1}-x_{1}\right|^{2},
\end{aligned}
$$

onde usamos o fato de que $\alpha_{0}<x_{1}<0$. Assim, para $\alpha_{0}<x_{1}<y_{1}<0$ temos

$$
G\left(u\left(y_{1}, 0, t\right)\right)-G\left(u\left(x_{1}, 0, t\right)\right) \geq \frac{\varepsilon}{2}\left|y_{1}-x_{1}\right|^{2} .
$$

Agora veja que como $G(\infty)=0$, segue que

$$
\limsup _{t \uparrow T} u\left(y_{1}, 0, t\right)<\infty
$$

pois se existisse sequência $\left(t_{n}\right)_{n}$ tal que $t_{n} \uparrow T$ e $u\left(y_{1}, 0, t_{n}\right) \stackrel{n \rightarrow \infty}{\longrightarrow} \infty$ então, como $G(\infty)=0$, seguiria de (4.93) que

$$
0>-\lim _{n \rightarrow \infty} G\left(u\left(x_{1}, 0, t_{n}\right)\right) \geq \varepsilon\left|x_{1}-y_{1}\right|^{2}
$$

o que é absurdo.

A prova de (4.79) pode ser ligeiramente modificada para mostrar que

$$
\frac{\partial u}{\partial \nu}<0 \text { em } \Omega_{\alpha_{0}}^{+} \times(0, T)
$$

para qualquer direção $\nu$ próxima suficiente da direção $x_{1}$. Isto nos permite deduzir que

$$
\limsup _{\substack{t \uparrow T \\ x \rightarrow\left(y_{1}, 0\right)}} u(x, t)<\infty, \text { se } \alpha_{0}<y_{1}<0 .
$$

Assim, todo ponto em $\left\{x^{\prime}=0, \alpha_{0}<x_{1}<0\right\}$ não é ponto de blow-up. A prova acima mostra que $\alpha_{0}$ pode ser escolhido independentemente do ponto inicial $y_{0}$ sobre $\partial \Omega$. Portanto, varrendo $y_{0}$ ao longo da $\partial \Omega$, como $\partial \Omega$ é compacta, concluímos que existe uma $\Omega$-vizinhança $\Omega^{\prime}$ da $\partial \Omega$ tal 
que cada ponto $x \in \Omega^{\prime}$ não é ponto de blow-up. Assim, se $B$ denota o conjunto dos pontos de blow-up, temos

$$
B \subset \Omega \backslash \Omega^{\prime}
$$

onde $\Omega \backslash \Omega^{\prime}$ é um subconjunto compacto de $\Omega$. Então, basta mostrar que $B$ é fechado e teremos o resultado. Seja $\left(x_{n}\right)_{n} \subset B$ tal que $x_{n} \stackrel{n \rightarrow \infty}{\longrightarrow} x_{0}$. Queremos mostrar que $x_{0} \in B$. Como $\left(x_{n}\right)_{n} \subset B$, segue da definição 4.1 que, para cada $n$, existem sequências

$$
\left(x_{n}^{m}\right)_{m} \subset \Omega \quad \text { e } \quad\left(t_{n}^{m}\right)_{m} \subset \mathbb{R}
$$

tais que

$$
x_{n}^{m} \stackrel{m \rightarrow \infty}{\longrightarrow} x_{n} \quad, \quad t_{n}^{m} \stackrel{m \rightarrow \infty}{\longrightarrow} T
$$

e

$$
u\left(x_{n}^{m}, t_{n}^{m}\right) \longrightarrow \infty \text { quando } m \rightarrow \infty
$$

Mostremos então que, para algum $n_{0} \in \mathbb{N}$,

$$
x_{n_{0}}^{m} \longrightarrow x_{0} \quad \text { quando } \quad m \rightarrow \infty
$$

e teremos então que $x_{0} \in B$. Seja $\varepsilon>0$. Como $x_{n} \stackrel{n \rightarrow \infty}{\longrightarrow} x_{0}$, existe $n_{0} \in \mathbb{N}$ tal que

$$
\left|x_{n_{0}}-x_{0}\right|<\frac{\varepsilon}{2} .
$$

Além disso, como $x_{n}^{m} \stackrel{m \rightarrow \infty}{\longrightarrow} x_{n}$, para cada $n$, existe $m_{0} \in \mathbb{N}$ tal que

$$
\left|x_{n_{0}}^{m}-x_{n_{0}}\right|<\frac{\varepsilon}{2}, \quad \forall m \geq m_{0} .
$$

Assim,

$$
\begin{aligned}
\left|x_{n_{0}}^{m}-x_{0}\right| & \leq\left|x_{n_{0}}^{m}-x_{n_{0}}\right|+\left|x_{n_{0}}-x_{0}\right|< \\
& <\frac{\varepsilon}{2}+\frac{\varepsilon}{2}= \\
& =\varepsilon, \quad \forall m \geq m_{0},
\end{aligned}
$$

donde segue que

$$
x_{n_{0}}^{m} \longrightarrow x_{0} \quad \text { quando } \quad m \rightarrow \infty .
$$

Portanto, $x_{0} \in B$ e $B$ é fechado, o que termina a prova. 


\subsection{O caso não-simétrico - continuação}

Nesta seção impomos, em adição a (4.51), a restrição que

$$
\Delta \phi+f(\phi) \geq 0 \quad \text { (não identicamente nula) em } \Omega \text {. }
$$

Admitimos que $f$ satisfaz a condição do Teorema 4.5, e então

$$
\text { o conjunto de pontos de blow-up é um subconjunto compacto de } \Omega \text {. }
$$

Admitimos também que para alguma função $F$, com $F \geq 0, F^{\prime} \geq 0, F^{\prime \prime} \geq 0$, temos

$$
f^{\prime} F-f F^{\prime} \geq 0
$$

podemos, por exemplo, escolher $F$ para ser a mesma função como em (4.28).

Para qualquer $\eta>0$, denotamos

$$
\Omega^{\eta}=\{x \in \Omega: \operatorname{dist}(x, \partial \Omega)>\eta\} .
$$

Aplicando o Princípio do Mínimo a $u_{t}$ e usando (4.94) obtemos

$$
u_{t}>0 \text { em } \Omega_{T}
$$

De fato, segue de (4.1) que

$$
u_{t t}=\Delta\left(u_{t}\right)+f^{\prime}(u) u_{t} \text { em } \Omega \times(0, T),
$$

onde $f^{\prime}$ é função limitada, uma vez que $f^{\prime}$ é contínua (por (4.4)) e $u$ é contínua em $\bar{\Omega} \times[0, T-\delta]$, subconjunto compacto de $\bar{\Omega}_{T}$, para cada $\delta>0$, donde segue que $u(\bar{\Omega} \times[0, T-\delta])$ é limitado em $\mathbb{R}$. Além disso, como $u_{t}$ é contínua em $\Omega \times[0, T)$, para $x \in \Omega$, temos

$$
u_{t}(x, 0)=\lim _{s \rightarrow 0} u_{t}(x, s)
$$

Então de (4.1) temos

$$
u_{t}(x, 0)=\lim _{s \rightarrow 0}[\Delta u(x, s)+f(u(x, s))]
$$

e da continuidade de $\Delta u$ e $f$, usando (4.2) temos

$$
u_{t}(x, 0)=\Delta \phi(x)+f(\phi(x)) \text {, }
$$

para $x \in \Omega$. Veja também que de (4.3) temos

$$
u_{t}(x, t)=0, \quad x \in \partial \Omega, \quad 0<t<T .
$$


Assim, de (4.94), (4.98), (4.99) e (4.100), temos que $u_{t}$ é solução do seguinte problema de valor de bordo inicial:

$$
\left\{\begin{array}{rll}
u_{t t}=\Delta\left(u_{t}\right)+f^{\prime}(u) u_{t}, & \text { em } & \Omega \times(0, T) \\
u_{t}(x, 0)=\Delta \phi(x)+f(\phi(x)) \geq 0, & \text { se } & x \in \Omega \\
u_{t}(x, t)=0, & \text { se } & x \in \partial \Omega, 0<t<T
\end{array}\right.
$$

Temos então que

$$
\min _{\Gamma_{T}} u_{t}=0
$$

e do Princípio do Mínimo Fraco (Teorema B.5) segue que

$$
u_{t} \geq 0 \text { em } \Omega \times(0, T] .
$$

Mas veja que se existe $\left(x_{0}, t_{0}\right) \in \Omega_{T}$ tal que

$$
u_{t}\left(x_{0}, t_{0}\right)=0,
$$

segue do Princípio do Mínimo Forte (Teorema B.6) que

$$
u_{t}=0 \mathrm{em} \Omega_{t_{0}}=\Omega \times\left(0, t_{0}\right] .
$$

Assim, como $u_{t}(x, 0) \geq 0$ (não identicamente nula) para $x \in \Omega$, temos uma contradição com a continuidade de $u_{t}$. Portanto,

$$
u_{t}>0 \text { em } \Omega_{T} .
$$

Obteremos agora uma melhor estimativa inferior a partir do bordo parabólico de $\Omega_{T}$.

Lema 4.3 Se u é solução de (4.36), (4.2), (4.3) então, assumindo (4.94)-(4.96), para qualquer $\eta>0$ existe um $\delta>0$ tal que

$$
u_{t} \geq \delta F(u) \quad \text { em } \quad \Omega^{\eta} \times(\eta, T) .
$$

Prova. Considere a função

$$
J=u_{t}-\delta F(u) .
$$

Veja que

$$
J_{t}=u_{t t}-\delta F^{\prime}(u) u_{t}
$$

e que para $i=1, \ldots, n$,

$$
\frac{\partial J}{\partial x_{i}}=u_{t x_{i}}-\delta F^{\prime}(u) u_{x_{i}},
$$


donde segue que

$$
\frac{\partial^{2} J}{\partial x_{i}{ }^{2}}=u_{t x_{i} x_{i}}-\delta\left[F^{\prime \prime}(u) u_{x_{i}}^{2}+F^{\prime}(u) u_{x_{i} x_{i}}\right]
$$

e então

$$
\begin{aligned}
\Delta J & =\sum_{i=1}^{n} \frac{\partial^{2} J}{\partial x_{i}{ }^{2}}= \\
& =\Delta\left(u_{t}\right)-\delta F^{\prime \prime}|\nabla u|^{2}-\delta F^{\prime} \Delta u
\end{aligned}
$$

De (4.102) e (4.103) temos

$$
\begin{aligned}
J_{t}-\Delta J & =u_{t t}-\delta F^{\prime} u_{t}-\Delta\left(u_{t}\right)+\delta F^{\prime \prime}|\nabla u|^{2}+\delta F^{\prime} \Delta u= \\
& =f^{\prime} u_{t}-\delta F^{\prime} f+\delta F^{\prime \prime}|\nabla u|^{2},
\end{aligned}
$$

onde usamos o fato de que $u_{t}-\Delta u=f$ e então que $u_{t t}-\Delta\left(u_{t}\right)=f^{\prime}(u) u_{t}$. Daí, como $u_{t}=\delta F+J$,

$$
\begin{aligned}
J_{t}-\Delta J & =f^{\prime}[\delta F+J]-\delta F^{\prime} f-\delta F^{\prime \prime}|\nabla u|^{2}= \\
& =f^{\prime} J+\delta\left[f^{\prime} F-f F^{\prime}\right]+\delta F^{\prime \prime}|\nabla u|^{2},
\end{aligned}
$$

e como $F^{\prime \prime} \geq 0$ e $\delta>0$, usando (4.96) segue que

$$
J_{t}-\Delta J-f^{\prime} J=\delta\left[f^{\prime} F-f F^{\prime}\right]+\delta F^{\prime \prime}|\nabla u|^{2} \geq 0,
$$

onde $f^{\prime}$ é função limitada, uma vez que $f^{\prime}$ é contínua (por (4.4)) e $u$ é contínua em $\bar{\Omega} \times[0, T-\delta]$, subconjunto compacto de $\bar{\Omega}_{T}$, para cada $\delta>0$, donde segue que $u(\bar{\Omega} \times[0, T-\delta])$ é limitado em $\mathbb{R}$.

A seguir veja que, em vista de (4.95), se $\eta$ é suficientemente pequeno, temos

$$
\max _{\partial \Omega^{\eta} \times(0, T)}|u|<\infty
$$

e então

$$
0 \leq u \leq \max _{\partial \Omega^{\eta} \times(0, T)}|u|<\infty \text { sobre } \partial \Omega^{\eta} \times(0, T) .
$$

Além disso, $u$ é limitada em $\Omega^{\eta} \times\{\eta\}$ se $\eta$ é suficientemente pequeno $(\eta \ll T)$. Portanto, como $F$ é contínua, temos que, se $\eta$ é suficientemente pequeno,

$$
F(u) \leq C_{0}<\infty
$$

sobre $\partial \Omega^{\eta} \times(0, T)$ e em $\Omega^{\eta} \times\{\eta\}$, isto é, sobre o bordo parabólico de $\Omega^{\eta} \times(\eta, T)$, aqui denotado por $\partial_{\text {parab }}\left(\Omega^{\eta} \times(\eta, T)\right)$. Agora veja que, como $u_{t}>0$ em $\Omega_{T}$ (por (4.97)) e o bordo parabólico de $\Omega^{\eta} \times(\eta, T)$ é um compacto contido em $\Omega_{T}$, temos

$$
c_{0}=\min _{\partial_{\mathrm{parab}}\left(\Omega^{\eta} \times(\eta, T)\right)} u_{t}>0
$$


donde segue que $u_{t} \geq c_{0}>0$ sobre o bordo parabólico de $\Omega^{\eta} \times(\eta, T)$.

Assim, sobre o bordo parabólico de $\Omega^{\eta} \times(\eta, T)$, temos

$$
J=u_{t}-\delta F(u) \geq c_{0}-\delta C_{0}>0
$$

se $0<\delta<\frac{c_{0}}{C_{0}}$, isto é, desde que $\delta$ seja suficientemente pequeno. Daí, em vista de (4.104), segue do Princípio do Mínimo (Teorema B.5) que $J \geq 0 \mathrm{em} \Omega^{\eta} \times(\eta, T)$, o que conclui a prova.

Mais uma vez, consideramos a função $G$ como em (4.31),

$$
G(s)=\int_{s}^{\infty} \frac{1}{F(\tau)} d \tau
$$

onde $F$ é como no caso (4.36), e admitimos (4.30). Como visto na seção 4.2 ,

$$
G^{\prime}(u)=-\frac{1}{F(u)}
$$

e então

$$
-(G(u))_{t}=\frac{1}{F(u)} u_{t}
$$

Mas, segue de (4.101) que, para algum $\delta>0$,

$$
\frac{1}{F(u)} u_{t} \geq \delta \text { em } \Omega_{T}
$$

e então

$$
-(G(u))_{t} \geq \delta \text { em } \Omega_{T},
$$

donde segue que

$$
-\int_{t}^{T}(G(u))_{t} d t \geq \int_{t}^{T} \delta d t
$$

e então

$$
G(u(x, t))-G(u(x, T)) \geq \delta(T-t) .
$$

Daí, como $G(u(x, T)) \geq 0$,

$$
G(u(x, t)) \geq \delta(T-t) \text { em } \Omega_{T} .
$$

Isto nos dá uma limitação superior sobre $u(x, t)$ quando $t \uparrow T$. Em particular:

Teorema 4.6 Se u é solução de (4.36), (4.2), (4.3), então

$$
u(x, t) \leq \frac{C}{(T-t)^{\frac{1}{p-1}}} \quad(C>0) \quad \text { em } \Omega_{T} .
$$


Prova. No caso (4.36) temos $F(u)=u^{\gamma}, 1<\gamma<p$, que nos dá

$$
G(u(x, t))=\frac{1}{\gamma-1} \frac{1}{(u(x, t))^{\gamma-1}} \quad \text { em } \Omega_{T} .
$$

Daí, usando (4.106), temos

$$
\frac{1}{\gamma-1} \frac{1}{(u(x, t))^{\gamma-1}} \geq \delta(T-t)
$$

donde segue que em $\Omega_{T}$

$$
\begin{aligned}
u(x, t) & \leq\left(\frac{1}{(\gamma-1) \delta}\right)^{\frac{1}{\gamma-1}} \frac{1}{(T-t)^{\frac{1}{\gamma-1}}}= \\
& =\frac{C}{(T-t)^{\frac{1}{\gamma-1}}}, \quad C>0 .
\end{aligned}
$$

Agora veja que como $1<\gamma<p, C>0$ e $T-t>0$ em $(0, T)$, temos

$$
\frac{C}{(T-t)^{\frac{1}{\gamma-1}}}<\frac{C}{(T-t)^{\frac{1}{p-1}}}
$$

e então

$$
u(x, t) \leq \frac{C}{(T-t)^{\frac{1}{p-1}}} \quad \text { em } \quad \Omega_{T}
$$

como queríamos.

Observação 4.3 Além disso, o Teorema 4.6 nos permite aplicar um resultado de Giga e Kohn [12] e obter o seguinte resultado:

se u é solução de (4.36), (4.2), (4.3) e se $n=1,2$ ou $n \geq 3$ e $p \leq \frac{(n+2)}{(n-2)}$, então em qualquer ponto de blow-up $x$ vale

$$
\tau^{\beta} u(x+\xi, T-t) \longrightarrow c_{0} \quad\left(\beta=\frac{1}{p-1}\right)
$$

uniformemente quando $\tau \downarrow 0,|\xi| \leq C \tau^{1 / 2}$, onde $c_{0}=0$ ou $c_{0}=\beta^{\beta}$.

Concluímos esta seção provando um complemento do Lema 4.3 e do Teorema 4.6, para a função

$$
U(t)=\max _{x \in \bar{\Omega}} u(x, t)
$$

Teorema 4.7 A função $U(t)$ é Lipschitz contínua e

$$
U^{\prime}(t) \leq f(U(t)) \quad \text { q.s. }
$$


Prova. Sejam $U\left(t_{i}\right)=u\left(x_{i}, t_{i}\right) \quad(i=1,2)$ e $h=t_{2}-t_{1}$. Então, como

$$
u\left(x_{1}, t_{1}\right)=\max _{x \in \bar{\Omega}} u\left(x, t_{1}\right)
$$

e

$$
u\left(x_{2}, t_{2}\right)=\max _{x \in \bar{\Omega}} u\left(x, t_{2}\right)
$$

temos

$$
U\left(t_{2}\right)-U\left(t_{1}\right) \geq u\left(x_{1}, t_{2}\right)-u\left(x_{1}, t_{1}\right)
$$

$$
U\left(t_{2}\right)-U\left(t_{1}\right) \leq u\left(x_{2}, t_{2}\right)-u\left(x_{2}, t_{1}\right) .
$$

Considere agora a função $\varphi_{i}(t)=u\left(x_{i}, t\right), 0<t<T, i=1,2$. Do desenvolvimento de Taylor de $\varphi_{i}$ em torno de $t=t_{i}$ temos, para $i=1,2$, e $0<t<T$,

$$
\varphi_{i}(t)=\varphi_{i}\left(t_{i}\right)+\varphi_{i}^{\prime}\left(t_{i}\right)\left(t-t_{i}\right)+r\left(t_{i}\right),
$$

onde $r$ é contínua, $r\left(t_{i}\right)=0$ e

$$
\lim _{t \rightarrow t_{i}} \frac{r\left(t_{i}\right)}{t-t_{i}}=0,
$$

isto é,

$$
r\left(t_{i}\right)=o\left(t-t_{i}\right) .
$$

Assim, como $\varphi_{i}^{\prime}\left(t_{i}\right)=u_{t}\left(x_{i}, t_{i}\right)$, segue de (4.111) que

$$
u\left(x_{1}, t\right)-u\left(x_{1}, t_{1}\right)=u_{t}\left(x_{1}, t_{1}\right)\left(t-t_{1}\right)+o\left(t-t_{1}\right)
$$

$$
u\left(x_{2}, t\right)-u\left(x_{2}, t_{2}\right)=u_{t}\left(x_{2}, t_{2}\right)\left(t-t_{2}\right)+o\left(t-t_{2}\right),
$$

para $0<t<T$. Logo,

$$
u\left(x_{1}, t_{2}\right)-u\left(x_{1}, t_{1}\right)=u_{t}\left(x_{1}, t_{1}\right)\left(t_{2}-t_{1}\right)+o\left(t_{2}-t_{1}\right)
$$

e

$$
u\left(x_{2}, t_{1}\right)-u\left(x_{2}, t_{2}\right)=u_{t}\left(x_{2}, t_{2}\right)\left(t_{1}-t_{2}\right)+o\left(t_{1}-t_{2}\right) .
$$

Daí, como $h=t_{2}-t_{1}$ e $o\left(t_{1}-t_{2}\right)=o\left(t_{2}-t_{1}\right)$, voltando em (4.109) e (4.110) temos que

$$
U\left(t_{2}\right)-U\left(t_{1}\right) \geq h u_{t}\left(x_{1}, t_{1}\right)+o(h)
$$

$$
U\left(t_{2}\right)-U\left(t_{1}\right) \leq h u_{t}\left(x_{2}, t_{2}\right)+o(h),
$$


donde segue que

$$
u_{t}\left(x_{1}, t_{1}\right)+\frac{o(h)}{h} \leq \frac{U\left(t_{2}\right)-U\left(t_{1}\right)}{h} \leq u_{t}\left(x_{2}, t_{2}\right)+\frac{o(h)}{h}
$$

Daí, como $\frac{o(h)}{h} \rightarrow 0$ quando $h \rightarrow 0$, temos que, para $t_{2}$ suficientemente próximo de $t_{1}$,

$$
-\delta<\frac{o(h)}{h}<\delta
$$

para algum $\delta>0$. Voltando em (4.113) temos

$$
u_{t}\left(x_{1}, t_{1}\right)-\delta \leq \frac{U\left(t_{2}\right)-U\left(t_{1}\right)}{h} \leq u_{t}\left(x_{2}, t_{2}\right)+\delta,
$$

para $t_{2} \sim t_{1}$. Assim, como

$$
m=\min _{0 \leq t \leq T}\left[u_{t}\left(x_{1}, t\right)-\delta\right] \leq u_{t}\left(x_{1}, t_{1}\right)-\delta
$$

e

$$
u_{t}\left(x_{2}, t_{2}\right)+\delta \leq \max _{0 \leq t \leq T}\left[u_{t}\left(x_{2}, t\right)-\delta\right]=M,
$$

temos

$$
m \leq \frac{U\left(t_{2}\right)-U\left(t_{1}\right)}{h} \leq M, \quad \text { se } \quad t_{2} \sim t_{1} .
$$

Assim, se $K=\max \{|m|,|M|\}>0$, temos que

$$
\left|U\left(t_{2}\right)-U\left(t_{1}\right)\right| \leq K\left|t_{2}-t_{1}\right| \quad \text { se } \quad t_{2} \sim t_{1},
$$

donde segue que $U$ é Lipschitz contínua.

Além disso, se $t_{2}>t_{1}$, segue de (4.112) que

$$
\frac{U\left(t_{2}\right)-U\left(t_{1}\right)}{t_{2}-t_{1}} \leq u_{t}\left(x_{2}, t_{2}\right)+o(1)
$$

e como $\Delta u\left(x_{2}, t_{2}\right) \leq 0$, uma vez que $u\left(x_{2}, t_{2}\right)=\max _{x \in \bar{\Omega}} u\left(x, t_{2}\right)$, segue de (4.1) que

$$
u_{t}\left(x_{2}, t_{2}\right) \leq f\left(u\left(x_{2}, t_{2}\right)\right)=f\left(U\left(t_{2}\right)\right) .
$$

Consequentemente, se $t$ é ponto de diferenciabilidade de $U$, com $U(t)=u(x, t)$, temos que

$$
\lim _{h \rightarrow 0} \frac{U(t+h)-U(t)}{h} \quad \text { existe }
$$

e que

$$
\begin{aligned}
\lim _{h \rightarrow 0} \frac{U(t+h)-U(t)}{h} & =\lim _{h \rightarrow 0^{+}} \frac{U(t+h)-U(t)}{h} \leq \\
& \leq \lim _{h \rightarrow 0^{+}}\left[u_{t}(x, t)+o(1)\right] \leq \\
& \leq \lim _{h \rightarrow 0^{+}}[f(U(t))+o(1)]= \\
& =\lim _{h \rightarrow 0^{+}} f(U(t))= \\
& =f(U(t)),
\end{aligned}
$$


uma vez que $o(1) \rightarrow 0$ quando $h \rightarrow 0$. Portanto, em qualquer ponto de diferenciabilidade $t$ de $U$, temos

$$
U^{\prime}(t) \leq f(U(t))
$$

Daí, como $U$ é Lipschitz contínua, $U$ é diferenciável quase sempre e então (4.108) segue.

Agora, admitindo (4.40) e introduzida a notação (4.41) temos, como visto em (4.42),

$$
-(\hat{f}(U(t)))_{t}=\frac{1}{f(U(t))} U^{\prime}(t)
$$

e podemos então reescrever (4.108) na forma

$$
-(\hat{f}(U(t)))_{t} \leq 1
$$

ou, por integração

$$
\hat{f}(U(t)) \leq T-t
$$

Isto é um complemento de (4.106), que nos dá:

Corolário 4.1 Se u é uma solução de (4.36), (4.2), (4.3), então

$$
\max _{x \in \bar{\Omega}} u(x, t) \geq \frac{C}{(T-t)^{\frac{1}{p-1}}} \quad(C>0),
$$

para $0<t<T$.

Prova. Em (4.36) temos $f(\tau)=\tau^{p}, p>1$ e então como visto em (4.46)

$$
\hat{f}(U(t))=\frac{1}{(p-1)(U(t))^{(p-1)}}, \quad \text { para } 0<t<T .
$$

Segue então de (4.114) que

$$
\frac{1}{(p-1)(U(t))^{(p-1)}} \leq T-t \quad \text { para } 0<t<T,
$$

donde segue que

$$
\begin{aligned}
U(t) & \geq \frac{1}{(p-1)^{\frac{1}{p-1}}(T-t)^{\frac{1}{p-1}}}= \\
& =\frac{C}{(T-t)^{\frac{1}{p-1}}},
\end{aligned}
$$

para $C>0$ e $0<t<T$, isto é,

$$
\max _{x \in \bar{\Omega}} u(x, t) \geq \frac{C}{(T-t)^{\frac{1}{p-1}}},
$$

para $C>0$ e $0<t<T$. 


\section{Apêndice A}

\section{O espectro do Laplaciano}

\section{A.1 O operador solução}

Dado $f \in L^{2}(\Omega)$, existe uma única solução $u \in H_{0}^{1}(\Omega)$ do problema

$$
\left\{\begin{aligned}
-\Delta u & =f, \text { em } \Omega \\
u & =0, \text { sobre } \partial \Omega
\end{aligned}\right.
$$

pois, sendo $f \in L^{2}(\Omega)$, considere

$$
\begin{aligned}
F: H_{0}^{1}(\Omega) & \longrightarrow \mathbb{R} \\
\phi & \longmapsto F(\phi)=\int_{\Omega} f \phi d x
\end{aligned}
$$

que é linear e contínuo, isto é,

$$
|F(\phi)| \leq \int_{\Omega}|f||\phi| d x
$$

Por Hölder obtemos

$$
|F(\phi)| \leq\|f\|_{L^{2}}\|\phi\|_{L^{2}}
$$

e da desigualdade de Poincaré

$$
|F(\phi)| \leq C\|f\|_{L^{2}}\|\phi\|_{H_{0}^{1}}
$$

o que implica,

$$
|F(\phi)| \leq M\|\phi\|_{H_{0}^{1}} \quad ; \quad M=C\|f\|_{L^{2}}
$$

mostrando a continuidade.

Logo, $F \in H^{-1}(\Omega)=\left(H_{0}^{1}(\Omega)\right)^{*}$ e pelo Teorema da Representação de Riesz, existe um único $u \in H_{0}^{1}(\Omega)$ tal que

$$
(u, \phi)_{H_{0}^{1}(\Omega)}=F(\phi), \quad \forall \phi \in H_{0}^{1}(\Omega),
$$


de onde segue

$$
\int_{\Omega} \nabla u \nabla \phi d x=\int_{\Omega} f \phi d x, \forall \phi \in H_{0}^{1}(\Omega),
$$

ou seja, $u$ é a única solução fraca do problema $\left(P_{1}\right)$.

Pelo que foi visto até o momento, podemos definir o operador solução dado por

$$
\begin{aligned}
S: L^{2}(\Omega) & \longrightarrow H_{0}^{1}(\Omega) \\
f & \longmapsto S(f)=u,
\end{aligned}
$$

que associa a cada $f \in L^{2}(\Omega)$ a única solução $u=S(f)$ do problema $\left(P_{1}\right)$.

\section{A.2 Propriedades do operador solução}

Nesta seção provaremos algumas propriedades do operador solução $S$ para então obter informações sobre o espectro do operador Laplaciano. Seguem as propriedades:

(P.1) $S$ é linear, isto é,

$$
S(\lambda f+\gamma g)=\lambda S(f)+\gamma S(g), \quad \forall f, g \in L^{2}(\Omega) \text { e } \forall \lambda, \gamma \in \mathbb{R} .
$$

Prova. Sejam $u=S(f)$ e $v=S(g)$. Por definição

$$
\left\{\begin{aligned}
-\Delta u & =f, \text { em } \Omega \\
u & =0, \text { sobre } \partial \Omega
\end{aligned}\right.
$$

e

$$
\left\{\begin{aligned}
-\Delta v & =g, \text { em } \Omega \\
v & =0, \text { sobre } \partial \Omega
\end{aligned}\right.
$$

Logo, multiplicando respectivamente por $\lambda$ e $\gamma$ obtemos

$$
\left\{\begin{aligned}
-\Delta(\lambda u) & =\lambda f, \text { em } \Omega \\
\lambda u & =0, \text { sobre } \partial \Omega
\end{aligned}\right.
$$

$\mathrm{e}$

$$
\left\{\begin{aligned}
-\Delta(\gamma v) & =\lambda g, \text { em } \Omega \\
\gamma v & =0, \text { sobre } \partial \Omega
\end{aligned}\right.
$$

o que implica,

$$
\left\{\begin{aligned}
-\Delta(\lambda u+\gamma v) & =(\lambda f+\gamma g), \text { em } \Omega \\
\lambda u+\gamma v & =0, \text { sobre } \partial \Omega
\end{aligned}\right.
$$


e consequentemente

$$
S(\lambda f+\gamma g)=\lambda u+\gamma v,
$$

isto é,

$$
S(\lambda f+\gamma g)=\lambda S(f)+\gamma S(g), \quad \forall f, g \in L^{(2)} \Omega \text { e } \forall \lambda, \gamma \in \mathbb{R} .
$$

(P.2) $S$ é contínuo, ou seja, existe $M>0$ verificando

$$
\|S(f)\| \leq M\|f\|_{L^{2}} \quad \forall f \in L^{2}(\Omega)
$$

Prova. Seja $u=S(f)$. Então,

$$
\left\{\begin{aligned}
-\Delta u & =f, \text { em } \Omega \\
u & =0, \text { sobre } \partial \Omega
\end{aligned}\right.
$$

o que implica, por definição

$$
\int_{\Omega} \nabla u \nabla \phi d x=\int_{\Omega} f \phi d x \quad \forall \phi \in H_{0}^{1}(\Omega) .
$$

Escolhendo $\phi=u$, obtemos

$$
\int_{\Omega}|\nabla u|^{2} d x=\int_{\Omega} f u d x
$$

e assim,

$$
\|u\|_{H_{0}^{1}}^{2}=\int_{\Omega} f u d x
$$

de onde segue

$$
\|u\|_{H_{0}^{1}}^{2} \leq \int_{\Omega}|f||u| d x
$$

Por Hölder tem-se

$$
\|u\|_{H_{0}^{1}}^{2} \leq\|f\|_{L^{2}}\|u\|_{L^{2}}
$$

Da desigualdade de Poincaré obtemos

$$
\|u\|_{H_{0}^{1}}^{2} \leq C\|f\|_{L^{2}}\|u\|_{H_{0}^{1}}
$$

Se $u \neq 0$, então

$$
\|u\|_{H_{0}^{1}} \leq C\|f\|_{L^{2}},
$$

isto é,

$$
\|S(f)\| \leq C\|f\|_{L^{2}}, \quad \forall f \in L^{2}(\Omega) .
$$


(P.3) $S$ pode ser vista de $L^{2}(\Omega)$ em $L^{2}(\Omega)$. Além disso, neste caso $S$ é linear e compacto.

Prova. Observe que,

$$
S: L^{2}(\Omega) \longrightarrow H_{0}^{1}(\Omega)
$$

é uma aplicação linear contínua e como a imersão

$$
i: H_{0}^{1}(\Omega) \longrightarrow L^{2}(\Omega)
$$

é compacta, recordando que

(a) composição de aplição linear é linear;

(b) composição de um operador contínuo com um compacto é compacto;

podemos concluir que o operador

$$
S: L^{2}(\Omega) \longrightarrow L^{2}(\Omega)
$$

é linear compacto.

(P.4) O operador $S: L^{2}(\Omega) \longrightarrow L^{2}(\Omega)$ é simétrico, ou seja,

$$
(S(f), g)_{L^{2}}=(f, S(g))_{L^{2}}
$$

onde, $(.)_{L^{2}}$ é o produto interno de $L^{2}(\Omega)$.

Prova. Sejam $u=S(f)$ e $v=S(g)$. Devemos mostrar a seguinte igualdade

$$
\int_{\Omega} u g d x=\int_{\Omega} v f d x
$$

Desde que $u=S(f)$ e $v=S(g)$ temos que

$$
\left\{\begin{aligned}
-\Delta u & =f, \text { em } \Omega \\
u & =0, \text { sobre } \partial \Omega
\end{aligned}\right.
$$

e

$$
\left\{\begin{aligned}
-\Delta v & =g, \text { em } \Omega \\
v & =0, \text { sobre } \partial \Omega
\end{aligned}\right.
$$

Portanto,

$$
\int_{\Omega} \nabla u \nabla \phi d x=\int_{\Omega} f \phi d x, \forall \phi \in H_{0}^{1}(\Omega)
$$




$$
\int_{\Omega} \nabla v \nabla \varphi d x=\int_{\Omega} g \varphi d x, \quad \forall \varphi \in H_{0}^{1}(\Omega)
$$

Fixe $\phi=v$ e $\varphi=u$, obtemos

$$
\int_{\Omega} \nabla v \nabla u d x=\int_{\Omega} f v d x
$$

e

$$
\int_{\Omega} \nabla v \nabla u d x=\int_{\Omega} g u d x
$$

de onde segue

$$
\int_{\Omega} u g d x=\int_{\Omega} v f d x
$$

isto é,

$$
(S(f), g)_{L^{2}}=(f, S(g))_{L^{2}}
$$

(P.5) $S(f)=0$ se, e somente se, $f=0$.

Prova. Considere que $S(f)=u$. Então, $u \in H_{0}^{1}(\Omega)$ é solução do problema

$$
\left\{\begin{aligned}
-\Delta u & =f, \text { em } \Omega \\
u & =0, \text { sobre } \partial \Omega
\end{aligned}\right.
$$

ou seja,

$$
\int_{\Omega} \nabla u \nabla \phi d x=\int_{\Omega} f \phi d x, \quad \forall \phi \in H_{0}^{1}(\Omega)
$$

Se $f=0$, então

$$
\int_{\Omega} \nabla u \nabla \phi d x=0, \quad \forall \phi \in H_{0}^{1}(\Omega) .
$$

Fixando $\phi=u$, temos

$$
\int_{\Omega}|\nabla u|^{2} d x=\|u\|_{H_{0}^{1}}^{2}=0 \quad \Leftrightarrow \quad u=0
$$

isto é,

$$
S(f)=0 .
$$

Supondo que $S(f)=0$, segue de (A.1) que

$$
\int_{\Omega} f \phi d x=0, \quad \forall \phi \in H_{0}^{1}(\Omega)
$$

e então $f=0$. 
(P.6) $\lambda=0$ não é autovalor de $S$.

Prova. Veja que

$$
N(S)=\left\{\varphi \in L^{2}(\Omega) ; S(\varphi)=0\right\}
$$

e por (P.5), temos que

$$
S(\varphi)=0 \Leftrightarrow \varphi=0 .
$$

Logo, $N(S)=0$, mostrando a propriedade.

(P.6) $S$ é positivo, isto é,

$$
(S(f), f)_{L^{2}}>0, \quad \forall f \neq 0 .
$$

Prova. Seja $S(f)=u$ com $u \neq 0$, então

$$
\left\{\begin{aligned}
-\Delta u & =f, \text { em } \Omega \\
u & =0, \text { sobre } \partial \Omega
\end{aligned}\right.
$$

o que implica,

$$
\int_{\Omega} \nabla u \nabla \phi d x=\int_{\Omega} f \phi d x \quad \forall \phi \in H_{0}^{1}(\Omega) .
$$

Fixando $\phi=u$, segue

$$
0<\|u\|_{H_{0}^{1}}^{2}=\int_{\Omega} f u d x=\int_{\Omega} f S(f) d x=(S(f), f)_{L^{2}},
$$

mostrando que

$$
(S(f), f)_{L^{2}}>0, \quad \forall f \neq 0 .
$$

Provadas as propriedades anteriores, usando a Análise Funcional (veja [4]) concluímos que:

Afirmação 1: O operador $S: L^{2}(\Omega) \longrightarrow L^{2}(\Omega)$ tem uma sequência de autovalores reais positivos $\left\{\mu_{n}\right\}$ verificando

$$
\mu_{1}>\mu_{2}>\ldots>\mu_{n}>\ldots>0
$$

com

$$
\lim _{n \rightarrow \infty} \mu_{n}=0
$$

Além disso, temos que: 
Afirmação 2: Os autovalores de $S$ são os inversos dos autovalores de

$$
\left\{\begin{aligned}
-\Delta u & =\lambda u, \text { em } \Omega \\
u & =0, \text { sobre } \partial \Omega
\end{aligned}\right.
$$

De fato, se $\lambda$ é um autovalor de $-\Delta$, então existe $\phi \neq 0$ verificando

$$
\left\{\begin{aligned}
-\Delta \phi & =\lambda \phi, \text { em } \Omega \\
\phi & =0, \text { sobre } \partial \Omega
\end{aligned}\right.
$$

Desde que $\lambda \neq 0$, segue da definição de solução fraca que

$$
\int_{\Omega} \nabla \phi \nabla \psi d x=\int_{\Omega} \lambda \psi \phi d x, \quad \forall \psi \in H_{0}^{1}(\Omega) .
$$

Fixando $\phi=\psi$, tem-se

$$
\|\phi\|_{H_{0}^{1}}^{2}=\lambda\|\phi\|_{L^{2}}^{2},
$$

e consequentemente

$$
\lambda=\frac{\|\phi\|_{H_{0}^{1}}^{2}}{\|\phi\|_{L^{2}}^{2}}>0 .
$$

Assim, considere o problema

$$
\left\{\begin{aligned}
-\Delta\left(\frac{1}{\lambda} \phi\right) & =\phi, \text { em } \Omega \\
\phi & =0, \text { sobre } \partial \Omega
\end{aligned}\right.
$$

de onde segue que,

$$
S(\phi)=\frac{1}{\lambda} \phi,
$$

mostrando que $\frac{1}{\lambda}$ é autovalor de $S$.

Agora, se $\mu>0$ é autovalor de $S$, então existe $\phi \in L^{2}(\Omega), \phi \neq 0$, com

$$
S(\phi)=\mu \phi .
$$

Logo,

$$
\frac{1}{\mu} S(\phi)=\phi
$$

Visto que $S$ é linear, temos

$$
S\left(\frac{1}{\mu} \phi\right)=\phi
$$


de onde concluímos que

$$
\left\{\begin{aligned}
-\Delta \phi & =\frac{1}{\mu} \phi, \text { em } \Omega \\
\phi & =0, \text { sobre } \partial \Omega
\end{aligned}\right.
$$

mostrando que $\frac{1}{\mu}$ é autovalor de $\left(P_{\lambda}\right)$.

Das Afirmações 1 e 2 concluímos que

Afirmação 3: O problema $\left(P_{\lambda}\right)$ possui uma sequência $\left\{\lambda_{n}\right\}$ de autovalores reais positivos verificando

$$
\lambda_{1}<\lambda_{2}<\ldots<\lambda_{n}<\ldots
$$

e

$$
\lim _{n \rightarrow \infty} \lambda_{n}=\infty
$$




\section{Apêndice B}

\section{O Princípio do Máximo}

Apresentamos a seguir resultados (veja [21]) de grande importância na prova dos resultados do Capítulo 4.

Sejam $\Omega$ um aberto limitado em $\mathbb{R}^{n}$ e $\Omega_{T}=\Omega \times(0, T]$, para algum tempo $T>0$ fixado. Seja $\Gamma_{T}=\bar{\Omega}_{T}-\Omega_{T}$ o bordo parabólico de $\Omega_{T}$. Para cada $t$ denotemos por $L$ o operador diferencial parcial de segunda ordem que possui a forma não-divergente

$$
L u=-\sum_{i, j=1}^{n} a^{i j} u_{x_{i} x_{j}}+\sum_{i=1}^{n} b^{i} u_{x_{i}}+c u,
$$

onde os coeficientes $a^{i j}, b^{i}, c$ são contínuos.

\section{B.1 Princípio do Máximo}

Definição B.1 Dizemos que o operador diferencial $\frac{\partial}{\partial t}+L$ é (uniformemente) parabólico se existe uma constante $\theta>0$ tal que

$$
\sum_{i, j=1}^{n} a^{i j}(x, t) \xi_{i} \xi_{j} \geq \theta|\xi|^{2}
$$

para todo $(x, t) \in \Omega_{T}, \xi \in \mathbb{R}^{n}$.

Suporemos sempre que a condição de parabolicidade uniforme definida anteriormente é satisfeita, e também que $a^{i j}=a^{j i}(i, j=1, \ldots, n)$.

Teorema B.1 (Princípio do Máximo Fraco) Suponha que $u \in C_{1}^{2}\left(\Omega_{T}\right) \cap C\left(\bar{\Omega}_{T}\right)$ e que

$$
c \equiv 0 \quad \text { em } \Omega_{T} .
$$


(i) $\mathrm{Se}$

$$
u_{t}+L u \leq 0 \text { em } \Omega_{T},
$$

então

$$
\max _{\Omega_{T}} u=\max _{\Gamma_{T}} u .
$$

(ii) Por outro lado, se

$$
u_{t}+L u \geq 0 \quad \text { em } \Omega_{T},
$$

então

$$
\min _{\Omega_{T}} u=\min _{\Gamma_{T}} u .
$$

Observação B.1 Uma função u satisfazendo a desigualdade (B.2) é chamada de uma subsolução, e então estamos afirmando que uma subsolução atinge seu máximo sobre o bordo parabólico $\Gamma_{T}$. Similarmente, u é uma supersolução desde que (B.3) é satisfeita, e neste caso u atinge seu minimo sobre $\Gamma_{T}$.

Teorema B.2 (Princípio do Máximo Fraco para $c \geq 0$.) Suponha que $u \in C_{1}^{2}\left(\Omega_{T}\right) \cap$ $C\left(\bar{\Omega}_{T}\right)$ e que

$$
c \geq 0 \quad \mathrm{em} \quad \Omega_{T} .
$$

(i) $\mathrm{Se}$

$$
u_{t}+L u \leq 0 \quad \text { em } \Omega_{T},
$$

então

$$
\max _{\bar{\Omega}_{T}} u \leq \max _{\Gamma_{T}} u^{+} .
$$

(ii) $\mathrm{Se}$

$$
u_{t}+L u \geq 0 \quad \text { em } \quad \Omega_{T},
$$

então

$$
\min _{\bar{\Omega}_{T}} u \geq-\max _{\Gamma_{T}} u^{-} .
$$

Observação B.2 Em particular, se $u_{t}+L u=0$ dentro de $\Omega_{T}$ então

$$
\max _{\bar{\Omega}_{T}}|u|=\max _{\Gamma_{T}}|u| .
$$


Teorema B.3 (Princípio do Máximo Forte) Suponha que $u \in C_{1}^{2}\left(\Omega_{T}\right) \cap C\left(\bar{\Omega}_{T}\right)$ e que

$$
c \equiv 0 \quad \text { em } \Omega_{T} .
$$

Suponha também que $\Omega$ é conexo.

(i) $\mathrm{Se}$

$$
u_{t}+L u \leq 0 \quad \text { em } \Omega_{T}
$$

e u atinge seu máximo sobre $\bar{\Omega}_{T}$ em um ponto $\left(x_{0}, t_{0}\right) \in \Omega_{T}$, então

$$
u \text { é constante em } \Omega_{t_{0}}=\Omega \times\left(0, t_{0}\right] \text {. }
$$

(ii) Por outro lado, se

$$
u_{t}+L u \geq 0 \quad \text { em } \Omega_{T}
$$

e u atinge seu mínimo sobre $\bar{\Omega}_{T}$ em um ponto $\left(x_{0}, t_{0}\right) \in \Omega_{T}$, então

$$
u \text { é constante em } \Omega_{t_{0}} \text {. }
$$

Teorema B.4 (Princípio do Máximo Forte para $c \geq 0$.) Suponha que $u \in C_{1}^{2}\left(\Omega_{T}\right) \cap C\left(\bar{\Omega}_{T}\right)$ e que

$$
c \geq 0 \quad \mathrm{em} \quad \Omega_{T} .
$$

Suponha também que $\Omega$ é conexo.

(i) $\mathrm{Se}$

$$
u_{t}+L u \leq 0 \quad \text { em } \Omega_{T}
$$

e u atinge um máximo não-negativo sobre $\bar{\Omega}_{T}$ em um ponto $\left(x_{0}, t_{0}\right) \in \Omega_{T}$, então

$$
u \text { é constante em } \Omega_{t_{0}} \text {. }
$$

(ii) Similarmente, se

$$
u_{t}+L u \geq 0 \quad \text { em } \Omega_{T},
$$

e u atinge um mínimo não-positivo sobre $\bar{\Omega}_{T}$ em um ponto $\left(x_{0}, t_{0}\right) \in \Omega_{T}$, então

$$
u \text { é constante em } \Omega_{t_{0}} .
$$




\section{B.2 Princípio do Mínimo - outra versão}

Apresentamos a seguir uma versão do Princípio do Máximo que não faz uso do sinal do coeficiente do termo linear do operador diferencial.

O Espaço Ordenado $S^{n}$. O conjunto das matrizes $n \times n$ reais simétricas $a=\left(a_{i j}\right)$ é denotado por $S^{n}$. Se $a$ é positiva semidefinida, escrevemos $a \geq 0$,

$$
a \geq 0 \Leftrightarrow \xi^{T} a \xi \equiv \sum_{i, j=1}^{n} a_{i j} \xi_{i} \xi_{j} \geq 0 \text { para todo } \xi \in \mathbb{R}^{n} .
$$

Esta notação gera uma relação de ordem em $S^{n}$ :

$$
\text { para } r, s \in S^{n} \text { escrevemos } r \leq s \text { se } s-r \geq 0 \text {. }
$$

Por exemplo $\left(I_{n}\right.$ denota a matriz unitária $\left.n \times n\right)$,

$$
a \geq \alpha I_{n} \Leftrightarrow \xi^{T} a \xi=\sum_{i, j=1}^{n} a_{i j} \xi_{i} \xi_{j} \geq \alpha \sum_{i, j=1}^{n} \xi^{2} \text { para todo } \xi \in \mathbb{R}^{n} .
$$

Considere a equação linear

$$
u_{t}=\sum_{i, j=1}^{n} a_{i j}(x, t) u_{x_{i} x_{j}}+\sum_{i=1}^{n} b_{i}(x, t) u_{x_{i}}+c(x, t) u+d(x, t),
$$

que é abreviada como

$$
u_{t}=a u_{x x}+b u_{x}+c u+d,
$$

onde $a=\left(a_{i j}\right) \in S^{n}, b=\left(b_{i}\right) \in \mathbb{R}^{n}$. Os coeficientes são definidos em $\Omega_{T}$. Admitimos sempre que $a(x, t) \geq 0$, mas algumas vezes condições mais fortes são necessárias.

Definição B.2 Sejam $x=\left(x_{1}, \ldots, x_{n}\right) \in \mathbb{R}^{n}, t \in \mathbb{R}$ e seja $u(x, t)$ uma função de valor real. $O$ gradiente e o Hessiano de u com respeito a $x$ são denotados respectivamente por $u_{x}$ e $u_{x x}$,

$$
u_{x}=\left(u_{x_{1}}, \ldots, u_{x_{n}}\right), u_{x x}=\left(u_{x_{i} x_{j}}\right) \in S^{n} .
$$

A função $u(x, t): \bar{\Omega}_{T} \rightarrow \mathbb{R}$ é admissível, $u \in Z=Z\left(\Omega_{T}\right)$ se u é contínua em $\bar{\Omega}_{T}$ e $u_{t}, u_{x}, u_{x x}$ são contínuas em $\Omega_{T}$.

Teorema B.5 (Princípio do Mínimo - outra versão) Seja $w \in Z\left(\Omega_{T}\right)$. Então

$w \geq 0$ sobre $\Gamma_{T}$ e $w_{t} \geq a w_{x x}+b w_{x}+c w$ em $\Omega_{T}$ (ou: se $w<0$ )

implica $w \geq 0$ em $\Omega_{T}$, se $\sup c<\infty$. 
Teorema B.6 (Princípio do Mínimo Forte - outra versão) Admita que $w \in Z$ satisfaz

$$
w_{t} \geq a w_{x x}+b w_{x}+c w \quad \text { e } w \geq 0 \text { em } \Omega_{T} .
$$

Pedimos que a,b e c sejam localmente limitadas em $\Omega_{T}$ e que $\xi^{T} a \xi \geq \alpha \xi^{2}(\alpha>0)$ localmente em $\Omega_{T}$. Se $w(\bar{x}, \bar{t})=0$, onde $(\bar{x}, \bar{t}) \in \Omega_{T}$, então $w(x, t)=0$ para $t \leq \bar{t}$. 


\section{Referências bibliográficas}

[1] Amann, H., Linear and Quasilinear Parabolic Problems. Vol. I. Birkhäuser Verlag, Basel, (1995).

[2] Bebernes, J. W., Kassoy, D. R., A mathematical analysis of blow-up for thermal reactions-the spatially nonhomogeneous case. SIAM J. APPL. MATH., 40 (1981), 476-484.

[3] Brandle, C., Brunner, H., Blow-up in diffusion equations: A survey. J. Comp. Appl. Math., 97 (1998), 3-22.

[4] Brézis, H., Análisis funcional. Teoría y aplicaciones. Alianza Editorial S. A., Madrid, (1984).

[5] Chipot, M., Some blow-up results for a nonlinear parabolic problem with a gradient term. SIAM J. Math. Anal., 20 (1989), 886-907.

[6] Evans, L. C., Partial Differential Equations. Second Edition. American Mathematical Society. New York, 19 (2000).

[7] Evgrafov, M. A., Asymptotic estimates and entire functions. Gordon and Breach., Inc., New York, (1961). Transled by Allen L. Shields. Russian Tracts on Avanced Mathematics and Physics, Vol. IV.

[8] Friedman, A., McLeod, B., Blow-up of Positive Solutions of Semilinear Heat Equations. Indiana Univ. Math. J., 34 (1985), 425-447.

[9] Fujita, H., On the blow-up of solutions of the Cauchy problem for $u_{t}=\Delta u+u^{1+\alpha}$. J. Fac. Sci. Univ. Tokyo Sect. IA Math., 13 (1966), 109-124.

[10] Fujita, H., On some nonexistence and nonuniqueness theorems for nonlinear parabolic equations. Proc. Symp. Pure Math., 18 (1968), Part I, Amer. Math. Soc., 138-161.

[11] Galaktionov, V.A., Vásquez, J.L, The problem of blow-up in nonlinear parabolic equations. Discrete and Continuous Dynamical Systems, 8 (2002), 399-433. 
[12] Giga, Y, Kohn, R. V., Asymptotically self-similar blow-up of semilinear heat equations. Communications on Pure and Applied Mathematics, 38 (1985), 297-319.

[13] Hayahawa, K., On nonexistence nonexistence of global solutions of some semilinear parabolic equations. Proc. Japan Acad. Ser. A Math., 49 (1973), 503-525.

[14] Henry, D., Geometric Theory of Semilinear Parabolic Equations. Springer-Verlag, Berlin, (1981).

[15] Henry, D., Semigroups. Course Notes. IME/USP, São Paulo, Brazil, (1981).

[16] Kaplan, S., On the growth of solutions of quasilinear parabolic equations. Comm. Pure Appl. Math., 16 (1963), 305-333.

[17] Lacey, A. A., Mathematical analysis of thermal runaway for spatially inhomogeneous reactions. SIAM J. APPL. MATH., 43 (1983), 1350-1366.

[18] Levine, H. A., Some nonexistence and instability theorems of formally parabolic equations of the form $P u_{t}=-A u+F(u)$. Arch. Rational Mech. Anal., 51 (1973), 371-386.

[19] Pazy, A., Semigroups of Linear Operators and Applications to Partial Differential Equations. Springer-Verlag, New York, (1963).

[20] Souplet, P., Weissler, F.B., Self-similar subsolutions and blow-up for nonlinear parabolic equations. J. Math. Anal. Appl., 212 (1997), 60-74.

[21] Walter, W., Nonlinear parabolic differential equations and inequalities. Current developments in partial differential equations, Temuco, (1999). Discrete and Continuous Dynamical Systems, 8(2) (2002), 451-468.

[22] Weissler, F. B., Existence and nonexistence of global solutions for a semilinear heat equation. Israel J. Math., 38 (1981), 29-40.

[23] Weissler, F. B., Single point blow-up of semilinear initial value problems. J. Differential Equations, 55 (1984), 204-224.

[24] Weissler, F. B., Local existence e nonexistence for semilinear parabolic equations in $L^{p}$. Indiana Univ. Math. J., 29 (1980), 79-102.

[25] Weissler, F. B., $L^{p}$-energy and blow-up for semilinear heat equation. Proc. Symp. Pure Math, 45 (parte 2) (1986), 545-552. 


\section{Índice remissivo}

Adjunto, 14

Anulador, 62

Aplicação dualidade, 12

Bordo parabólico, 77, 123

Conjunto total, 62

Desigualdade de Gronwall, 67

Espectro, 6

Função admissível, 126

Gerador infinitesimal, 6

Grupo de operadores, 25

Identidade do resolvente, 19

Imagem numérica, 14

Operador

auto-adjunto, 14

dissipativo, 12

fechável, 15

parte, 23

positivo, 42

setorial, 30

simétrico, 14

solução, 116

uniformente parabólico, 123

unitário, 25

Ponto de blow-up, 79
Potência fracionária, 39, 47

Pseudo-resolvente, 19

Resolvente, 6

Semigrupo, 5

analítico, 30

de contrações, 12

fortemente contínuo, 5

fortemente contínuo de contrações , 12

Solução

forte, 61,65

fraca, 61,65

Teorema

de Hille-Yosida, 9

de Hille-Yosida Forma Geral, 11

de Lumer-Phillips, 13

de Stone, 26

O Segundo Limite Fundamental, 17

Princípio do Máximo Forte, 125

Princípio do Máximo Fraco, 123, 124

Princípio do Mínimo - outra versão, 126

Princípio do Mínimo Forte - outra versão, 127 